FHWA/IN/JTRP-2005/2

Final Report

EFFECTS OF GEOMETRIC CHARACTERISTICS OF RURAL TWO-LANE ROADS ON SAFETY

Samuel Labi

June 2006 
FINAL REPORT

FHWA/IN/JTRP-2005/2

\title{
EFFECTS OF GEOMETRIC CHARACTERISTICS OF RURAL TWO-LANE ROADS ON SAFETY
}

\author{
By \\ Samuel Labi \\ Assistant Professor \\ School of Civil Engineering \\ and \\ Center for the Advancement of Transportation Safety (CATS) \\ Joint Transportation Research Program \\ Project No. C-36-59II \\ File No.: 8-5-35 \\ SPR-2664 \\ Prepared in Cooperation with the \\ Indiana Department of Transportation and \\ The U.S. Department of Transportation \\ Federal Highway Administration
}

The contents of this report reflect the views of the authors who are responsible for the facts and the accuracy of the data presented herein. The contents do not necessarily reflect the official views of the Federal Highway Administration and the Indiana Department of Transportation. The report does not constitute a standard, a specification, or a regulation.

Purdue University

West Lafayette, Indiana, 47907

June 2006 
TECHNICAL REPORT STANDARD TITLE PAGE

\begin{tabular}{|c|c|c|}
\hline $\begin{array}{l}\text { 1. Report No. } \\
\text { FHWA/IN/JTRP-2005/2 }\end{array}$ & 2. Government Accession No. & 3. Recipient's Catalog No. \\
\hline \multirow{2}{*}{\multicolumn{2}{|c|}{$\begin{array}{l}\text { 4. Title and Subtitle } \\
\text { EFFECTS OF GEOMETRIC CHARACTERISTICS OF RURAL } \\
\text { TWO-LANE ROADS ON SAFETY }\end{array}$}} & $\begin{array}{l}\text { 5. } \quad \text { Report Date } \\
\text { June } 2006\end{array}$ \\
\hline & & 6. Performing Organization Code \\
\hline \multicolumn{2}{|l|}{$\begin{array}{l}\text { 7. Author(s) } \\
\text { Samuel Labi }\end{array}$} & $\begin{array}{l}\text { 8. Performing Organization Report No. } \\
\text { FHWA/IN/JTRP-2005/2 }\end{array}$ \\
\hline \multirow{2}{*}{\multicolumn{2}{|c|}{$\begin{array}{l}\text { 9. Performing Organization Name and Address } \\
\text { Joint Transportation Research Program } \\
\text { 1284 Civil Engineering Building, } 550 \text { Stadium Mall Dr., Purdue University, } \\
\text { West Lafayette, IN 47907-1284 }\end{array}$}} & 10. Work Unit No. \\
\hline & & $\begin{array}{l}\text { 11. Contract or Grant No. } \\
\text { SPR-2664 }\end{array}$ \\
\hline \multirow{2}{*}{\multicolumn{2}{|c|}{$\begin{array}{l}\text { 12. Sponsoring Agency Name and Address } \\
\text { Indiana Department of Transportation, State Office Bldg, } 100 \text { N Senate Ave., } \\
\text { Indianapolis, IN } 46204\end{array}$}} & $\begin{array}{l}\text { 13. Type of Report and Period Covered } \\
\text { Final Report }\end{array}$ \\
\hline & & 14. Sponsoring Agency Code \\
\hline
\end{tabular}

\section{Abstract}

The study investigated the safety effects of geometric and other roadway characteristics of rural two-lane roads by collecting and analyzing data and developing crash prediction models, crash reduction factors (CRFs), and cost-effectiveness ratios (CERs). On the basis of these results, and the existing inventory of roads and their geometric deficiencies, physical and monetary needs assessments were carried out under alternative budgeting and crash costing scenarios for the rural two-lane state highway network. It was found that influential crash factors at such roads include section length, traffic volume, lane width, shoulder width, pavement condition, average horizontal curve radius, and grades of vertical slopes. For county roads, additional influential variables are shoulder type, driveway density, and the presence of speed limit posting. The percentage reduction in crashes due to the various safety countermeasures was found to vary by crash severity type, highway class, level of deficiency, and the extent to which the deficiency is addressed. CRFs were developed for lane and shoulder widening, minor realignment and earthworks that increase horizontal curve radius or to decrease in vertical curve slope, pavement friction enhancement (for rural major collectors and minor arterials) and pavement condition improvement (for rural principal arterials). CERs were developed for each countermeasure, crash type and road functional class. CERs are useful for safety project evaluation particularly when INDOT/LTAP seeks to compare the safety benefit per dollar across mutually exclusive safety countermeasures at a specific problem area, or when a specific area suffers from multiple safety problem types and can therefore receive many different countermeasures only a few of which can be funded. For rural two-lane state highway sections that are deficient-but-not-necessarily-hazardous and both-hazardous-and-deficient sections, it was found that $\$ 530$ million and $\$ 55$ million, respectively, would be needed to address the engineering safety needs. Given an annual expenditure of $\$ 1 \mathrm{M}$ over a 5-year period, approximately 170 crashes (translating to $\$ 8-15$ million, depending on crash costing method) could be saved. If the annual expenditure is increased to $\$ 2 \mathrm{M}$ over the same period, approximately 240 crashes (translating to \$12-26 million) could be saved. The study results also suggest that there seems to exist a ceiling of effectiveness for engineering safety countermeasures, and therefore non-engineering countermeasures such as safety education and enforcement are also vital to further reduce crashes related to drivers, vehicles, and the driving environment. Implementation of the study would involve application of the study products for safety management at both state and local levels to make cost-effective decisions that will reduce highway crashes and thereby accrue the secondary and indirect benefits of increased mobility, enhanced geriatric driving, reduced tort liability, and enhanced economic productivity.

\section{Key Words}

Fatal Crash, Serious Injury Crash, Property Damage Crash, Rural Two-lane Roads, Crash Reduction Factors, Crash Prediction, Safety Needs Assessment.

\section{Distribution Statement}

No restrictions. This document is available to the public through the National Technical Information Service, Springfield, VA 22161

\begin{tabular}{|c|c|c|c|}
\hline $\begin{array}{c}\text { 19. Security Classif. (of this report) } \\
\text { Unclassified }\end{array}$ & 20. Security Classif. (of this page) & 21. No. of Pages & 22. Price \\
\hline
\end{tabular}




\section{ACKNOWLEDGMENTS}

The author hereby acknowledges the contributions of Messrs. John Nagle of INDOT Safety Management Unit, John Habermann of LTAP, Rick Drumm of FHWA Indiana Office, Mr. John Evans of the Indiana State Police, and Dr. Shuo Li of INDOT Research Division. These gentlemen served on the study advisory committee and made valuable contributions at various stages of the project. The input from Mr. Godfrey Lamptey was pivotal as he carried out the task of merging data from various sources to develop a single database that could be used for the analysis. The help of Mr. John Ragan and Ms. Sravanthi Konduri in getting the study started is acknowledged. Ms. Konduri also helped carry out the questionnaire surveys of local police and initiated the modeling efforts. The efforts of Mr. Robert Zahnke, Mr. Jose Thomaz, and Ms.

Jennifer Howells, in providing overall administrative support for the study, are recognized. Also, several CATS personnel played vital roles on acquisition and formatting of crash data from the Indiana Crash Database and Accident Information System. Furthermore, the assistance of Ms. Abisola Kamson in field collection of county road inventory data is appreciated. Ms. Cindy Corddington of Tippecanoe County also provided valuable data on county road crashes. I am thankful for the efforts of Mr. Thomas Martin of LTAP for assisting in the acquisition of documented county road data, and Ms. Karen Hatke, the JTRP coordinator, for the help she consistently gave us throughout the course of this project. Finally, the overall support provided by Mr. Barry Partridge, Chief of INDOT’s Research Division is very much appreciated. 
TABLE OF CONTENTS

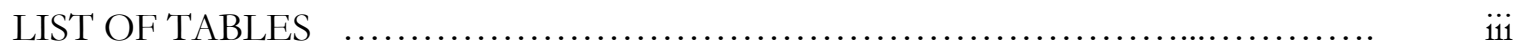

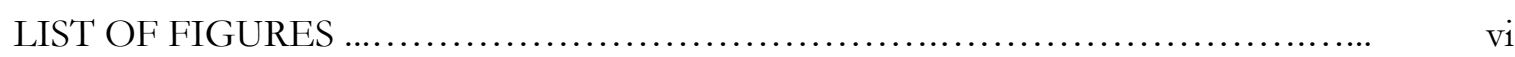

GLOSSARY OF ACRONYMS AND TERMS ................................ xiv

\section{CHAPTER 1 INTRODUCTION}

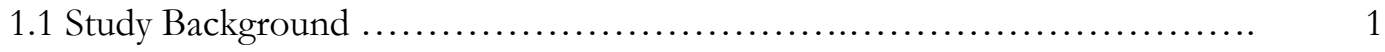

1.2 Problem Statement .............................................. 7

1.3 Objectives of the Present Study .................................... 12

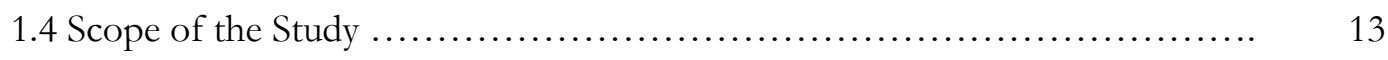

1.5 Overview of the Study Approach................................... 14

CHAPTER 2 RESULTS OF THE QUESTIONNAIRE SURVEY $\ldots \ldots \ldots \ldots \ldots \ldots \ldots \ldots \ldots \ldots$

\section{CHAPTER 3 LITERATURE REVIEW}

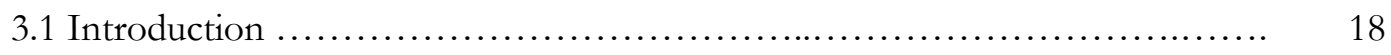

3.2 Crash Predicting Modeling........................................ 18

3.3 Alternative Methods for Identifying High Crash Locations ................. 23

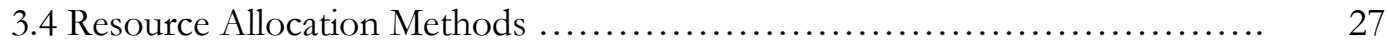

3.5 Existing Safety Analysis Software .................................. 28

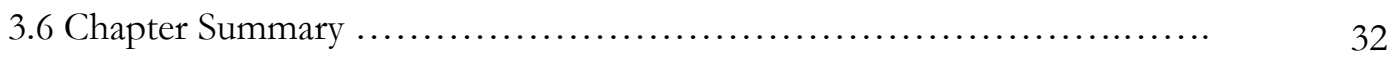

\section{CHAPTER 4 ANALYTICAL FRAMEWORK}

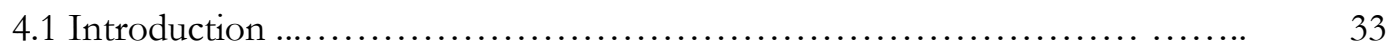

4.2 Development of Crash Prediction Models and Crash Reduction Factors..... 33

4.3 Framework for Needs Assessment for Indiana’s Rural Two-Lane Highways 45

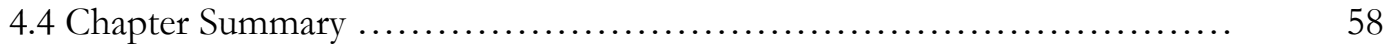


Page

\section{CHAPTER 5 DATA COLLECTION AND COLLATION}

5.1 Data Sources for Rural Two-lane State Roads .......................... 59

5.2 Data Sources for Rural Two-lane County Roads ........................ 65

\section{CHAPTER 6 DESCRIPTION AND DISTRIBUTION OF EXPLANATORY} CRASH FACTORS

6.1 Carriageway Factors.............................................. 70

6.2 Sectional and Alignment Factors .................................. 90

6.3 Shoulder Factors .................................................. 108

6.4 Roadside Environment ......................................... 122

6.5 Traffic Control Devices ................................................. 138

\section{CHAPTER 7 CRASH PREDICTION MODELS, CRASH REDUCTION} FACTORS, AND COST-EFFECTIVENESS RATIOS

7.1 Crash Prediction Models.......................................... 140

7.2 Development of Crash Reduction Factors ........................... 144

7.3 Estimation of Costs of Safety Countermeasures ....................... 159

7.4 Estimation of Cost-effectiveness of Safety Countermeasures ............... 160

\section{CHAPTER 8 NETWORK-LEVEL SAFETY NEEDS ASSESSMENTS}

8.1 Introduction.................................................. 173

8.2 Current INDOT Design Standards for Rural Two-lane State Highways ...... 173

8.3 Needs Assessment for Indiana’s Rural Two-lane State Highways ............. 174

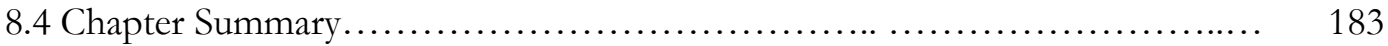

CHAPTER 9 SUMMARY AND CONCLUSIONS............................... 184

9.1 Factors Affecting Crash Occurrence ................................. 185

9.2 Crash Reduction Factors ........................................ 187

9.3 Cost-Effectiveness of Safety Enhancements ......................... 188

9.4 Safety Needs Assessments ........................................ 192

9.5 Concluding Remarks ......................................... 193

9.6 Implementation Issues ........................................ 196

9.7 Future Research ................................................ 197 


\section{APPENDICES}

APPENDIC A1: Distribution of Public Road Mileage by Area Class and

APPENDIX A2: Distribution of Public Road Mileage by Functional Class.........

APPENDIX A4: Distribution of Local Road System Road Mileage by Functional

APPENDIX A5: Distribution of Public Road VMT by Functional Class........

APPENDIX B: Newspaper Clipping on Rural Road Safety ............

APPENDIX C: Questionnaire Survey Solicitation Letter.............

APPENDIX D: Crash Prediction Modeling Results....

APPENDIX E: Priority Listing of Safety Projects 


\section{LIST OF TABLES}

Table

Page

Table 1-1: $\quad$ State Highway Inventory Size Distribution by Road Class . ................. 2

Table 3-1: $\quad$ Survey Results - Crash Prone Locations ........................... 16

Table 4-1: $\quad$ Categories of Models Developed in this Study.......................... 45

Table 4-2: $\quad$ Default Safety Improvement Projects in Indiana’s SMS Software Package... 52

Table 4-3: $\quad$ Unit Highway Crash Cost Values (2000 dollars) $\ldots \ldots \ldots \ldots \ldots \ldots \ldots \ldots \ldots \ldots \ldots \ldots \ldots$

Table 5-1: $\quad$ Variables in the Overall Modeling Dataset............................ 63

Table 6-1: $\quad$ Effectiveness of Lane Widening [Zegeer, 1991] ....................... 71

Table 6-2: $\quad$ Correlation Matrix for Lane Widths and Crashes....................... 78

Table 6-3: $\quad$ Correlation Matrix for Pavement Surface Friction and Crashes............. 79

Table 6-4: $\quad$ Correlation Matrix for Surface Condition and Crashes..................... 84

Table 6-5: $\quad$ Effectiveness of Designs for Passing Opportunities [Harwood, 1989]....... 86

Table 6-6: $\quad$ Accident Reduction Factors for Bridge Widening $\ldots \ldots \ldots \ldots \ldots \ldots \ldots \ldots \ldots . . \quad 88$

Table 6-7: $\quad$ Effectiveness of Curve Widening..................................... 94

Table 6-8: $\quad$ Effectiveness of Superelevation at Horizontal Curves.................... 95

Table 6-9: $\quad$ Effectiveness of Increasing Recovery Distance at Horizontal Curves ...... 97

Table 6-10: Accident Reduction Factors for Flattening Side-slopes at Curves.......... 97

Table 6-11: $\quad$ Correlation Matrix for Horizontal Curves and Crashes................... 98

Table 6-12: $\quad$ Crash Frequency and Severity by Vertical Alignment Feature .............. 102

Table 6-13: $\quad$ Accident Modification Factors for Grade Reduction .................... 103

Table 6-14: $\quad$ Correlation Matrix for Vertical Curves and Crashes ..................... 105

Table 6-15: $\quad$ Effectiveness of Shoulder Type and Widening [Zegeer, 1987]............... 109

Table 6-16: $\quad$ Accident Modification Factors for Various Shoulder Widths ............... 110

Table 6-17: Accident Modification Factors for Various Shoulder Types............... 110

Table 6-18: Correlation between Shoulder Type and Crashes..................... 120

Table: 6-19: Rumble Strips - States of Practice.............................. 121

Table 6-20: Accident Reduction Factors due to Increasing Roadside Recovery Distance 123

Table 6-21: $\quad$ Accident Reduction Factors for Increasing Roadside Recovery Distance ... 126

Table 6-22: $\quad$ Effectiveness of Obstacle-Removal Treatments ........................ 129 
Table

Page

Table 6-23: $\quad$ Reduction in Utility Pole Crashes due to Pole Relocation...................

Table 7-1: $\quad$ Crash Reduction Factors for Safety Improvements, Rural Major Collectors

Table 7-2: $\quad$ Crash Reduction Factors for Safety Improvements, Rural Minor Arterials

Table 7-3:

Crash Reduction Factors for Safety Improvements, Rural Principal Arterials

Table 7-4:

Crash Reduction Factors for County Roads

156

Table 7-5:

Safety Project Costs.

Table 8-1:

Indiana Design Standards for Rural Two-Lane State Highways

Table 8-2:

Summary of Identified Deficiencies on Rural Two Lane Roads.

175

Table 8-3:

Needs Assessment Scenarios...

176

Table 8-4:

Summary of Capital Requirement by Project Type (Scenario 1)

176

Table 8-5:

Summary of Capital Requirement by Project Type (Scenario 2).............

177

Table 8-6:

Summary of Capital Requirement by Project Type (Scenario 3).

178

Table 8-7:

Summary of Capital Requirement by Project Type (Scenario 4).

179

Table 8-8:

Summary of Capital Requirement by Project Type (Scenario 5).

180

Table 8-9:

Summary of Capital Requirement by Project Type (Scenario 6).

181

Table D-1:

Injury Crash Model for Rural Major Collectors..........................

209

Table D-2:

PDO Crash Model for Rural Major Collectors....

209

Table D-3:

Fatal+Injury Crash Model for Rural Major Collectors

210

Table D-4:

"Total Crashes" Model for Rural Major Collectors.

210

Table D-5:

Injury Crash Model for Rural Minor Arterials

211

Table D-6:

PDO Crash Model for Rural Minor Arterials....

211

Table D-7:

Fatal+Injury Crash Model for Rural Minor Arterials.

212

Table D-8:

"Total Crashes" Model for Rural Minor Arterials.

212

Table D-9:

Injury Crash Model for Rural Principal Arterials....

213

Table D-10:

PDO Crash Model for Rural Principal Arterials.

213

Table D-11:

Fatal+Injury Crash Model for Rural Principal Arterials.

214

Table D-12:

"Total Crashes" Model for Rural Principal Arterials.

214

Model for County Road Crashes Excluding Shoulder Width Variable........ 215

Table D-14:

Model for County Road Crashes Including Shoulder Width Variable.

215

Table E-1

Priority Ranking of Selected Safety Improvement Project, Scenario 2........ 


\section{LIST OF FIGURES}

Figure

Page

Figure 1-1: $\quad$ Distribution of State Highway System Size by Road Class, Year 2000 .............. 1

Figure 1-2: $\quad$ Safety Performance Trends for Indiana’s State Highway Network................... 3

Figure 1-3: $\quad$ Distribution of Total Crash Count by Road Class...............................

Figure 1-4: $\quad$ Distribution of Total Crash Density (Crashes per mile) by Road Class................ 4

Figure 1-5: $\quad$ Distribution of Total Crash Rate (Crashes per vehicle-mile) by Road Class ........... 4

Figure 1-6: Distribution of Annual Average Crash Frequency by Roadway Type and Crash Severity................................................................... 5

Figure 1-7: $\quad$ Roadway Cross-sectional Elements...................................... 7

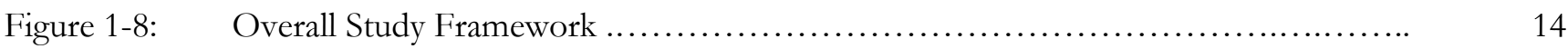

Figure 3-1: $\quad$ Modules and Tasks Addressed by the Indiana Safety Management System Software 31

Figure 4-1: $\quad$ Framework for Development of Crash Models .............................. 34

Figure 4-2: Sequence of Tasks for Identification of Countermeasures....................... 51

Figure 5-1: Components of the Comprehensive Road Safety Database.......................... 59

Figure 5-2: Concepts of Algorithm Used for Linking Data from the Various Datasets ........... 62

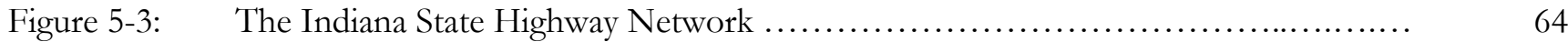

Figure 6-1: $\quad$ Explanatory Engineering Factors Affecting Crashes at Rural Two-lane Roads ........ $\quad 69$

Figure 6-2: $\quad$ Relationship between Lane Width and Crashes................................ 72

Figure 6-3: $\quad$ Relationship between Combined Width and Crashes............................. 73

Figure 6-4: Distribution of Lane Widths at Rural Two-lane Highways in Indiana, by Functional 75

Figure 6-5: $\quad$ Distribution of Lane Widths at Rural Two-lane Highways in Indiana, by Region ..... 76

Figure 6-6: $\quad$ Variation of Crash Rates with Lane Width by Crash Severity and Functional Class ... 77

Figure 6-7: Distribution of Average Pavement Friction at Rural Two-lane Highways in Indiana, by Functional Class ......................................................... $\quad 80$ 
Figure 6-8: Distribution of Average Pavement Friction at Rural Two-lane Highways in Indiana, by

Figure 6-9: Variation of Crash Rates with Pavement Friction by Crash Severity and Functional Class

Figure 6-10: $\quad$ Typical Treatments for Addressing Passing Operations on Rural 2-Lane Roads ......

Figure 6-11: $\quad$ The Concept of "Relative" Bridge Width [Turner, 1984] …................... 87

Figure 6-12: $\quad$ Relationship between Crash Rate and "Relative" Bridge Width...................... 88

Figure 6-13: $\quad$ Relationship between Mean Crash Rate and Mean Degree of Curvature................ 93

Figure 6-14: Distribution of Horizontal Curve Density at Rural Two-Lane State Highways, by Functional Class...

Figure 6-15: Distribution of Horizontal Curve Density at Rural Two-Lane State Highways, by

Figure 6-16: Variation of Crash Rates with Horizontal Curve Density by Functional Class........... 100

Figure 6-17: Variation of Crash Rates with Vertical Curve Density by Functional Class............... 106

Figure 6-18: Relationship between Lane Width and Crashes............................. 111

Figure 6-19: Shoulder Width at Distribution at Rural Two-lane State Highways in Indiana, by 113

Figure 6-20: Shoulder Width Distribution at Rural Two-lane State Highways in Indiana, by Region

Figure 6-21: Variation of Crash Rates with Shoulder Width by Crash Severity and Functional Class

Figure 6-22: Distribution of Shoulder Type on Rural Two-lane Highways in Indiana, by Functional

Figure 6-23: Distribution of Shoulder Type on Rural Two-lane Highways in Indiana, by Region ...

Figure 6-24: Variation of Crash Rates with Shoulder Type by Crash Severity and Functional Class

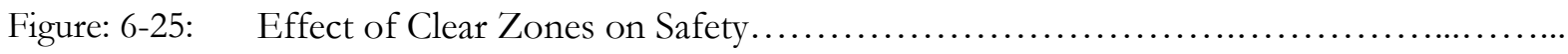

Figure 6-26: Relationship between Side-slopes and Single-vehicle Crashes

Figure 6-27: Illustration of Side-slope Design at Fill Section.

Figure 6-28: Relationship between Frequency of Utility Pole Crashes and Pole Offset/Density ....

Figure 6-29: Nomograph for Predicting Utility Pole Crash Frequency $\ldots \ldots \ldots \ldots \ldots \ldots \ldots \ldots \ldots \ldots$

Figure 7-1: Crash Reduction Factors by Improvement Type and Crash Type, Rural Major

Figure 7-2: Crash Reduction Factors by Improvement Type and Crash Type, Rural Minor 
Figure 7-3: Crash Reduction Factors by Improvement Type and Crash Type, Rural Principal

Figure 7-4: Cost-effectiveness by Improvement Type and Crash Type, Rural Major Collectors ...

Figure 7-6: Cost-effectiveness by Improvement Type and Crash Type, Rural Principal Arterials... 168

Figure 7-7: $\quad$ Effect of Lane Widening at Typical Rural Two-Lane Sections...................... 171

Figure 7-8 Effect of Shoulder Widening at Typical Rural Two-Lane Sections ...................

Figure 8-1: $\quad$ Summary of Identified Deficiencies on Rural Two-Lane State Highways in Indiana ... 


\section{LIST OF PLATES}

Plate

Page

Plate 1-1: Many Rural Two-lane Roads in Indiana have Problems Associated with Road Width, Shoulder Type and Width, Pavement Marking, Roadside Hazards, and Other Deficiencies

Plate 5-1: County Road Data Collection by the Research Team, April 2003.......................... 66

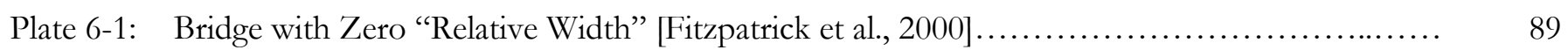

Plate 6-2: Superelevation and Pavement Material Stabilization May Help to Stabilize Vehicles Negotiating County Road Horizontal Curves

Plate 6-3: Inadequate Vertical Curve Sight Distance May Lead to High Crash Rates

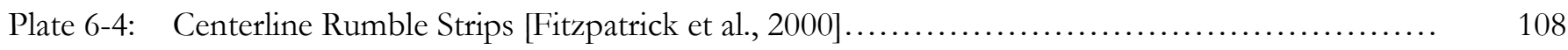

Plate 6-5: Shoulder Rumble Strips [Harwood et al., 2000]..................................... 120

Plate 6-6: Road Side Hazard Ratings: Conceptual Illustrations ................................ 134

Plate 6-7: More Frequent Driveways at County Road Sections May lead to Higher Crash Rates .......... 136

Plate 6-8: The White-tailed Deer is a Common Culprit (and Victim) of Crashes at Indiana's Rural Two- 137 Lane Roads ..............................

Plate 9-1: Few County Roads Have Adequate Geometrics and Pavement Markings ................... 195

Plate 9-2: Loss of Paved Surfaces of County Roads due to Inadequate Preservation Since Construction .. 


\section{GLOSSARY OF ACRONYMS AND TERMS}

AADT

AASHTO

CATS

$\mathrm{EB}$

FATINJ

FHWA

IIHS

IHSDM

INDOT

LSS

LTAP

RLW

NB

NCHRP

OD

PDO

PSI

RHR

ROR

SMS

TRB

VMT
Average Annual Daily Traffic

Association of American State Highway and Transportation Officials

Center for Advanced Transportation Safety

Empirical Bayesian Method for crash prediction

Fatal and Injury Crashes (also sometimes referred to as "casualty" crashes)

Federal Highway Administration

Insurance Institute for Highway Safety

Interactive Highway Safety Design Model

Indiana Department of Transportation

Least Sum of Squares method of statistical estimation

Local Technical Assistance Program

Right Shoulder Width

Negative Binomial (a generalized form of the Poisson distribution)

National Cooperative Highway Research Program

Opposite-Direction Crashes

Property Damage Only

Present Serviceability Index

Roadside Hazard Rating

Run-off-the-Road Crashes

Safety Management System

Transportation Research Board

Vehicle Miles of Travel
Crash Prediction Equations

Crash Patterns

Crash Severity

Pseudo-code
Same as Safety Performance Functions

Single vehicle (roll-over); Multi-vehicle (rear-end, head-on, etc.)

Fatal; Serious Injury; Property Damage Only (PDO)

A unique identifier code assigned by the Indiana Crash Database to road sections for purpose of crash location description. 


\section{CHAPTER 1 INTRODUCTION}

\subsection{STUDY BACKGROUND}

\subsubsection{General Safety Issues and Trends at Indiana's Road Network}

This section discusses the inventory and general safety performance trends at the state and local rural two-lane roads vis-à-vis the overall road network. Details of the distributions are provided in Appendix A.

\subsubsection{The State Highway Network}

System Size: The Indiana state highway network, which has over 11,000 miles of road and constitutes about $10 \%$ of the entire road network in the state, can be classified as follows: Rural Two-lane, Rural Multi-lane, Urban Two-lane, Urban Multi-lane, Rural Interstates and Urban Interstates. Figure 1-1 shows the mileage distribution of the state highway sections by road class.

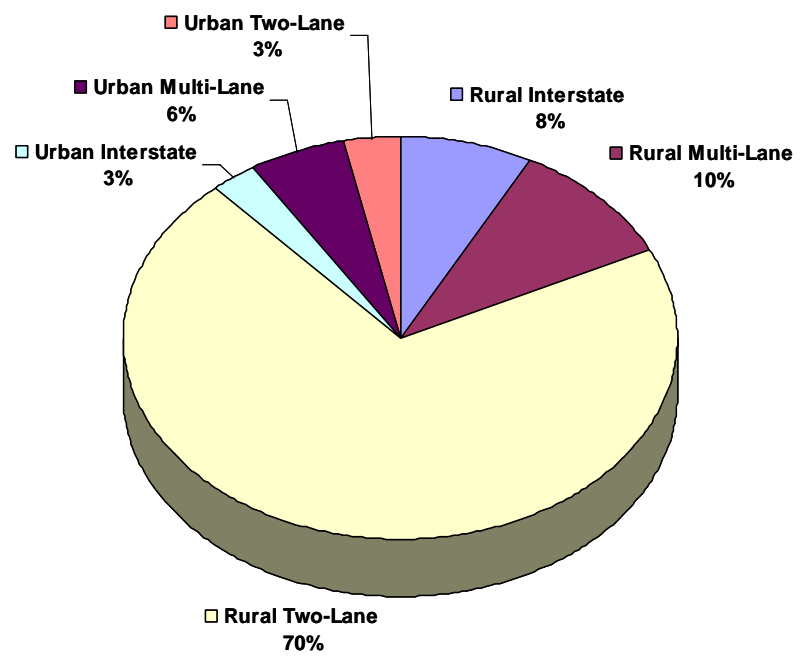

Figure 1-1: Distribution of State Highway System Size by Road Class, Year 2000

Rural roads with only two-lanes account for $70 \%$ of the network, constituting the largest single group on the state highway network (Figure 1-1). Table 1-1 shows the distribution by road class as of the year 2000. A review of recent inventory data (Year 2005) indicates that over the past 5 years, there have been very little changes in the indicated road inventory size and distribution. 
Therefore, the road class distribution as of the time of reporting is expected to be similar to that shown in Figure 1-1 and Table 1-1.

Table 1-1: State Highway Inventory Size Distribution by Road Class

\begin{tabular}{|c|c|c|c|c|}
\hline \multicolumn{2}{|r|}{ Road Class } & \multirow{2}{*}{$\begin{array}{c}\begin{array}{c}\text { Number of } \\
\text { Sections }^{1}\end{array} \\
172\end{array}$} & \multirow{2}{*}{$\begin{array}{c}\begin{array}{c}\text { Length } \\
\text { (miles) }\end{array} \\
871.61\end{array}$} & \multirow{2}{*}{$\begin{array}{c}\begin{array}{c}\text { Percentage } \\
\%\end{array} \\
7.91 \%\end{array}$} \\
\hline Rural Interstate & Rural Interstate & & & \\
\hline \multirow{3}{*}{ Rural Multi-Lane } & Rural Major Collector & 32 & 159.06 & $1.44 \%$ \\
\hline & Rural Minor Arterial & 34 & 168.67 & $1.53 \%$ \\
\hline & Rural Other Principal Arterial & 126 & 755.02 & $6.85 \%$ \\
\hline \multirow{5}{*}{ Rural Two-Lane } & Rural Local Roads ${ }^{3}$ & 9 & 16.57 & $0.15 \%$ \\
\hline & Rural Major Collector ${ }^{3}$ & 886 & 4687.66 & $42.53 \%$ \\
\hline & Rural Minor Arterial & 367 & 2052.73 & $18.62 \%$ \\
\hline & Rural Minor Collector & 5 & 16.85 & $0.15 \%$ \\
\hline & Rural Other Principal Arterial & 182 & 1013.53 & $9.20 \%$ \\
\hline Urban Interstate & Urban Interstate & 45 & 305.71 & $2.77 \%$ \\
\hline \multirow{2}{*}{ Urban Multi-Lane } & Urban Other Freeways and Expressway & 22 & 112.80 & $1.02 \%$ \\
\hline & Urban Other Principal Arterial & 86 & 506.97 & $4.60 \%$ \\
\hline \multirow{4}{*}{ Urban Two-Lane } & Urban Collector $^{3}$ & 2 & 1.46 & $0.01 \%$ \\
\hline & Urban Local Streets ${ }^{3}$ & 3 & 1.84 & $0.02 \%$ \\
\hline & Urban Other Arterial & 57 & 103.12 & $0.94 \%$ \\
\hline & Urban Other Principal Arterial & 74 & 249.01 & $2.26 \%$ \\
\hline & TOTAL & 2,102 & $11,022.61$ & $100 \%$ \\
\hline
\end{tabular}

1. A "Section" is defined as the stretch of road between two major intersections.

2. On the basis of the distribution of section lengths. 3. These refer to parts of the local system that are under INDOT jurisdiction. Source of data: Indiana Safety Management Database [INDOT, 2003]

Crash Occurrence: Average trends from 1997 to 2000 indicate that crashes on Indiana's state highway network account for about $25 \%$ of all crashes on the entire road network in the state $-75 \%$ occur at non state highways (local roads not under INDOT jurisdiction and county roads). Also, approximately $70 \%$ of all crashes on the state highway network occur at road sections while $30 \%$ occur at intersections. Within the period 1997-2000, the total number of crashes on the state highway network fluctuated slightly but stayed within the 60,000-65,000 range (Figure 1-2). The 
annual number of fatal crashes has generally hovered around 420, showing a slight increase from 1997 to 1999 and dropping considerably in Year 2000 to approximately 400. The number of injury crashes (approximately 15,000) showed a slight but consistent decrease while PDO crashes (approximately 47,000) dipped in 1998 but increased slightly thereafter.

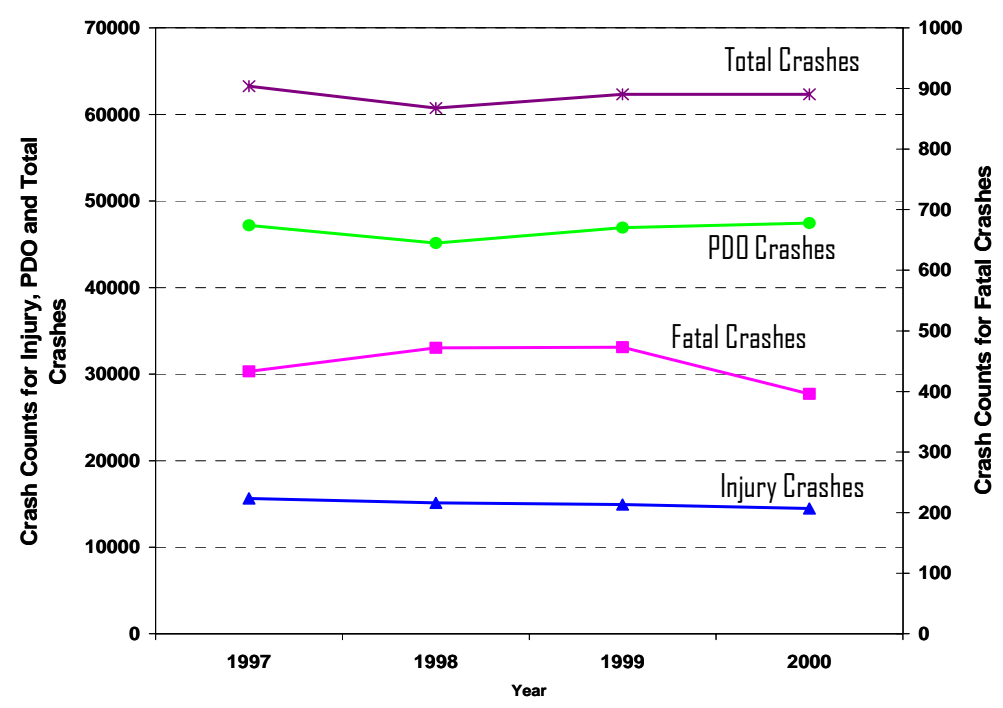

Figure 1-2: Overall Safety Performance Trends for Indiana’s State Highway Network

Figures 1-3 to 1-5 present comparative safety performance levels by state highway class over the 1997-2000 period. It is seen that rural two-lane roads had the highest total number of crashes while the urban two-lane roads had the least. This is obviously because rural two-lane roads account for $70 \%$ of the state highway network inventory while urban two-lane roads account for only $3 \%$.

On the basis of crash densities (number of crashes per mile), it is seen that rural two-lane roads had a better safety performance compared to their urban counterparts, obviously due to the sheer size of the rural two-lane road inventory vis-à-vis crash frequency at such roads. On the basis of crash rates (crashes per vehicle-miles traveled), rural two-lane roads had the poorest safety performance levels. This is obviously due to their relatively low traffic volume and consequently, high "crash frequency to traffic volume" ratio for such roads.

Overall, for most road classes, crash rates for all crash severity types generally declined over the 1997-2000 period obviously due to increased traffic volume, and also possibly because of increased enforcement and education, and engineering investments (implementation of physical safety projects). 


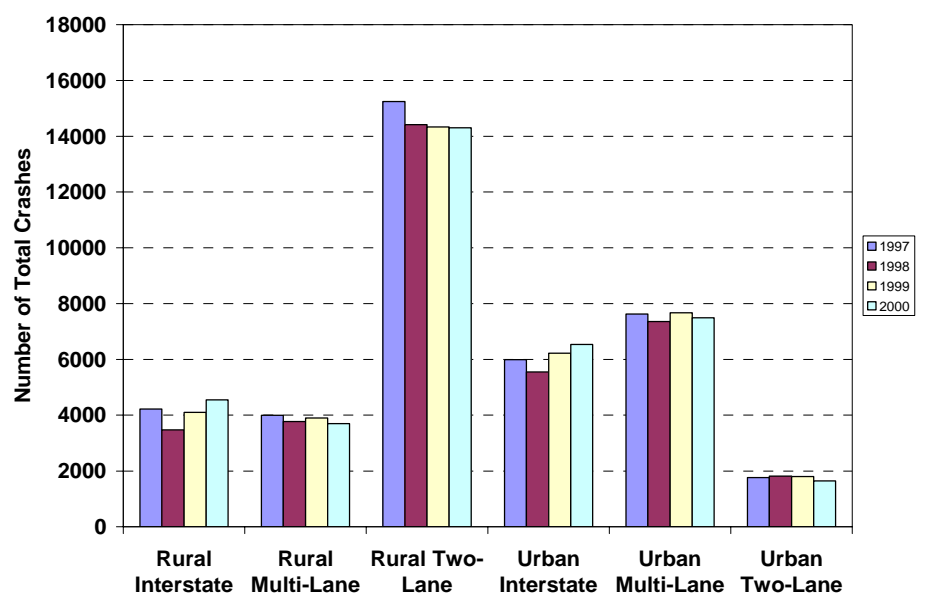

Figure 1-3: Distribution of Total Crash Count by Road Class

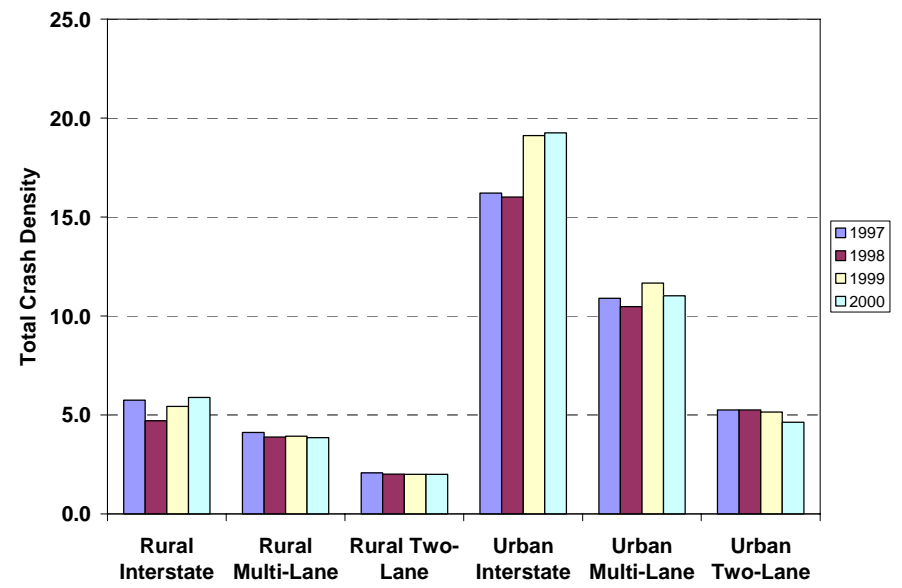

Figure 1-4: Distribution of Crash Density (Crashes per mile) by Road Class

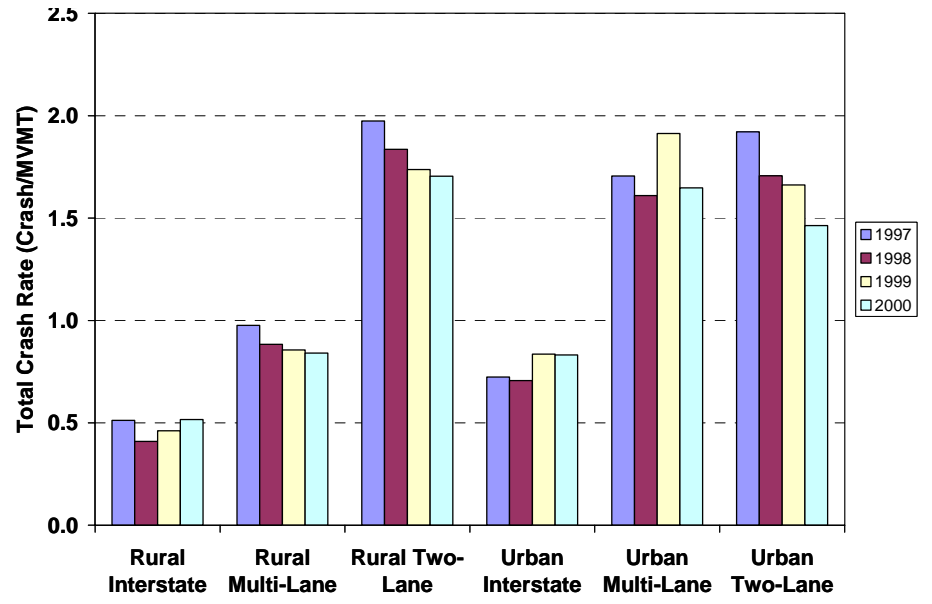

Figure 1-5: Distribution of Crash Rate (Crashes per vehicle-mile) by Road Class 


\subsubsection{The County Road Network}

Similar to the case for rural two-lane state highway roads, the safety situation for the county road system is indeed a source of great concern when viewed in comparison to other road classes. Figure 1-6 [CATS, 2003] shows the crash distribution by crash severity and roadway type including county roads. It is seen that of all highway classes, county roads have the highest number of fatal crashes, the second highest number of injury crashes, and the second highest number of PDO crashes. Given its vast expanse, the county road network is expected to have the least crash densities compared to other roads. As such, certain safety analysts may argue that the safety situation at county roads is not critical when compared to other roads. However, the crash statistics for the county road network are particularly disquieting when viewed against their relatively low traffic volumes. In fact, county roads have the highest crash rates expressed in terms of crashes per AADT.
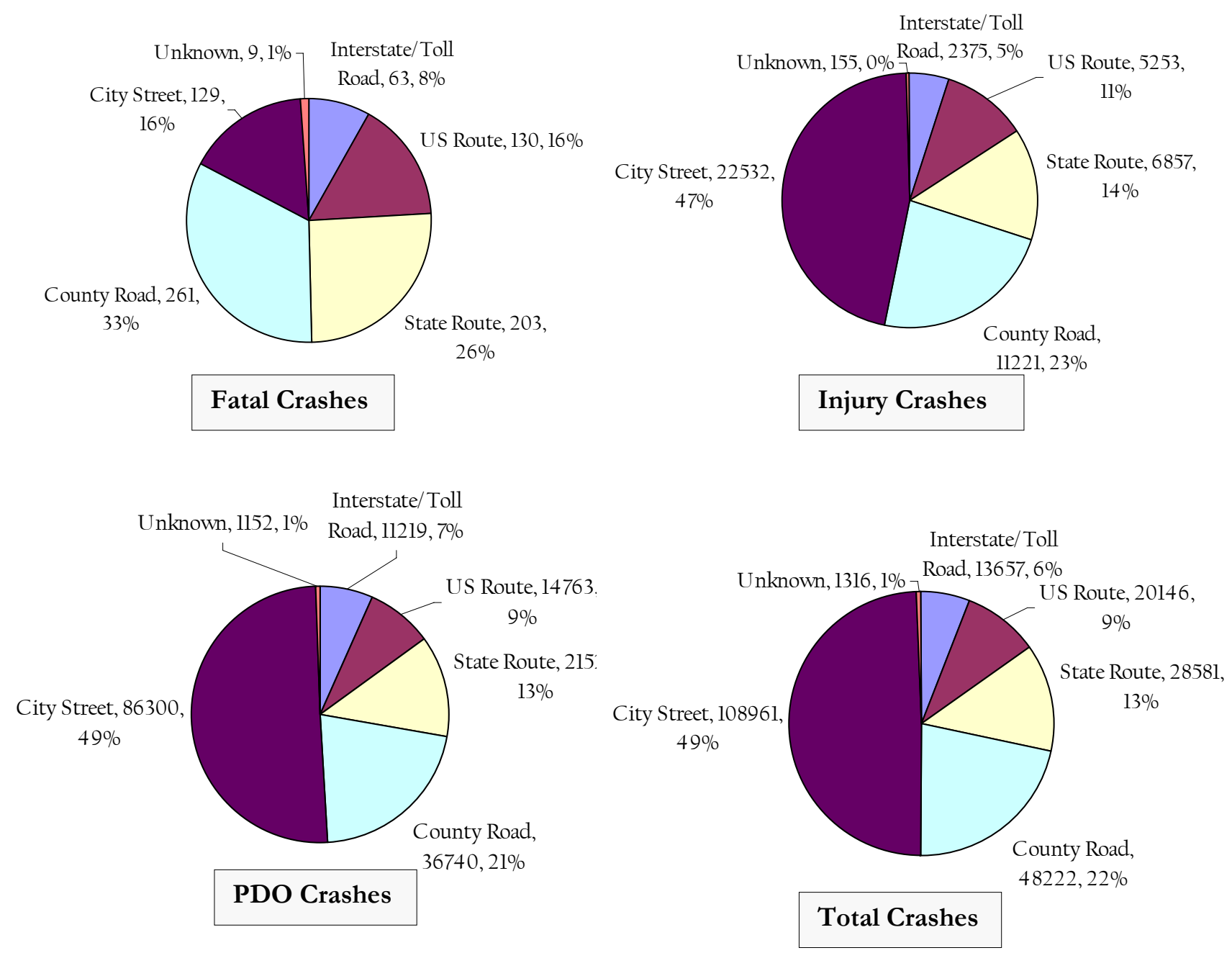

Figure 1-6: Distribution of Annual Average Crash Frequency by Roadway Type and Crash Severity 


\subsection{PROBLEM STATEMENT}

\subsubsection{Crash Experience at Rural Two-lane Roads -- The General Picture}

From the preceding section, it is clear that the vast majority of Indiana's road system consists of rural two-lane roads and that the traffic safety situation is more critical at such roads compared to other road classes. Even the local road system is largely dominated by county roads with only two lanes and in rural locations. As such, any effort geared at addressing the safety problems at rural two-lane roads is expected to accrue significant benefits to a large percentage of the state and local road network. The critical safety situation for rural two-lane roads is not unique to Indiana but is a nationwide problem (Appendix B).

Crash experience at rural two-lane roads is a culmination of several factors including highway geometry, driver characteristics, vehicle characteristics, and the environment. Crashes are typically caused by an interaction of more than one of these factors. For instance, an intoxicated person driving a defective vehicle at excessive speed on a geometrically deficient rural two-lane road is far more likely to experience a crash than a sober and alert driver of a roadworthy vehicle traveling at a reasonable speed on a geometrically adequate rural two-lane road.

With regard to geometric characteristics of rural two-lane roads, the current situation leaves much to be desired. In the United States, most of such roads have operational and safety deficiencies arising from inadequate road geometry, driver information deficiencies, lack of passing opportunities, and traffic conflicts due to driveways [McCree, 1988]. In fact, most rural two-lane roads are several decades old (since construction) and need pavement/shoulder replacement or repair, among other improvements [Zeeger at el., 1994]. Furthermore, most of such roads were designed and built to standards that have become outdated vis-à-vis current design policy. For example, most rural twolane state highways in Indiana have lane widths of $10 \mathrm{ft}$ or less; $40 \%$ have shoulder widths of $4 \mathrm{ft}$ or less and 10\% have no shoulder [INDOT, 2003]. A sample of county roads in Indiana showed that over $90 \%$ have little or no discernable shoulder (Plate 1-1). These statistics stand in stark contrast with current rural two-lane road design values in INDOT Design Manual (which calls for 11-12 ft lane widths and 4-6 ft shoulder widths) and in AASHTO's Green Book - Policy on the Geometric Design of Highways and Streets - which advocates 11-12 ft wide lanes, regardless of terrain, at rural two-lane roads.

The general relationships between crash rates and engineering factors (highway geometric characteristics) have been well established in the literature. Most studies that investigated this

relationship set out by first providing unequivocal definitions of road geometric features. For 
example, a road shoulder is defined as the section of a roadway that lies immediately adjacent to the carriageway (Figure 1-7). Paved roads typically have lines marking the delineation between the carriageway and the shoulder. For unpaved roads, the shoulder refers to the extra width of roadway beyond the gravel surface up to the inner edge of the side ditch and is typically covered by grass. The shoulder serves as a recovery zone where errant vehicles may be brought under control and also provides a zone where a driver may seek refuge to avoid being struck by another vehicle intruding into its path. Shoulders also lend lateral structural stability to the carriageway pavement.

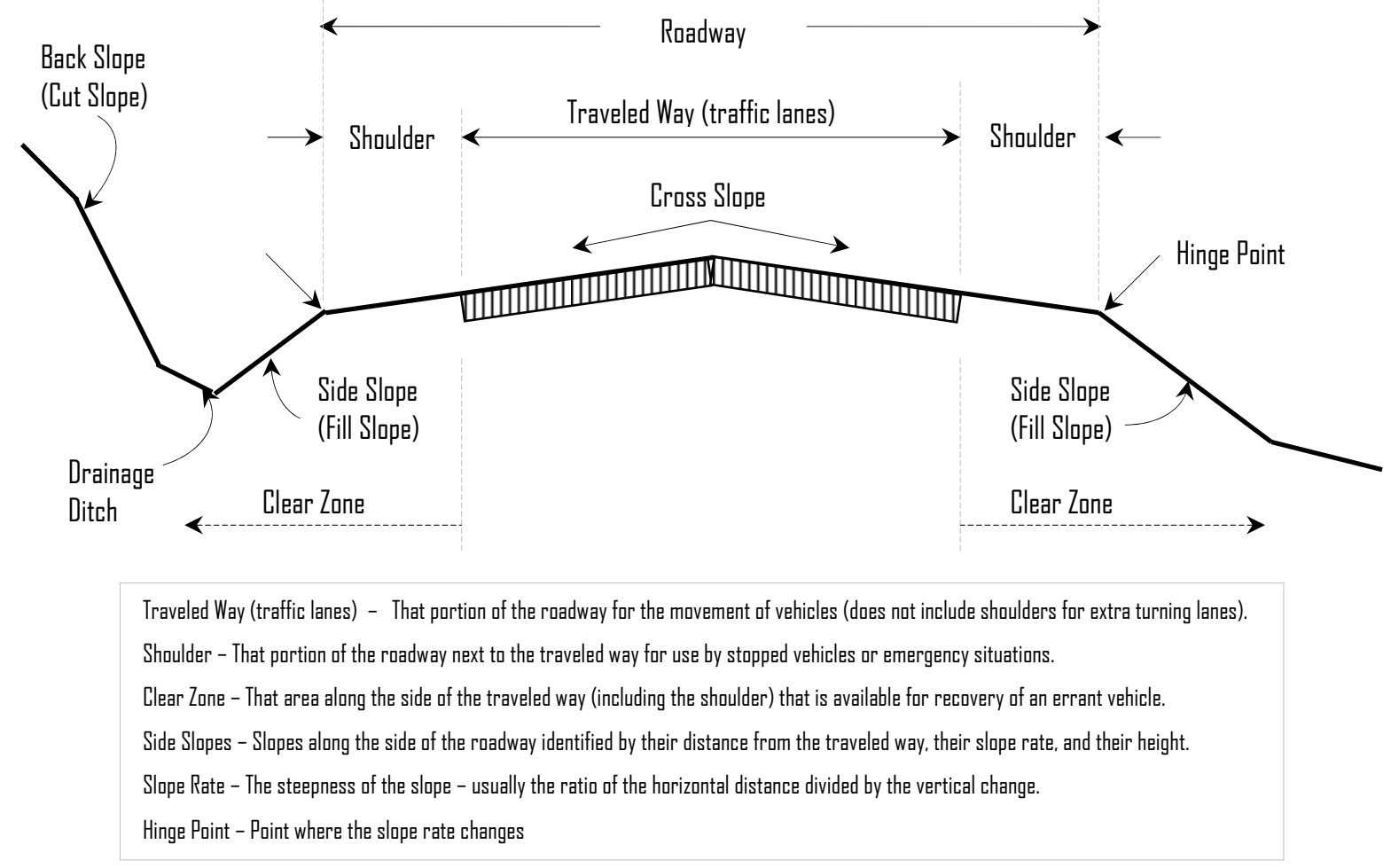

Figure 1-7: Roadway Cross Sectional Elements [FHWA. 1986]

Is it possible to reconstruct (or improve) Indiana's entire rural two-lane state highway and county road systems to design standards consistent with INDOT or AASHTO requirements? Obviously not, for the sheer volume of such undertaking is clearly not within the financial capabilities of state and local governments. On the other hand, allowing the roads to operate under their current geometric deficiencies is also not an attractive option: increased in crashes, vehicle operating costs, delays and inconveniences arising from the existing operational deficiencies often translate into driver frustration and lost time which may in turn exacerbate safety problems that 
already exist due to inadequate geometry. Obviously, it would be desirable to focus attention on areas that are identified as needing urgent intervention. In this regard, INDOT and local highway agencies in Indiana are faced with the task of evaluating the costs and effectiveness of specific safety improvements, identifying locations that urgently require some safety intervention, and developing a long-term program that prioritizes and selects rural two-lane road sections for needed safety improvements.

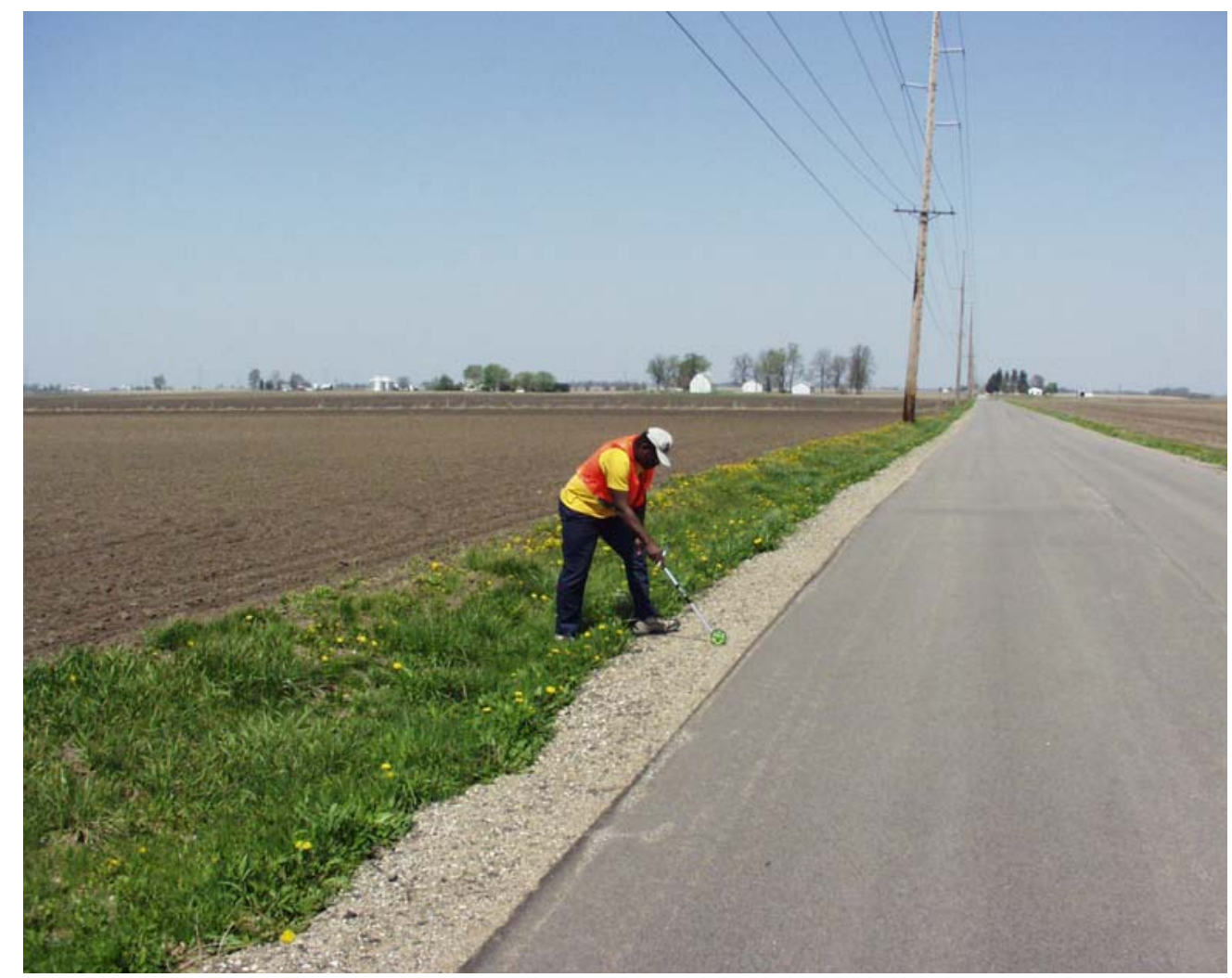

Plate 1-1: Many Rural Two-lane Roads in Indiana Have Problems Associated with Road Width, Shoulder Type and Width, Pavement Marking, Roadside Hazards, and Other Deficiencies

\subsubsection{Crash Experience at Rural Two-lane Roads - Specific Issues}

The need to investigate the crash experience/road geometric relationships is underscored by recent trends in the highway administration environment such as the need for effective management and operational accountability of highway assets and issues of funding and tort liability. Of the various road classes on the state roadway network, the rural two-lane state highway and county road systems are obviously most vulnerable to tort, due to their relatively poor geometric standards. Impacts of the loss of states' sovereign immunity in the 1970's are starting to be felt in the current era as many 
states including Indiana are now beginning to experience increased frequency of tort suits and growing expenditure to investigate and settle highway-related tort liability cases. In recent years, such expenditure has reached the order of millions of dollars in taxpayers money. At the present time, most tort liability cases in Indiana result from relatively benign problems such as mailbox damage during snow removal, pothole damage to vehicles, paint damage to passing vehicles during pavement marking operations, and damage due to mowing operations [Giraud et al., 2003]. However, if current trends continue, excessive frequency and levels of tort liability cases associated with the use of the highway system, particularly the rural two-lane system, could be expected in the future.

Is the state actually responsible for injury or death of road users due to real or perceived deficiencies on the state and local rural two-lane and other road systems? Yes and no. Design and maintenance decisions based on budgetary or other economic constraints are generally seen as discretionary in nature and consequently are generally immune from tort suits. However, as demonstrated in past cases, a transportation agency may argue that its failure to remedy a defective design is due to funding priorities but can be held liable if the agency presents no evidence on planning or ordering of priorities [Thomas, 2003]. In this regard, the development of a program that identifies problems on Indiana's rural two-lane highway system, develops safety needs assessments and establishes priority schedules for safety investment at such roads would be helpful for preemptive risk management. By providing evidence on planning and programming of investments, such programs will place the state in a better position to defend itself against claims related to highway engineering deficiencies.

Another vital issue is that of funding for improving safety at Indiana's rural two-lane roads. Current budgeting practice is characterized by allocation of most roadway funds for road planning, construction and preservation, with little or no funds left for sustained safety program implementation. Recognizing this need, the Intermodal Surface Transportation Efficiency Act (ISTEA) of 1991 required a specified minimum allocation of funding for safety projects from major highway programs such as the Surface Transportation Program (STP). Traditionally, roads under local jurisdiction receive significant portions of local funding from state and local taxes, not federal aid. As such, the ISTEA legislation, while of a generally encouraging nature, did not seem to be felt at the county level. Fortunately, the FHWA currently sets aside funds (Hazard Elimination and Safety Funds (HES)) for local agencies to support roadway safety activities. Practically any type of public surface transportation facility improvements including bicycle and pedestrian facilities may be implemented using HES funds provided the main purpose of the improvement is to eliminate traffic hazards or to substantially improve safety problems at the selected locations. It should be noted 
however, that any HES-financed local safety project should be located at roads that are classified as "local road" or "rural minor collector" or higher. For the state of Indiana, FHWA HES funds are approximately $\$ 6.9$ million annually, and can be carried over from year to year. There is also an HES Sanction Fund (approximately $\$ 12$ million per year). In addition to this, Indiana is required to transfer $3 \%$ of its highway construction funds to the HES Fund if the state has not enacted a Repeat Offender Intoxicated Driver (ROID) law. In order to have access to these funds, local agencies need to establish eligibility for all intended safety improvement projects using a process called the Safety Management Program. A Safety Management Program or System is defined as a systematic and consistent process of identifying and prioritizing safety needs, evaluating remedial actions and securing the resources to meet those needs, thereby protecting transportation networks as well as reducing the number and severity of collisions (Farouk et al., 1994; Haff, 1998).

A need therefore exists to investigate the relationships between crash rates and various geometric conditions (such as curve densities, lane width, and shoulder width) and shoulder type (paved vs. unpaved) at two-lane rural highways and county roads in the state of Indiana. On the basis of these relationships, it would be possible to determine the effect of improving such physical characteristics on highway safety. Then it would be possible to update crash reduction factors for (and also to evaluate the cost effectiveness of) such improvements and to prioritize safety investments for such roads. Furthermore, there is a need to determine the optimal schedule (and yearly priority listing) of safety improvements needed at the rural two-lane sections, the funding levels needed, and the impacts and cost-effectiveness in terms of decreased fatal, injury and PDO crashes, using safety management tools and techniques. This information will assist state decisionmakers in safety resource allocation, and also to evaluate the safety implications of alternative levels of specific safety investments on such highway segments. The application of the results to practice is expected to lead to more productive use of such highways, better allocation of resources of engineering-related safety investments, and more effective management of crash risks.

\subsection{STUDY OBJECTIVES}

In addressing the issues raised in the problem statement, the present study investigates the engineering factors of crash occurrence at Indiana's rural two-lane roads and subsequently strives to provide answers to the following questions:

1. Influential Factors: Which engineering factors influence crash rates on rural two-lane state highways and county roads in Indiana? Are these factors same or different from those found in past studies in Indiana or at other states? 
2. Extent of Influence: To what extent does each influential factor affect crashes on rural two-lane roads? Which factors are not significant, marginally significant, or very significant?

3. Possible Countermeasures: What are the feasible engineering treatments for addressing specific safety problems?

4. Efficacy of Countermeasures: What are the crash reduction factors (CRF) for each safety treatment type (i.e., countermeasure) such as shoulder widening, lane-widening, curve elimination, etc., for Indiana's rural two-lane roads. For example, how many lives can be expected to be saved for each $1 \mathrm{ft}$ increase in shoulder width?

5. Cost: What are the typical costs of lane widening, shoulder widening, shoulder paving and other safety treatments?

6. Cost-effectiveness of Countermeasures: What is the cost-effectiveness of each treatment in terms of crash reduction per dollar spent? For example, how many injury crashes can be avoided for every $\$ 1,000$ invested in lane widening, etc.?

7. Network level Safety Planning and Programming: Given an annual safety budget, what is the best mix of safety treatments that should be carried out (that is, what treatment, at which section, and in which year) such that the safety funds can yield the highest returns (crashes reduced) per dollar of spending? How sensitive is such optimal practice to major safety management input parameters such as budgetary limit and method of crash costing? How can INDOT or LTAP draw up priority lists for their hazardous locations?

8. Information (including Database) for Future Studies and Decision-support. In the course of addressing its primary objectives itemized above, the present study has a secondary objective - to develop a comprehensive integrated database for all rural two-lane segments on the state highway system and a sample of county roads. This study product could be expanded with updated data on an annual basis and used for future similar studies.

Information for comprehensive highway safety planning for rural two-lane roads garnered by the present study will help INDOT and LTAP to make decisions that will reduce highway crashes, and ultimately, to increase mobility, reduce tort liability, and enhance economic productivity. Given the ever-increasing usage of rural highways, even a marginal impact of such benefits can be significant in terms of the reduction of crash frequency and/or severity and othersecondary or indirect benefits. 


\subsection{SCOPE OF THE STUDY}

- Coverage: The present study is for rural two-lane roads in Indiana. Each of the 540 rural twolane state highway segments and a sample of 107 county road segment lengths ranging from approximately 0.19 to 0.97 miles, constituted the primary statistical unit for the analyses. Requisite data on crashes, geometric and traffic, were collected for each segment. For purposes of the study, "rural" and "two-lane roads" are defined as follows.

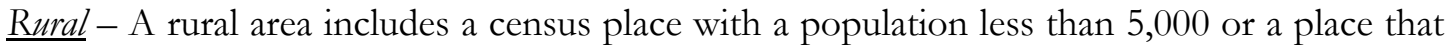
is not "urban". As a minimum, an urban area includes a census place with an urban population of 5,000 to 49,000 or a designated urban area with a population of 50,000 or more. "Rural" is defined as anything not Urban. Rural roads usually serve travel of primarily intra-county nature (FHWA, 2000).

Two-lane roads - For purposes of the present study, a two-lane road is simply defined as a roadway whose cross-section is characterized by a single lane for traffic in each direction (Figure 1-7). The lanes may be marked or unmarked. Also, the pavement is generally paved, but in a few cases (particularly at certain county roads), the road surface is either only stabilized, chip-sealed, or consists of compacted natural gravel or aggregates.

A "segment" is defined as a section of road between major intersections or where there is a significant change in geometric characteristics. Intersection crashes were excluded. Segments for the study were taken from the Indiana Safety Management database. County road segments were obtained from a database complied after a field survey by the Research Team in April 2003.

- Analysis Period: For the rural state highways, the study period ranges from 1997 to 2000.

- Road Functional Class: The present study focused only on rural two-lane roads. Four (4) functional classes were studied: Rural Collectors, Rural Minor Arterials, Rural Principal Arterials on the state highway system, and County Roads.

- Geo-climatic Region: The present study utilized data from pavement sections that were located at various locations in the state, without any geographical restriction.

- Crash Severity Types: These are fatal, injury, and property-damage-only crashes. 
- Crash Pattern Types: In specifying appropriate countermeasures at the network-level needs assessment stage of the study, the present study implicitly considered crash pattern types (such as roll-over, run-off-the-road, etc.). However, crash prediction models were developed for the crash severity types and not for crash pattern types.

- Geometric Characteristics: The study investigated specific characteristics such as lane width, shoulder width, horizontal curve density and radius, and vertical curve density and grade slopes.

- Pavement Type: No pavement surface type was specifically excluded from the study. Pavement types on Indiana's two-lane rural state highways include: asphaltic concrete (AC), Portland cement concrete (PCC), and composite (PCC-over-AC). On the county roads studied, pavement types encountered were AC, chip sealed surfaces, natural gravel surfaces, and crushed rock (limestone) surfaces. For the state highways, pavement type was not explicitly considered.

\subsection{OVERVIEW OF THE STUDY APPROACH}

Figure 1-8 illustrates the entire study approach. The study began with a detailed literature survey on the crash trends and factors from published information. The next step was to carry out a survey of law enforcement officials to identify any road locations that are prone to crashes. For county roads, crash and volume data were obtained from Tippecanoe County LTAP office, and data on road width, shoulder width, surface types, and number of curves and driveways, etc. were collected on-site by the Research Team. For state highways, crash, traffic, and inventory data were obtained from INDOT and CATS.

Preliminary descriptive analysis of the data was carried out to identify general trends of the selected variables. Detailed statistical analyses were conducted to develop crash prediction models from which crash reduction factors were derived. Then, for the state highway rural two-lane highways, the cost-effectiveness values of various countermeasures were determined as a function of the countermeasure cost and crash reduction. Also, the crash reduction factors and costs of the countermeasures were used, together with the existing inventory of roads and their geometric deficiencies, to carry out financial needs assessments for various budget and crash costing scenarios for the rural two-lane state highway system. 


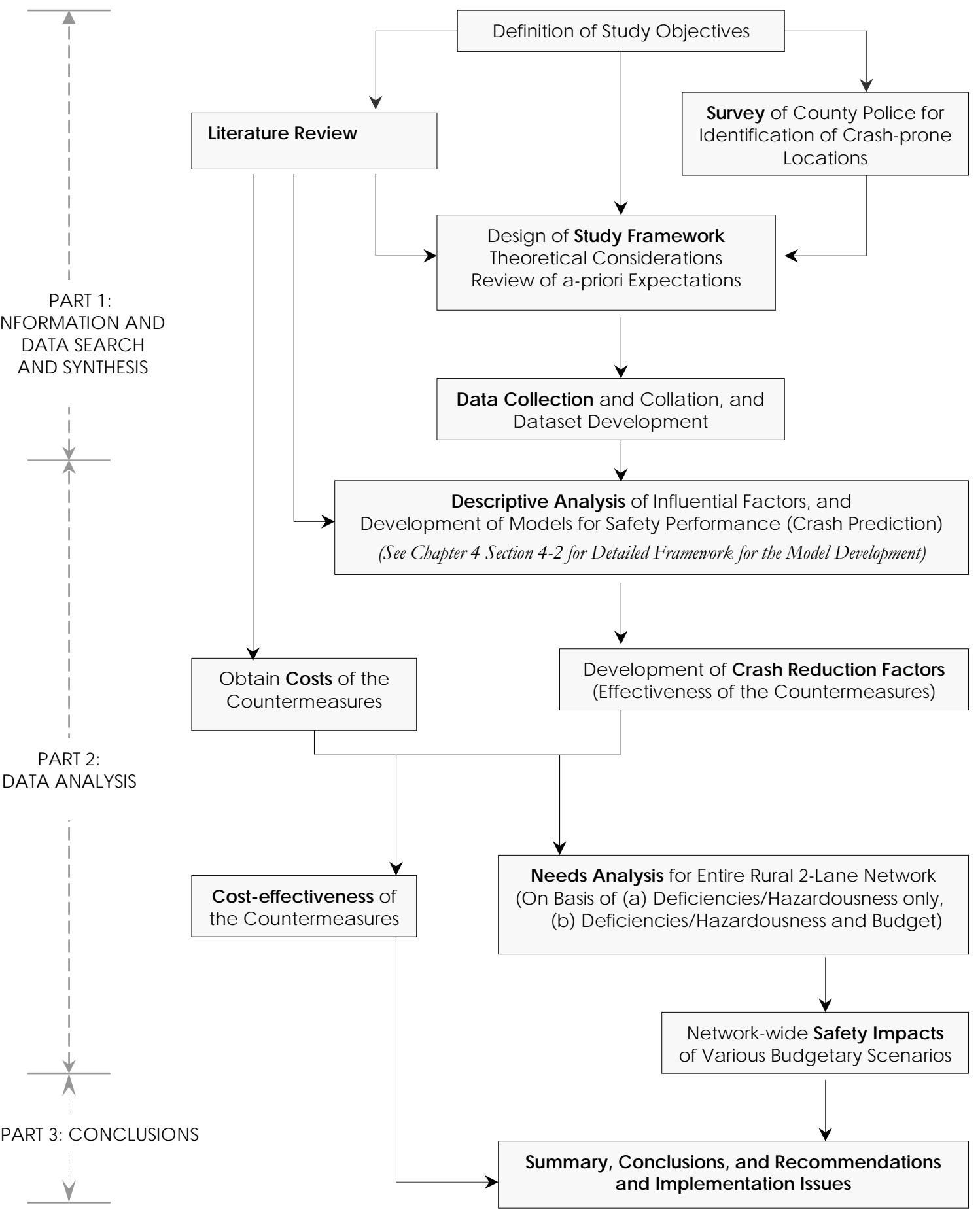

Figure 1-8: Overall Study Approach 


\section{CHAPTER 2 SURVEY RESULTS}

As part of the present study, a survey was developed and sent to sheriffs of the 92 counties in Indiana. These law enforcement personnel have acquired years of valuable experience on crash locations in their jurisdictions and the survey sought to solicit their experiences so that their local perspectives on the identified high-risk areas in each county could be obtained. The survey participants were provided with maps and a questionnaire to assist them in identifying any "trouble" spots (locations of high crash frequencies). Copies of the survey cover letter and the questionnaire are attached as Appendices C1 and C2, respectively. There were 16 responses, and a summary of the synthesized results is presented as Table 3-1.

After receiving the responses, the research team identified the pseudo-codes (PC) for these locations, and used such codes to query crash data from the Indiana Crash database. This way, the road sections with highest number of crashes (per mile) were identified and compared with the findings from the questionnaire survey. It was found that for most of the responding counties, the sections identified by the survey respondents as "trouble spots" did have crash histories higher than similar surrounding sections, but only constituted a very small subset of the overall list of crash-prone sections. 
Table 3-1 Survey Results -- Crash Prone Locations

\begin{tabular}{|c|c|c|c|c|c|c|c|}
\hline County_ID & county & County & PesudoCodes & PC & Key & Street_name & Street_type \\
\hline 902 & 2 & Allen & 75700 & 9075700 & $9075700 \mathrm{C} 2$ & DUPONT & $\mathrm{RD}$ \\
\hline 902 & 2 & Allen & 122700 & 9122700 & $9122700 \mathrm{C} 2$ & HOMESTEAD & $\mathrm{RD}$ \\
\hline 902 & 2 & Allen & 53600 & 9053600 & $9053600 \mathrm{C} 2$ & COLDWATER & $\mathrm{RD}$ \\
\hline 902 & 2 & Allen & 314800 & 9314800 & $9314800 \mathrm{C} 2$ & ABOITE & TR \\
\hline 902 & 2 & Allen & 15200 & 9015200 & $9015200 \mathrm{C} 2$ & BASS & $\mathrm{RD}$ \\
\hline \multirow[t]{2}{*}{910} & 10 & Clark & 159400 & 9159400 & $9159400 \mathrm{C} 10$ & OLD SR 62 & \\
\hline & & & & 9000000 & $9000000 \mathrm{C}$ & & \\
\hline 910 & 10 & Clark & 160001 & 9160001 & $9160001 C 10$ & SR 160 & \\
\hline 910 & 10 & Clark & 143500 & 9143500 & $9143500 \mathrm{C} 10$ & JOHN NOBLE & $\mathrm{RD}$ \\
\hline 910 & 10 & Clark & 125700 & 9125700 & $9125700 \mathrm{C} 10$ & HENRYVILLE/BLUELICK & $\mathrm{RD}$ \\
\hline 910 & 10 & Clark & 125300 & 9125300 & $9125300 \mathrm{C} 10$ & BETHLEHEM & $\mathrm{RD}$ \\
\hline 915 & 15 & Dearborn & 88100 & 9088100 & $9088100 \mathrm{C} 15$ & SR 1 RP 002 & \\
\hline 915 & 15 & Dearborn & 88600 & 9088600 & $9088600 \mathrm{C} 15$ & SR 1 RP 007 & \\
\hline 915 & 15 & Dearborn & 48001 & 9048001 & $9048001 \mathrm{C} 15$ & SR 48 & \\
\hline 915 & 15 & Dearborn & 95200 & 9095200 & $9095200 \mathrm{C} 15$ & SR 350 RP 012 & \\
\hline 915 & 15 & Dearborn & 93100 & 9093100 & $9093100 \mathrm{C} 15$ & SR 56 RP 192 & \\
\hline 923 & 23 & Fountain & 147700 & 9147700 & $9147700 \mathrm{C} 23$ & CR $1400 \mathrm{~N}$ & \\
\hline 923 & 23 & Fountain & 182700 & 9182700 & $9182700 \mathrm{C} 23$ & PORTLAND ARCH & $\mathrm{RD}$ \\
\hline 923 & 23 & Fountain & 176200 & 9176200 & $9176200 \mathrm{C} 23$ & DIXIE BEE & $\mathrm{RD}$ \\
\hline 923 & 23 & Fountain & 186400 & 9186400 & $9186400 \mathrm{C} 23$ & STONE BLUFF & $\mathrm{RD}$ \\
\hline 924 & 24 & Franklin & 86900 & 9086900 & $9086900 \mathrm{C} 24$ & US 52 RP 148 & \\
\hline 924 & 24 & Franklin & 87100 & 9087100 & $9087100 \mathrm{C} 24$ & US 52 RP 149 & \\
\hline 924 & 24 & Franklin & 87200 & 9087200 & $9087200 \mathrm{C} 24$ & US 52 RP 150 & \\
\hline 924 & 24 & Franklin & 87300 & 9087300 & $9087300 \mathrm{C} 24$ & US 52 RP 151 & \\
\hline 927 & 27 & Grant & 134700 & 9134700 & $9134700 \mathrm{C} 27$ & SR 18 RP 096 & \\
\hline 929 & 29 & Hamilton & 49600 & 9049600 & $9049600 \mathrm{C} 29$ & 106TH & ST \\
\hline 929 & 29 & Hamilton & 49700 & 9049700 & $9049700 \mathrm{C} 29$ & 116TH & ST \\
\hline 929 & 29 & Hamilton & 67400 & 9067400 & $9067400 \mathrm{C} 29$ & $141 S T$ & ST \\
\hline 929 & 29 & Hamilton & 55200 & 9055200 & $9055200 C 29$ & FALL CREEK & $\mathrm{RD}$ \\
\hline 929 & 29 & Hamilton & 263100 & 9263100 & $9263100 \mathrm{C} 29$ & SR 238 RP 000 & \\
\hline 929 & 29 & Hamilton & 263200 & 9263200 & $9263200 \mathrm{C} 29$ & SR 238 RP 001 & \\
\hline 929 & 29 & Hamilton & 263300 & 9263300 & $9263300 \mathrm{C} 29$ & SR 238 RP 002 & \\
\hline 929 & 29 & Hamilton & 263400 & 9263400 & $9263400 \mathrm{C} 29$ & SR 238 RP 003 & \\
\hline 929 & 29 & Hamilton & 263500 & 9263500 & $9263500 \mathrm{C} 29$ & SR 238 RP 004 & \\
\hline 929 & 29 & Hamilton & 263600 & 9263600 & $9263600 \mathrm{C} 29$ & SR 238 RP 005 & \\
\hline 929 & 29 & Hamilton & 263700 & 9263700 & $9263700 \mathrm{C} 29$ & SR 238 RP 006 & \\
\hline 929 & 29 & Hamilton & 263800 & 9263800 & $9263800 \mathrm{C} 29$ & SR 238 RP 007 & \\
\hline
\end{tabular}


Table 3-1 Survey Results -- Crash Prone Locations (continued)

\begin{tabular}{|c|c|c|c|c|c|}
\hline County_ID & county & County & PesudoCodes & PC & Key \\
\hline 929 & 29 & Hamilton & 263900 & 9263900 & $9263900 \mathrm{C} 29$ \\
\hline 929 & 29 & Hamilton & 264100 & 9264100 & $9264100 \mathrm{C} 29$ \\
\hline 932 & 32 & Hendricks & 223300 & 9223300 & $9223300 \mathrm{C} 32$ \\
\hline 932 & 32 & Hendricks & 156100 & 9156100 & $9156100 \mathrm{C} 32$ \\
\hline 932 & 32 & Hendricks & 158100 & 9158100 & $9158100 \mathrm{C} 32$ \\
\hline 932 & 32 & Hendricks & 22400 & 9022400 & $9022400 \mathrm{C} 32$ \\
\hline 932 & 32 & Hendricks & 90900 & 9090900 & $9090900 \mathrm{C} 32$ \\
\hline 932 & 32 & Hendricks & 162200 & 9162200 & $9162200 \mathrm{C} 32$ \\
\hline 932 & 32 & Hendricks & 160400 & 9160400 & $9160400 \mathrm{C} 32$ \\
\hline 940 & 40 & Jennings & 43700 & 9043700 & $9043700 \mathrm{C} 40$ \\
\hline 940 & 40 & Jennings & 46200 & 9046200 & $9046200 C 40$ \\
\hline 940 & 40 & Jennings & 7001 & 9007001 & $9007001 \mathrm{C} 40$ \\
\hline 940 & 40 & Jennings & 49400 & 9049400 & $9049400 \mathrm{C} 40$ \\
\hline 940 & 40 & Jennings & 51600 & 9051600 & $9051600 \mathrm{C} 40$ \\
\hline 940 & 40 & Jennings & 34200 & 9034200 & $9034200 C 40$ \\
\hline 943 & 43 & Kosciusko & 99200 & 9099200 & $9099200 \mathrm{C} 43$ \\
\hline 943 & 43 & Kosciusko & 100200 & 9100200 & $9100200 \mathrm{C} 43$ \\
\hline 943 & 43 & Kosciusko & 54100 & 9054100 & $9054100 \mathrm{C} 43$ \\
\hline 943 & 43 & Kosciusko & 63300 & 9063300 & $9063300 \mathrm{C} 43$ \\
\hline 943 & 43 & Kosciusko & 64400 & 9064400 & $9064400 \mathrm{C} 43$ \\
\hline 943 & 43 & Kosciusko & 67100 & 9067100 & $9067100 \mathrm{C} 43$ \\
\hline 945 & 45 & Lake & 221200 & 9221200 & $9221200 \mathrm{C} 45$ \\
\hline 945 & 45 & Lake & 218500 & 9218500 & $9218500 C 45$ \\
\hline 945 & 45 & Lake & 215300 & 9215300 & $9215300 \mathrm{C} 45$ \\
\hline 945 & 45 & Lake & 10600 & 9010600 & $9010600 \mathrm{C} 45$ \\
\hline 945 & 45 & Lake & 22500 & 9022500 & $9022500 \mathrm{C} 45$ \\
\hline 949 & 49 & Marion & 446600 & 9446600 & $9446600 C 49$ \\
\hline 949 & 49 & Marion & 3000 & 9003000 & $9003000 \subset 49$ \\
\hline 949 & 49 & Marion & 450100 & 9450100 & $9450100 C 49$ \\
\hline 949 & 49 & Marion & 450200 & 9450200 & $9450200 C 49$ \\
\hline 949 & 49 & Marion & 87500 & 9087500 & $9087500 C 49$ \\
\hline 949 & 49 & Marion & 114200 & 9114200 & $9114200 C 49$ \\
\hline 949 & 49 & Marion & 327200 & 9327200 & $9327200 C 49$ \\
\hline 953 & 53 & Monroe & 63700 & 9063700 & $9063700 \mathrm{C} 53$ \\
\hline 953 & 53 & Monroe & 19900 & 9019900 & $9019900 \mathrm{C} 53$ \\
\hline 953 & 53 & Monroe & 26700 & 9026700 & $9026700 \mathrm{C} 53$ \\
\hline 953 & 53 & Monroe & 74900 & 9074900 & $9074900 \mathrm{C} 53$ \\
\hline 953 & 53 & Monroe & 93600 & 9093600 & $9093600 \mathrm{C} 53$ \\
\hline 954 & 54 & Montgomery & 13900 & 9013900 & $9013900 \mathrm{C} 54$ \\
\hline 954 & 54 & Montgomery & 47001 & 9047001 & $9047001 \mathrm{C} 54$ \\
\hline 954 & 54 & Montgomery & 61100 & 9061100 & $9061100 \mathrm{C} 54$ \\
\hline 954 & 54 & Montgomery & 70900 & 9070900 & $9070900 \mathrm{C} 54$ \\
\hline 954 & 54 & Montgomery & 136002 & 9136002 & $9136002 \mathrm{C} 54$ \\
\hline 981 & 81 & Union & 14600 & 9014600 & $9014600 \mathrm{C} 81$ \\
\hline 981 & 81 & Union & 18700 & 9018700 & $9018700 \mathrm{C} 81$ \\
\hline 981 & 81 & Union & 101001 & 9101001 & $9101001 \mathrm{C} 81$ \\
\hline 990 & 90 & Wells & 35400 & 9035400 & $9035400 \mathrm{C} 90$ \\
\hline 990 & 90 & Wells & 1001 & 9001001 & $9001001 \mathrm{C} 90$ \\
\hline 90 & 90 & Wells & 6600 & 9006600 & $9006600 \mathrm{C} 90$ \\
\hline
\end{tabular}

\begin{tabular}{|c|c|}
\hline \multirow{2}{*}{\multicolumn{2}{|c|}{$\begin{array}{c}\text { Street_name } \\
\text { SR } 238 \text { RP } 008\end{array}$}} \\
\hline & \\
\hline \multicolumn{2}{|l|}{ SR 238 RP 009} \\
\hline ROCKVILLE & RD \\
\hline \multicolumn{2}{|l|}{ US 36 RP 047} \\
\hline \multicolumn{2}{|l|}{ US 36 RP 065} \\
\hline GREEN & ST \\
\hline AVON & AV \\
\hline \multicolumn{2}{|l|}{ US 136 RP 071} \\
\hline \multicolumn{2}{|l|}{ US 136 RP 055} \\
\hline \multicolumn{2}{|l|}{ SR 3 RP 030} \\
\hline \multicolumn{2}{|l|}{ SR 3 RP 052} \\
\hline \multicolumn{2}{|l|}{ SR 7} \\
\hline \multicolumn{2}{|l|}{ US 50 RP 108} \\
\hline \multicolumn{2}{|l|}{ US 50 RP 128} \\
\hline VERNON & ST \\
\hline \multicolumn{2}{|l|}{ OLD ROAD 30} \\
\hline PACKERTON & RD \\
\hline ARMSTRONG & RD \\
\hline \multirow{3}{*}{\multicolumn{2}{|c|}{$\begin{array}{l}\text { COUNTY FARM } \\
\text { CR } 100 \mathrm{E} \\
\text { CR } 300 \mathrm{E}\end{array}$}} \\
\hline & \\
\hline & \\
\hline $117 \mathrm{TH}$ & AV \\
\hline $101 \mathrm{ST}$ & AV \\
\hline 91ST & AV \\
\hline BELSHAW & RD \\
\hline CEDAR LAKE & RD \\
\hline $25 \mathrm{TH}$ & ST \\
\hline 42ND & TER \\
\hline 42ND & PL \\
\hline 42ND & ST \\
\hline \multicolumn{2}{|l|}{ COUNTRY CLUB } \\
\hline EASTWOOD & DR \\
\hline \multicolumn{2}{|l|}{ EASTWOOD } \\
\hline \multicolumn{2}{|l|}{ OLD SR 37} \\
\hline \multicolumn{2}{|l|}{ CURRY PIKE } \\
\hline FAIRFAX & RD \\
\hline ROGERS & RD \\
\hline VERNAL & PIKE \\
\hline LADOGA & RD \\
\hline \multicolumn{2}{|l|}{ SR 47} \\
\hline \multicolumn{2}{|l|}{$\mathrm{CR} 500 \mathrm{~S}$} \\
\hline \multicolumn{2}{|l|}{ CR $1050 \mathrm{~N}$} \\
\hline \multicolumn{2}{|l|}{ US 136} \\
\hline LIBERTY & ST \\
\hline LIBERTY & AV \\
\hline \multicolumn{2}{|l|}{ SR 101} \\
\hline \multicolumn{2}{|l|}{ CR $1200 \mathrm{~N}$} \\
\hline SR 1 & \\
\hline CR $100 \mathrm{E}$ & \\
\hline
\end{tabular}




\section{CHAPTER 3 LITERATURE REVIEW}

\subsection{INTRODUCTION}

This chapter presents a review of literature on crash prediction modeling, methods for identifying hazardous locations, and methods for safety resource allocation (scheduling of network safety projects over time, with or without budgetary constraints). This discussion serves as a prelude to subsequent chapters that describe the prosecution of similar tasks in the present study. The literature review for individual crash factors was deliberately left out from the present chapter and is rather presented in Chapter 5 where it complements details of these factors at Indiana's rural two-lane state highway system. Finally, the present chapter reviews existing safety management software that can be used by INDOT, LTAP, MPO's and other local Indiana agencies to estimate future crashes at any section, to identify hazardous locations, and to carry out safety resource allocation.

\subsection{CRASH PREDICTION MODELING}

Crash prediction models, typically developed from statistical analysis of historical crash data, are used to predict the expected number of crashes at any specific road segment, and can also be used to derive crash reduction factors that measure the effectiveness of safety countermeasures. Prerequisites to crash modeling include developing a crash database (with roadway characteristics such as traffic volumes, geometric design features, traffic control features, and crash history), selecting an appropriate functional form, and using regression analysis to estimate the values of the model parameters. With regard to the model form and parameter estimation method, a review of available literature revealed that three main model types (Poisson, Negative Binomial, and Non-linear) have been used in the past for crash prediction modeling, as discussed in subsequent sub-sections of this section.

\subsubsection{Poisson Models}

Poisson regression is one of the most suitable techniques for crash prediction modeling because highway crashes are discrete rare events and crash counts are non-negative integer variables. Poisson regression models also provide an easy linkage between crash occurrence and the concept of probability. This is because the number of crashes in a given space-time region can be considered as 
a random variable with probabilities that are Poisson distributed. The general form of crash models derived using a Poisson model is:

$$
A=k L Q^{\beta} \exp \left(\sum \gamma_{i} X_{i}\right)
$$

Where $A$ is the number of crashes in a selected time period, $L$ is the length of the section, $Q$ is the average daily traffic on the section, $X_{\mathrm{i}}$ is the explanatory variable and $k, \beta_{\mathrm{i}}$, and $\gamma_{\mathrm{i}}$ are constants estimated from the modeling process.

The above formulation has been used in a number of studies. Miaou et al. [1993], for example, used a Poisson model to estimate the frequency of truck crashes along a set of highway segments as follows:

$$
\lambda_{i}=\exp \left(0.0818+0.1022 x_{1 i}+0.0949 x_{2 i}+0.0426 x_{3 i}+0.0341 x_{4 i}-0.0263 x_{5 i}\right)
$$

where $x_{1 i}$ is the average daily traffic per lane (in thousands of vehicles), $x_{2 i}$ is the horizontal curvature (in degrees per hundred feet), $x_{3 i}$ equals $x_{2 i}$ horizontal curve length (in miles), $x_{4 i}$ is the deviation of stabilized outside shoulder width per direction from $12 \mathrm{ft}$ (in $\mathrm{ft}$.) and $x_{5 i}$ is the percent trucks in traffic stream. Although the model was developed for truck crashes, it is also applicable to crashes involving other vehicle types.

Another group of researchers, Saccomanno et al. [2001], developed a Poisson model for estimating expected crash frequency along a highway section in southern Italy. The highway section consisted of individual homogeneous segments for which 1993-1999 geometric and crash data were obtained. The developed model is as follows:

$$
\mu_{i}=\exp \left(-1.420+0.003 x_{1}+0.056 x_{2}+0.539 x_{3}-0.018 x_{5}\right)
$$

where $x_{1}$ is the length of section, $x_{2}$ is the number of private driveways in section, $x_{3}$ is the number of major intersections in section, and $x_{5}$ is the change in $85^{\text {th }}$ percentile speed from previous section.

For the Saccomanno model, the "section length" variable was used to represent crash exposure due to uniformity of AADT over the entire road length. Although all the variables in the model were found to be significant at the 5\% significance level, the obtained scale-deviance of 230.35 for the model suggests a fair degree of Poisson overdispersion in the crash data. This implies that a large number of the section-specific crash frequencies remain unaccounted for by the model. Thus the Poisson model form may not provide a complete picture of the long-term crash occurrence at highway segments when overdispersion exists in crash data. This seems to support arguments proffered by critics of the Poisson approach who have suggested that the underlying Poisson assumption that the mean equals the variance is unduly restrictive because in reality, the estimated variance-to-mean ratio typically exceeds unity (that is, 1.0). In the present study therefore, Poisson 
models were not used for the crash prediction modeling except in cases where the numbers of observations are very small, such as fatal crash frequencies.

\subsubsection{Negative Binomial Models}

As noted in the preceding sub-section, a weakness of the Poisson approach is the unduly restrictive assumption that the mean and the variance of crash distribution are equal. In most crash data, however, it is seen that the variance is greater than the mean, giving rise to the overdispersion phenomenon. To address this problem, the Negative Binomial model, a variant of the Poisson, has often been proposed for crash prediction modeling. The Negative Binomial model allows for additional variance representing the effect of omitted variables.

In a study for Indiana, Brown et al. [1998] developed crash prediction models for crash rates on road segments based on geometric and access control characteristics. The data used for that study was from the Indiana crash and road inventory database covering the period 1991 to 1995. Using negative binomial regression analysis, separate crash prediction models for three different crash severity categories were obtained - all crashes, PDO crashes, and fatal+injury crashes, as shown as Equation 3-4:

$C R A S H=0.494 \cdot L E N G \cdot Y R S \cdot A A D T \exp (0.0285 \cdot A C-0.631 \cdot S H L D R+2.52 \cdot P S-0.748 \cdot T W L T L-0.604 \cdot N O M E D O)$ $P D O=0.374 \cdot L E N G \cdot Y R S \cdot A A D T \exp (0.0261 \cdot A C-0.669 \cdot S H L D R+2.63 \cdot P S-0686 \cdot T W L T L-0.684 \cdot N O M E D O)$ $F A T I N J=0.127 \cdot L E N G \cdot Y R S \cdot A A D T \exp (0.0325 \cdot A C-0.525 \cdot S H L D R+2.28 \cdot P S-0.865 \cdot T W L T L-0.493 \cdot N O M E D O)$

Where:

$C R A S H$ is the total number of crashes on the segment, PDO is the number of property-damage-only crashes on the segment, FATINJ is the number of fatal and injury crashes on the segment, LENG is the length of the segment in kilometers, YRS is the number of years of available crash data, $A A D T$ is the Annual Average Daily Traffic (in thousands), $A C$ is the access density (number of access points per $\mathrm{km}$ ), SHLDR is a dummy variable to indicate presence of outside shoulder, TWLTL is a dummy variable to indicate presence of two-way left-turn lane on segment and NOMEDO is a dummy variable to indicate presence of a median.

In the Brown study, overdispersion factors of 1.049, 1.157, and 1.105 were obtained for total crashes, fatal/injury and PDO models, respectively. The study concluded that the number of crashes on roadway segments increases as the access density and proportion of signalized access points increase. Also, the presence of an outside shoulder, two-way left-turn lane, or median without openings between signals were found to be associated with lower crash numbers. 
For intended use in the crash prediction module of FHWA's Interactive Highway and Safety Design Model (IHSDM), rural two-lane highway crash prediction models were developed by Vogt and Bared [1998]. The model was developed using an "extended" negative binomial regression analysis of data from 619 rural two-lane highway segments in Minnesota and 712 roadway segments in Washington. An advantage of the extended negative binomial model is that it permits local variation along a roadway to be taken into account so that crashes occurring on sub-segments with invariant geometric conditions are considered separately. Considered a better alternative to summing all the local effects or averaging them over an entire segment, the ENB modeling technique offers scale independence of the model form thereby allowing segment decomposition into sub-segments or aggregate adjacent segments without any changes to the model form. The variables found to have significant influence on crashes were lane width, shoulder width, roadside hazard rating, driveway density, degree of horizontal curvature, vertical grade and traffic exposure. The model had a high predictive power and explained $65 \%$ of the variation in the dataset. An over-dispersion factor of 0.33 was obtained for the model suggesting a good fit. Other variables that were investigated included posted speed limit, truck percentage, and intersection density (i.e., number of intersections per mile). Posted speed limit was found to be negatively correlated with crash frequency while truck percentage and intersection density were positively correlated with crash frequency. None of these three variables were statistically significant at $95 \%$ level of confidence.

In a subsequent review of the Vogt and Bared model, Harwood et al., [2000] averred that the model could be calibrated and adapted for use in all the states. The present study adopted a similar but modified technique: as much as possible, sections were chosen such that within each section there was reasonable constancy of geometric features. Then each section was treated as an observation in the modeling dataset. The present study considered the use of the Negative Binomial model for injury, PDO, and "total" crash analysis. For fatal crashes, the Poisson model was used.

\subsubsection{Non-Linear Models}

Zegeer et al. [1986] developed non-linear crash models using crash, traffic, roadway, and roadside data collected on 1,944 road sections covering 4,951 miles of rural two-lane roads in seven states. The purpose of that study was to quantify the benefits of lane widening, shoulder widening, side slope flattening etc. The model was developed for single vehicle crashes, head-on crashes, and sideswipe crashes (of same and opposite directions) and is given as follows:

$A=0.0019 \times A D T^{0.8786} \times 0.9192^{W} \times 0.9316^{P A} \times 0.8824^{U P} \times 1.2365^{H} \times 0.8822^{T 1} \times 1.3221^{T 2}$ 
where $A D T$ is the average daily traffic, $W$ is the lane width in feet, $P A$ is the average width of the paved shoulder in feet, UP is the average width of the unpaved shoulder, $H$ is the median or roadside hazard rating ( 1 to 7$), T_{1}$ is equal to 1 if the terrain is flat and 0 otherwise, and $T_{2}$ is equal to 1 if the terrain is mountainous and 0 otherwise.

The least-squares approach was used to estimate the regression coefficients. This was based on the assumption that the residuals would follow a normal or log-normal distribution. However, because crash distributions are typically skewed to the right, normality of the residuals may not be a tenable assumption for crash prediction models.

Forkenbrock et al. [1997] developed a semi-log regression equation using roadway and threeyear crash data on 17,767 two-lane road sections and four-lane (non interstate) rural primary road segments in Iowa. The semi-log model form was chosen for this crash-rate prediction model because almost a third of the road segments had no crashes over the 3-year period. The crash rate model developed in that study is shown as Equation 3-6:

$C R=0.517 \times 0.972^{\text {PSR }} \times 1.068^{T C U R V} \times 1.179^{\text {PASSRES }} \times 1.214^{A D T L N} \times 0.974^{R T S H} \times 0.933^{L N} \times 1.051^{T G R A D}$

Where:

$C R$ is the crash rates per million vehicle miles traveled, PSR is the present pavement serviceability rating, TCURV is the degree of curvature of the sharpest curve on the section, PASSRES is the passing restrictions ( 1 if present, 0 if none), $A D T L N$ is the average daily traffic in thousands per lane, RTSH is the width of right shoulder, $L N$ is the number of lanes ( 1 for 4 lanes and 0 for two-lane segments), and TGRAD is the grade of the steepest slope in the segment.

The estimated coefficients of all variables (with the exception of PSR and LN) were found to be statistically significant at the 0.001 level. The major limitation of their model is the use of crash rates as the response variable for the model, thus implicitly assuming that there exists a linear relation between crash frequency and traffic exposure. Also, there are questions over the use of a variable (that is, traffic volume) in the set of independent variables as well as in the formulation of the dependent variable.

\subsubsection{Empirical Bayesian Method}

The use of the Empirical Bayesian (EB) method for crash prediction seems to have become widely accepted as one that yields the most unbiased estimate of the expected crash frequency. The EB method increases the precision of crash frequency estimation and corrects for the regression-tomean bias [Hauer et al., 2002]. The EB model assumes that the number of observed crashes on a 
road section or intersection is distributed according to the Negative Binomial distribution. Using a weight factor, the historical crash record and the expected crash frequency are combined to obtain a more accurate estimate of the safety performance at a location. The weight factor depends on the magnitude of the crash record and on the reliability of the crash prediction models. The Bayesian adjusted/estimated number of crash involvement per year is expressed as a combination of the estimated/predicted number of crashes, $E(m)_{i}$ and the observed number of crashes $\left(X_{\mathrm{i}}\right)$ as follows:

$\varepsilon_{i} W_{i} E(m)_{i}+\left(1-W_{i}\right) X_{i}$ and

$W_{i}=\frac{1}{1+E(m)_{i} / k}$

where $W_{\mathrm{i}}$ is the weight factor, $E(m)_{i}$ is the expected number of crashes at location $\mathrm{i}, X_{\mathrm{i}}$ is the number of observed crashes at location $i$, and $k$ is the over-dispersion factor.

Hauer et al. [2002] demonstrated the application of the EB method using an "abridged" and "full" procedure. The "full" version utilized a longer history of crash and traffic exposure. The study suggested that because the full procedure utilizes a higher number of crash counts, its estimate of crash frequency may be considered more reliable than that of the abridged procedure.

\subsection{METHODS FOR IDENTIFYING HIGH CRASH LOCATIONS AT RURAL TWO-LANE HIGHWAYS AND COUNTY ROADS}

After predicting crash frequency at a number of rural two-lane road sections, safety analysts typically proceed to the next step of identifying sections that may be considered "high crash locations" or "hazardous" and therefore are high priority candidates for safety intervention. Identifying the deserving locations for some safety improvement is a vital component of any safety improvement program due to the limited resources available for such programs. The literature survey showed that several methods for this task have been suggested by past researchers. A review of some of the important findings that cover any road type (and may be adapted for rural two-lane networks) is discussed below.

\subsubsection{Potential-for-Crash Reduction Method}

McGuigan [1981] introduced the concept of "potential crash reduction" as a criterion for identifying candidate locations. The potential crash reduction for a location was computed as the difference between observed crash count and expected crash frequency obtained from a multivariate crash 
prediction model. According to the researcher, this value represents the size of potential annual crash reduction for a given location. In subsequent studies, critics of the McGuigan approach suggested that the high random crash count is not a reliable measure of the current safety level. In a bid to eliminate the randomness associated with crash counts, Persaud [1999] adopted a method similar to McGuigan's but used the EB estimate instead of the observed crash counts. Then critics of the Persaud approach contested the assumption that the expected crash frequency is the level to which crashes can be reduced. In an improvement to the McGuigan and Persaud methods for hazardous location identification, Tarko et al. [1996] suggested that the potential crash reduction should be estimated as the difference between the expected crash frequency and the minimum crash frequency. According to the authors, the minimum crash frequency represents the best level of safety achievable through safety improvements and could be estimated from a multivariate regression equation that incorporates only uncontrolled local factors such as traffic volume.

\subsubsection{Use of Empirical Bayesian Method}

The use of the Empirical Bayesian (EB) method for identifying high-crash locations was first suggested by Abbess et al. [1981] who argued that the true crash rate at a high-crash location could be regarded as a random variable having a probability distribution. According to the researchers, there could be two types of such probability distribution: (i) the prior distribution that involves the distribution of the true crash rate for the period before data becomes available, and (ii) posterior distribution, which involves the distribution of the true crash rate for the period after data becomes available. The authors also demonstrated how the use of the EB method for safety analysis automatically corrects for the regression-to-the-mean effect. In a subsequent research study, Higle and Witkowski [1988] used an EB estimate of crash rate to identify candidate locations from a list of specific highway facilities in Arizona. A location was considered "hazardous" if there was significant probability that the expected crash rate at the location exceeded a specified critical crash rate value. The critical rate is based on the assumption that the number of crashes at a location is Poisson distributed. Another researcher, Hauer [1992] applied the EB method to identify candidate locations using expected crash frequencies rather than expected crash rates for the hazardous location identification process, arguing that a location may be considered hazardous if there is a high probability that the expected crash frequency exceeds a predefined critical crash frequency. 


\subsubsection{Sites-With-Promise (SWiP) Method}

The term "sites-with-promise" is used to describe candidate locations that have a good chance of being in genuine need of remedial action and are also capable of being improved in the most cost effective manner [Hauer, 1996]. The SWiP method involves an initial network screening to identify all candidate locations on the network that deserve some safety improvement. This is followed by a more detailed engineering study to formulate cost-effective projects for implementation at each location. The sites are then prioritized in descending order based on the cost-effectiveness of the identified projects. The approach is intended to guard against poor crash predictions due to the randomness of crash counts [Hauer et al., 2002].

Hummer et al. [2003] applied the SWiP method to identify "promising" sites using data from two counties in Tennessee. The study outlined the application of the inventory method to identify "promising" sites on rural two-lane highways in seven steps as follows:

1. Select population of highway sites.

2. Divide sites into bridges, horizontal curves and tangent highway segments.

3. Predict annual crash frequency at each site.

4. Estimate cost of installing each possible countermeasure at each site.

5. Apply collision reduction factors for each possible countermeasure.

6. Calculate the cost-effectiveness of each possible countermeasure at each site.

7. Identify the most "promising" sites in the study area.

At the crash prediction step of their methodology, Hummer et al. used crash prediction models developed by Turner [1984], Zegeer et al. [1991], and Vogt and Bared [1998]. A fairly comprehensive list of countermeasures was then developed for these sites. The unit costs of these countermeasures were obtained from the North Carolina Department of Transportation while crash reduction factors were averaged using data from the Federal Highway Administration and California Department of Transportation. The cost-effectiveness of the countermeasure at each site was evaluated as the amount of dollars spent per annual number of crashes saved and the sites were ranked in ascending order of dollars spent per annual number of crashes saved. The study also found that the mean hazard rating of hazardous sites identified with the traditional methods were significantly greater than the mean hazard rating of sites identified using the "sites-with-promise" method. 


\subsubsection{Lifetime Highway Risk Method}

Ossenbruggen [1998] used the principle of individual lifetime risk to develop a method for identifying hazardous highway locations. This method, which considers both fatality and injury crashes, is based on the basic principles of probability and expected value theory. A "highway risk" was defined as the expected number of fatal crashes per year for a given highway location, and expresses such risk as follows:

$\mathrm{R}=A D T^{*} \theta$

Where:

$\theta$ is the probability that an individual will be killed in a crash within a given year and $A D T$ is the average daily traffic at a given location. If the fatal crash count for a given location exceeds the expected number of fatal crashes $R$, then the location is classified as "hazardous". Otherwise, the location is considered "safe". The probability that an individual will experience a fatal crash over the individual's lifetime, $\theta$, is given by the model:

$\theta=1-\exp \left(-\eta^{*} \omega\right)$

where $\eta$ is the probability that an individual will be killed in a single motor vehicle trip and $\omega$ is the mean number of trips made by an individual in a lifetime.

This relation was derived from the following assumptions: (i) the trip number in which an individual experiences a fatality follows a geometric distribution with a probability of $\omega$, (ii) the number of trips that an individual makes in a lifetime is assumed to be a Poisson random variable with a mean of $\eta$.

Using principles from public health regulation and the public's perception of highway risk, the value of $\theta$ was estimated as 0.0000143 . It was assumed that the same safety standard applied to all highway system categories. Using national crash counts, the study also showed that the determined value of lifetime risk is a rather rigorous standard because attaining that goal would involve a six-fold decrease in the total number of fatal collisions. According to the researchers, the life highway risk method for identifying hazardous highway locations is consistent with others used in practice.

\subsection{RESOURCE ALLOCATION METHODS}

Like all highway agencies, the Indiana DOT, LTAP, and other local agencies in Indiana develop or seek to develop programs or schedules that specify what safety improvements should be carried out, at which locations, and at which year. A literature search of available resource allocation models for safety programming revealed three main methods: linear programming, integer programming, and dynamic programming. A brief discussion of these methods is provided in subsequent sections. 


\subsubsection{Linear and Integer Programming}

Linear programming (LP) defines a class of problems in which the decision variables are nonnegative, the objective function for selecting the best values of the decision variables is a linear function of these variables, and the constraints (e.g., resources) can be expressed as linear equations or inequalities. The most widely-used computational algorithm for LP's is the Simplex method. Although the LP method is the most common method of mathematical programming, its application for the selection of safety measures for specific locations has not yet seen extensive application by highway safety agencies.

Integer programming is a type of linear programming problem in which some or all of the decision variables are restricted to integer values. Compared to linear programming problems, the solution of integer programming problems involves a higher level of computational effort and time, and can be obtained using the branch-and-bound method. Recent research efforts have provided software tools capable of solving large-scale highway safety problems using this method. Using integer programming, Kaji and Sinha [1981], Sinha et al. [1981], and Pal and Sinha [1998] developed resource allocation methodologies for highway safety improvements in Indiana for cost-effectiveness maximization. This technique was also used by Harwood et al. [2003] to develop a framework for the system-wide optimization of resurfacing and safety improvements under $3 \mathrm{R}$ projects.

\subsubsection{Dynamic Programming}

Dynamic programming is an optimization technique that transforms a multi-stage decision problem into a series of one-stage decision problems. The three possible levels of dynamic programming are:

1. Single-stage (used to evaluate a single project with several alternatives)

2. Multi-stage (involves selection among several projects with several alternatives each)

3. Multi-stage with a time factor (used where several alternatives are considered and various time periods are involved)

Basic inputs for a dynamic programming model comprises: (i) initial costs, operating costs and maintenance costs for each project alternative, (ii) crash reduction benefits for each project alternative and (iii) budget available for improvements. The main advantage of the dynamic programming model is that it is responsive to changes in budget, inflation rates, cost of materials, and other inputs. However, its main disadvantage is the complexity of its computation [FHWA, 1981]. This method is used at Kentucky and Alabama DOT's for resource allocation. In presenting an update to the dynamic programming procedure used in Alabama, Brown et al. [1990] developed a 
"branch-and-bound" technique to handle larger sets of project data more efficiently while ensuring that optimality is guaranteed.

\subsection{EXISTING SOFTWARE THAT CAN BE USED FOR SAFETY ANALYSIS AT RURAL TWO-LANE ROADS}

As stated in its study objectives (Chapter 1), the present study seeks to identify influential crash factors, develop models for crash prediction purposes, identify hazardous locations, develop crash reduction factors for safety countermeasures, assess the cost-effectiveness of countermeasures, develop a priority listing of safety investments, and developing a program for safety investments for the rural two-lane road network at state highway and county level. This section describes existing software for safety analysis and management that were reviewed for possible application in the present study. It should be noted however, that rather than resorting to existing data and models in existing software packages, the present study collected data and carried out these procedures in a manner that is consistent with procedures found in such software packages. The present study also examined how underlying models in these packages could be updated using the present study results for more reliable crash prediction, hazardous location identification, updating of crash reduction factors, and development of safety investment programs.

\subsubsection{Interactive Highway Safety Design Model (IHSDM)}

The Interactive Highway Safety Design Model (IHSDM) is a suite of software analysis tools for evaluating safety and operational effects of highway geometric design decisions. The IHSDM is structured into six evaluation modules. Each model provides different measures of the expected safety performance of an existing or proposed highway geometric design. The modules are described below:

- Policy Review Module - This module checks a design against relevant highway geometric design policies, and highlights design elements that deviate from quantitative policy values. The module organizes the policy checks into four categories: cross section, horizontal alignment, vertical alignment, and sight distance.

- Crash Prediction Module — This module provides quantitative safety performance measures. The module estimates the frequency and severity of crashes that should be expected on a roadway by considering its geometric design and traffic characteristics. The crash prediction algorithm combines base models and crash modification factors. The base models estimate the total crash frequency on a roadway segment or at an intersection under base geometric 
conditions and actual traffic conditions. The crash modification factors adjust the base model estimates for individual geometric design dimensions and traffic control features.

- Design Consistency Module - This module assesses conformity with drivers' speed expectations. The module quantifies the magnitude of speed inconsistencies through a speed-profile model that estimates 85th percentile, free-flow, passenger vehicle speeds at each point along a roadway and compares it with the assumed design speed. Design consistency evaluations are used for diagnosing potential safety issues on existing highways and for quality assurance checks of both preliminary and final alignment designs.

- Driver/Vebicle Module — The Driver/Vehicle Module predicts driver speed and path along a roadway and their effects on vehicle dynamics. This module consists of a driver performance model linked to a vehicle dynamics model. This module can be used to diagnose safety issues on existing roadways and to check both preliminary and detailed geometric designs.

- Intersection Diagnostic Review Module - This module evaluates design elements such as intersection sight distance, intersection angle, corner radius, and turn-lane presence and design that influence the safety performance of at-grade intersections. The module performs a systematic review of intersection design features, highlights potential safety and operational concerns, and identifies alternative treatments to mitigate those concerns.

- Traffic Analysis Module - This module uses a traffic simulation model [Leiman et al., 1998] to estimate the operational effects of road designs under current and projected traffic flows. The module provides information on travel time, delay, traffic conflicts, and other operational measures related to safety. This module can be used to diagnose operating conditions that affect safety on both existing roadways and proposed designs.

The main advantage of the IHSDM is that it includes a traffic simulation module that enables the analyst to evaluate the impact of safety improvements on traffic flow characteristics of the road section. However, the major drawback of the IHSDM is that it is a project-level analysis tool and its current version has limited application to only certain classes of roads. It can only analyze a single road section at a time and hence not capable of direct network optimization. For example, IHSDM, in its current form, cannot be used to directly identify and prioritize multiple sections on a road network for improvement. The package, however, can be used to evaluate and compare the impacts of alternative safety improvement projects at a given location. As such, the IHSDM cannot be used as a stand-alone tool for safety management but can serve as a complementary tool for this purpose. 


\subsubsection{Resurfacing Safety Resource Allocation Program (RSRAP)}

The resurfacing safety resource allocation program is a software tool developed by Harwood et al. [2003] that allows highway agencies to incorporate safety considerations into pavement resurfacing (3R) projects. This tool selects the safety/operational improvements at a given set of locations already scheduled for resurfacing at a specific year; or selects the projects that should be resurfaced and safety improvements that should be implemented given a larger set of candidate locations. This software tool is appropriate for a highway agency that wants to maximize the net benefits from the combined resurfacing and safety improvement program. RSRAP consists of a Microsoft Access database application that utilizes Microsoft Excel's Solver add-in for execution of the optimization process. A drawback of the current version of RSRAP is that it considers a rather limited set of safety improvement projects - certain improvement types, such as guardrail installation and rumble stripping are not directly considered.

\subsubsection{INDOT's Safety Management System Software Package (SMS-IN)}

The Indiana Safety Management System (SMS) software package, SMS-IN, is a Windows-based program that follows the framework illustrated in Figure 3-1 (Lamptey et al., 2004). The software user can select any subset of a highway network for analysis. The underlying database for the current SMS-IN version does not include county roads, but can be modified to include such networks. Each of the six procedures described in the SMS-IN framework constitute a module in the software and can be executed in that order. The results from any module can be used as the input for the subsequent module. However each module can be executed independently from each other. The software also utilizes default values of geometric standards, crash costs, safety improvement projects, etc. All default values can be updated by the user. SMS-IN selects alternative safety improvement projects for each candidate location and performs economic evaluations using Benefit-Cost Ratio (BCR), Cost Effectiveness (CE), or Net Present Value (NPV). Integer optimization is then carried out using the CPLEX ${ }^{\circledR}$ Solver included in the OptiMax component library to select the optimal mix and timing of safety improvement projects for the candidate locations. The software also generates various reports and graphs of the data input and analysis output. SMS-IN has updatable crash prediction equations for all highway classes. For purposes of the present study, the rural two-lane road crash equations existing in the software were replaced by those developed in the present study and used for future crash prediction, development of crash reduction factors, hazardous location identification, and development of network-level safety programs and needs assessments. 
SMS-IN was developed as a stand-alone program using Microsoft Visual Basic.Net platform. The software uses the OptiMax 2000® component library from Maximal software for the optimization routines which allows Mathematical Programming Language (MPL) models to be seamlessly and directly integrated into object-oriented programming languages such as Visual Basic.

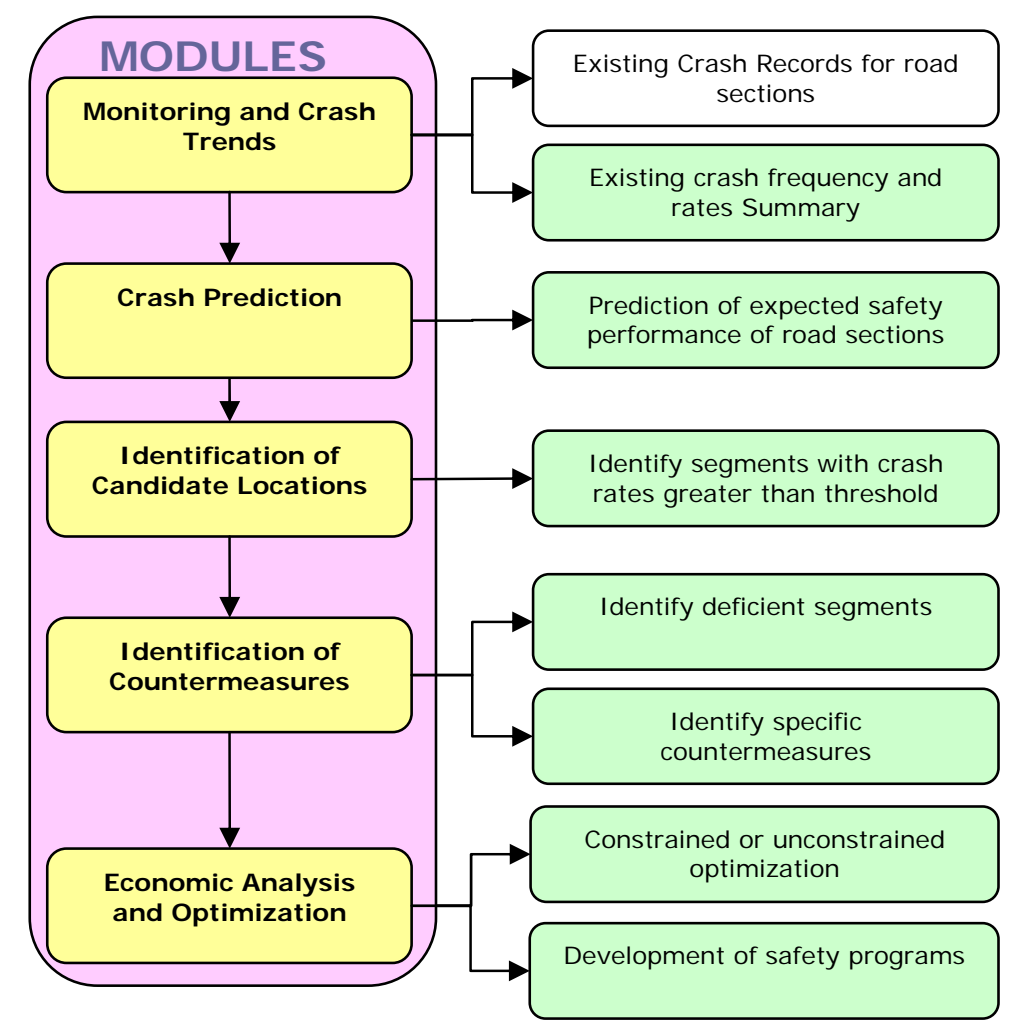

Figure 3.1 Modules and Tasks Addressed by the Indiana Safety Management System Software

\subsection{CHAPTER SUMMARY}

In this chapter, a review of relevant literature was presented. This included crash prediction modeling, methods of identifying hazardous locations, and methods for safety resource allocation (scheduling of network safety projects over time). Subsequent chapters present details of study tasks that addressed crash prediction modeling, hazardous location identification, and resource allocation for the rural to lane state highways and county roads in the state of Indiana. The chapter also reviewed existing safety management software that can be used by INDOT, LTAP and other local agencies to estimate future crashes at any rural two-lane road section, to identify hazardous locations, 
and to carry out safety resource allocation for such networks. In this chapter, the literature review for crash factors was deliberately excluded because that subject is presented in a subsequent chapter (Chapter 5) where it complements descriptions of existing crash factors at Indiana's rural two-lane state highway system. 


\section{CHAPTER 4 ANALYTICAL FRAMEWORK}

\subsection{INTRODUCTION}

This chapter discusses the various framework, methodologies, and underlying theories used in developing crash prediction equation and crash reduction factors, and network-level needs assessments. The methodologies were designed not only to address the study objectives but also to help facilitate reliable data analysis and interpretation that is critical for studies of this nature.

\subsection{DEVELOPMENT OF CRASH PREDICTION MODELS AND CRASH REDUCTION FACTORS}

Figure 4-1 presents the framework used for the development of crash prediction equations. Crash reduction factors were established from the crash prediction models.

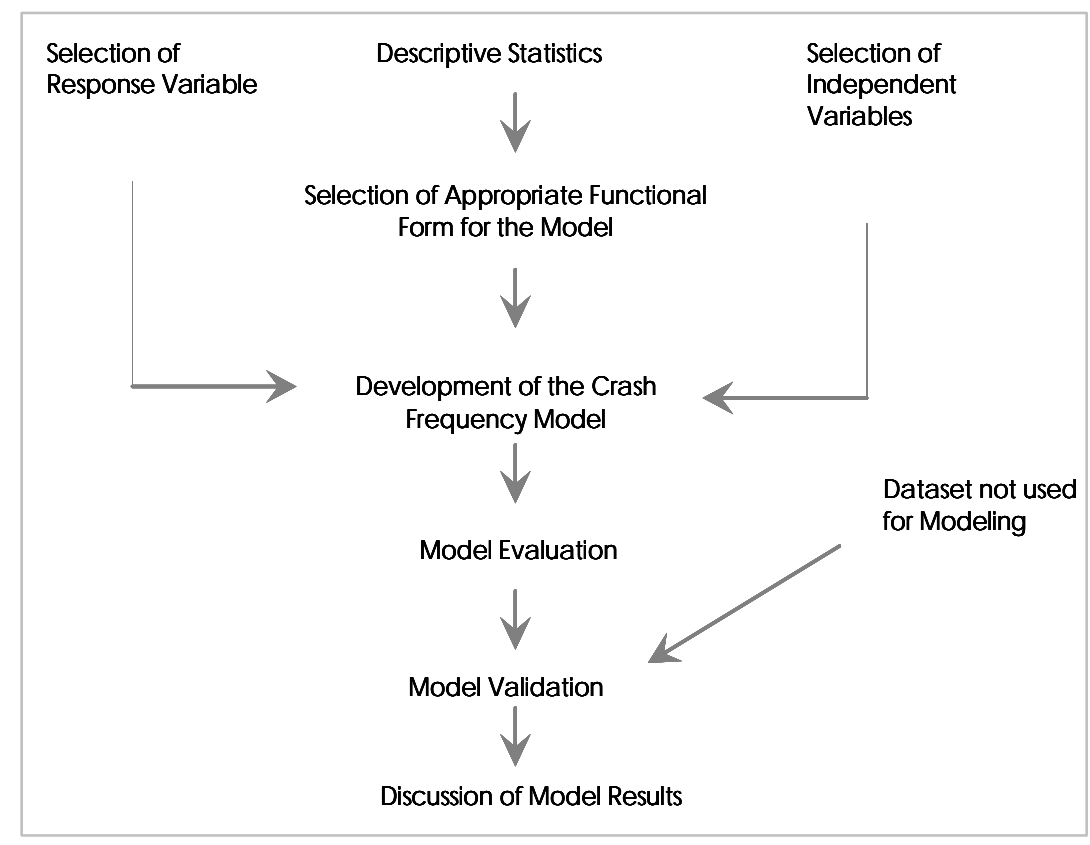

Figure 4-1: Framework for the Model Development Only 


\subsubsection{Choice of Response Variable for the Models}

On the basis of the form of response variable, past studies on roadway crash prediction can be broadly classified into two groups: those that used crash rates and those that used crash frequencies.

Crash Rates: Some researchers have used crash rate (expressed as crashes per unit exposure, such as crashes per mile, crashes per traffic volume, or crashes per vehicle-miles-of-travel) as the response variable for their crash prediction models. This practice implicitly assumes a linear relationship between traffic exposure and number of crashes - an assumption that is not necessarily true. In fact, recent research has shown that number of crashes is typically related to exposure in ways that suggest non-linearity of this relationship. For example, crashes may not be doubled whenever traffic volume is doubled, and therefore the use of a response variable such as crashes per AADT, which inherently assumes otherwise, is likely to yield incorrect results. Similarly, a section that is twice as long as another otherwise similar section may not experience twice the number of crashes. So the use of "crashes per mile" as a response variable which implicitly assumes that doubling road length leads to doubled crashes, may lead to biased models. The same effect is seen when other exposure parameters are used to formulate a "crash per exposure" response variable, such as crashes per AADT or crashes per VMT.

Crash Frequencies: In recent times, the trend has been to use absolute number of crashes as the response variable [Bernardo and Ivan, 1997] by transferring the traffic exposure part of the response variable to the right side of the model equation to serve as an explanatory variable. Consideration of the traffic exposure variables as independent variables (and excluding them from the dependent variable formulation) provides some flexibility in the hypothesized relationship between crashes and exposure (length, traffic volume, VMT) and therefore allows for estimating the exact nature of such relationship (linearity or otherwise). In the present study therefore, it was sought to obviate the biases inherent in assumptions associated with the use of "crashes per mile", "crashes per AADT", or "crashes per VMT" response variables. As such, the variables section length and traffic volume were represented in the model equations not as a denominator to crash frequencies, but rather as independent variables (with power values not constrained to unity, but left flexible for estimation on

the basis of available data). As such, the dependent variable of the crash prediction models shown in the results chapter was crash frequency. 


\subsubsection{Choice of Independent Variables for the Models}

The independent variables for developing the crash prediction model can be categorized as follows:

a. Exposure: One of the most important independent variables affecting crashes at rural two-lane roads is the volume of vehicular traffic (often represented by AADT) or volume of travel (often represented by VMT). Travel is often expressed as a product of section length of a roadway and observed vehicle count on the same roadway.

b. Geometric Characteristics: Road geometry is also an important variable that affects crash occurrence on rural two-lane roads. Key geometric characteristics such as shoulder width and lane width are given due consideration in the present study. Curve features that were considered at various stages of the analysis include curve density (number of curve sections per mile), degree of curve, radius of curve, and length of curve. Geometric variables that were considered include the following:

- Lane width

- Shoulder width

- Section length

- Horizontal curvature

- Vertical alignment

c. Other Characteristics. These include:

- Shoulder type

- Pavement surface condition or friction

\subsubsection{Choice of Model Form}

The choice of model form for statistical modeling typically depends on the nature of the response variable and its relationships with the explanatory variables. Linear models have been found to be unsuitable for crash modeling because of the non-negative and discrete nature of crash data. Secondly, the error terms of crash data are typically not normally distributed as implicitly assumed in linear modeling [Jovanis et al., 1985]. Model forms such as the Poisson and negative binomial models have become well accepted for modeling discrete rare events such as crash occurrence [Miaou et al., 1993].

The Poisson Model for Predicting Fatal Crash Frequency: For fatal crashes only, the Poisson model was used. The general form of the Poisson probability function is given by

$$
P\left(Y=y_{i}\right)=\frac{\mu_{i}^{y_{i}} e^{-\mu_{i}}}{y_{i} !}
$$


where:

$P\left(Y=y_{i}\right)=\quad$ Probability of $y_{i}$ crashes on section $i$, in a selected time period,

$\mu_{i} \quad=\quad$ Mean number of crashes on section $i$ in a selected time period.

The Poisson and Negative Binomial models assume that crashes occurring on a particular roadway or intersection are independent of one another and the mean number of crashes per unit time is characteristic of the given site and of other sites with the same properties. The mean is assumed to depend on physical characteristics of the road section and is given by:

$$
\mu_{i}=\exp \left(\beta_{0}+\sum_{j=1}^{n} \beta_{j} x_{i j}\right)
$$

where

$\begin{array}{lll}\mu_{i} & = & \text { Expected number of crashes on section } i \text { in a selected time period, } \\ x_{i j} & = & \text { Explanatory variables, } \\ \beta_{i,} & = & \text { Coefficients to be estimated. }\end{array}$

The Poisson regression is estimated using the standard maximum likelihood methods by applying the likelihood function.

$$
L(\beta)=\sum_{i=1}^{n}\left(y_{i} \log \mu_{i}-\mu_{i}-\log y_{i}\right)
$$

where

$\beta=\quad$ Vector of coefficients $\left(\beta_{0}, \beta_{1}, \ldots ., \beta_{\mathrm{n}}\right)$,

$y_{i}=$ Observed crash count on section $i$,

$\mu_{i}=$ Expected number of crashes on section $i$.

The value of $\beta$ that maximizes the $\log$ likelihood function is the estimated coefficient vector $\beta$ and the estimated value of $\mu_{i}$ is the expected crash frequency. Further discussions on the model estimation methodology are provided in Section 4.2.6.

The Negative Binomial Model for Predicting Frequency of Other Crash Severity Types: For crash severity types other than fatal, the negative binomial form (a generalized form of the Poisson model) was used. The negative binomial distribution adds to the variance a quadratic term that represents the overdispersion. This allows for extra Poisson variation due to variables not included in the model. 
The functional form used to obtain the expected crashes is shown in Equation 4-1b, and this is then used in the negative binomial model that is given as follows:

$$
P\left(Y=y_{i}\right)=\frac{\Gamma\left(\frac{1}{\alpha}+y_{i}\right)}{\Gamma\left(\frac{1}{\alpha}\right) \cdot y_{i} !} \cdot\left(\frac{1}{\left(1+\alpha \mu_{i}\right)}\right)^{\frac{1}{\alpha}} \cdot\left(1-\frac{1}{1+\alpha \mu_{i}}\right)^{y_{i}}
$$

where

$\alpha=$ Overdispersion parameter estimated by the maximum likelihood technique,

$\Gamma=$ Gamma function.

Unlike the Poisson model where the variance is restricted to be equal to the mean, the variance of the negative binomial model is given by:

$$
\operatorname{Var}(Y)=\quad \mu_{i}\left(1+\alpha \mu_{i}\right)
$$

The negative binomial regression model is estimated using the standard maximum likelihood methods by applying the likelihood function shown as Equation 5-6:

$$
L L(\beta)=\sum_{i=1}^{n}\left[\left(\sum_{j=0}^{y_{i}} \log (1+\alpha \cdot j)\right)-\log \left(1+\alpha \cdot y_{i}\right)+y_{i} \cdot \log \mu_{i}-\left(y_{i}+\alpha^{-1}\right) \log \left(1+\alpha \cdot \mu_{i}\right)-\log \left(y_{i} !\right)\right]
$$

Where

$$
\begin{array}{lll}
\alpha & = & \text { Overdispersion parameter, } \\
\beta & = & \text { Vector of coefficients }\left(\beta_{0}, \beta_{1}, \ldots, \beta_{\mathrm{n}}\right), \\
y_{i} & = & \text { Observed crash count on section } i, \\
\mu_{i} & = & \text { Expected number of crashes on section } i .
\end{array}
$$

Further discussions on the model estimation methodology are provided in Section 4.2.6.

For a given crash severity type, the selection of the negative binomial over the Poisson model for crash modeling is determined by the observed statistical significance of the overdispersion factor. If the overdispersion factor is not significantly different than zero, then the negative binomial model simply reduces to a Poisson regression where the variance equals the mean. If it is greater than zero, then the negative binomial is the correct choice, while the Poisson model is inappropriate. In the present study, both model forms were investigated during the development of the crash prediction models, and the Poisson model was found more appropriate for the fatal crash model while the negative binomial model was found to be superior for analyzing other crash severity types.

\subsubsection{Hypothesis Tests for Significance of the Variables}

For each developed crash prediction model, the statistical significance of each variable was tested at a $90 \%$ level of confidence. This was done to investigate whether any specific variable significantly 
influences crash occurrence at the specified degree of confidence. Because the reported crash frequencies are average values over a stretch of road section, the distribution of crashes was considered as sampling distributions of means, and the population of crash frequencies was assumed to be normally distributed. Taking $\beta_{\mathrm{i}}$ as the estimated coefficient of any crash factor or variable $i$, the hypothesis for testing the significance of that variable was formulated as follows:

$\mathrm{H}_{0}: \beta_{\mathrm{i}}=0$ (variable coefficient is statistically equal to 0 , i.e., variable $i$ is not significant)

$\mathrm{H}_{1}: \beta_{\mathrm{i}} \neq 0$ (variable coefficient is statistically not equal to 0 , i.e., variable $i$ is significant)

This is a two-sided hypothesis test with the "rejection regions" in the lower and upper tails.

Therefore, the critical value of the test statistic is $Z_{\alpha / 2}=Z_{0.05}=1.645$. The calculated value of the test statistic is given by:

$$
\mathrm{Z}^{*}=\left(\mu_{P J}-0\right) /(\sigma / \sqrt{ } n)
$$

Where $\sigma$ is the standard deviation and $n$ is the sample size.

Rejection of the null hypothesis implies that the variable in question is a significant factor in crash occurrence at the specified level of confidence. It is worth noting that in some cases, lack of statistical significance may stem from lack of variability in the values of the variable in question, and in such cases, it should not be construed that the variable is not a significant predictor of crash frequency. For example, for road sections that have the uniform shoulder width, a crash prediction model would indicate that shoulder width is not significant but that does not mean that physical removal of the shoulder would have no effect on crashes.

\subsubsection{Modeling Methodology}

The following steps were adopted for developing crash prediction equations in the present study:

(1) Preliminary descriptive statistical data analysis such as graphs and charts to illuminate general nature of the relationship between crash frequency and the various crash factors (independent variables). These are presented in Chapter 5.

(2) Selection of various alternative mathematical forms to adequately describe the relationship between crashes and crash factors. This involved curve fitting and exploration of various techniques for parameter estimation and model discrimination.

(3) Diagnostic checks to reveal any inadequacies of the developed models and to provide the basis for subsequent model refinement. When the model was found to be adequate according to given criteria [Box et al., 1978], the process was terminated and the resulting model was identified as the "best" model. 
Non-linear models typically require fitting to data via non-linear programming. Due to the nature of the model form, the variable coefficients (or parameters) are not readily obtained using the linear least squares algorithm, and are rather obtained using iterative non-linear least squares methods. Two methods, Least Sum of Squares and Maximum Likelihood, were initially considered for estimating the model coefficients.

\subsubsection{Model Estimation Methods}

\subsubsection{Least Sum of Squares}

In the least sum of squares (LSS) estimation, unknown values of the parameters, $\beta_{1}, \beta_{2}, \beta_{3}, \ldots$ (coefficients of model independent variables), in the regression function $f\left(X_{i}, \beta_{i}\right)$, are estimated by finding the numerical values for the parameters that minimize the sum of the squared deviations between the observed responses and the functional portion of the model. The LSS criterion that is minimized to obtain the parameter estimates is:

$$
Q=\sum_{i=1}^{n}\left[Y_{i}-f\left(X_{i}, \vec{\beta}\right)\right]^{2}
$$

where:

$Y_{i}$, the number of crashes; $\vec{\beta}$ is a vector coefficient; $\beta_{1}, \beta_{2}, \beta_{3} \ldots$ are as coefficients in the optimization process; volume, length, shoulder width, etc., $\left(X_{1}, X_{2}, \ldots\right)$ are variables.

For nonlinear models, the minimization is mostly carried out using iterative numerical algorithms such as the Nelder-Mead Algorithm [Rardin, 2000] and quadratic methods. In the present study, non-linear least squares models were estimated using LIMDEP software in quadratic method [Greene, 2000].

\subsubsection{Maximum Likelihood}

In the maximum likelihood estimation procedure, the log-likelihood function is derived by assuming a distribution for the underlying process. Crash occurrence is random in nature and the occurrence of a subsequent crash is independent of its preceding crash. As such, the crash occurrence process satisfies the characteristics of a Poisson process, and therefore the underlying point process of crashes occurrence on two-lane rural roads may be assumed to be Poisson distributed. The log-likelihood function is given by 


$$
\sum_{i=1}^{n} \text { LogLikelihood }=\sum_{i=1}^{n}\left[-f\left(X_{i}, \vec{\beta}\right)+y_{i} \ln f\left(X_{i}, \vec{\beta}\right)-\ln y_{i} !\right]
$$

Where:

$f\left(X_{i}, \vec{\beta}\right)$ represents the mean of the Poisson distribution for a given set of variables, $\vec{X}$ and $y_{i}$ 's are the incident occurrences (from 1 to $n$ ).

Maximum likelihood estimation involves identifying parameters that maximize the likelihood function for the sample under consideration. In certain cases where the rate of crashes is a random variable rather than a constant, it follows a more general distribution such as gamma distribution. This may be due to the influence of a number of factors on the mean, and could lead to a variance (aggregated by different Poisson means) which exceeds the overall mean. A Poisson process where the mean is gamma distributed follows a negative binomial distribution. The negative binomial model can be used to include variance as an additional variable and relax the mean-variance equality constraint. The general form of the NB model is:

$$
p\left(Y_{i}=y_{i}\right)=\frac{\Gamma\left(y_{i}+\frac{1}{\alpha}\right)}{\Gamma\left(y_{i}+1\right) \Gamma\left(\frac{1}{\alpha}\right)}\left(\frac{1}{1+\alpha \lambda_{i}}\right)^{1 / \alpha}\left(\frac{\alpha \lambda_{i}}{1+\alpha \lambda_{i}}\right)^{y_{i}}
$$

Where:

$\lambda_{i}=E\left(Y_{i}\right)=f\left(\beta_{i}, x_{i}\right), \lambda=f\left(\beta_{i}, x_{i}\right)=$ expected value of incident frequency for the $i^{\text {th }}$ time interval, $\beta_{i}=$ vector of parameters to be estimated, and $x_{i}=$ vector of independent variables.

In the present study, it could not be ascertained that the rate of fatal crashes was randomly distributed, hence a Poisson distribution with constant rates was considered and the parameters were estimated for modeling fatal crashes. Poisson and Negative Binomial models were estimated using the LIMDEP software, a program that estimates and analyzes regression models for qualitative and limited dependent variables [Greene, 1993; Greene, 1995]. The t-statistics were corrected using an over-dispersion parameter. The results of the analysis are presented in Chapter 7.

\subsubsection{Some Modeling Issues}

Prior to the model development, it was necessary to address certain issues that have been identified in past studies as being of enormous importance in crash prediction modeling. These are: 
1. Short Sections vs. Long Sections: What range of lengths of road sections should be used for the analysis? Should the agency use relatively short sections (such as those found in INDOT's current GIS crash database) or relatively long sections (such as those consistent with the following definition: "a portion of road between consecutive intersections or interchanges"). There are pros and cons associated with each choice. In the former case, the advantage is that each individual section is likely to be truly unique in terms of geometric characteristics. However, due to the small size of such sections, they are likely to have relatively few or zero crashes, and such large number of zeros in the database may lead to modeling bias. On the other hand, the use of long sections would minimize the problem of many zeros but could lead to masking of the effect of unique geometric features of individual constituent segments when the several different geometric characteristics are represented by a single average value for the stretch. As a compromise measure, sections defined in INDOT's roadway inventory file were used. These sections have lengths that may be considered as being long enough to obviate the problem of zero inflation, but short enough to ensure maximum consistency of geometric features within each section. The modeling problems associated with the Section Length variable may be further exacerbated in cases where road section lengths are determined using considerations other than intersections, such as change in some crosssectional feature.

2. Aggregation of Crash Data -- Short periods vs. Long periods: Implications of data aggregation were examined not only in the spatial context, but also in the temporal context. The dependent variable in the crash prediction models is crash frequency which may represent the aggregated (total number) of crashes in a given period. The average period may be one day (on one extreme), or several years (on the other extreme), with many possible average periods between these two extremes. There is an inherent danger in using very small lengths of time periods because the crash data would have too many zeros. On the other hand, it is clearly not desirable to aggregate crash frequencies over several years because any changes in the crash factors within such gross temporal aggregations would lead to masking of such effects and thus, bias in the results.

3. Types of Paved and Unpaved Shoulders: In investigating the effects of shoulder paving on crash frequency, what types of shoulder surfaces are considered in (a) the unpaved category (stabilized, gravel, grass, dirt) and (b) the paved category (asphalt, concrete, chip sealed)? In a 
Texas study, the term paved shoulder was defined as "any one of a wide range of all-weather surfaces-bituminous surface-treated shoulder, bituminous aggregate shoulders, full-depth asphalt shoulders, and Portland cement concrete shoulders, ... constructed next to the main line pavements of equal or better type". In other studies, unpaved shoulder was defined as one with gravel, dirt, or grass surfaces free of obstruction for $10 \mathrm{ft}$.

4. Carriageway/Shoulder Delineation: For roadways that have the same material for carriageway and shoulder with no discernible delineation between the two, what criterion is used to determine the existence of a shoulder and its width? For the present study, lane width is defined as the width of the carriageway and was taken/measured as the area (i) between any two marked edge lines or joints on either side of the road, or (ii) between the points where the paved (or graveled) surface ends. For rural two-lane state highways, the shoulder is taken as that provided in the roadway inventory file and for the rural two-lane county roads: any area adjacent to the pavement was considered a shoulder as long as it is driveable (where there were areas with fence walls, cut sections, and embankments in the shoulder area, it was assumed that a shoulder did not exist).

5. Time Series vs. Cross Sectional Modeling: Studies such as the present research that investigate the influence of explanatory factors on a response variable can be carried out in one of two major ways:

The "Before-and-after" Approach: Using data that are spread out over time, the effect of a crash factor can be investigated by comparing crash occurrence at a time when the factor existed and at another time when it did not exist. This method is called a "before-andafter approach". For several sites associated with changes in the levels of that factor over time, the effect of that factor can be investigated using ANOVA techniques. Also, a regression model could be developed to estimate crash occurrence as a function of the change (difference in before and after levels) of the factor. Past research suggests that before-and-after approaches may be prone to a certain type of estimation bias referred to as the regression-to-the-mean effect, and thus may yield unintuitive or unreliable crash occurrence prediction models (unless requisite remedial measures are implemented). Due to this problem and also because of inadequate data on the time trends of safetyrelated road improvement and preservation projects, the before-and-after approach was not used. 
The "Cross Sectional Approach": At any snapshot in time, it is possible to compare the effect of a factor level by estimating the crash reduction between sections that have different levels of that factor. The difference between the factor level effects could be analyzed using ANOVA techniques and a regression model could be developed to estimate crash occurrence as a function of various levels of the factor. The use of these two modeling approaches in developing crash reduction factors are discussed in the next section.

Discussion: In a before-and-after study, the safety effect of a crash factor is determined as the difference in crash frequency at a time when (or at a location where) the crash factor is at a certain level, and the crash frequency at another time when (or at another location where) the crash factor is at another level. In contrast to the before-and-after study, the cross-sectional approach usually employs regression methods to statistically estimate crash frequencies from a large sample of roadway segments whose design attributes vary systematically [Strathman et al., 2001]. The key difference between before-and-after and cross-sectional studies is that the before-and-after study focuses on the changes in safety over time, while the cross-sectional analysis focuses on the differences in safety between locations [Tarko et al., 1998]. The main advantage of the before-and-after approach is that it conforms to the ideal of a controlled experiment. Its main shortcoming is the cost involved in the proper experimental design and execution of such studies, particularly over the range of levels of each crash factor. On the other hand, the main advantages of cross sectional models is that they make use of data that is often readily available at state highway agencies and are much less expensive in terms of time and effort compared to before-and-after studies. The main disadvantage of the cross sectional approach is that it requires an extensive amount of data to ensure proper specification, and is often subject to estimation problems related to data quality. However, gradual automation of roadway inventory data at state level is increasingly mitigating the effect of specification-related problems and is broadening the number and range of crash factors that can be included in cross sectional models. In the present study, cross sectional analysis was used in the development of crash prediction models and subsequently for crash reduction factors (results are shown in Chapter 7).

\subsubsection{Development of Crash Reduction Functions and Factors}

After having established the crash prediction function (also referred to as the safety performance function), the crash reduction factors can be estimated by applying the concepts of differential calculus and elasticities. 
Cross sectional analyses typically involve the use of regression models to estimate the marginal effects of changes in highway design attributes on crash frequencies. One method of obtaining crash reduction factors using this approach is to calculate the elasticity of the parameter estimates from the regression models [Shankar et al. 1997]. The elasticity is defined as the proportionate change in crash frequency resulting from a proportionate change in a given attribute and is obtained by taking the derivative of the crash frequency with respect to a given attribute as follows:

$$
E_{x_{j}}=\frac{\partial \lambda}{\lambda} \times \frac{x_{j}}{\partial x_{j}}=\beta_{j} x_{j}
$$

Where:

$E=$ Elasticity of the $j^{\text {th }}$ independent variable with respect to crash frequency,

$x_{j}=$ Magnitude of the variable under consideration,

$\beta_{j}=$ Estimated parameter for the $j^{\text {th }}$ independent variable,

$\lambda_{i}=$ Expected crash frequency estimated from regression model.

The crash reduction factors calculated in this way are applicable only to small changes in the magnitude of the attribute or when the relationship between crash frequency and the attribute is linear. In an alternative approach that showed that CRF development is an art as much as a science, Tarko et al. [1999] established crash reduction factors associated with a change in a given roadway attribute as follows:

$$
C R F_{x_{j}}=\left(1-e^{\left(\beta_{j} \Delta x_{j}\right)}\right)
$$

Where:

$C R F_{x j}=$ Crash reduction factor associated with the $j^{\text {th }}$ independent variable,

$\Delta x_{j}=$ Change in magnitude of the variable under consideration,

$\beta_{j}=$ Estimated parameter for the $j^{\text {th }}$ independent variable.

The Tarko et al. approach gives nonlinear equations for computing the crash reduction factors and is better suited for predicting the nonlinear change in crashes due to the change in the independent variable. This present study adopts the latter approach to derive the crash reduction factors presented in subsequent sections.

\subsubsection{Categories of Models Developed in Present Study}


To avoid bias inherent in averaging across functional classes and crash types, a family of models was developed in the present study. A single universal model with Functional Class and Crash Type qualitative variables could have been developed. However, in view of correlation problems typically associated with that approach, it was decided to rather have separate models for each functional class and for each crash type - this resulted in a family of models. Table 4-1 shows the various categories of models developed in the present study. Therefore, a total of 13 crash prediction models were developed as part of the present study.

Table 4-1: Categories of Models Developed in Present Study

\begin{tabular}{|l|c|c|c|c|}
\hline $\begin{array}{c}\text { Crash Severity } \\
\text { Functional Class }\end{array}$ & PDO Crashes & Injury Crashes & $\begin{array}{c}\text { Fatal+Injury } \\
\text { Crashes }\end{array}$ & $\begin{array}{c}\text { Total } \\
\text { (All Crashes) }\end{array}$ \\
\hline Rural Collector & $\sqrt{ }$ & $\sqrt{ }$ & $\sqrt{ }$ & $\sqrt{ }$ \\
\hline Rural Minor Arterial & $\sqrt{ }$ & $\sqrt{ }$ & $\sqrt{ }$ & $\sqrt{ }$ \\
\hline Rural Principal Arterial & $\sqrt{ }$ & $\sqrt{ }$ & - & $\sqrt{ }$ \\
\hline County Roads & - & - & - & $\sqrt{ }$ \\
\hline
\end{tabular}

\subsection{FRAMEWORK FOR ENGINEERING NEEDS ASSESSMENT FOR INDIANA'S RURAL TWO-LANE ROADS}

The framework for assessing the physical and monetary needs for addressing engineering safety problems on Indiana's rural two-lane highways was adopted from that developed for INDOT by Lamptey et al. [2004]. This involved establishment of a horizon period, estimation of future crashes at each year within the period (using crash prediction functions in the previous section), identification of hazardous locations, selection of countermeasures and establishing their costs and effectiveness, and optimization to yield deserving sections and associated work needed under various levels of budgetary constraint. The framework is developed for all rural two-lane roads, county or state. In the present study however, the application has been done only for the latter (see Chapter 8).

\subsubsection{Unconstrained Needs Assessment for Deficient Sections at the Rural Two-lane State Highway System}

\subsubsection{Identification of Deficient Locations}


This involved identification of all rural two-lane state highway sections with at least one geometric feature that violates INDOT's specification. Data from the Indiana Safety Management System were used to identify such sections. The Indiana SMS database contains data derived from the roadway inventory file managed and maintained by INDOT's Program Development Division. Generally, there are very little temporal (year-to-year) changes in the physical characteristics of Indiana's rural two-lane roads.

\subsubsection{Physical Needs Assessment on basis of Deficient Sections}

This involved a listing of the various kinds of work needed at "deficient" rural two-lane state highway sections. Physical needs may be expressed in terms of area or linear dimensions. For example, a given section may require shoulder widening by $2000 \mathrm{sq}$. ft, or by $4 \mathrm{ft}$. to each side all along its length. It is worth noting that a deficient section is not necessarily a hazardous section, because some deficient sections have so little traffic that it may not be cost-effective to fix them. Therefore, in a typical network, the set of deficient locations is always much larger than that of hazardous locations, and the funding level needed to address the former always far exceeds that for the latter.

\subsubsection{Monetary Needs Assessment on Basis of Deficient Sections}

This involved estimation of the total funding levels needed to fix all identified physical deficiencies. For each deficiency, the cost of fixing is the unit cost (obtained from literature) multiplied by the extent of the fix needed. For example, the cost of shoulder widening for a given section is estimated as the product of the unit cost of widening (\$ per ft. per mile) and the length of the section.

\subsubsection{Unconstrained and Constrained Needs Assessment for Deficient and Hazardous Sections at the Rural Two-lane State Highway System}

As explained elsewhere in this chapter, a rural two-lane road may be deficient (due to the existing inferior level of engineering feature) but not hazardous (due to a low traffic volume). The previous sub-section shows how the study identified rural two-lane road sections that are only deficient while the present section shows how the study identified road sections that are both deficient and hazardous. The framework for assessing rural two-lane road safety project needs (on the basis of both deficiency and hazardousness) under an unconstrained or constrained budget consists of six analytical procedures that are listed below and subsequently described in detail:

Step 1: Definition of analysis period and network selection 
Step 2: Estimation of expected crash frequency over analysis period

Step 3: Selection of candidate locations over analysis period

Step 4: Identification of alternative safety improvement projects

Step 5: Computation of cost and benefits of safety improvement projects

Step 6: Prioritization to select deserving locations from candidate locations (optimizing investment options under budgetary constraints).

Step 1 - Definition of Analysis Period and Network Selection

The analysis period may be either a long-range planning horizon which typically spans a 20 -year period, or a short-range programming horizon which usually covers a 3- to 5-year period. The network selection involves the definition of a subset of road sections of interest from the entire state road network by attributes such as route type, functional class, county, district or combinations of these attributes.

\section{Step 2 - Estimation of Expected Crash Frequency}

The basic requirement for safety investment is to identify sections within the road network that need some safety intervention at the current or future time. The selection of these candidate locations requires knowledge of the safety performance (crash frequency and severity) of the road network over an analysis period. A considerable amount of research has been conducted on the prediction of expected safety performance of highway segments, as detailed in Chapter 3. Zegeer et al. [1991] developed a non-linear model to predict accidents on horizontal curves. Miaou et al. [1993] used the Poisson model form to predict accidents on road segments. More recently, negative binomial models, a generalized form of the Poisson, have been used in crash modeling. Vogt and Bared [1998] developed the crash prediction models for two-lane rural highways using extended negative binomial regression analysis. The use of the Empirical Bayesian (EB) method in safety analysis has become widely accepted as the most unbiased estimate of the expected crash frequency [Hauer et al., 2002]. It is based on the recognition that historical crash counts are not the only indicator of safety

performance. The EB method also automatically corrects for the regression-to-the-mean effect [Abbess et al., 1981].

For the present study, the crash prediction procedure for the analytical framework is based on the EB method outlined by [Hauer et al., 2002]. The EB estimate uses both historical crash record and expected crash frequency obtained from a multivariate crash prediction model (safety performance function). This is implemented by using a weight factor that depends on the magnitude 
of historical crash record, and the reliability of safety performance functions. For the present study, separate safety performance models were developed for fatal/injury and property damage only using negative binomial analysis of 1997-2000 data extracted from Indiana's crash and roadway inventory databases. Using these models and the observed crash records, the EB estimate of the expected safety performance of a location was then computed as follows:

$$
\begin{aligned}
\varepsilon_{i} & =\omega_{i} a_{i}+\left(1-\omega_{i}\right) x_{i} \\
\omega_{i} & =\frac{1}{1+\frac{a_{i}}{\alpha \cdot L_{i}}}
\end{aligned}
$$

Where

$\begin{array}{lll}\varepsilon_{i} & =\text { EB estimate of crash frequency } \\ \omega_{i} & =\text { Weight factor } \\ a_{i} & = & \text { Expected annual crash frequency on road section } i \text { from safety performance } \\ & \text { function. } \\ x_{i} \quad= & \text { Overdispersion factor of safety performance function } \\ & \text { Number of observed crashes on road section } i\end{array}$

The crash estimates obtained from Equation (4-1) represents the expected crashes for the period where historical crash data is available. To obtain future crash estimates, AADT growth factors were used to convert the expected crash frequency for the before period to an expected crash frequencies for each year of the analysis period.

Step 3 - Selection of Candidate Locations for Safety Improvement

For safety investment, it is sought to select road sections that genuinely require some safety attention now or in the future, and maximizing available resources for a given network over a given analysis period. How does INDOT or LTAP determine which of their deficient rural two-lane roads are actually hazardous? The literature review in Chapter 3 showed how past researchers have addressed this issue. In the present study, the selection of candidate locations strives to combines the best of both methods based on the expected crash frequency and expected crash rate (Lamptey et al., 2004). For instance, the use of the expected crash frequency method results in the selection of locations with the highest potential benefit while the expected crash rate method minimizes the bias of selecting locations with high traffic volume but relatively low crash rate. The method used in the present study may therefore be considered satisfactory from both system and user perspectives. A section is 
selected as a candidate location for safety improvement if both expected crash frequency and crash rate obtained from the EB estimate exceed their respective critical values as shown:

$$
\begin{aligned}
& F_{c(i t)}=D_{a} \cdot L_{i}+k \cdot\left(D_{a} \cdot L_{i}\right)^{\frac{1}{2}} \\
& R_{c(i t)}=R_{a}+k \cdot\left(\frac{R_{a}}{V M T_{i t}}\right)^{\frac{1}{2}}
\end{aligned}
$$

Where

$F_{c(i t)}=$ Threshold or critical crash frequency for road section $i$ in year $t$

$\mathrm{R}_{c(i t)}=$ Threshold or critical crash rate for road section $i$ in year $t$

$D_{a}=$ Average crash density for similar road sections obtained from historical crash records

$\mathrm{R}_{a} \quad=\quad$ Average crash rate for similar road sections obtained from historical crash records

$L_{i}=$ Length of road section $i$

$V M T_{i t}=$ Estimated Vehicle Miles Traveled (VMT) for road section $i$ in year $t$

$k=$ A constant representing the statistical significance of the estimate.

These threshold safety levels indicated above can be updated to reflect current safety goals established by the DOT or local agency. The candidate locations are ranked based on the sum of the ratio of expected and critical crash frequencies and the ratio of the expected and critical crash rates.

\section{Step 4 - Identification of Safety Improvement Projects}

The next step in the analytical framework is to define the set of alternative safety improvements projects to be considered for each candidate location. These improvements vary from site to site and are based on the identification of contributing factors that may be eliminated or changed so that their associated crashes will be reduced or eliminated. Safety improvements projects may be categorized into three main groups based on the contributing factors, namely, vehicle, driver, and the road. In the present study, the framework focuses only on the engineering or road factors. For each candidate location, the factors considered in selecting an appropriate safety project are discussed below.

\section{Deficient Roadway Geometric Features.}

The geometric features considered include right and left shoulder width, lane width, median width, access control, pavement friction, horizontal alignment, and vertical alignment. A roadway feature at a given candidate location is considered deficient if its value at the location is less than the recommended design value obtained from the Indiana Road Design Manual [INDOT, 2000].

\section{Expected Predominant Crash Pattern.}

The crash patterns considered are rear-end, head-on and opposite direction side-swipe, same direction side-swipe, off-road, and night crashes. A crash pattern is identified as predominant if the expected frequency of the particular crash pattern at a given location significantly exceeds the 
critical crash frequency for that particular crash pattern. The framework assumes that the historical proportions of the crash patterns remains unchanged throughout the analysis period. Thus, the expected frequency for the various crash patterns is obtained by distributing the expected crash frequency using default estimates of the historical proportions among the various crash patterns. The critical frequency for each crash pattern is given as:

$$
P_{c(i j)} \quad=\quad P_{a j}+\sigma_{j}
$$

Where

$P_{c(j)}=$ Threshold or critical frequency for crash pattern $j$ for candidate location $i$

$P_{a j}=$ Expected average frequency for crash pattern $j$ for similar road sections

$\sigma_{j}=$ Standard deviation for expected average frequency of crash pattern $j$ for similar road sections

Based on the identified roadway deficiencies and predominant crash pattern, a set of alternative safety improvement projects is identified for each candidate location. For example, a rural two-lane section with predominant off-road crashes is assigned a safety improvement of "Install continuous rumble strips on right shoulder." By default, the "Do Nothing" alternative is added to the set of alternative safety improvement projects for each candidate location. The default set of alternative safety improvement projects for each roadway deficiency and predominant crash pattern (Table 4-2) available in the Indiana Safety Management System Software package (SMS-IN) does not represent the full range of safety improvement projects that can be implemented at a site due to current limitations of data on site specific conditions. The sequence of activities for identifying appropriate countermeasures for the various candidate locations is given in Figure 4-2. 


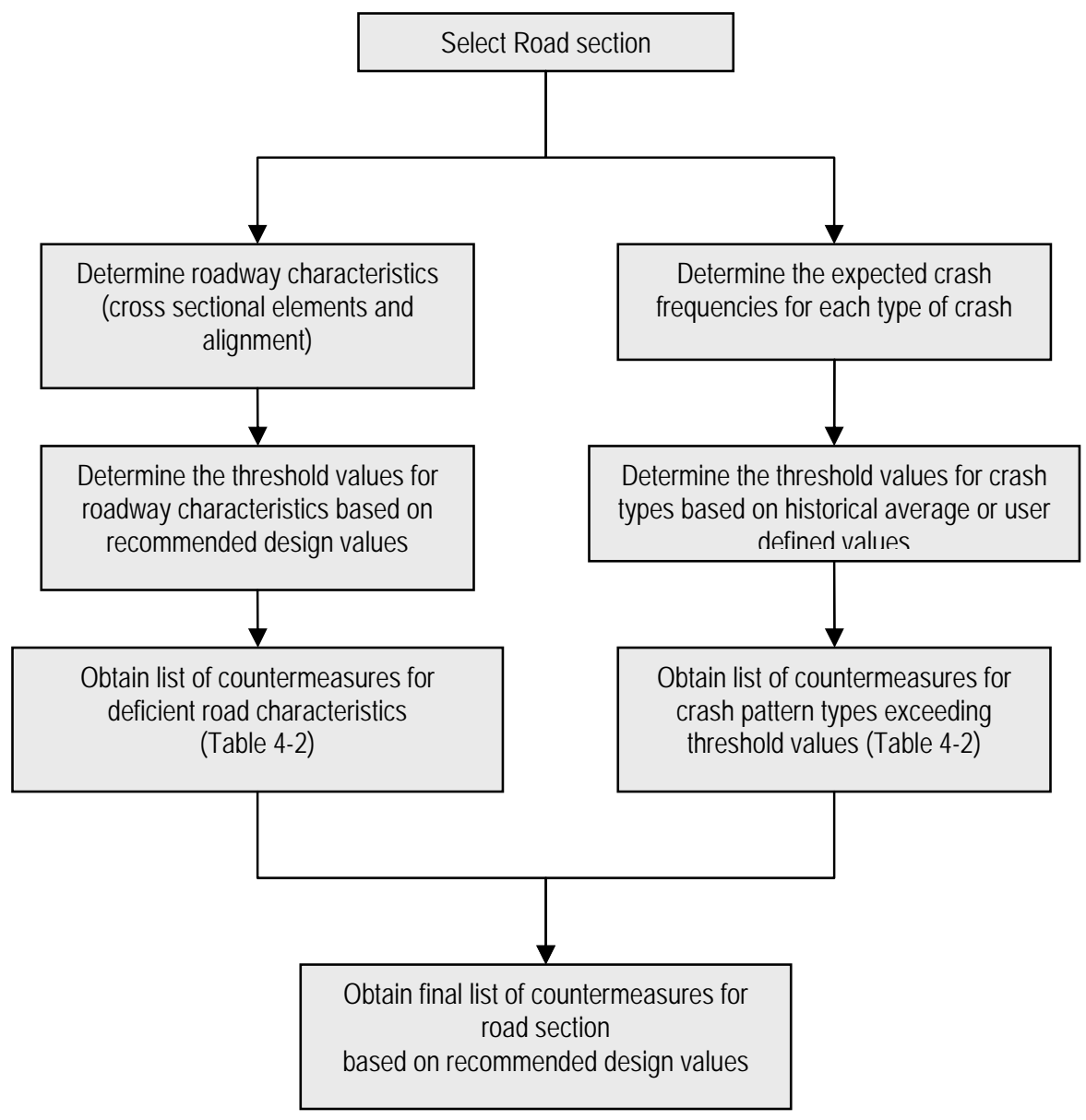

Figure 4-2 Sequence of Activities for Identification of Countermeasures 
Table 4-2 Default Safety Improvement Projects in the SMS Software Package [Lamptey, 2004]

\begin{tabular}{|c|c|c|}
\hline \multicolumn{2}{|c|}{ Road Environment Factor } & Recommended Safety Improvement Project \\
\hline \multirow{7}{*}{ Roadway Deficiency } & Left shoulder width & - Widen left shoulder if less than design standard (2 ft or $4 \mathrm{ft})$ \\
\hline & Right shoulder width & $\begin{array}{l}\text { - Install } 6 \mathrm{ft} \text { right shoulder if not existent } \\
\text { - } \quad \text { Widen right shoulder if less than design standard ( } 2 \mathrm{ft} \text { or } 4 \mathrm{ft})\end{array}$ \\
\hline & Lane width & - Widen roadway lanes if less than design standard ( $1 \mathrm{ft}$ or $2 \mathrm{ft})$ \\
\hline & Median width & - Widen roadway median width if less than design standard \\
\hline & Access control & - Change access control from none to partial control \\
\hline & Horizontal alignment & - Realignment of horizontal curves \\
\hline & Vertical alignment & - $\quad$ Realignment of vertical grades \\
\hline \multirow{5}{*}{$\begin{array}{l}\text { Predominant Crash } \\
\text { Pattern }\end{array}$} & Off road & $\begin{array}{l}\text { - } \quad \text { Install } 6 \mathrm{ft} \text { outside shoulder if not existent } \\
\text { - } \quad \text { Widen right shoulder if less than design standard ( } 2 \mathrm{ft} \text { or } 4 \mathrm{ft}) \\
\text { - } \quad \text { Install guard rail } \\
\text { - } \quad \text { Install rumble strips on outside shoulder }\end{array}$ \\
\hline & $\begin{array}{l}\text { Head on or opposite } \\
\text { direction side-swipe }\end{array}$ & $\begin{array}{l}\text { - Widen roadway lanes if less than design standard ( } 1 \mathrm{ft} \text { or } 2 \mathrm{ft}) \\
\text { - Install non mountable Median for two-lane road } \\
\text { - Install rumble strips on inside shoulder if present }\end{array}$ \\
\hline & Same direction side-swipe & $\begin{array}{l}\text { - Install } 6 \mathrm{ft} \text { right shoulder if not existent } \\
\text { - Widen right shoulder if less than design standard }(2 \mathrm{ft} \text { or } 4 \mathrm{ft}) \\
\text { - } \quad \text { Widen roadway lanes if less than design standard }(1 \mathrm{ft} \text { or } 2 \mathrm{ft})\end{array}$ \\
\hline & Rear end & $\begin{array}{l}\text { - Improve pavement friction if less than design standard } \\
\text { - Install rumble strips in roadway pavement }\end{array}$ \\
\hline & Night Crash & $\begin{array}{l}\text { - Install or improve pavement markings } \\
\text { - } \quad \text { Install or improve roadway lightening }\end{array}$ \\
\hline
\end{tabular}

\section{Step 5 - Computation of Cost and Benefits}

This procedure involves the determination of the costs and benefits associated with the implementation of each alternative safety improvement project over a specified analysis period. Alternative safety improvement projects for a given location can be implemented in any year within the analysis period provided that year equals or exceeds the "critical year" of that location. A deficient road is only considered hazardous if it has sufficient traffic that is exposed to crash likelihood due to the existing defects. As such, the "critical year" for a given location represents the year when the deficient location becomes hazardous due to traffic growth. 


\section{Estimation of Project Costs}

The cost of each safety improvement project is estimated from default unit construction cost, maintenance cost, and salvage cost values which were obtained from Indiana DOT and relevant NCHRP publications. In cases where the service life of the safety improvement project exceeds the analysis period, its value over the remaining service life is taken as a salvage value and discounted to the present year. The present worth of the project costs for each year of the analysis period is estimated as follows:

$$
P W C_{i j t}=\quad C_{i j t} \cdot\left(\frac{1}{(1+r)^{t-1}}\right)+M_{i j t} \cdot\left(\frac{(1+r)^{p-t-1}-1}{r(1+r)^{p}}\right)-S_{i j t} \cdot\left(\frac{1}{(1+r)^{p}}\right)
$$

Where

$$
\begin{aligned}
& P W C_{i j t}=\quad \text { Present worth of costs for safety improvement project } j \text { at location } i \text { at } \\
& \text { analysis year } t \\
& C_{i j t}=\text { Initial construction cost for safety improvement project } j \text { at location } i \text { at } \\
& \text { analysis year } t \\
& M_{i j t}=\text { Annual maintenance cost for safety improvement project } j \text { at location } i \text { at } \\
& \text { analysis year } t \\
& S_{i j t}=\text { Salvage value for safety improvement project } j \text { at location } i \text { at analysis year } t \\
& r=\text { Minimum attractive rate of return } \\
& n \quad=\quad \text { Life span of the safety improvement project } j \\
& t \quad=\quad \text { Analysis year }=1,2 \ldots . . p \\
& p=\quad \text { Analysis period in years }
\end{aligned}
$$

\section{Estimation of project benefits}

The benefits associated with each safety improvement project depend on the expected crash reduction. The crash reduction factors (CRF's) or accident modification factors (AMF's) used in the present study were obtained from a variety of sources such as the Indiana Design Manual [INDOT, 2000], Tarko et al., [1999], Harwood et al. [2003], and Harwood [1993]. The benefits can be computed either in monetary terms (such as the present worth of costassigned crashes) or in non-monetary terms as the number of crashes, fatal crashes, or injury crashes, over the analysis period as follows: 


$$
\begin{aligned}
C R_{i j t} & =\sum_{t}^{p} \sum_{s=1}^{2} N_{s i t} \cdot C R F_{s i j} \\
P W B_{i j t} & =\sum_{t}^{p} \sum_{s=1}^{2}\left(\frac{N_{s i t} \cdot C R F_{s i j} \cdot C C_{s i t}}{(1+r)^{t-1}}\right)
\end{aligned}
$$

Where

$$
\begin{aligned}
& C R_{i j t}=\text { Total Crash reduction for safety improvement project } j \text { at location } i \text { at } \\
& \text { analysis year } t \\
& P W B_{i j t}=\quad \text { Present worth of benefits for safety improvement project } j \text { at location } i \text { at } \\
& \text { analysis year } t \\
& N_{s i t}=\text { Expected crash frequency of severity } s \text { for location } i \text { at analysis year } t \\
& C R F_{s i j}=\quad \text { Crash reduction factor for severity } s \text { associated with safety improvement } \\
& \text { project } j \text { at location } i \\
& C C_{\text {sit }}=\text { Crash cost for severity } s \text { at location } i \text { in analysis year } t \\
& s=\text { Crash severity }(1=\text { Fatal } / \text { Injury crash, } 2=\text { PDO crash }) \\
& r=\text { Minimum attractive rate of return } \\
& n=\text { Service life of the safety improvement project } j \\
& t \quad=\quad \text { Analysis year }=1,2 \ldots . p \\
& p \quad=\quad \text { Analysis period in years }
\end{aligned}
$$

The unit crash costs used for the present worth of benefits computation were updated from the 1994 estimates developed by Indiana Department of Transportation. Either the economic or the comprehensive unit crash costs can be used.

The economic crash cost measures only market costs of the crash (i.e., property damage, medical treatment, and lost productivity, insurance administration and legal costs, and travel delay) while the comprehensive costs takes into account both market and non-market costs (i.e., pain, grief, and reduced quality of life as a result of an injury). For the present study, the unit crash costs were updated from the 1994 dollar estimates developed by INDOT to 2000 dollar estimates using the estimates developed by Blincoe et al. [2002]. Table 4-3 shows the crash cost values used in the present study. 
Table 4-3 Unit Highway Crash Cost Values (2000 dollars)

\begin{tabular}{|l|c|c|c|c|}
\hline \multirow{2}{*}{ Functional Class } & \multicolumn{2}{|c|}{ Economic Costs } & \multicolumn{2}{c|}{ Comprehensive Costs } \\
\cline { 2 - 5 } & Fatal/Injury & PDO & Fatal/Injury & PDO \\
\hline Rural Interstate & $\$ 69,000$ & $\$ 4,600$ & $\$ 153,600$ & $\$ 4,600$ \\
\hline Rural Major Collector & $\$ 78,400$ & $\$ 4,600$ & $\$ 174,300$ & $\$ 4,600$ \\
\hline Rural Minor Arterial & $\$ 78,400$ & $\$ 4,600$ & $\$ 174,300$ & $\$ 4,600$ \\
\hline Rural Other Principal Arterial & $\$ 78,400$ & $\$ 4,600$ & $\$ 174,300$ & $\$ 4,600$ \\
\hline Urban Interstate & $\$ 47,600$ & $\$ 4,600$ & $\$ 105,800$ & $\$ 4,600$ \\
\hline Urban Other Arterial & $\$ 34,500$ & $\$ 4,600$ & $\$ 76,800$ & $\$ 4,600$ \\
\hline Urban Other Freeways and Expressway & $\$ 34,500$ & $\$ 4,600$ & $\$ 76,800$ & $\$ 4,600$ \\
\hline Urban Other Principal Arterial & $\$ 34,500$ & $\$ 4,600$ & $\$ 76,800$ & $\$ 4,600$ \\
\hline
\end{tabular}

Source [INDOT, 2000], [Blincoe et al., 2002]

If a safety improvement project is deferred to a later year in the analysis period the benefits are computed only in terms of the crash reduction between the implementation year and the end of the analysis period. Thus, the penalty for deferring a safety improvement is implicit in Equations 4-10 and 4-11. The current framework does not include other possible benefits from these safety improvements such as travel time savings.

\section{Step 6 - Optimization of investment options}

Highway agencies typically have a budgetary limit for safety improvement projects. There is therefore the need to establish the most suitable safety improvement project and optimal time for implementation at each candidate location within the available budget over the analysis period. Kaji and Sinha [1980] and Pal and Sinha [1998] developed resource allocation methodologies for highway safety improvements using integer programming. Harwood et al. [2003] reviewed various methods of resource allocation such as incremental benefit cost ratio, integer programming and dynamic programming and concluded that when formulated properly, these methods produce similar results. Also, it has been found that integer programming is more efficient than dynamic programming and also simpler than the incremental benefit cost ratio method [Harwood 2003].

For the current study, an integer programming model was chosen based on its identified merits and wide availability of software tools for integer programming. The objective of the optimization is to maximize the total economic value for all the safety improvement projects selected 
for the rural two-lane state highways in Indiana. The economic value $\left(E_{i j t}\right)$ of a safety improvement project $j$ at location $i$ at analysis year $t$ is evaluated using any one of the following alternative economic evaluation criteria:

$\begin{aligned} \text { Cost Effectiveness } & =\frac{C R_{i j t} \cdot 1000}{P W C_{i j t}} \\ \text { Net Present Value } & =P W B_{i j t}-P W C_{i j t} \\ \text { Benefit Cost Ratio } & =\frac{P W B_{i j t}}{P W C_{i j t}}\end{aligned}$

Of the above three methods of economic evaluation, the Net Present Value method was chosen for the network level analysis.

The analytical procedure considers the following scenarios:

1. "Unconstrained funding" needs assessment -- for sections that are both hazardous and deficient.

2. "Constrained funding" needs assessment or "Total Budgeting" optimization -- to determine at which section to do what work, given a budgetary ceiling.

\section{Unconstrained Funding Needs Assessment}

This scenario is consistent with traditional safety needs assessment (in a special case of needs assessment, it is sought to address sections that are both hazardous and deficient sections). There is no budgetary constraint; however only one safety improvement project can be implemented at each candidate location at a time. Strictly speaking, unconstrained needs assessment is not an optimization problem. However, the problem could be set up as one. The funding needs can be determined using the following integer programming equation.

$$
\begin{array}{ll}
\text { Maximize } & \sum_{i}^{h} \sum_{j}^{m} \sum_{t}^{p}\left(x_{i j t} E_{i j t}\right) \\
\text { Subject to } & \sum_{j=1}^{m} \sum_{t=1}^{p} x_{i j t}=1 \text { for all } i \\
& x_{i j t}=0 \text { if } t \neq y_{i} \\
\text { and } & x_{i j t}=0,1
\end{array}
$$


Where

$b \quad=\quad$ Number of candidate locations within selected network

$m=$ Number of alternative safety improvement projects for location $i$

$p \quad=\quad$ Number of years in analysis period

$t \quad=\quad$ Analysis year $=1,2 \ldots . . p$

$y_{i}=$ Year when location $i$ becomes hazardous (critical year)

$E_{i j t}=$ Economic value of safety improvement project $j$ at location $i$ in analysis year $t$

$x_{i j t}=\left\{\begin{array}{l}1 \text { if safety improvement project } j \text { is implemented at location } i \text { for analysis year } t \\ 0 \text { otherwise }\end{array}\right.$

Equation (4-18) seeks to maximize the total economic value of the selected safety improvement projects. The economic value $E_{i j t}$ for each alternative safety improvement project at each candidate location is determined from Equation (4-15), (4-16), or (4-17). The constraints on the optimal solution are represented by Equations (4-19) to (4-21).

\section{Total Budgeting Constrained Optimization}

"Total budgeting" represents the situation where a given budget is specified for the entire analysis period and there are no constraints as to the amount that can be spent in a particular year. For this scenario, the constraint is the total funding available for the entire analysis period. The optimal allocation of the funding can be obtained by solving the following integer programming equation:

$$
\begin{array}{ll}
\text { Maximize } & \sum_{i}^{h} \sum_{j}^{m} \sum_{t}^{p}\left(x_{i j t} E_{i j t}\right) \\
\text { Subject to } \quad & \sum_{i=1}^{h} \sum_{j=1}^{m} \sum_{t=1}^{p}\left(x_{i j t} C_{i j t}+(p-t) x_{i j t} M_{i j t}\right) \leq B \\
& \sum_{j=1}^{m} \sum_{t=1}^{p} x_{i j t}=1 \text { for all } i \\
& \sum_{i=1}^{h} \sum_{j=1}^{m} x_{i j t} \geq 1 \text { for all } t \\
& x_{i j t}=0 \text { if } t<y_{i} \\
& x_{i j t}=0,1
\end{array}
$$


Where

$M_{i j t} \quad=$ Annual maintenance cost of safety improvement project $j$ at location $i$ for analysis year $t$

$C_{i j t}=$ Initial capital cost of safety improvement project $j$ at location $i$ for analysis year $t$

$B \quad=$ Total budget for analysis period.

Other symbols have their usual meanings.

Equation (4-22) represents the objective function of the integer program, similar to Equation (4-18). The constraints on the optimal solution are represented by Equations (4-23) through (4-27). Equation (4-23) constrains the total expenditure (initial capital and annual maintenance cost) by the budgetary ceiling over the analysis period. Equation (4-24) requires that only one safety improvement project (including the Do-nothing alternative) should be selected for each candidate location while Equation (4-25) requires that at least one safety improvement project (including the Do-nothing Project) should be implemented in each year of the analysis period.

For any of the above integer programs, the optimal solution is defined as the set of safety improvement projects and their respective locations and implementation years that provides the maximum economic value subject to the given constraints. The implementation schedule for the safety improvement projects at the rural two-lane state highway sections is then prioritized on the basis of the implementation year and the critical value obtained from the procedure where candidate locations were selected. For example, if two safety improvement projects are to be implemented in the same year, then that with the higher critical value is given a higher priority for implementation.

\subsection{CHAPTER SUMMARY}

This chapter discussed the overall framework, methodologies, and underlying theories that were used in addressing the study objectives. The framework covered the development of crash prediction equation and crash reduction factors, and network-level needs assessments. The methodologies presented were specifically chosen for the analysis in view of the findings of the literature review and also to help facilitate reliable data analysis and interpretation that is vital for studies of this nature. 


\section{CHAPTER 5 DATA COLLECTION AND COLLATION}

\subsection{DATA SOURCES FOR RURAL TWO-LANE STATE ROADS}

In order to demonstrate the application of the study methodology (described in the previous chapter) to address the study objectives (stated in Chapter 1), databases were developed separately for the state highways and county roads. For the state highways, a the database was developed from a number of separate datasets - the Roadway Inventory Dataset, Route Alignment Dataset, Indiana Crash Dataset, and the Road Sections Dataset.

The sections below discuss the details of each dataset. In each dataset, road segments are identified using a unique code specifically designed for that dataset. As such, considerable effort was expended in linking the datasets so that all data types relating to a particular segment could be brought to a common reference. Figure 5-1 illustrates the relationships between the various components of the database.

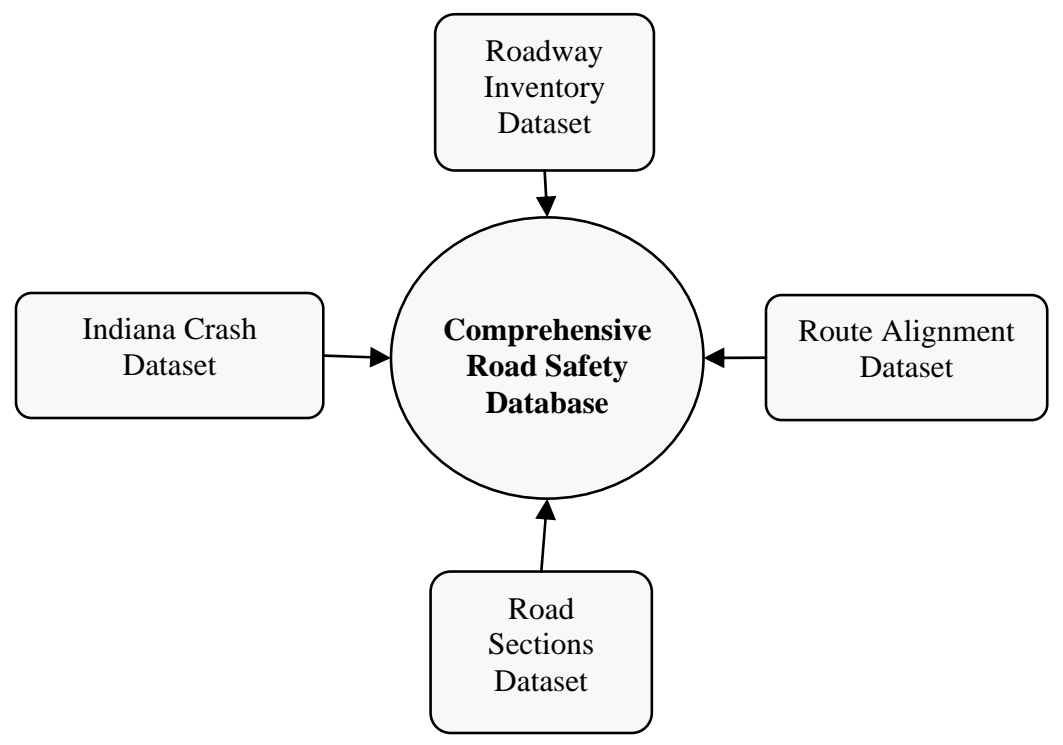

Figure 5-1 Components of the Comprehensive Road Safety Database 


\subsubsection{The Roadway Inventory Dataset}

The roadway inventory dataset for the rural two-lane state highways contains 50 fields which provide detailed information on the location, cross sectional elements, traffic volume and pavement condition for all road segments on the state highway network. Each segment in the inventory database is identified by a unique segment number, i.e., a particular route may consist of different homogeneous sections each of which is represented by a unique segment number. The roadway inventory dataset was supplied by INDOT’s Program Development Division.

\subsubsection{The Route Alignment Dataset}

The route alignment dataset, obtained from INDOT, was developed as part of efforts by another JTRP research project that is developing a complete HPMS database compatible with the Highway Economics Requirements System. The dataset contains information on the alignment characteristics of roads on the Indiana road network. The alignment dataset consists of separate files for horizontal and vertical alignments. The horizontal alignment file contains information such as the length, radius and degree of curvature for each horizontal curve while the vertical alignment file contains information such as the length, start and end grades and $K$-values (which reflect degree of curvature) for each vertical curve. The chainage (milepost location) of each curve on the route is also provided.

\subsubsection{The Indiana Crash Dataset}

Maintained by the Center for Advanced Transportation Safety (CATS), the Indiana crash database served as the source for crash data. This dataset contains 44 fields which provide comprehensive information of each crash recorded on the Indiana road network. Each record in the dataset represents a crash $\log$ which provides information such as the crash location (route, distance from nearest intersection, township or city), crash severity and pattern, and the primary cause of the crash. The crash database is based on data received annually from the Indiana State Police. For the present study, crash data for 1997 to 2000 were used.

\subsubsection{Road Sections Dataset}

The road sections dataset was developed from a spatial integration of layers from INDOT's GIS map layers with the road inventory dataset, signalized intersection dataset, township boundaries, metropolitan areas, and county boundaries. This was done to generate a unique field identifier among the various datasets and consequently, to facilitate their merging. Each record in this dataset comprises of the route name, the start and end chainages defined by township or city boundaries, the 
township, county and district. The decision to use the township and city boundaries as the start and end chainages for the road section is due to the fact that the location of each crash occurrence in the crash database is identified with a pair of pseudo numbers - one for the main street and one for the cross street. However, it was found that there is no completely reliable way to assign a crash occurrence to a road segment in the road inventory dataset due to the following problems:

1. Inconsistent use of both local and state names for road identification resulting making it difficult to identify some pseudo numbers of the Indiana road inventory database.

2. The pseudo numbers for either the main street or the crossing street were not coded for some sections, which led to a number of crashes not being identified.

3. Existence of duplicate pseudo numbers i.e., two sections having the same pair of pseudo numbers making it impossible to distinguish between these sections.

In the crash database, individual crashes can be grouped by route name, county and township or city. As such, knowledge of such locational characteristics, together with the start and end reference points of the road section, facilitated integration of the various datasets. This method of integrating the various datasets overcomes the difficulties mentioned above. A visual basic program code, the conceptual algorithm for which is presented in Figure 5-2, was then developed to link the datasets. The route sections within small townships are categorized as rural roads, while those within the metropolitan areas/cities are categorized as urban roads and were therefore excluded from the present study. Certain road sections in the crash database could not be identified in the road inventory database probably due to an error in coding or change in road name. Where this situation occurred, the "offending" record (road section) was expunged from the modeling dataset. 


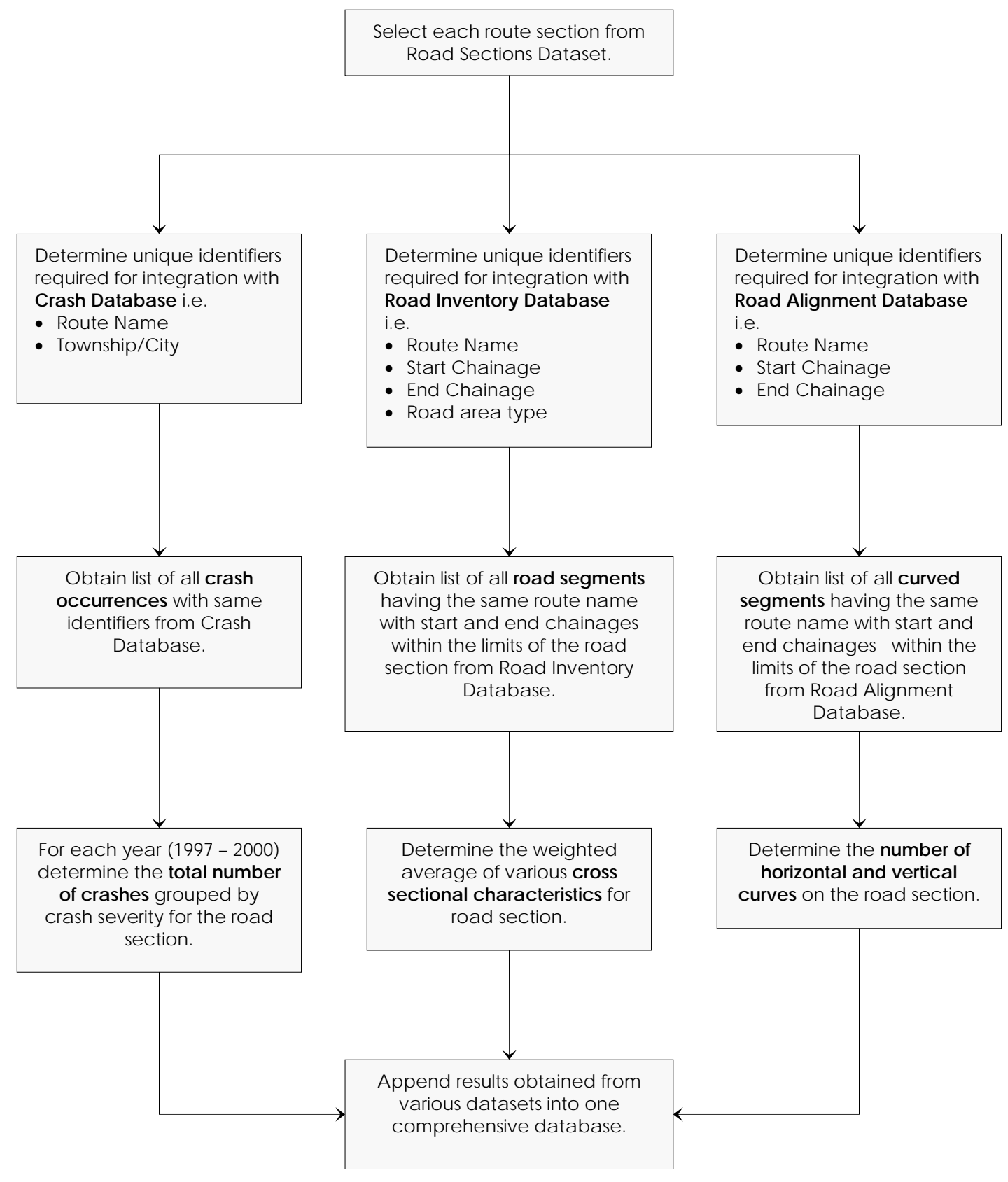

Figure 5-2 Concepts of Algorithm Used for Linking Data from the Various Datasets

The comprehensive database contains 49 variables providing most of the information about the road section required for the model development. Table 5-1 presents the categories and variable codes in the modeling dataset. This dataset provided the platform required for the integration of the various datasets. Figure 5-3 shows a map of the Indiana state highway network. 
Table 5-1: Variables in the Overall Modeling Dataset

\begin{tabular}{|c|c|c|c|}
\hline Category & $\#$ & Variable & Description \\
\hline \multirow{10}{*}{ Route Identification } & 1 & ID & Record ID \\
\hline & 2 & ROUTE_NAME & Route Name \\
\hline & 3 & AREA & Route Area Type (Rural or Urban) \\
\hline & 4 & FC & Functional Class \\
\hline & 5 & $\mathrm{CO}$ & County \\
\hline & 6 & DIST & District \\
\hline & 7 & TWP & Township \\
\hline & 8 & FROM & Start Chainage \\
\hline & 9 & $\mathrm{TO}$ & End Chainage \\
\hline & 10 & LENGTH & Length of section (to the nearest 0.01 mile) \\
\hline \multirow{20}{*}{ Crash Data } & 11 & FAT97 & Fatal Crashes on road section for 1997 \\
\hline & 12 & INJ97 & Injury Crashes on road section for 1997 \\
\hline & 13 & PDO97 & PDO Crashes on road section for 1997 \\
\hline & 14 & FATINJ97 & Fatal/ injury Crashes on road section for 1997 \\
\hline & 15 & TOTAL97 & Total Crashes on road section for 1997 \\
\hline & 16 & FAT98 & Fatal Crashes on road section for 1998 \\
\hline & 17 & INJ98 & Injury Crashes on road section for 1998 \\
\hline & 18 & PDO98 & PDO Crashes on road section for 1998 \\
\hline & 19 & FATINJ98 & Fatal/ injury Crashes on road section for 1998 \\
\hline & 20 & TOTAL98 & Total Crashes on road section for 1998 \\
\hline & 21 & FAT99 & Fatal Crashes on road section for 1999 \\
\hline & 22 & INJ99 & Injury Crashes on road section for 1999 \\
\hline & 23 & PDO99 & PDO Crashes on road section for 1999 \\
\hline & 24 & FATINJ99 & Fatal/ injury Crashes on road section for 1999 \\
\hline & 25 & TOTAL99 & Total Crashes on road section for 1999 \\
\hline & 26 & FAT00 & Fatal Crashes on road section for 2000 \\
\hline & 27 & INJ00 & Injury Crashes on road section for 2000 \\
\hline & 28 & PDO00 & PDO Crashes on road section for 2000 \\
\hline & 29 & FATINJ00 & Fatal/ injury Crashes on road section for 2000 \\
\hline & 30 & TOTAL00 & Total Crashes on road section for 2000 \\
\hline \multirow{17}{*}{$\begin{array}{l}\text { Cross sectional } \\
\text { characteristics }\end{array}$} & 31 & $\mathrm{LN}$ & Number of lanes \\
\hline & 32 & AADT97 & AADT for 1997 \\
\hline & 33 & AADT98 & AADT for 1998 \\
\hline & 34 & AADT99 & AADT for 1999 \\
\hline & 35 & AADT00 & AADT for 2000 \\
\hline & 36 & LW & Lane width \\
\hline & 37 & MW & Median width \\
\hline & 38 & SW & Left Shoulder width \\
\hline & 39 & LSW & Right shoulder width \\
\hline & 40 & ST & Shoulder type \\
\hline & 41 & FR & Pavement friction \\
\hline & 42 & SI & Pavement serviceability index \\
\hline & 43 & SR & Pavement serviceability rating \\
\hline & 44 & $\mathrm{AC}$ & Access control \\
\hline & 45 & PRK & Presence of parking \\
\hline & 46 & $\mathrm{CRB}$ & Presence of curbs \\
\hline & 47 & $\mathrm{AL}$ & Presence of turn lanes \\
\hline \multirow{2}{*}{ Alignment } & 48 & NHCUR & Number of horizontal alignments \\
\hline & 49 & NVCUR & Number of vertical alignments \\
\hline
\end{tabular}




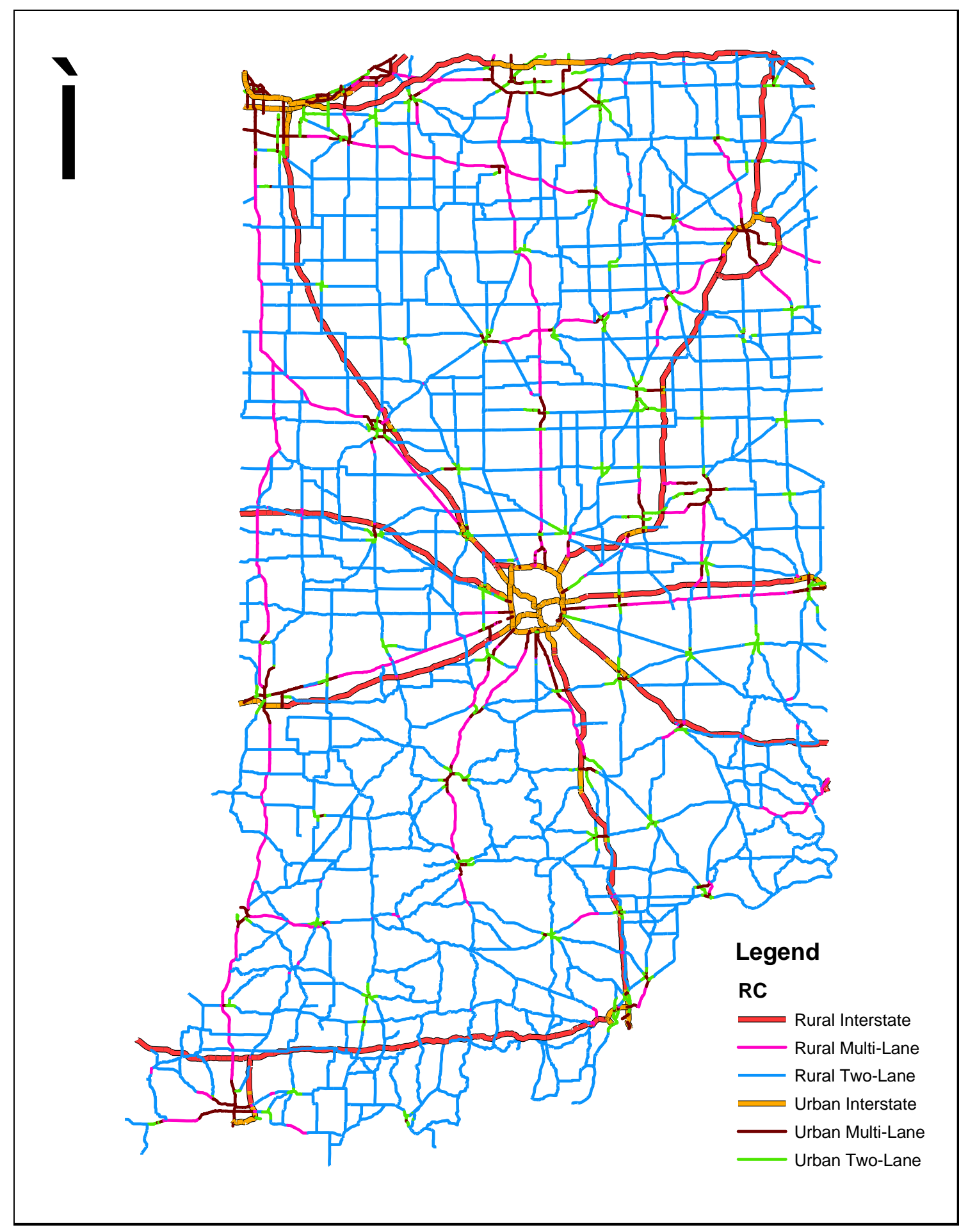

Figure 5-3 The Indiana State Highway Network 


\subsection{DATA SOURCES FOR RURAL TWO-LANE COUNTY ROADS}

Due to lack of county road crash history data at a statewide level, only a sample was used. Sources for county roads data included field surveys by the Research Team (for inventory data), and the Indiana LTAP office and the Tippecanoe county officials (for crash history data).

\subsubsection{Data Set Description}

\subsubsection{Crash data base}

The crash data base for seven different years (1995-2001) was collected from the county's safety engineer. The crash database contained descriptions on the following:

- Crash Identification Number

- Date of Crash

- Time of Crash

- Location of Crash

- Number Injured in Crash

- Number of Vehicles Involved in Crash

- Crash severity

- Drivers Physical Condition

- Light and Weather conditions

- Collision Type

- Distance from the Intersection

\subsubsection{Road Characteristics Database}

A county road inventory database was also collected from the Tippecanoe county safety engineer. This database consisted of the following characteristics:

- Name of Township

- AADT

- Roadway Segment Length

- Surface Type

- Roadway Segment name

- Roadway Segment Section Number

The road section names and referencing codes were used to integrate the data from the crash and road inventory databases. 


\subsubsection{Field Roadway Inventory}

The roadway inventory files described above did not contain adequate information on the geometric characteristics of the County's roadway segments. As such, a field inventory of a random sample of 107 county road segments in Tippecanoe county was carried out as part of the present study (Plate 51). These road segments represented 6 of the 12 townships in the county. Data was collected for each segment, and a "segment" was defined as the stretch of road between two successive intersections. Data collected in the field survey included lane width, shoulder width, shoulder type, driveway density, posted speed limit, number of horizontal curves, and pavement marking. The number of vertical curves was not included as most of the roads generally had flat terrain. In general, all road sections that were inventoried had the same characteristics for both directions. For example, for each segment, the shoulder type and width on the left hand lane was same as that on the right hand lane.

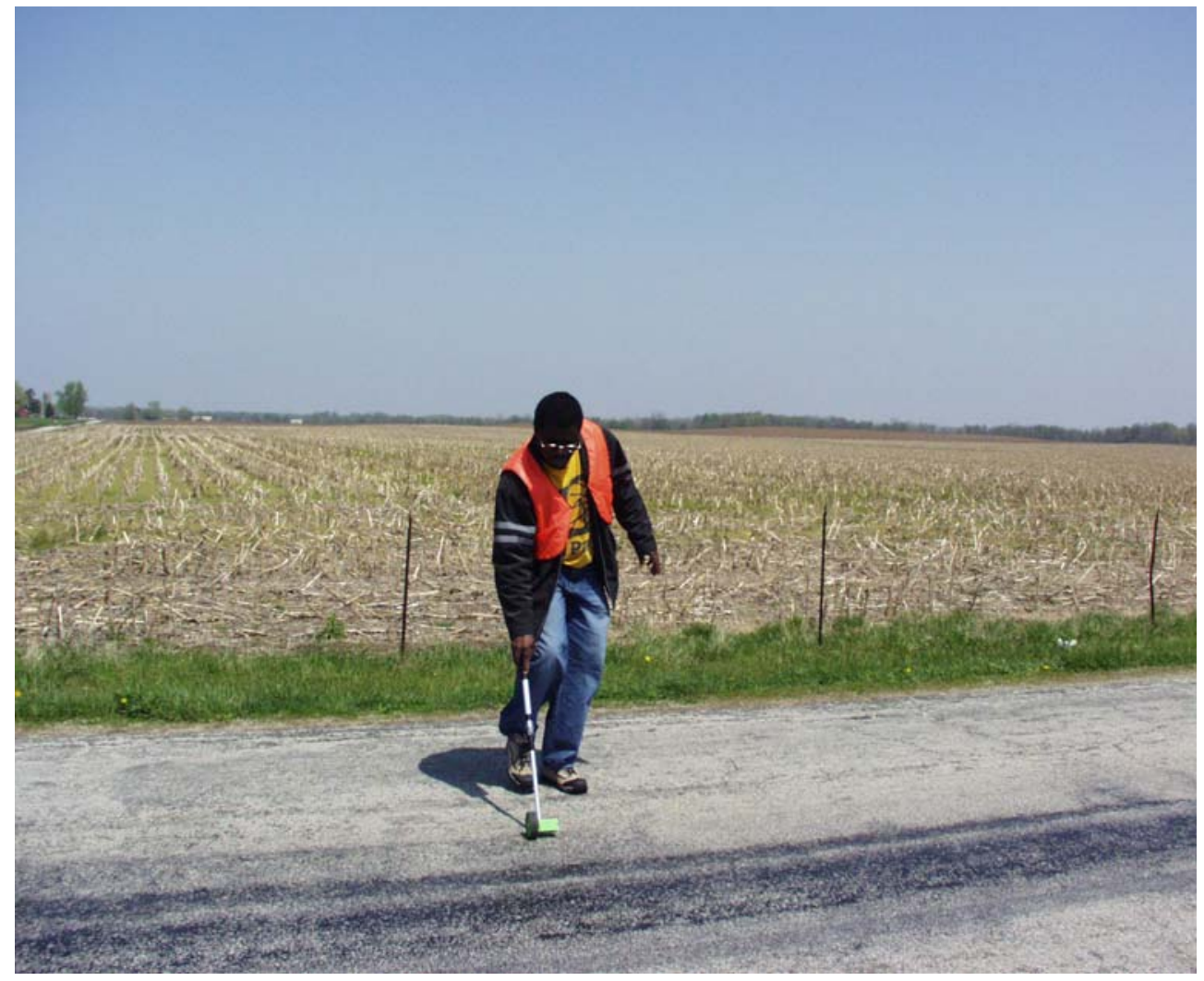

Plate 5-1: County Road Data Collection by the Research Team, April 2003 


\subsubsection{Data Processing}

The data obtained from the field inventory were processed in a form that was useful for modeling purposes. For example, all the 107 roadway segments had different lengths. As such, driveway density instead of driveway frequency was used for the modeling. Driveway density is simply the segment length divided by the number of driveways.

\subsubsection{Data Extraction}

\subsubsection{Extraction and Merging of Traffic Volume Data}

Due to differences in the formatting of the road section names in the road section database and the crash database, the two databases could not be simply merged. After field data had been collected for all 107 segments, the existing road inventory data for these segments were manually extracted and integrated with the collected field data to form a new inventory database solely for these sections.

\subsubsection{Extraction and Merging of Crash Data}

After the crash data for the three years had been processed by year, the data were merged with the integrated road inventory database. This was done using the COUNTIF function in Microsoft Excel. This macro extracts the total number of crashes for each road segment using the road section number, which matched those in the integrated road inventory database. The completion of this process yielded the final datafile for the modeling process.

\subsubsection{Problems Encountered with Data Collation}

Problems included missing data in the road inventory and crash databases. These databases had a few missing field names and entries. There was also the problem of crash descriptions of unknown codes. These problems were subsequently addressed by obtaining a copy of the Indiana officer's standard accident report. Another problem involved the merging of the crash and road inventory data. As discussed in the previous section, this could not be done by code because the road name (a field that was common to both source datasets) had been formatted differently in the datasets. Also, the road segment number was available in only some of the provided crash data sets. As such, such merging was done manually. Yet another problem involved the determination of which crashes actually occurred on the roadway segments, and not at nearby intersections. To address this problem, a benchmark value of 50 feet (for county roads) was used to differentiate road section crashes from intersection crashes. 
In what was probably the most significant problem associated with the county roads data collection, there seemed to be a rather large discrepancy in a few reported AADTs. Furthermore, there were some segments with missing AADT values. Such sections were expunged from the modeling dataset. 


\section{CHAPTER 6 DESCRIPTION AND DISTRIBUTION OF EXPLANATORY CRASH FACTORS}

As mentioned in Chapter 1 of this report, crash experience is the culmination of a gamut of factors that include engineering (roadway) features, vehicle and operator characteristics, the environment, and levels of education and enforcement of legislation geared towards safe roadway use. The present study focuses only on the engineering features of the roadway. As illustrated in Figure 61, such engineering features may generally be placed in six categories: Sectional Characteristics, Nature of the Alignments, Roadway (Carriageway) Features, Nature of the Shoulder, Roadside Features, and Traffic Control Devices.

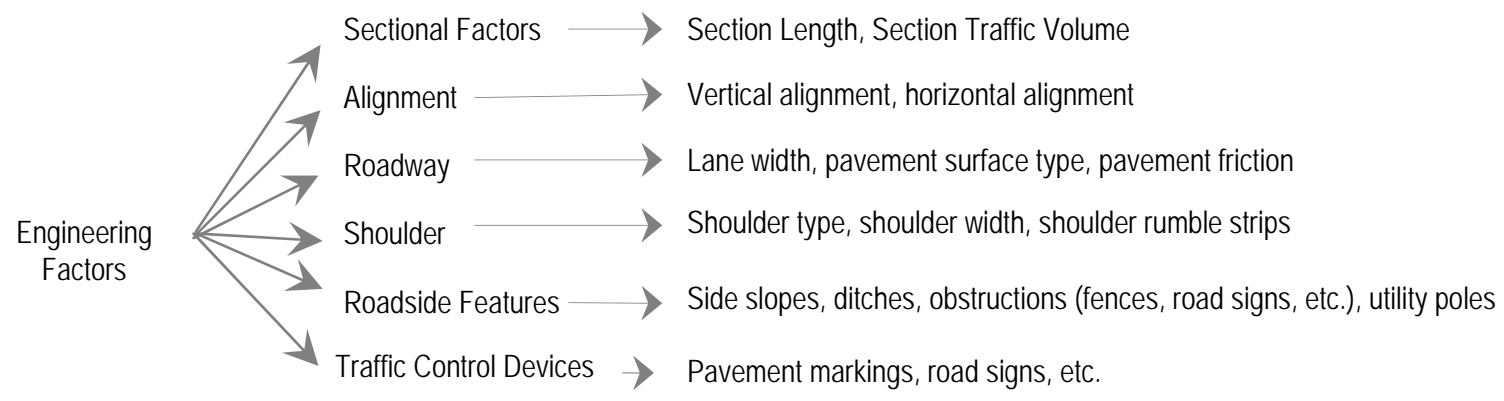

Figure 6-1: Explanatory Engineering Factors Affecting Crashes on Rural Two-lane Roads

For each of the above factors, this chapter presents:

- a description of the defects or situations associated with the factor,

- possible countermeasures to address the problem,

- demonstrated effectiveness of such countermeasures (from past studies), and

- the extent of problems associated with that factor, for rural two-lanes roads in Indiana. This includes a description of the magnitude of the problem and a preliminary descriptive assessment of the effect of that factor on crash rates using Indiana data. Tools used include binned bar charts and correlation coefficients. A positive correlation coefficient indicates that as that engineering factor increases, crash rates also increases; while a negative coefficient indicates the contrary. 


\subsection{CARRIAGEWAY FACTORS}

A key element of highway geometric design is the selection of a horizontal and vertical alignment, and cross sectional types and dimensions that satisfy the constraints of the existing topography as well as the expected traffic. Horizontal and vertical alignment considerations include the degree and lengths of curves and grades of vertically straight sections. Cross sectional features of the carriageway include lane-width, pavement type, surface friction, cross slopes, superelevation, etc. In its publication titled "A policy on Geometric Design of Highways and Streets" (also known as the Green Book), AASHTO established recommended standards for roadway geometry on the basis of road functional classification [AASHTO, 1994].

\subsubsection{Carriageway Lanes}

Carriageway lanes (also often referred to as "travel lanes") are the part of the roadway that is intended for use by vehicular traffic. The lane width of a two-lane road is measured as the distance between the centerline of the roadway to the pavement edge. Lanes of adequate width increase the opportunity for recovery of errant vehicles and also provide increased lateral separation between passing and opposing vehicles. Several studies have been carried out to relate lane width to crash experience [Zegeer et al., 1987; Tarko et al., 1997; Harwood et al. 1998]. Fitzpatrick et al. [2000] argued that there is a need to express crash experience as a function of crash patterns because lane width logically affects certain crash patterns (such as run-off-road, head-on, and sideswipes) but not necessarily other crash patterns (such as angle and rear end crashes).

A natural countermeasure to address the problem of inadequate lane width is to provide more width for a driver to recover if the vehicle begins to leave the carriageway, and also to increase the separation distance between opposing traffic streams. Several studies have found that increasing the lane width is associated with reduced crashes. Using data for over 5,000 miles of two-lane road segments from seven states, a 1987 FHWA study investigated the effects of lane width on highway crash experience, duly controlling for roadway, terrain, and traffic features [Zegeer et al., 1987]. That study determined that accident types related to lane width included run-off-the-road, head-on, samedirection-sideswipe, and opposite-direction-sideswipe (herein referred to as "related" crashes). That study also estimated the percent reduction in crash frequency as a result of increasing lane widths (Table 6-1). It is seen that significant reduction in lane frequency may be obtained by increasing the lane width. For example, widening a lane by $4 \mathrm{ft}$ could yield as much as $40 \%$ reduction in crashes. 
Table 6-1: Effectiveness of Lane Widening with Respect to "Related "Crashes

\begin{tabular}{|c|c|}
\hline Amount of Lane Widening (ft) & \% Reduction in Crashes \\
\hline 1 & 12 \\
\hline 2 & 23 \\
\hline 3 & 32 \\
\hline 4 & 40 \\
\hline
\end{tabular}

Note: These values are only for rural two-lane roads.

Source: [Zegeer et al., 1987]

Zegeer and Deacon (1987) analyzed data covering a wide range of lane-widths and provided a critique of over 30 years of literature to summarize safety relationships between lane-width and crashes. Among their conclusions was that lane conditions have a greater effect on "run off the road (ROR)" and "other direction (OD)" crashes compared to rear-end and angle crashes. Such crashes decrease with increasing shoulder width and lane width. It was observed that compared to shoulder width, lane width has larger impact on crash rates within the range of shoulder and lane widths studied.

Abdel and Radwan (2000) estimated a negative binomial regression model for an arterial in Florida and concluded that improvements in horizontal curvature, and shoulder, lane and median widths, reduce crashes. Also, in a study for rural two-lane roads, Vogt and Bared (1998) evaluated changes in design parameters using data from Highway Safety Information System. Using a population of highway segments from Washington and Minnesota they derived statistical models linking design elements to crashes and determined that increasing lane widths and lesser curvatures led to decreased fatalities.

In a study that investigated the interrelated effects of various combinations of lane and shoulder widths, Zegeer et al. [1981] found that of the various crash types, only ROR and OD crashes were significantly influenced by lane and shoulder width. They found that rates of these two crash types generally decrease as lane and shoulder widths increase, but only up to a point (11-12 ft), after which further increases in lane widths are ineffectual (Figure 6-2). Commenting on the Zegeer et al. study, Hauer [2000] argued that the effect of shoulder widening on all crashes, rather than only ROR and OD crashes, should be the focus. 


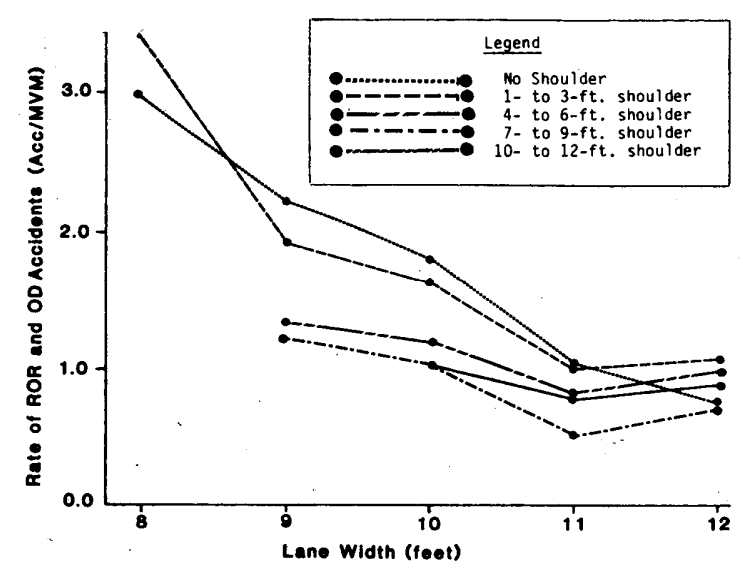

Figure 6-2: Relationship between Lane Width and Crashes [Zegeer et al., 1981]

In an NCHRP study that utilized data from several states, Zegeer et al. [1994] found that lowvolume roads experience a slightly higher percentage of injury crashes than all rural roads. The researchers determined that single vehicle crashes (fixed object, rollover, run-off-road) are proportionately more frequent, and multi-vehicle crashes (rear end, angle, turning) are proportionately less frequent on low volume roads than all rural two-lane roads. Also, the presence of a shoulder was found to be associated with significant crash reductions particularly where lane widths were at least 10 $\mathrm{ft}$. The study produced evidence that $9 \mathrm{ft}$ lanes with wide shoulders may be preferable to $10 \mathrm{ft}$ lanes with narrow shoulders, and that crash experience does not appear significantly different for unpaved versus paved roadway surfaces at traffic volume levels of $250 \mathrm{vpd}$ or less - above this volume, crash rates are significantly greater for unpaved roads. That finding provided supportive evidence for paving roadways with traffic volumes above 250 ADT for safety reasons. The study found that crash rates generally increase with increasing combined (roadway plus shoulder) width up to $28 \mathrm{ft}$, and then decreases with increasing combined width thereafter (Figure 6-3).

It is therefore clear that results from previous studies are suggestive of a "humped" relationship between lane (and shoulder width) and crashes. There seems to be an initial direct relationship (increase in crashes with increased width), but only for a short range of widths, after which there is a general decreasing crash occurrence with increasing width. But how far does this inverse relationship extend? Is there a limit to the benefits of increasing lane width? The answer seems to be affirmative, for certain studies have explicitly found that increasing lane width generally reduces crashes, but only up to a point, after which increasing lane width results in increasing crashes. 


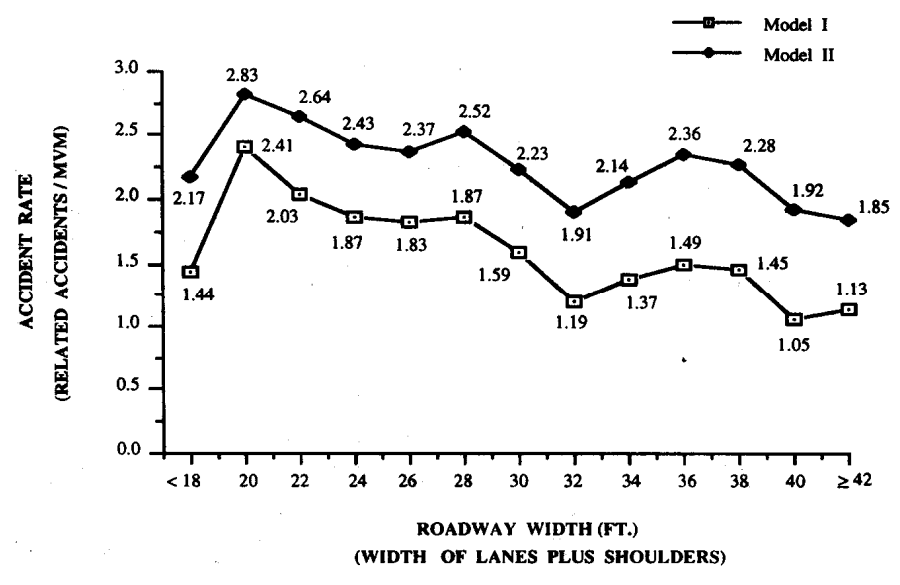

Figure 6-3: Relationship between Combined Width and Crashes [Zegeer et al., 1994]

This seems to be quite intuitive. From a psychological point of view: when lane width exceeds a certain point, drivers may become careless or may become uncertain about the exact position they should occupy on the carriageway, and such uncertainty could confuse following vehicles, leading to traffic conflicts and consequently, crashes. Furthermore, while increasing lane widths may generally be considered effective in reducing crashes, such treatment may not necessarily be cost-effective. In other words, the cost of lane widening may sometimes outweigh the benefits it yields. Griffin and Mak [1989] established that roadway widening was generally not cost-effective for two-lane farm-to-market roads with ADTs below 1,000 vehicles per day. As most of Indiana's county roads seem to fit the physical and operational description of such roads, it would be interesting to investigate the effectiveness of lane and shoulder widening on Indiana's county roads. This is addressed in the present study.

\subsubsection{Distribution of Carriageway Lane Widths at Rural Two-lane Highways in Indiana, by Functional Class}

From the dataset prepared for the present study, the distribution of carriageway lane widths at Indiana's rural two-lane highways was investigated. This was done for both functional class (Figure 6-4) and region (Figure 6-5). The lane width for each road section is the average of the northbound and southbound travel lane. For sections comprised of segments with different lane widths, the weighted average of the constituent segments were found and used to represent the lane width of the section. For purposes of the preliminary descriptive analysis, the following ranges were used for categorizing the lane widths: 


$$
\begin{aligned}
\text { Very } & 8 \leq L W<11 \mathrm{ft} \\
\text { Small: } & \quad 11 \leq L W<12 \mathrm{ft} \\
\text { Small: } & 1 \leq \mathrm{ft} \\
\text { Medium: } & L W=12 \mathrm{ft} \\
\text { Large: } & L W>12 \mathrm{ft}
\end{aligned}
$$

The preliminary descriptive analysis showed that the carriageway width generally ranges from 8 to $15 \mathrm{ft}$ for rural major collectors and from 9 to $15 \mathrm{ft}$ for rural minor and principal arterials. Approximately $40 \%$ of rural major collector lanes exceed $11 \mathrm{ft}$, while approximately $98 \%$ of rural principal arterial lanes exceed $11 \mathrm{ft}$. The case for rural minor arterials lies in between these two, and has an approximately normal distribution centered around $11.5 \mathrm{ft}$. This is evidential of the relatively higher geometric standards that exist for rural principal arterials and the relatively lower standards for rural major collectors.

For purposes of engineering studies, the state of Indiana is often divided into three regions: northern (Laporte and Fort Wayne Highway Districts), central (Crawfordsville and Greenfield Districts), and southern (Vincennes and Seymour Districts). It is possible that there may be regional variations in geometric features due to differences in management and administrative policies and practices. The regional distribution of the carriageway widths of roads showed relatively little variation (Figure 6-5). However, it was found that the lane widths are approximately normally distributed for each region, with mean, median and modal values ranging between 11 and $12 \mathrm{ft}$. There is obviously no statistically significant difference in the distribution of lane widths across the three regions in Indiana. As such, there may be no need to consider regional locations of roads in investigating the impacts of lane width on crashes. 

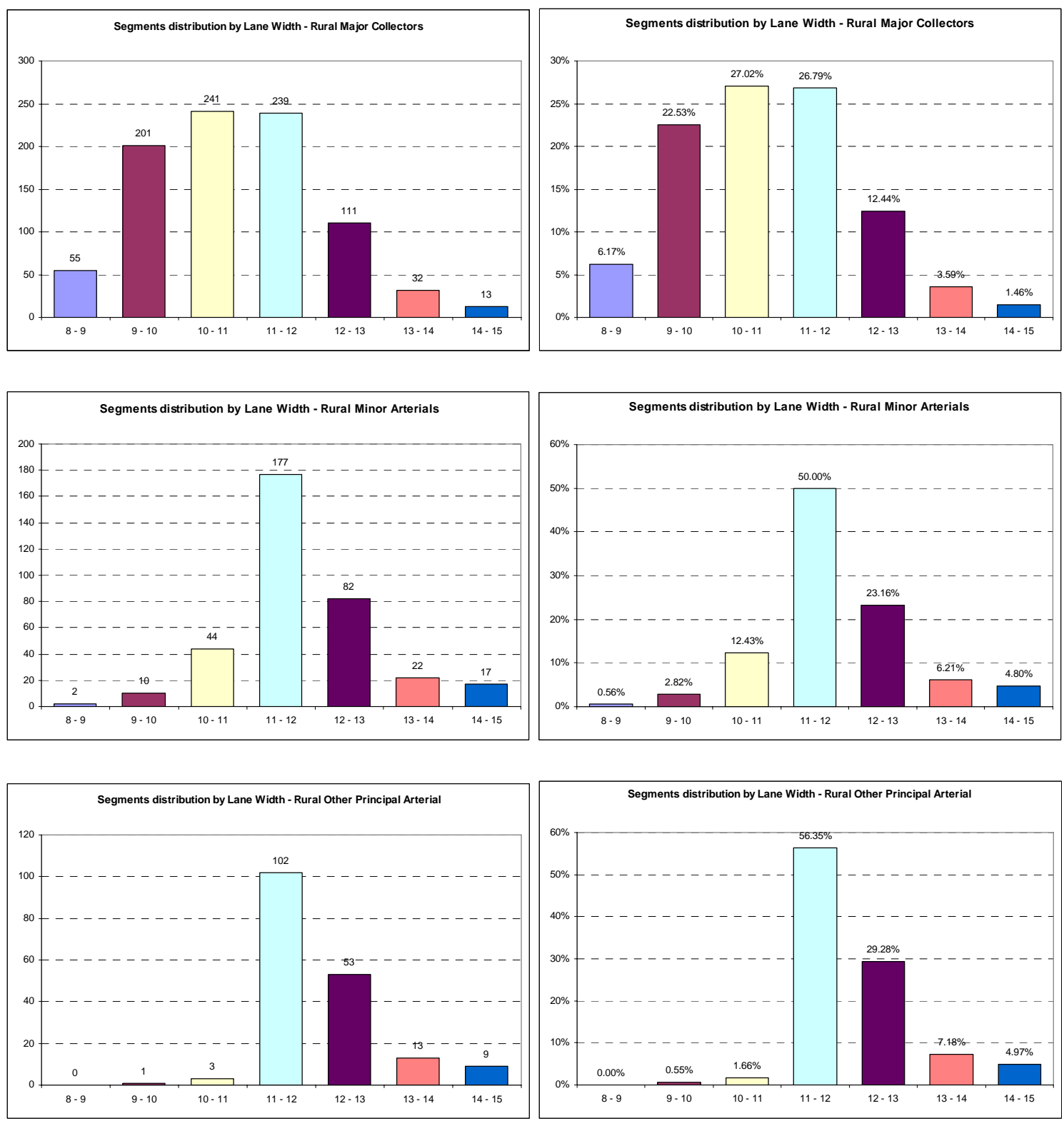

Figure 6-4: Distribution of Lane Widths at Rural Two-lane Highways in Indiana, by Functional Class 

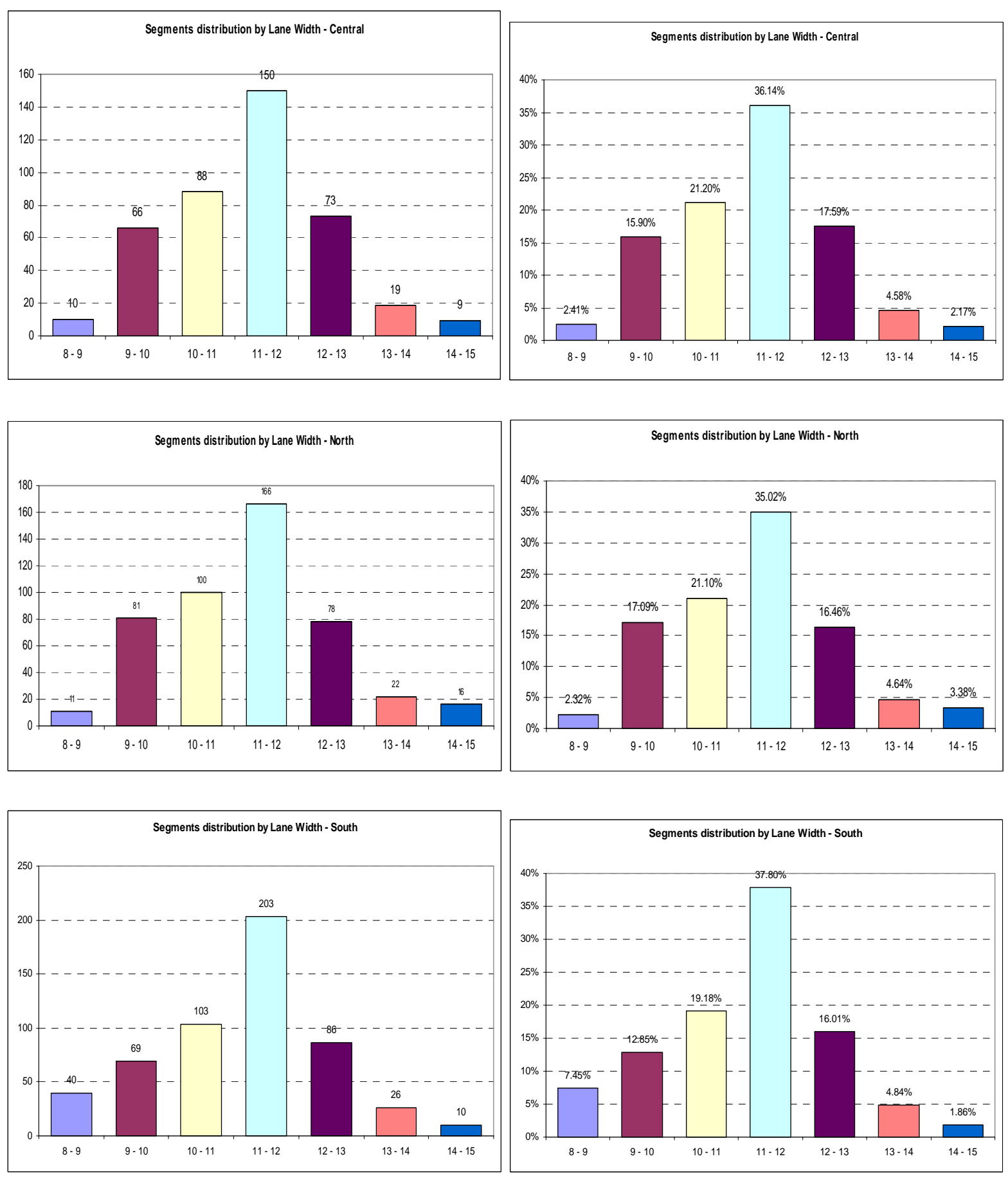

Figure 6-5: Distribution of Lane Widths at Rural Two-lane Highways in Indiana, by Region 


\subsubsection{Variation of Crash Rates with Lane Width for Indiana's Rural Two-lane Highway, by Functional Class}

A preliminary descriptive analysis of the variation of crash rates with shoulder widths was carried out using 1997-1999 data from Indiana. The results (Figure 6-6) clearly showed that increasing lane widths is generally associated with decreasing crashes. The ordinate axis shows the average annual crash rate per vehicle-miles-of-travel. The lane widths were grouped in bins of: very small, small, medium, and large. The observed trend was the same for all crashes, casualty crashes (fatal plus injury), and property-damage-only crashes. It is also seen that the results were generally consistent for all three functional classes of rural two-lane highways (principal and minor arterials, and major collectors).

It is also seen that irrespective of crash severity type, crash rates seem to decrease as functional class "increases" from collectors to principal arterials. From major collectors to minor arterials, there is generally a small decrease in crash rates, and from minor arterials to principal arterials, there is generally a large drop in crash rates.

It is also seen that lower classes of roads appear to be more sensitive to increases in lane widths, as increasing lane widths (from small to large) is associated with relatively higher returns in terms of crashes saved.
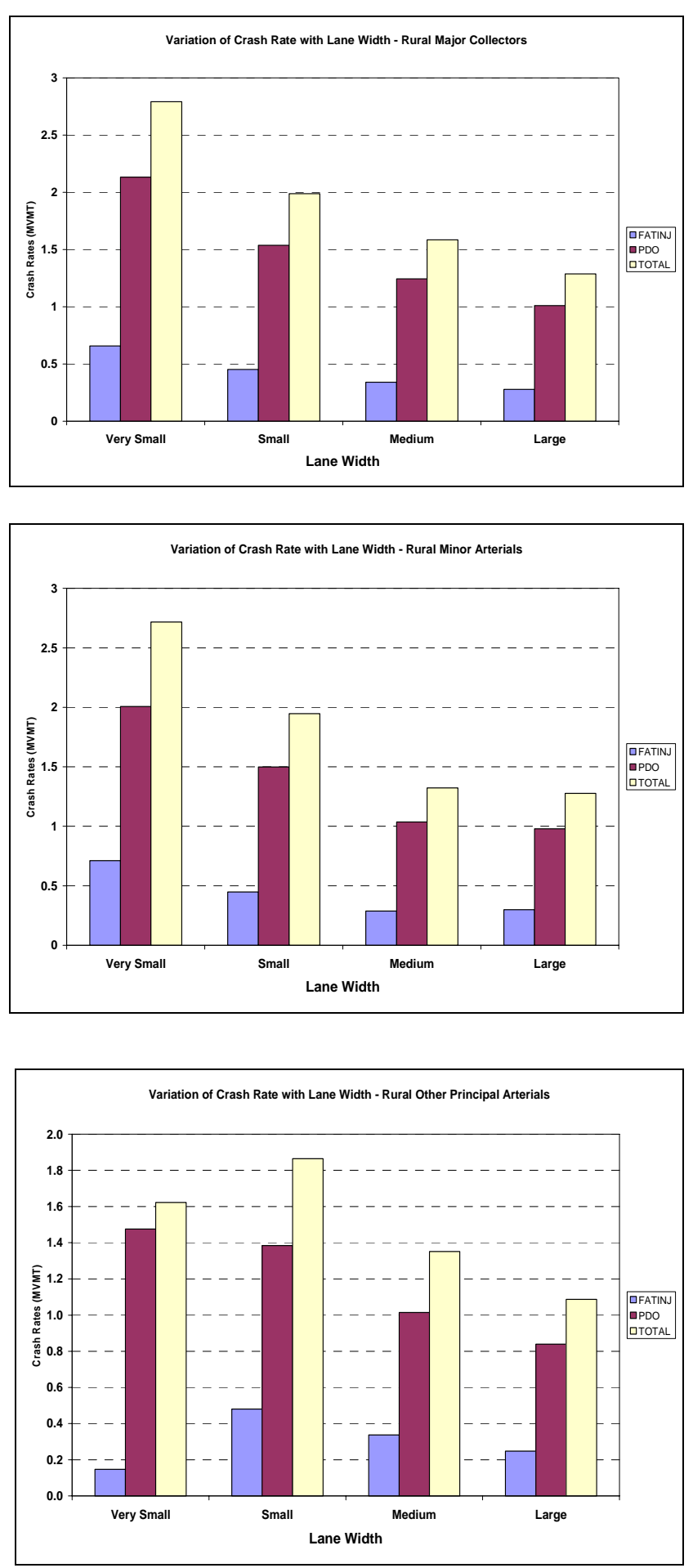

Figure 6-6: Variation of Crash Rates with Lane Width by Crash Severity and Functional Class 
From the Indiana data used in the present study, the correlation coefficients between lane width and crash rates are presented in Table 6-2 below. The negative sign of the coefficients support the findings from the descriptive analysis: greater lane widths are associated with reduced crash rates (crashes per VMT). The correlation matrix also indicates that there is a stronger relationship between lane width and crash rates for injury and property-damage-only crashes, compared to such relationship for fatal crashes. This suggests that any lane-widening effort is likely to lead to a relatively large reduction in injury and PDO crashes, but relatively small reduction in fatal crashes. The hypothesis for such relationship trends was investigated and tested for statistical significance during the model development (Chapter 6).

Table: 6-2: Correlation Matrix for Lane Widths and Crashes

\begin{tabular}{|c|c|c|c|c|c|}
\hline & $\begin{array}{c}\text { Fatal Crash } \\
\text { Rate }\end{array}$ & $\begin{array}{c}\text { Injury } \\
\text { Crash Rate }\end{array}$ & $\begin{array}{c}\text { PDO } \\
\text { Crash Rate }\end{array}$ & $\begin{array}{c}\text { Fatal or } \\
\text { Injury } \\
\text { Crash Rate }\end{array}$ & $\begin{array}{c}\text { Total Crash } \\
\text { Rate }\end{array}$ \\
\hline $\begin{array}{c}\text { Lane Widths of } \\
\text { Rural Two-Lane } \\
\begin{array}{c}\text { State Highways in } \\
\text { Indiana }\end{array}\end{array}$ & -0.0619 & -0.3217 & -0.3226 & -0.3215 & -0.3480 \\
\hline
\end{tabular}

\subsubsection{Pavement Friction}

Friction number values for the rural two-lane state highway sections were supplied by the Research Division of INDOT. Such measurements are obtained from tests performed at periodic time intervals at randomly selected sections on the state highway system. This test, referred to as the skid resistance test, measures the coefficient of wet sliding (skidding) friction at $40 \mathrm{mph}$ between a wet pavement surface and a standard tire. It is expected that increasing pavement friction would be associated with decreased crashes because pavement friction helps stop a vehicle that is approaching a hazardous situation on the roadway.

\subsubsection{Distribution of Pavement Friction at Rural Two-lane Highways in Indiana, by Functional Class and Region}

Using data supplied by INDOT Research Division, the distribution of average pavement friction by functional class and region were investigated as part of the present study. The following ranges were used for the categorization of the section pavement friction: 


$\begin{array}{llr}\text { Poor } & = & 0<F R \leq 4 \\ \text { Fair } & = & 4<F R \leq 8 \\ \text { Good } & = & 8<F R \leq 12 \\ \text { Very Good } & = & F R>12\end{array}$

The results (shown as Figure 6-7) are indicative of rather glaring deficiencies on lower class roads: almost $50 \%$ of all sections on rural major collectors have very low friction values. On the other hand, only $25 \%$ of rural principal arterials have low friction values. The distribution of friction values seems to approach a normal distribution (with a central tendency at the "low" friction value) as one moves from the lower class roads (major collectors) to the higher class roads (principal arterials).

Between Northern and Central Indiana, there seem to be similar distribution in average pavement friction, with the North having a slight edge over the Central (Figure 6-8). Both exhibit a left-skewed distribution with most observations falling in the "low friction" category. The case for the South seems rather poor: over $50 \%$ of Southern pavements have a "low" friction value, and only approximately $10 \%$ can be described as having medium or high friction.

The correlation matrix (Table 6-3) suggests that increasing surface friction is associated with deceasing crashes, which is intuitive.

Table 6-3: Correlation Matrix for Pavement Surface Friction and Crash Occurrence

\begin{tabular}{|c|c|c|c|c|c|}
\hline & $\begin{array}{c}\text { Fatal Crash } \\
\text { Rate }\end{array}$ & $\begin{array}{c}\text { Injury Crash } \\
\text { Rate }\end{array}$ & $\begin{array}{c}\text { PDO } \\
\text { Crash Rate }\end{array}$ & $\begin{array}{c}\text { Fatal or Injury } \\
\text { Crash Rate }\end{array}$ & $\begin{array}{c}\text { Total Crash } \\
\text { Rate }\end{array}$ \\
\hline $\begin{array}{c}\text { Pavement Surface } \\
\begin{array}{c}\text { Friction of Rural } \\
\text { Two-Lane Highways } \\
\text { in Indiana }\end{array}\end{array}$ & -0.0381 & -0.0981 & -0.1175 & -0.0928 & -0.1201 \\
\hline
\end{tabular}



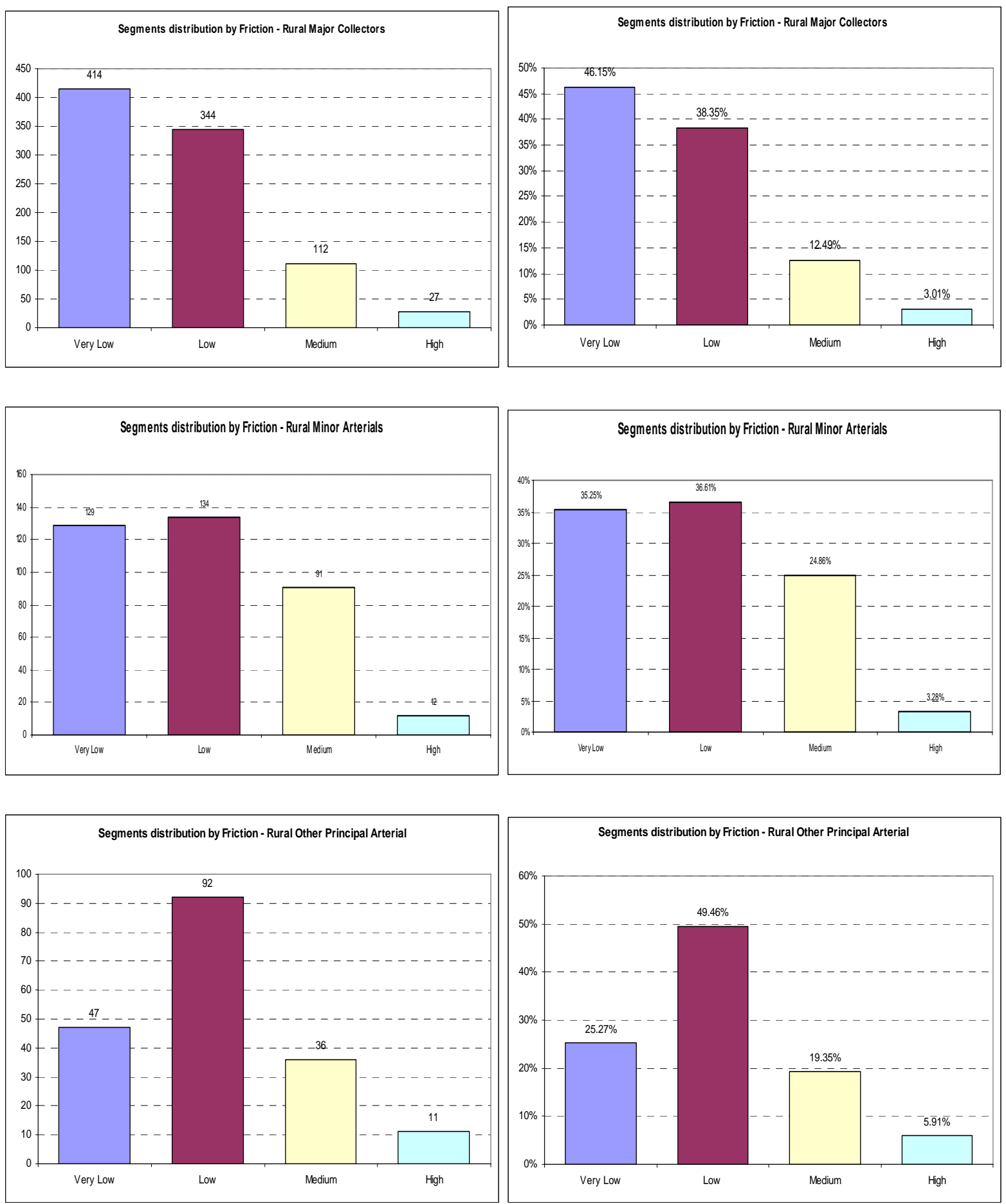

Figure 6-7: Distribution of Average Pavement Friction at Rural Two-lane Highways in Indiana, by Functional Class 

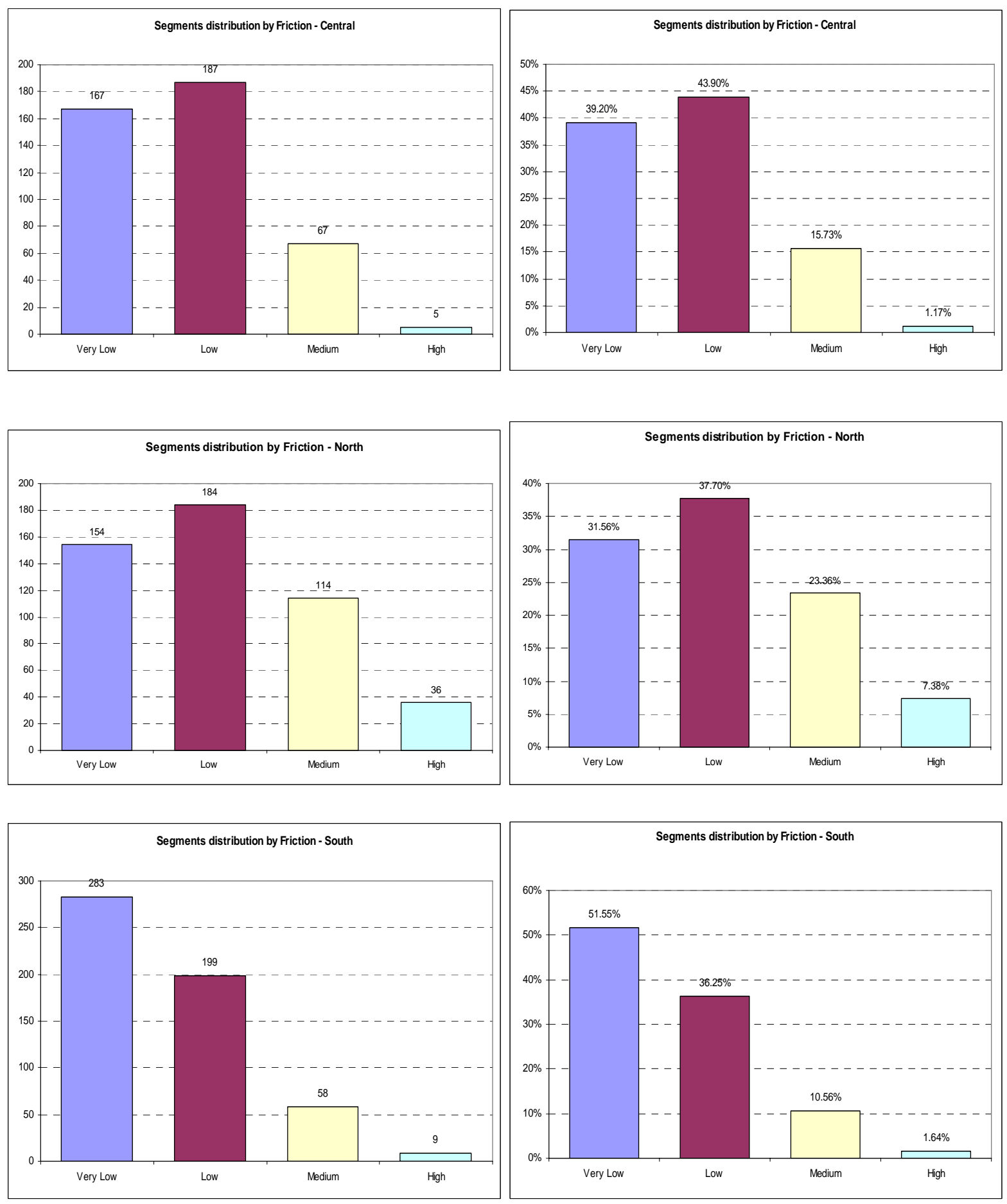

Figure 6-8: Distribution of Average Pavement Friction at Rural Two-lane Highways in Indiana, by Region 


\subsubsection{Variation of Crash Rates with Pavement Friction by Functional Class}

\section{Using Year 2000 pavement friction} data made available by INDOT Research Division, a preliminary descriptive analysis of the variation of crash rates with surface friction was carried out (Figure 6-9). The ordinate axis shows the average annual crash rate per VMT. The surface friction values were grouped in various bins as shown.

For casualty (fatal+injury) crashes on rural major collectors, the results were intuitive and unequivocal: increasing pavement friction is associated with decreasing crashes. For PDO crashes, a similar trend was observed with the exception of a slight increase in PDO crashes for very high values of pavement friction. The trends for rural minor arterials were somewhat inconclusive, even though a slight general decrease in crashes with increasing pavement friction may be discerned. For rural principal arterials, there seem to be a decrease in crashes with increasing friction only from the fair friction to the very good friction range, and only for total and PDO crashes.

It is also seen, irrespective of crash severity type, that crash rates seem to decrease as functional class "increases" from collectors to principal arterials. It is also apparent that lower classes of roads are more sensitive (in terms of crash rate reduction) to increasing levels of pavement friction.
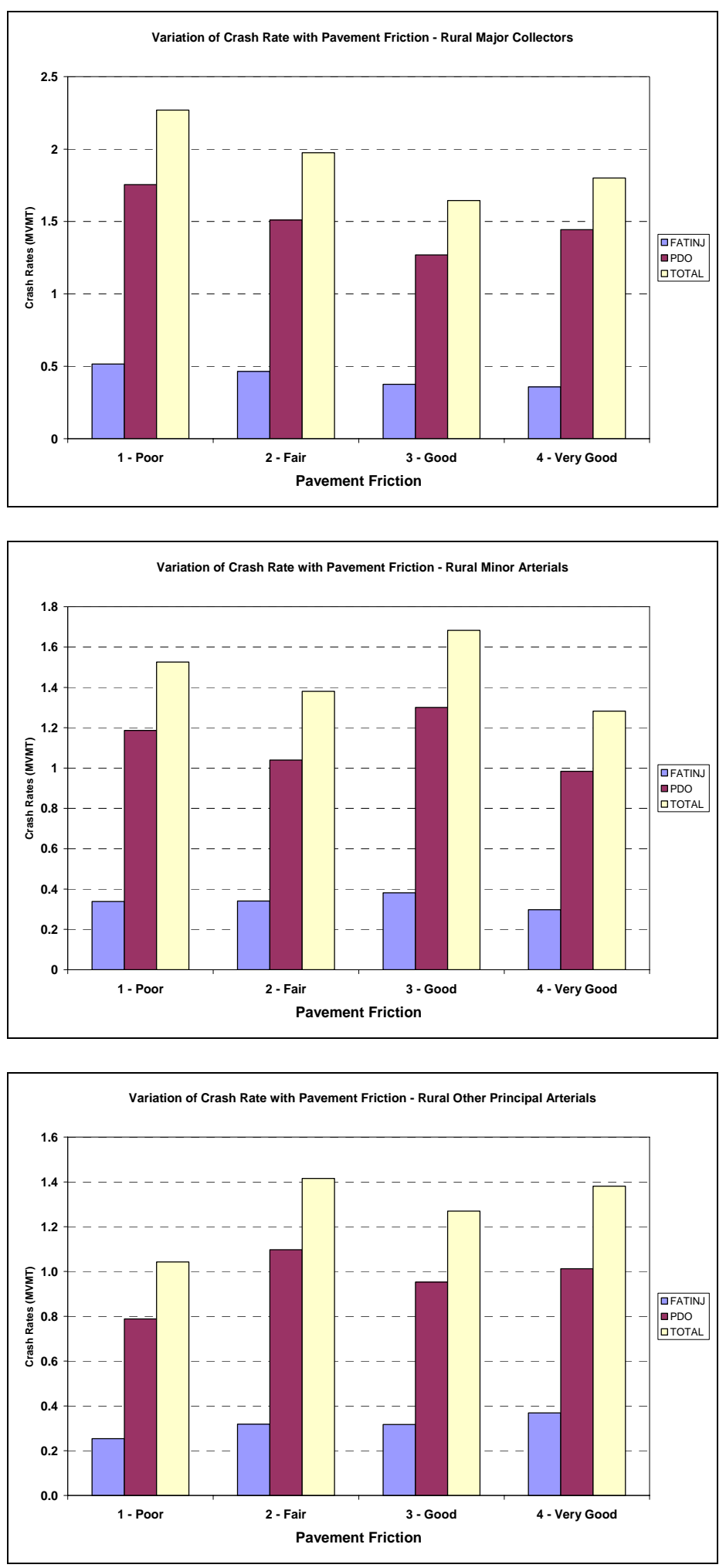

Figure 6-9: Variation of Crash Rates with Pavement Friction by Crash Severity and Functional Class 


\subsubsection{Pavement Condition}

Present Serviceability Index (PSI), a measure of pavement surface condition, is measured periodically at state highway sections by INDOT's Program Development Management Division. Roughness numbers computed from the field data are correlated to ride ratings, which are commonly referred to as PSI on a scale of $0-5$ (5 is the rating value for a very good pavement surface). Generally, crash rates tends to decrease as pavement condition improves from poor (PSI value $=2$ ) to good (PSI value =4), and then increases beyond this value. This may be attributed to the fact that very good pavement conditions are generally associated with higher speeds and subsequently higher crash rates. But does pavement resurfacing really increase safety? This has been the subject of much debate. Some researchers contend that resurfacing a road increases speeds, and consequently increases crashes. Others maintain that such effects are yet to be proven without reasonable doubt [Harwood et al., 2003]. Hauer et al. [2000] demonstrated that there is a positive impact of resurfacing on safety but may exist for only 30 months for non-intersection crashes. NCHRP Project 17-9(2) reported that resurfacing had a negative effect on safety at certain states and a positive effect at others.

It seems plausible that resurfacing results in both impacts: a positive impact due to improved friction and roughness, and a negative impact due to tendency of drivers to increase speeds on new smooth roads. As such, the net impact for a given pavement may be positive or negative depending on factors such as the initial pavement condition, jump in condition, or type of crash. The correlation matrix established using data for rural two-lane roads in Indiana exhibited a similar inconclusive trend (Table 6-4): for some crash types, increased pavement condition is associated with increased crashes, while the converse was true for other crash types.

\subsubsection{Distribution of Pavement Condition, Rural Two-lane Highways in Indiana, by Functional Class and Region}

For the rural two-lane state highways in Indiana, PSI data obtained from the roadway inventory file shows that pavement condition PSI is generally highest at rural principal arterials and lowest for the rural collectors. Examination of regional variations shows that Northern and Central pavements appear to be in better condition than Southern pavements. The following ranges were used for categorizing the pavement condition of the sections:

$\begin{array}{llr}\text { Very Poor } & = & 0<P S I \leq 1 \\ \text { Poor } & = & 1<P S I \leq 2 \\ \text { Fair } & = & 2<P S I \leq 3 \\ \text { Good } & = & 3<P S I \leq 4 \\ \text { Very Good } & = & P S I>4\end{array}$


Table 6-4: Correlation Matrix for Surface Condition and Crash Rates

\begin{tabular}{|c|c|c|c|c|c|}
\hline & $\begin{array}{c}\text { Fatal Crash } \\
\text { Rate }\end{array}$ & $\begin{array}{c}\text { Injury Crash } \\
\text { Rate }\end{array}$ & $\begin{array}{c}\text { PDO } \\
\text { Crash Rate }\end{array}$ & $\begin{array}{c}\text { Fatal or Injury } \\
\text { Crash Rate }\end{array}$ & $\begin{array}{c}\text { Total Crash } \\
\text { Rate }\end{array}$ \\
\hline $\begin{array}{c}\text { Pavement Surface } \\
\text { Condition of Rural } \\
\begin{array}{c}\text { Two-Lane Highways } \\
\text { in Indiana }\end{array}\end{array}$ & -0.0032 & -0.0215 & 0.0080 & -0.0215 & 0.0005 \\
\hline
\end{tabular}

\subsubsection{Inadequacy of Passing Opportunities}

Rural two-lane state highways and county roads in Indiana that carry relatively high traffic volumes obviously experience significant levels of operational difficulties and safety problems that are exacerbated by inadequate geometry (steep grades and poor sight distance, particularly in southern Indiana), and lack of passing opportunities due to oncoming traffic and/or poor sight distance. Passing inadequacy becomes manifest at a two-lane road section when the demand for passing opportunities exceeds their supply [Fitzpatrick et al., 2000]. The supply of passing opportunities depends on the availability of passing sight distance and gaps in the opposing traffic stream. When demand exceeds supply, fast-moving vehicles catch up with slower ones, and are unable to pass. The provision of passing facilities can increase the opportunity for the faster vehicles to pass the slower ones, and lack of such facilities has been associated with higher crash frequencies [Fitzpatrick et al., 2000]. To address operations and safety problems posed by inadequate passing facilities, five strategies identified in the literature are as follows:

- passing lanes

- climbing lanes

- short four-lane sections

- turnouts

- shoulder use sections

These countermeasures, illustrated as Figure 6-10, are discussed below:

\subsubsection{Passing Lane, Climbing Lane, and Short Four-lane Section:}

A passing lane is an added lane provided in one or both directions of travel on a conventional twolane highway to improve passing opportunities, and includes passing lanes on level or rolling terrain, climbing lanes on grades, and short four-lane sections. The length of an added lane may vary from 1,000 ft to 3 miles [Fitzpatrick et al., 2000]. 


\subsubsection{Turn-out.}

A turn-out is a widened, unobstructed shoulder area on a two-lane highway that allows slow-moving vehicles to pull out of the through lane to permit following vehicles to pass. Typically, turnouts are relatively short (less than $600 \mathrm{ft}$ in length), and are typically more relevant at winding road sections in mountainous terrain such as those found in southern Indiana.

\subsubsection{Shoulder Use Section:}

Driving on road shoulders is generally illegal in most states. However, paved shoulders could be used for enhancing passing operations by placing signs at locations where such practice may be permitted [Fitzpatrick et al., 2000]. Also, shoulder use in such manner could be restricted to daylight hours only.

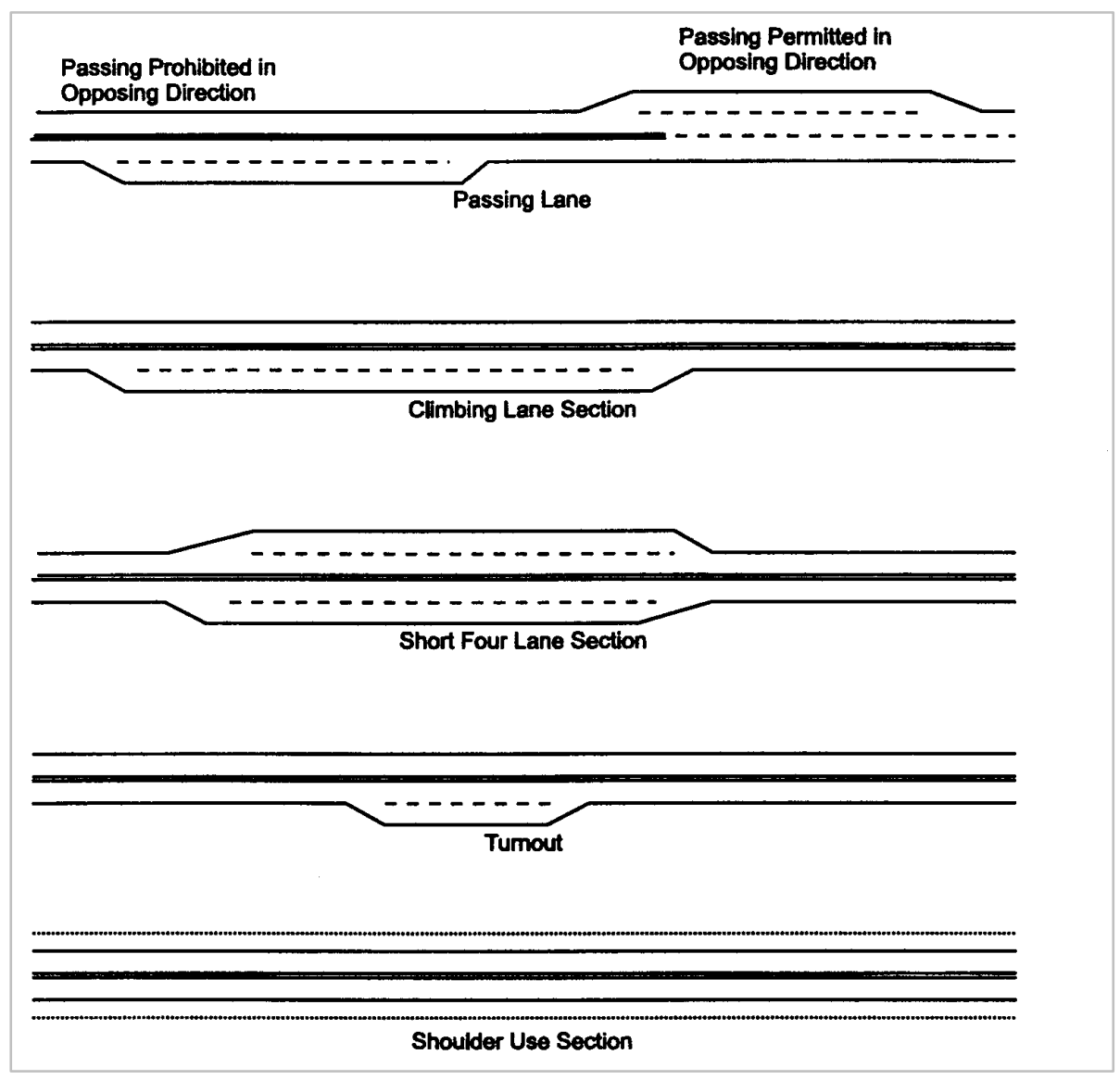

Figure 6-10: Typical Treatments for Addressing Passing Operations on Rural 2-Lane Roads

[Fitzpatrick et al., 2000] 
Table 6-5 presents the effectiveness of the above countermeasures in addressing safety problems associated with inadequate passing opportunities. Harwood et al. [1987] reported reductions in crash frequencies of $25-40 \%$ were reported for passing lanes, short four-lane sections, and turn-out lanes, but cautioned that these values were for two-lane roads with traffic volumes higher than the volume typical of such roads. The researchers cautioned that while such alternatives address safety problems, other problems may be created by their use, and they consequently provided detailed guidelines on the implementation of such alternatives.

Table 6-5: Effectiveness of Alternative Designs for Passing Opportunities

\begin{tabular}{|l|c|c|c|}
\hline \multirow{2}{*}{$\begin{array}{c}\text { Amount of Lane Widening } \\
(\mathrm{ft})\end{array}$} & \multirow{2}{*}{ Type of Area } & \multicolumn{2}{|c|}{$\%$ Reduction in Crashes } \\
\cline { 3 - 4 } & & Total Crashes & Casualty Crashes (Fatal + Injury) \\
\hline Passing Lanes & Rural & 16 & 13 \\
\hline Short Four-lane Section & Rural & 29 & 25 \\
\hline Turnout & Rural & 40 & 35 \\
\hline Shoulder Use Section & Rural & 49 & 43 \\
\hline
\end{tabular}

Note: These values are only for rural two-lane roads.

Source: [Harvood et al., 1987]

A descriptive analysis of the distribution of sections with lack of passing opportunities and the variation of such defective designs with crash rates for Indiana's rural two-lane state highways, could not be carried out due to lack of data. The current database does not indicate where such problems exist on the network. As INDOT's safety management unit continues to collect safety data for all sections on the state highway network, it is expected that passing opportunity considerations can be considered in a future similar study.

\subsubsection{Narrowing of Roadway at Bridge Sites}

In many instances, roadways are widened to accommodate increased traffic but bridges at such sections may be left unwidened because of lack of funds, jurisdictional issues, or some other reason. Bridges in such condition are said to be functionally obsolete. Anecdotal evidence suggests that this situation is relatively common for INDOT's rural two-lane roads of lower class (major and minor collectors). The safety situation at such bridges may be further exacerbated where there is poor signing and delineation, and inadequate sight distance at the bridge approaches (such as when the bridge is located just past a sharp horizontal curve). The impact of poor signing has been studied in 
literature and is discussed in a separate section of this chapter. However, relatively very few studies have investigated the impact of bridge functional inadequacy on safety, and it was found that a key factor is the difference between the approach width and the bridge width (also referred to as the "relative" bridge width) as illustrated in Figure 6-11.

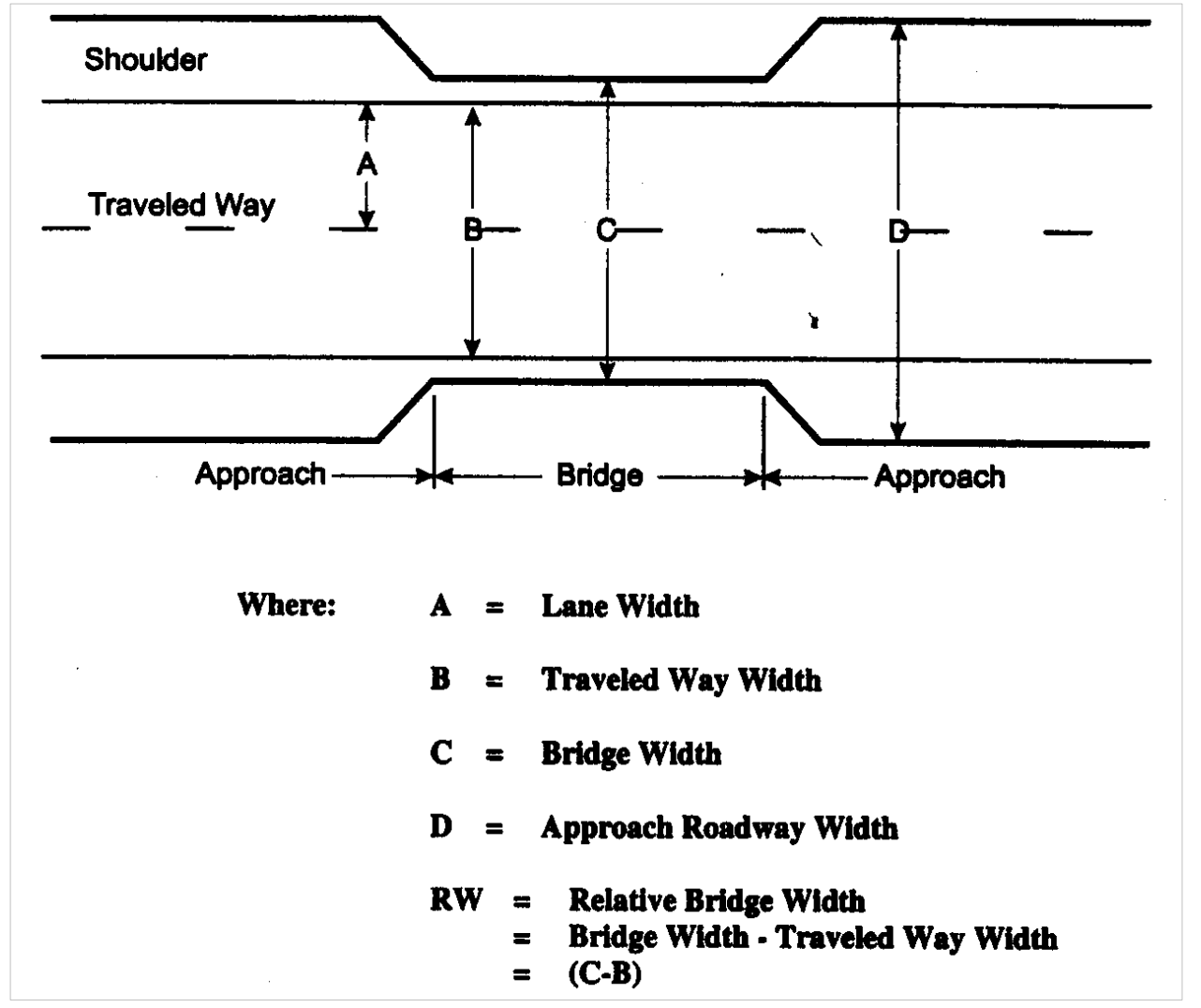

Figure 6-11: The Concept of "Relative" Bridge Width [Turner, 1984]

Using crash and inventory data at over 2,000 bridges at two-lane roads in Texas, Turner [1984] determined that a relationship exists between the relative bridge width and bridge crashes (Figure 6-12). Turner showed that the crash rate decreases as the relative bridge width increases. Also, it can be inferred from the figure that it is desirable to have bridge widths of at least $6 \mathrm{ft}$ wider than the approach width (in other words, shoulder width at the bridge should be at least $3 \mathrm{ft}$ ). In that study, the dataset used for the modeling did not include similar bridges with zero crashes, and the resulting model may have adversely been affected by the econometric phenomenon known as selectivity bias. Nevertheless, the Turner study is touted as the best information on the subject. 


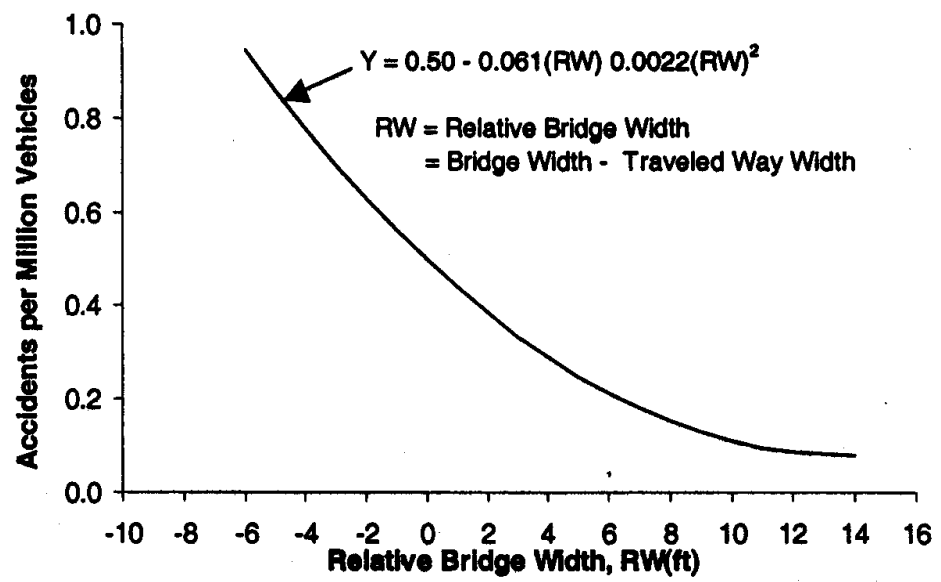

Figure 6-12: Relationship between Crash Rate and "Relative" Bridge Width [Turner, 1984]

Table 6-6: Summary of Accident Reduction Factors Associated with Widening Shoulders on Bridges

\begin{tabular}{|c|c|c|c|c|c|c|}
\hline \multirow{2}{*}{$\begin{array}{c}\text { Bridge Shoulder Width on each side } \\
\text { before Widening (ft) }\end{array}$} & \multicolumn{6}{|c|}{ Bridge Shoulder Width after Widening (ft) } \\
\cline { 2 - 7 } & 2 & 3 & 4 & 6 & 7 & 8 \\
\hline 0 & 23 & 42 & 57 & 78 & 83 & 85 \\
\hline 1 & - & 25 & 45 & 72 & 78 & 80 \\
\hline 2 & - & - & 27 & 62 & 71 & 74 \\
\hline 3 & - & - & - & 48 & 60 & 64 \\
\hline 4 & - & - & - & 44 & 44 & 50 \\
\hline
\end{tabular}

Note: Width of bridge lanes assumed constant.

$V$ alues derived from crash model developed for rural two-lane roads by Turner, 1984]

Source of table: Fitzpatrick et al. [2000]

Countermeasures typically used to address the problem of such functional obsolescence of bridges include:

- Installation/repair of bridge railings

- Increase bridge width

- Improve signing and delineation 


\subsubsection{Installation/ repair of bridge railings}

Bridge and culvert structures are required to have some protective facility such as railing, concrete barrier or headwall in place to prevent vehicles from running off their edges. In its Roadway Design Guide, AASHTO [1996] states that bridge rails designed to pre-1964 AASHTO specifications may not meet current specifications and may need retrofitting. Such retrofitting may include any of the following activities: reinforcing the strength of the railing or protective system, providing longitudinal continuity, and increasing the height of the rail systems to accommodate higher profile vehicles such as SUVs (Fitzpatrick et al., 2000].

\subsubsection{Increase bridge width}

Increasing bridge width is probably the most effective, yet most expensive option for addressing typical problems of functional (width) obsolescence. The consistent and continuous alignment offered by a widened bridge contributes to improved safety (Plate 6-1). Table 6-6 presents the expected crash reduction factors (percent reductions) due to bridge widening.

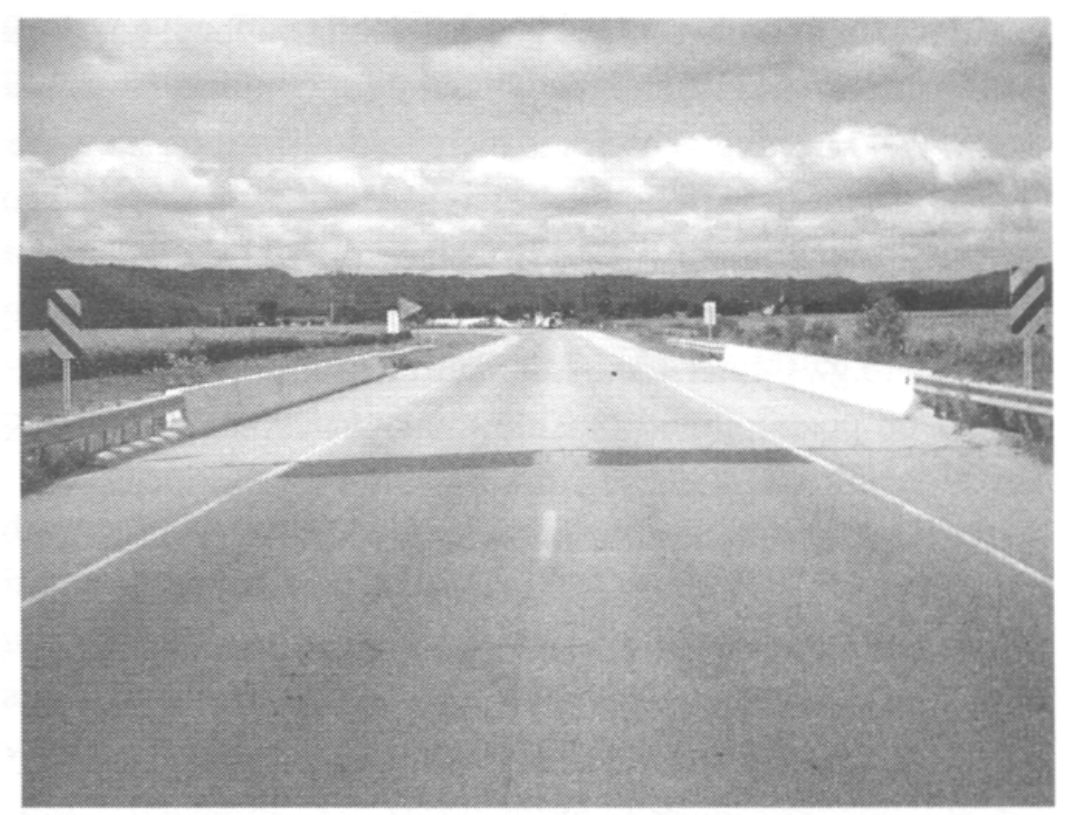

Plate: 6-1: Bridge with Zero "Relative Width" [Fitzpatrick et al., 2000]

\subsubsection{Improve signing and delineation}

This is probably the least expensive of the options for addressing the problem of narrow bridges. Ullman and Pezdolt [1997] carried out a Texas study to investigate the impact various types and levels 
of bridge approach delineation (type and amount) on the comfort levels of drivers approaching and crossing selected narrow bridges. The study recommended the use of tapered edge-lines and transverse marking arrangements. For example, for a bridge with current lane and shoulder widths of

$10 \mathrm{ft}$ and $2 \mathrm{ft}$, respectively, it was estimated that widening the bridge shoulder to $6 \mathrm{ft}$ would yield a $62 \%$ reduction in crashes.

For the present study, a descriptive analysis of the distribution of sections with inadequate bridge width and the variation of such defective situations with crash rates could not be carried out due to lack of data. The current database does not indicate where such problems exist on the network. In a future study, bridge width data could be collected from Indiana's Bridge Management System database and matched with road width data from INDOT's roadway inventory file for comparison and identification of sections with bridge functional obsolescence. That way, data can be generated for a detailed analysis of this problem with regard to Indiana's rural two-lane roads.

\subsection{SECTIONAL AND ALIGNMENT FACTORS}

Sectional and alignment factors include section length, section traffic volume, horizontal alignment, and vertical alignment.

\subsubsection{Section Length}

Measured to the nearest hundredth (0.01) of a mile, section length for each road section refers to the distance between major intersections or where there is a major change in the engineering characteristics of the roadway. For purposes of crash modeling for the rural two-lane state highway segments in Indiana, all crashes within a specified distance from the intersections were excluded from the analysis because such crashes were assumed to be associated with intersections rather than the road segments.

As section length increases, all other factors remaining the same, the number of crashes is expected to increase. However, such relationship is not necessarily linear. For example, if sections are defined by the stretch between intersections, then doubling the section length may likely result in a crash frequency that is less than twice the original frequency. That is, as section length increases, the rate of crashes generally decreases, all other factors being the same, due to relatively large fraction of the zone of traffic flow conflicts and entropy associated for shorter road sections. The dataset used for study spanned 1997 to 2000 . However, for section lengths, data from the 1998 roadway inventory file was used for all years (1997-2000) because physical roadway characteristics (particularly, section lengths) experience little or no changes over time. In the Indiana dataset used for the present study, 
section lengths ranged from 0.19 miles to 9.97 miles with a mean and standard deviation of 5.09 and 2.20 , respectively.

A section of rural two-lane road may stretch from one county line to another and may need breaking up for purposes of analysis. What criteria should be used for breaking up such sections into smaller segments to serve as the main experimental unit for the analysis? This was an interesting issue that was given exhaustive consideration in the current study. The longer a segment used for the analysis, the more likely that it will have variations in geometric characteristics that will serve to obscure relationships between crash trends and geometric characteristics. On the other hand, from the sheer law of numbers, it is always better and easier to obtain patterns from a longer stretch than a shorter one, all else being equal. In other words, it is most desired to have long segments that are as uniform as possible for the purpose of the analysis, but it is difficult to obtain rural two-lane sections that exceed 10 miles in length and yet have uniform geometric features. Decreasing the lengths of the sections has the attractive merit of ensuring (to some extent) uniform geometric features, but the smaller a segment gets, the more sparse the crash data and consequently, the less reliable the analysis. Furthermore, the smaller a segment becomes, the more difficult it is to assign a crash (in the current crash database) to that segment. It is for these reasons that the research team chose not to utilize the shorter segments available in the roadway inventory file, but rather proceeded to utilize somewhat larger sections divided on the basis of their rural/urban limits.

\subsubsection{Section Traffic Volume}

The traffic volume for each road section is the average annual daily traffic (AADT) on the section over the four year period 1997 to 2000 . Where the section consists of segments with different AADTs, the weighted average of the AADT over the entire section was estimated and used for the analysis.

Section AADT is a very influential variable on crash frequencies. All other factors being equal, a higher AADT results in a higher number of crashes, at least up to the point where capacity is reached. However, inclusion of AADT in crash modeling must be done with a great deal of circumspection because it is typically correlated with a number of explanatory variables such as lane width, shoulder width, shoulder type and pavement friction. In other words, higher volume roads are likely to have superior geometric characteristics, even within a given functional class. Such correlations were evident in the preliminary investigations for the present study. AADT for the rural two-lane road segments in Indiana range from 161 to 23,340 vehicles per day, with a mean and 
median of 3,754 and 3,084, respectively. The high variability associated in the traffic volumes was further evidenced by the rather high standard deviation of 2,961 vehicles per day. The plots shown in the Appendix, suggest that increasing traffic volume is associated with increasing crashes but beyond a certain point, increased traffic volume is actually associated with decreased crashes. While such trends may be best explained using detailed section-by-section analysis, the overall trends may be attributable partly to the crash-reducing effects of lower speeds associated with increased congestion.

\subsubsection{Horizontal Alignment}

It has been shown in past research that horizontal curves experience crash rates of up to 4 times the rates on tangent sections, all else being equal [FHWA, 1988, AASHTO, 1994, Zegeer, 1987]. Zegeer [1987] identified the following traffic, roadway, and geometric features that influence safety at horizontal curve sections:

- Traffic volume on the curve and traffic mix (such as the percentage of trucks)

- Curve features (such as degree of curve, curve length, superelevation, presence of transition curves)

- Cross sectional curve element (such as lane-width, shoulder width, shoulder type, shoulder slope)

- Curve section roadside hazard features (such as clear slope, rigidity, and types of obstacles)

- Stopping sight distance on curve (or at curve approach)

- Vertical alignment on horizontal curve

- Distance to adjacent curves

- Distance of curve to nearest intersection, driveway, etc.

- Pavement friction

- Presence and type of traffic control devices (signs and delineation).

It has been shown by a number of researchers [Glennon et al., 1985; Zegeer et al., 1991; Glennon, 1987] that milder curves are associated with lower crash rates compared to sharper curves. For horizontal curves, casualty crashes seem to be more dominant than PDO crashes. Those researchers also found that horizontal curves seem to have proportionately more head-on and opposite direction sideswipe crashes, fixed object, crashes, rollover crashes and nighttime crashes compared to other sections. Glennon et al. [1985] and Fink and Krammes [1995] determined that the degree of curvature is the best predictor of crashes at curved sections. 
Geometric improvements used to improve safety at deficient horizontal curves include the following:

- Curve straightening

- Roadway widening at curve sections

- Superelevation improvements

- Roadside improvements at curve sections

Also, traffic control devices are typically used to provide warning to curve approaches and to provide delineation of the pavement. These are discussed in a different section of this chapter.

In their 1983 study, Glennon et al. developed a discriminant model for use in identifying potentially hazardous horizontal curve sites on the basis of geometric, traffic, and roadside features and conditions. The study found that hazardous roadside designs are the primary cause of crashes at horizontal curve sections. Fink and Krammes investigated the effect of degree of curvature, tangent length, and sight distance on accident rates at horizontal curves, and found that crash occurrence at curve sections was significantly influenced by the degree of curvature (Figure 6-13). According to the researchers, variables not found statistically significant include preceding tangent length, sight distance, lane width, pavement width and state.

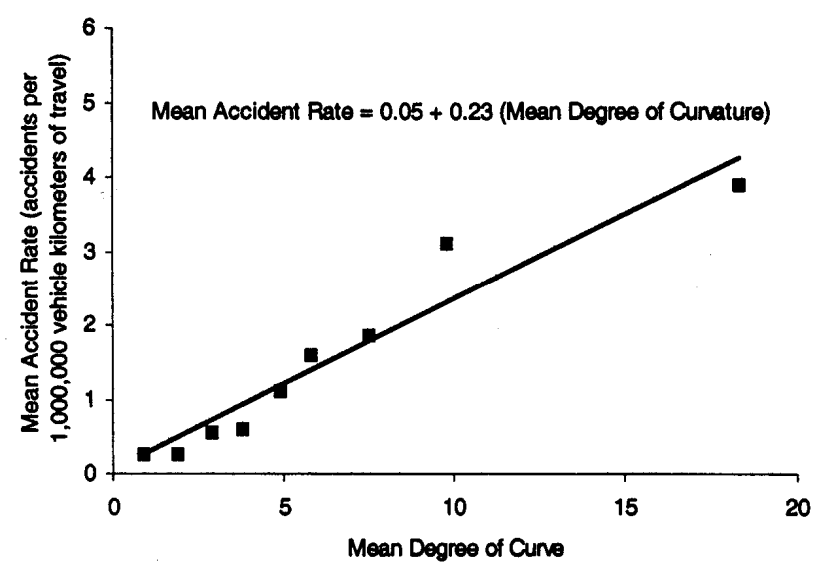

Figure 6-13: Relationship between Mean Crash Rate and Mean Degree of Curvature

In a 1991 study by Zegeer et al. for the FHWA, the impacts of various geometric elements on crashes were investigated using a database comprising over 10,000 curve sections, and the findings were generally consistent with those of past studies as discussed earlier in this section. 
6.2.3.1 Curve Straightening: This refers to the reconstruction of a curve to make it "less sharp" by giving it a longer radius or a lower degree of curve. Based on the research carried out by Zegeer et al. [1991], the Interactive Highway Safety Design Model (IHSDM) accident prediction model for twolane highways uses the following accident modification factor (different from crash reduction factor) to determine the expected accident frequency of a horizontal curve relative to a tangent roadway:

$$
A M F=\frac{1.55 L_{C}+\frac{80.2}{R}-0.0125}{1.55 L_{C}}
$$

Where $L_{c}=$ length of the horizontal curve (miles), $R=$ radius of curvature (ft), $S=1$ if spiral transition is present, 0 otherwise.

For a straightened curve (increased radius) whose central angle remains the same, the length of the curve increases with such improvement. Fitzpatrick et al. [2000] caution that this consideration should be taken into account when evaluating the expected impacts of curve straightening on crashes: the expected crashes on the longer curve with larger radius should be compared with crashes on the shorter and sharper curve plus the two tangent sections on either end.

6.2.3.2 Widening at curve sections: Widened lanes and shoulders at curve sections are known to result in reduced crashes at curves. Zegeer et al [1991] provided values to estimate the expected effectiveness of curve widening activities, as shown in Table 6-7.

Table 6-7: Effectiveness of Curve Widening Activities

\begin{tabular}{|c|c|c|c|c|}
\hline $\begin{array}{c}\text { Total Amount of Lane or Shoulder } \\
\text { Widening at Curve (ft) }\end{array}$ & \multicolumn{3}{|c|}{ \% Reduction in Crashes } \\
\hline Total & Per Side & Lane Widening & $\begin{array}{c}\text { Paved Shoulder } \\
\text { Widening }\end{array}$ & $\begin{array}{c}\text { Unpaved Shoulder } \\
\text { Widening }\end{array}$ \\
\hline 2 & 1 & 5 & 4 & 3 \\
\hline 4 & 2 & 12 & 8 & 7 \\
\hline 6 & 3 & 17 & 12 & 13 \\
\hline 8 & 4 & 21 & 19 & 16 \\
\hline 10 & 325 & - & 21 & 21 \\
\hline 12 & 6 & - & 25 & 24 \\
\hline 14 & 7 & - & 28 & 26 \\
\hline 16 & 8 & - & 31 & 29 \\
\hline 18 & 10 & - & & 13 \\
\hline 20 & 9 & - & & \\
\hline
\end{tabular}

Note: These values are only for two-lane roads.

Source: [Zegeer et al., 1991] 
6.2.3.3 Superelevation Improvements: Superelevation refers to the banking of the roadway cross section in order to counter the effect of centrifugal forces that tend to push the vehicle of the roadway as it negotiates the curve. There have been a number of studies that have investigated the link between lack of superelevation (Plate 6-2) and crash frequency. Zador et al. [1985] found that there were deficiencies in superelevation at fatal accident sites compared to control sites. A 1991 FHWA study observed a discernible impact of inadequate superelevation [Zegeer et al. 1991]. The researchers determined that curve sites with superelevation deficiency (defined as the difference between the existing superelevation and that recommended by the AASHTO Green Book) exhibited significantly poorer crash experience compared to other otherwise similar sites, and presented expected reductions in curve crashes due to superelevation improvements (Table 6-8). It is also seen that generally improving superelevation at a curve section from 0 to 0.2 can yield a $6 \%$ reduction in crashes at that section.

Table 6-8: Effectiveness of Providing Superelevation at Horizontal Curves

\begin{tabular}{|c|c|}
\hline Superelevation Deficiency & Accident Modification Factor \\
\hline 0.00 & 1.00 \\
\hline 0.01 & 1.00 \\
\hline 0.02 & 1.06 \\
\hline 0.03 & 1.09 \\
\hline 0.04 & 1.12 \\
\hline
\end{tabular}

Note: These values are only for two-lane roads. Source: [Zegeer et al., 1991]

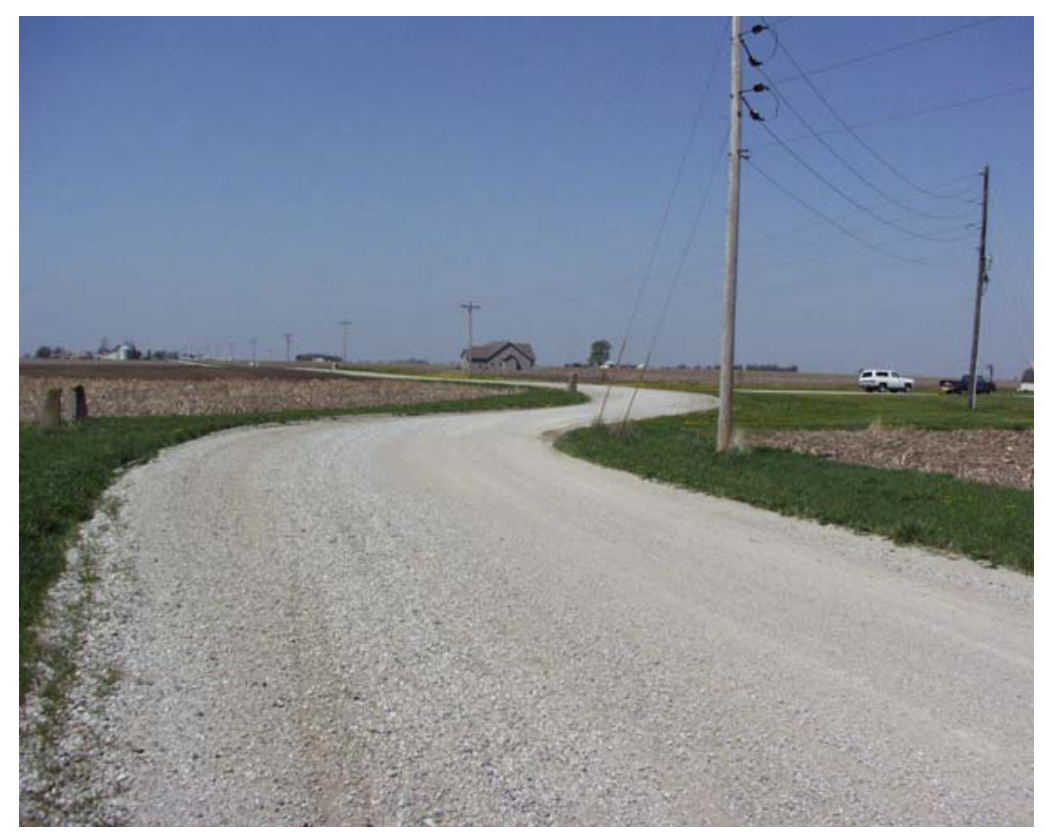

Plate 6-2: Superelevation and Pavement Material Stabilization May Help to Stabilize Vehicles

Negotiating Horizontal Curves at County Road Sections 
There is a practical limit to the rate of superelevation. In other words, excessive superelevation can be detrimental. AASHTO [1994] states that in areas subject to ice and snow, the rate of superelevation cannot be greater than that on which vehicles standing or traveling slowly would slide down the cross slope when the pavement is icy. When a vehicle travels slowly around a curve with high superelevation, negative lateral forces develop and the vehicle is held in the proper path only when the driver steers up the slope or against the direction of the horizontal curve. This direction of steering is an unnatural movement on the part of the driver and possibly explains the difficulty of driving on roads where the superelevation is in excess of that required for normal speeds. Also, some vehicles, such as large trucks, may have a high center of gravity or may be loosely suspended on their axles. When these vehicles travel slowly on steep cross slopes, a high percentage of the mass is carried by the inner tires. According to AASHTO, "where snow and ice are factors, tests and experience show that a superelevation rate of about 8 percent is a logical maximum to minimize slipping across a highway when stopped or attempting to slowly gain momentum from a stopped position." Zegeer et al. [1991] investigated the adverse impacts of excessive superelevation on crash rates, but found no such significant impacts with the available data.

A descriptive analysis of the distribution of curve sections with inadequate superelevation, and the variation of such defective situations with crash rates could not be carried out due to lack of data. The current databases do not indicate the magnitude of superelevation at horizontal curve sections. There are indications that efforts are currently in progress at INDOT to collect such data. As such, these issues could be investigated in a future study.

6.2.3.4 Roadside Improvements at Curve Sections: Zegeer et al, in their 1991 FHWA study, also investigated the relationships between crashes and roadside improvements at curve sections. The results of the study, which generated the crash reduction factors shown in Table 6-9, correspond to increasing the clear roadside recovery distance on a horizontal curve. The roadside improvements include tree removal, utility pole relocation, and flattening roadside slopes. The study results showed that an increase in recovery distance of $5 \mathrm{ft}$ would be expected to reduce total curve crashes by $9 \%$. Also, clearing the roadside of all obstacles within $20 \mathrm{ft}$ is expected to yield a total curve crash reduction of almost $30 \%$. 
Table 6-9: Effectiveness of Increasing Roadside Recovery Distance at Curve Sections

\begin{tabular}{|c|c|}
\hline $\begin{array}{c}\text { Increase in Roadside Clear } \\
\text { Recovery Distance (ft) }\end{array}$ & $\begin{array}{c}\text { Percent Reduction in } \\
\text { Total Curve Crashes }\end{array}$ \\
\hline 5 & 9 \\
\hline 8 & 14 \\
\hline 10 & 17 \\
\hline 12 & 19 \\
\hline 15 & 23 \\
\hline 20 & 29 \\
\hline
\end{tabular}

Source: [Zegeer et al., 1991$]$

Table 6-10: Accident Reduction Factors for Flattening Side-slopes on Curves

\begin{tabular}{|c|c|c|c|c|}
\hline \multirow{2}{*}{$\begin{array}{c}\text { Initial Side-slope of } \\
\text { Curve (before } \\
\text { Treatment) }\end{array}$} & \multicolumn{4}{|c|}{ Percent Reduction in Total Curve Crashes } \\
\cline { 2 - 5 } & $1: 4$ & $1: 5$ & $1: 6$ & $1: 7$ or flatter \\
\hline $1: 2$ & 6 & 9 & 12 & 15 \\
\hline $1: 3$ & 5 & 8 & 11 & 15 \\
\hline $1: 4$ & - & 3 & 7 & 11 \\
\hline $1: 5$ & - & - & 3 & 8 \\
\hline $1: 6$ & - & - & - & 5 \\
\hline
\end{tabular}

Source: [Zegeer et al., 1991]

A descriptive analysis of the distribution of curve sections with inadequate recovery distance and side slopes and the variation of such defective situations with crash rates could not be carried out due to lack of data. The Safety Management Unit at INDOT is currently in the process of acquiring field data from which it may be possible to obtain such information for curve sections. With such data, a future study could therefore examine the relationships between crash rates and recovery distance and side-slope conditions on Indiana's rural two-lane system.

\subsubsection{Distribution of Horizontal Curves Indiana's Rural Two-lane Highways, by Functional Class and Region}

The distribution of average horizontal curve conditions by functional class and region were investigated as part of the present study. Horizontal curve conditions may be expressed in any of the following ways:

- Average number of curves per mile

- Average radius per curve per mile 
- Average curvature per curve per mile

- Average length per curve per mile

For purposes of the descriptive analysis, the horizontal curve density (number of horizontal curves per mile) was used. The horizontal curve alignment information was available for approximately half of the road sections in the database hence analysis involving the horizontal alignment variable was computed for only the sections with curve information. The following ranges were used for the categorization of the section horizontal curve density.

$\begin{array}{lrr}\text { Very Small } & = & 0<H C D \leq 0.5 \\ \text { Small } & = & 0.5<H C D \leq 1 \\ \text { Medium } & = & 1<H C D \leq 2.5 \\ \text { Large } & = & H C D>2.5\end{array}$

Figures 6-14 and 6-15 present the distribution of curve sections by functional class and region. Curve data are still being collected for the road sections. As such, the observations from these figures may not be conclusive. The correlation matrix from the Indiana data (shown as Table 6-11) suggests that increasing number of curves per mile is associated with higher number of crashes, which is expected.

Table 6-11: Correlation Matrix for Horizontal Curves and Crash Occurrence

\begin{tabular}{|c|c|c|c|c|c|}
\hline & $\begin{array}{c}\text { Fatal Crash } \\
\text { Rate }\end{array}$ & $\begin{array}{c}\text { Injury } \\
\text { Crash Rate }\end{array}$ & $\begin{array}{c}\text { PDO } \\
\text { Crash Rate }\end{array}$ & $\begin{array}{c}\text { Fatal or Injury } \\
\text { Crash Rate }\end{array}$ & $\begin{array}{c}\text { Total Crash } \\
\text { Rate }\end{array}$ \\
\hline $\begin{array}{c}\text { Horizontal Curve Densities } \\
\text { at Rural Two-Lane State } \\
\text { Highways in Indiana }\end{array}$ & 0.0139 & 0.1633 & 0.0813 & 0.1562 & 0.1078 \\
\hline
\end{tabular}



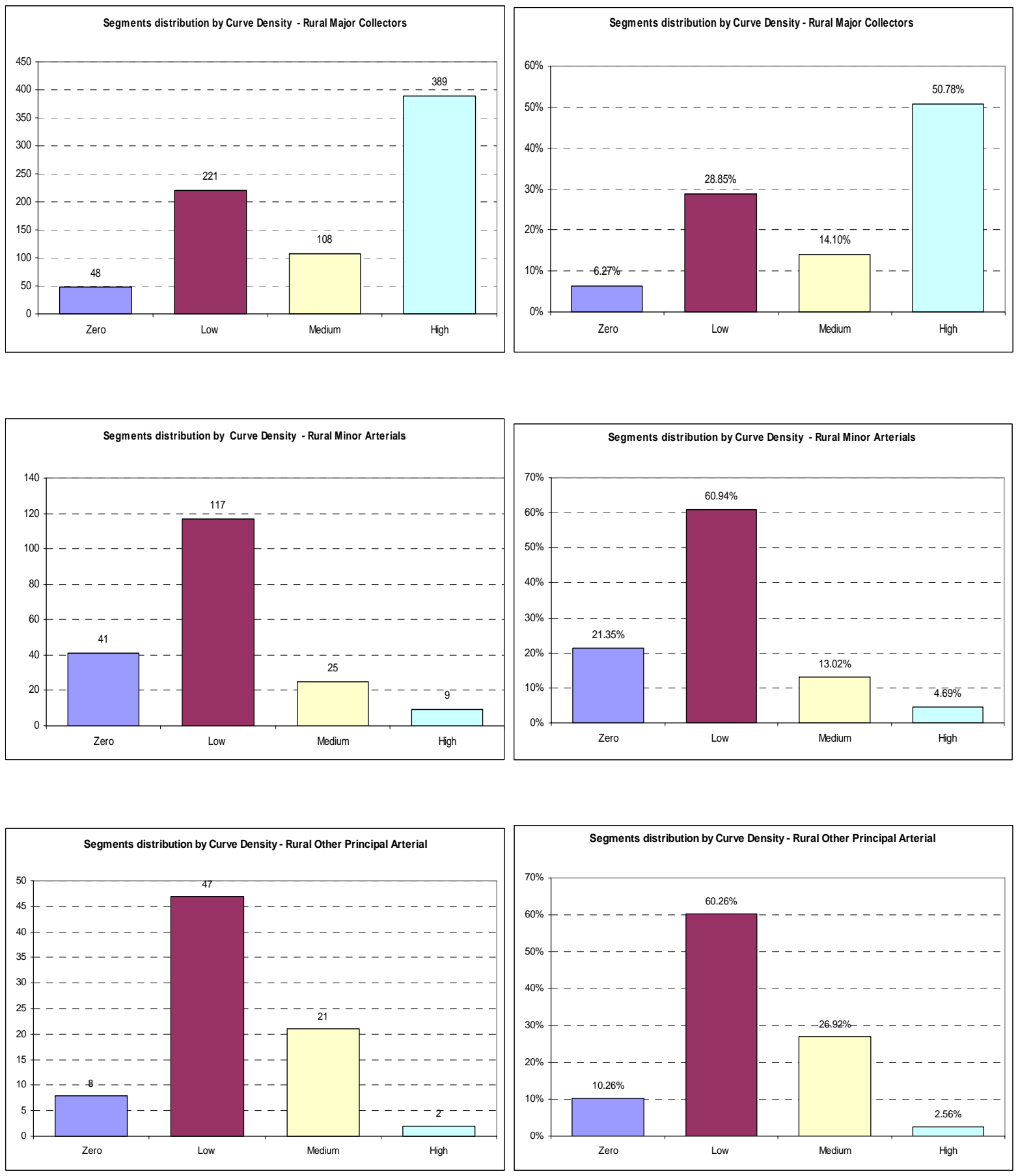

Figure 6-14: Distribution of Horizontal Curve Density at Rural Two-Lane State Highways, by Functional Class 

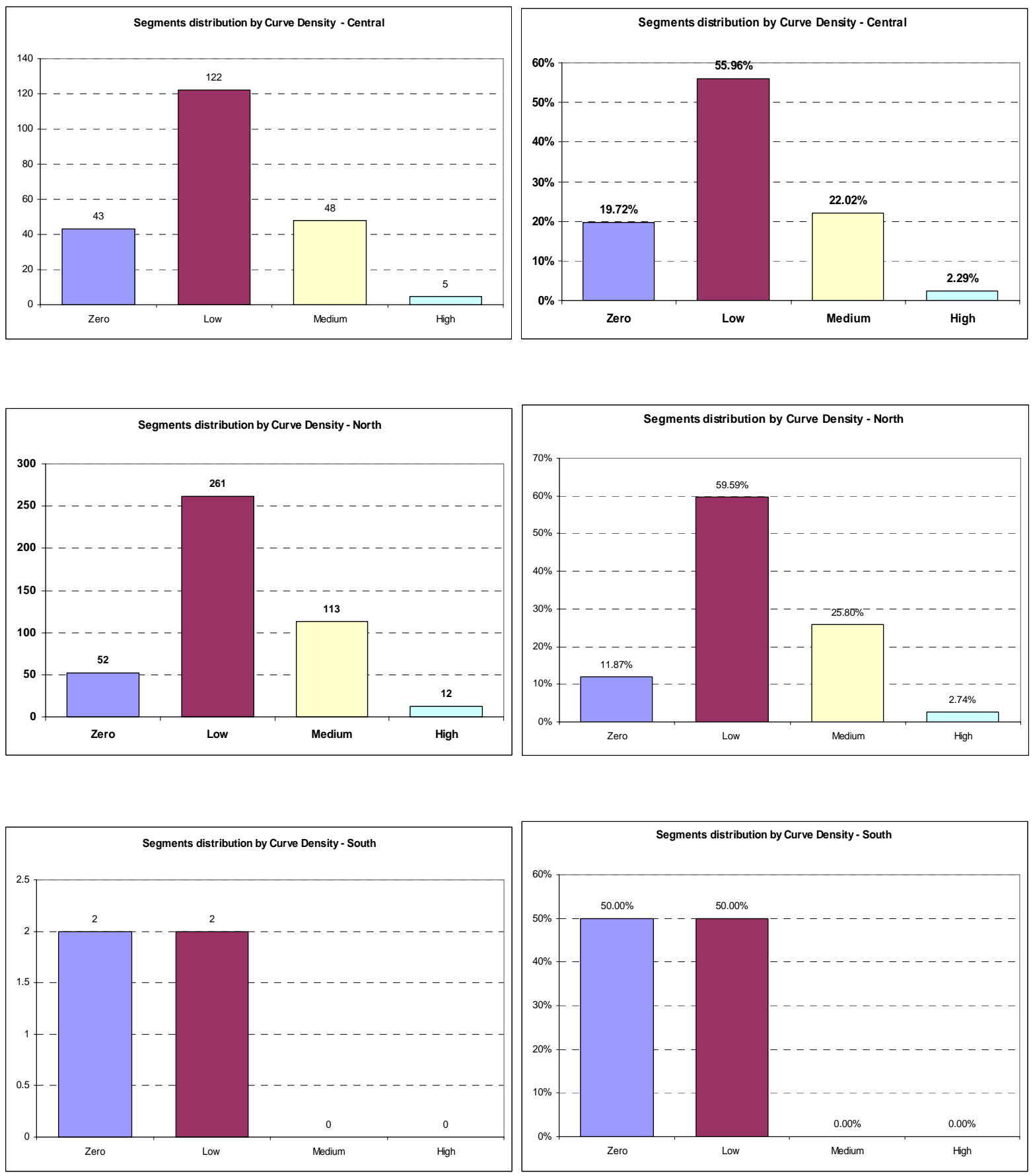

Figure 6-15: Distribution of Horizontal Curve Density at Rural Two-Lane State Highways, by Region 


\subsubsection{Variation of Crash Rates with Horizontal Curve Density by Functional Class}

Using data on geometric alignment obtained from INDOT, a preliminary descriptive analysis of the variation of crash rates with horizontal curvature was carried out (Figure 6-16). The ordinate axis shows the average annual crash rate per vehicle-miles-of-travel. The horizontal curve densities were grouped in bins of: zero or very small, small, medium, and large.

From "small" to "large" horizontal curve densities, irrespective of crash severity type or road functional class, increasing horizontal curve density is generally seen to be positively related to crash occurrence. This is consistent with expectation. The relationship seemed to be most obvious for fatal crashes at rural collectors and principal arterials.

From "very small" to "small" densities, however, crashes seem to decrease with increasing density - a trends that seems to reflect the homeostatic effects of this geometric feature.
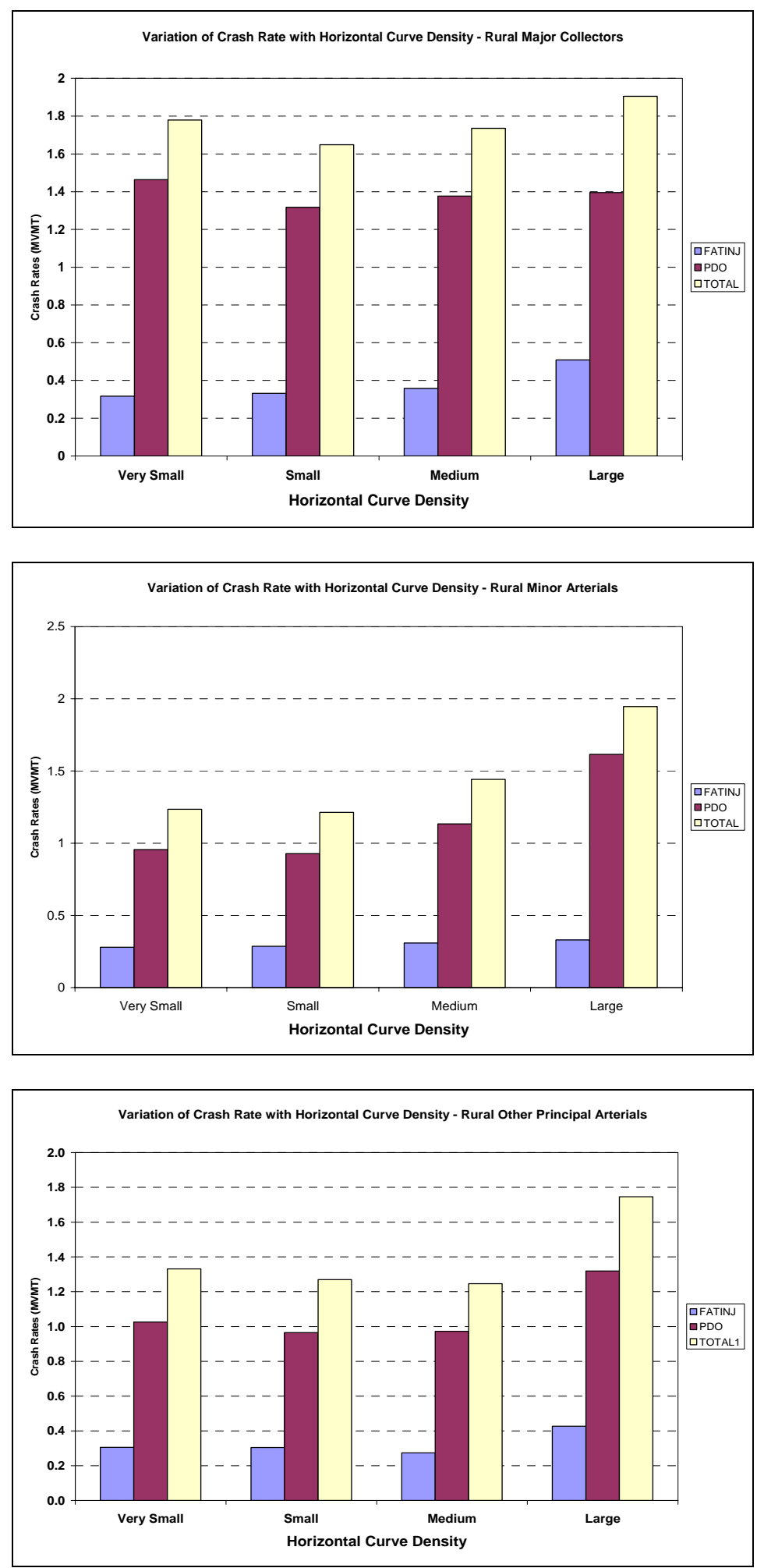

Figure 6-16: Variation of Crash Rates with Horizontal Curve Density by Functional Class 


\subsubsection{Vertical Alignment}

Vertical alignment consists of straight grades, crests and sags. A major consideration in the design of crest vertical curves is the difference between the grades and the stopping sight distance required for that class of roadway. Stopping sight distance is the sight distance available on a roadway which below-average operators traveling at or near the design speed would need to come to a complete stop before reaching a stationary object in their path. With respect to crest vertical curves, the sight distances for design are determined such that drivers are able to see over the top of the hill and therefore discern objects at the other side. For sag vertical curves, the design sight distances are determined such that drivers can see at night from vehicle headlights.

The final vertical alignment constructed for a highway section is the end result of a typically laborious evaluation of the benefits and costs associated with alternative route locations at the planning stage, with due consideration given to existing terrain, safety, and construction haulage and cost, among others. Grades or straight vertical sections are designed to be steep enough to allow for longitudinal drainage, but not so steep as to pose a danger to vehicles through inadvertent increased speed downhill (and conversely, difficulty of climbing uphill and posing safety risks where there are inadequate passing opportunities) [INDOT, 2000]. Clearly, safety consideration play a pivotal role in the vertical design of roadways, and therefore design features need to be considered in any study that seeks to investigate factors affecting highway safety.

Table 6-12 shows that downgrade crashes are more frequent and result in higher percentages of injuries and fatalities compared to upgrade crashes. Also, injury and fatality rates on vertical curves are higher than those at level or upgrade locations. The crash rates for downgrades are 63 percent higher than upgrades, all else being the same [Brinkmen and Perchonk, 1979].

Table 6-12: Crash Frequency and Severity by Vertical Alignment

\begin{tabular}{|l|c|c|c|c|}
\hline $\begin{array}{l}\text { Nature of Vertical } \\
\text { Alignment }\end{array}$ & $\begin{array}{c}\text { Observed Number } \\
\text { of Accidents }\end{array}$ & $\begin{array}{c}\text { Percent of Total } \\
\text { Accidents }\end{array}$ & $\begin{array}{c}\text { Percent } \\
\text { Injured }\end{array}$ & Percent Fatal \\
\hline Level & 2001 & 34.6 & 53.6 & 4.7 \\
\hline Upgrade & 943 & 16.3 & 55.6 & 3.9 \\
\hline Downgrade & 1533 & 26.5 & 58.4 & 5.1 \\
\hline Up on Crest & 373 & 6.5 & 59.5 & 6.0 \\
\hline Down on Crest & 461 & 8.0 & 62.6 & 5.9 \\
\hline Up on Sag & 258 & 4.5 & 57.8 & 6.3 \\
\hline $\begin{array}{l}\text { Down on Sag } \\
\text { Total Known }\end{array}$ & 211 & 3.7 & 61.7 & 6.8 \\
\hline $\begin{array}{l}\text { Tnknown } \\
\text { Sotal }\end{array}$ & 5780 & 100 & & \\
\hline
\end{tabular}


Adequate vertical curve sight distances can help lower crash rates (Plate 6-3). St. John and Kobett [1978] investigated the safety effects of long, steep grades on rural two-lane highways using traffic speed distributions generated with computer simulation, using the deviations from the mean speeds to estimate expected crash rates. With a truck percentage of $20 \%$ (it was assumed that such trucks were low performance), the crash involvement rate increases from $175 \%$ of passenger car rates for 4 percent grades, to $250 \%$ of passenger cars for $8 \%$ grades. At areas of rolling terrain (presumably similar to that of southern Indiana), the crash rates are expected to be slightly higher due to the presence of recreational vehicles and trucks. On steep downgrades (greater than 4\%) trucks using crawl speeds to maintain control tend to increase crash rates.

It has often been hypothesized that elements of vertical alignment that cause crashes include steepness, length of grade, and vertical curve design. Geometric countermeasures for vertical alignment include minimizing the effects of slower moving vehicles by providing opportunities to pass, and increasing the sight distance on vertical curves, or minimizing the hazards on vertical curves. Miaou [1998] developed accident modification factors to explain the effect of vertical grade on crash frequency on rural two-lane roads. The IHSDM utilizes the accident modification factors shown in Table 6-13.

Table 6-13: Accident Modification Factors for Grade of Roadway Sections

\begin{tabular}{|l|c|c|c|c|c|}
\hline \multirow{2}{*}{} & \multicolumn{5}{|c|}{ Grade (\%) } \\
\cline { 2 - 6 } & 0 & 2 & 4 & 6 & 8 \\
\hline AMF & 1.00 & 1.03 & 1.07 & 1.10 & 1.14 \\
\hline
\end{tabular}

Source: Harwood et al. [1998]

Regarding the impacts of vertical curves on crash experience, Olson [1984] determined that vertical curves with stopping sight distances less than $311.5 \mathrm{ft}$ had more crashes than vertical curves with longer stopping sight distances. In a similar study, Fambro et al., [1989] determined that stopping sight distance ranging from 328 to $426.5 \mathrm{ft}$ did not affect crash rates unless an intersection was within the limited sight distance section. In 1997, Fambro et al. [1997] found that accident rates on rural two-lane highways with limited stopping sight distance are similar to the crash rates on all two-lane highways. Fitzpatrick et al [2000] reviewed such findings and concluded that for the range of conditions studied, limited stopping sight distances did not appear to cause a safety problem and made the following recommendations: 
- Many design criteria are based on parameters associated with the interaction between drivers, vehicles, and roadways. As such, the resultant design criteria should be greater than or equal to the minimum requirements for safety,

- A minimum stopping sight distance for safe operations and $56 \mathrm{mph}$ speed lies within the range 311.7 - $361 \mathrm{ft}$, which is less than that in AASHTO's Green Book. As such, the researchers concluded that the AASHTO values are still acceptable for design but could be increased under special circumstances,

- As there seem to be little or no safety benefits from providing sight distances longer than 360 $\mathrm{ft}$ (when other hazards are not present), improvements other than lengthening a limited stopping sight distance of a crest vertical curve may be more cost-effective.

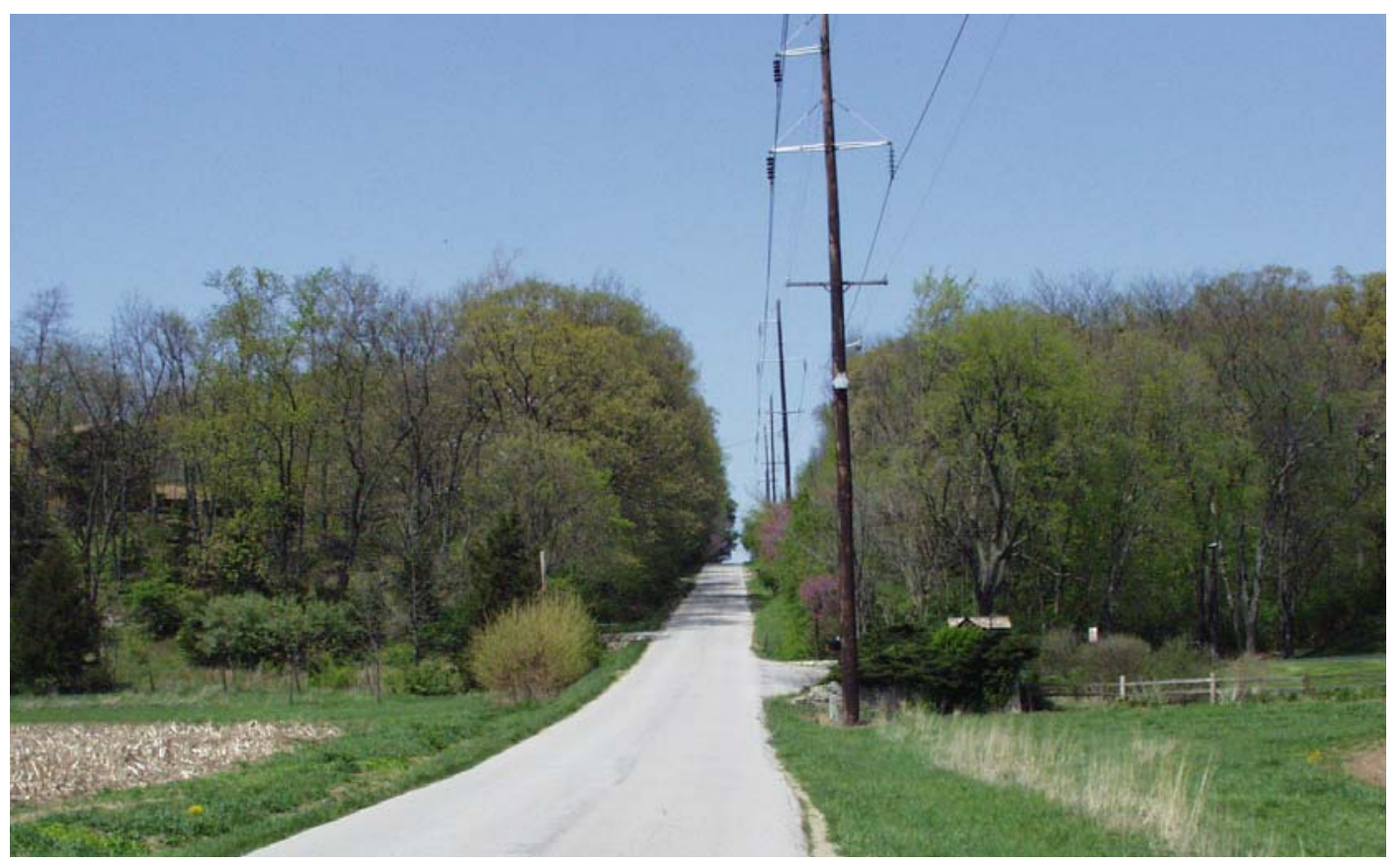

Plate 6-3: Inadequate Vertical Curve Sight Distance May be Associated with High Crash Rates 


\subsubsection{Distribution of Vertical Curves on Rural Two-lane Highways in Indiana, by Functional Class and Region}

The distribution of average vertical curve conditions by functional class and region were investigated as part of the present study. Like horizontal curves, vertical curve conditions may be measured in any of several ways, such as:

- Average number of vertical curves per mile

- Average grade per curve per mile

For purposes of the descriptive analysis, the vertical curve density, VCD, (number of vertical curves per mile) was used. The following ranges were used for categorizing the sections on the basis of their vertical curve density:

$\begin{array}{llr}\text { Very Small } & = & 0<V C D \leq 0.5 \\ \text { Small } & = & 0.5<V C D \leq 1 \\ \text { Medium } & = & 1<V C D \leq 2.5 \\ \text { Large } & = & V C D>2.5\end{array}$

The correlation matrix for vertical curve density and crashes was similar to that obtained for horizontal curves, as shown in Table 6-14. As such, it is clear that the data for Indiana's rural two-lane roads shows that increasing vertical curve density is associated with increasing crash occurrence. This is consistent with intuition.

Table 6-14: Correlation Matrix for Vertical Curves and Crash Occurrence

\begin{tabular}{|c|c|c|c|c|c|}
\hline & $\begin{array}{c}\text { Fatal Crash } \\
\text { Rate }\end{array}$ & $\begin{array}{c}\text { Injury } \\
\text { Crash Rate }\end{array}$ & $\begin{array}{c}\text { PDO } \\
\text { Crash Rate }\end{array}$ & $\begin{array}{c}\text { Fatal or Injury } \\
\text { Crash Rate }\end{array}$ & $\begin{array}{c}\text { Total Crash } \\
\text { Rate }\end{array}$ \\
\hline $\begin{array}{c}\text { Vertical Curve Densities } \\
\text { at Rural Two-Lane State } \\
\text { Highways in Indiana }\end{array}$ & 0.0509 & 0.1866 & 0.1548 & 0.1839 & 0.1797 \\
\hline
\end{tabular}




\subsubsection{Variation of Crash Rates with Vertical Curve Density by Functional Class}

Using data on geometric alignment obtained from the Indiana HPMS datafile for HERS Applications, a preliminary descriptive analysis of the variation of crash rates with vertical curvature was carried out. The ordinate axis shows the average annual crash rate per vehicle-miles-of-travel. The vertical curve densities were grouped in bins of: very small, small, medium, and large (Figure 6-17).

Besides a small drop in crash rate from "very small" to "small" vertical curve density for rural minor collectors and rural other principal arterials, increasing vertical curvature is generally seen to be positively related to crash occurrence for the various crash types. In other words, higher incidence of vertical curvature seems to be generally associated with higher crashes. This is consistent with expectation.
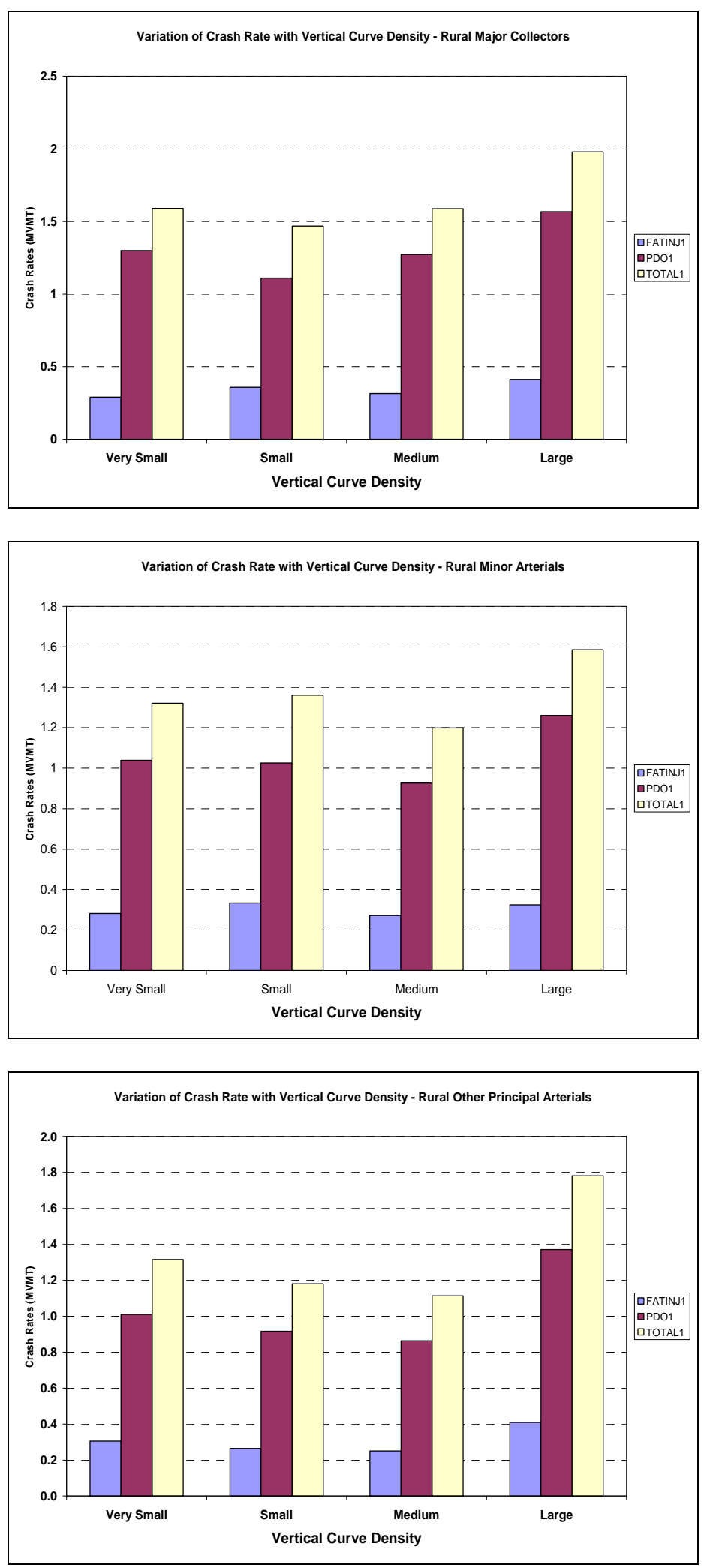

Figure 6-17: Variation of Crash Rates with Vertical Curve Density by Functional Class 


\subsubsection{Centerline Rumble Strips}

Rural two-lane roads typically lack physical elements to separate opposing traffic streams, such as wide or raised medians. As a result, a major crash problem on such roads involves vehicles crossing the centerline and either side-swapping other vehicles or striking opposing vehicles head-on. The risk of such crashes could be reduced by roadway widening or median construction. However, such countermeasures are expensive and are generally applied only at high priority localized areas. Alternative, wider-scoped countermeasures are continually being investigated for implementation.

In an article in Indiana LTAP's Newsletter The Pothole Gazette, for instance, Retting [2003] advocated the use of centerline rumble strips as a more widely applicable countermeasure to reduce the incidence of such centerline incursions. Rumble strips are raised or grooved patterns impressed on the pavement surface or shoulder. The passage of vehicle tires over rumble strips produces a rumbling sound that causes the vehicle to vibrate. Rumble strips are typically used to provide drivers with an audible and tactile warning that (i) they are approaching an upcoming change in the roadway, such as a hazardous horizontal curve, and (ii) their vehicle is leaving their share of the carriageway either to the right (towards the shoulder and onto the roadside) or to the left (across the centerline in the face of oncoming traffic). Rumble strips may be milled, rolled, formed, or raised. This section focuses on rumble strip applications on the carriageway where such facility is provided on the centerline [Fitzpatrick et al., 2000], as shown in Plate 6-4). Retting [2003] reported on a before-after study was undertaken at almost 100 sites located at seven states to investigate the effectiveness of centerline rumble strips, and it was found that with the use of such technology, there was a $14 \%$ reduction in all crashes, and a 15\% reduction in injury crashes. Furthermore, head-on and opposing direction side-swipe crashes were reduced by $21 \%$, and for such crashed that involved injury, the reduction was $25 \%$ Given such demonstrated cost-effectiveness of centerline rumble stripping, it is obvious that this countermeasure has great potential in reducing crashes on rural two-lane highways in Indiana and elsewhere.

At the present time, there is no indication that Indiana's Roadway Inventory File, HPMS datafile, or other data sources have data showing which rural two-lane sections have rumble strips and where such strips may be located. As such, the effectiveness of this countermeasure could not be

investigated with Indiana data in the present study. With currently ongoing data collection efforts by INDOT's safety management unit, it is expected that such data may be available in the near future, and the needed analysis of centerline impacts on crashes can be carried out. 


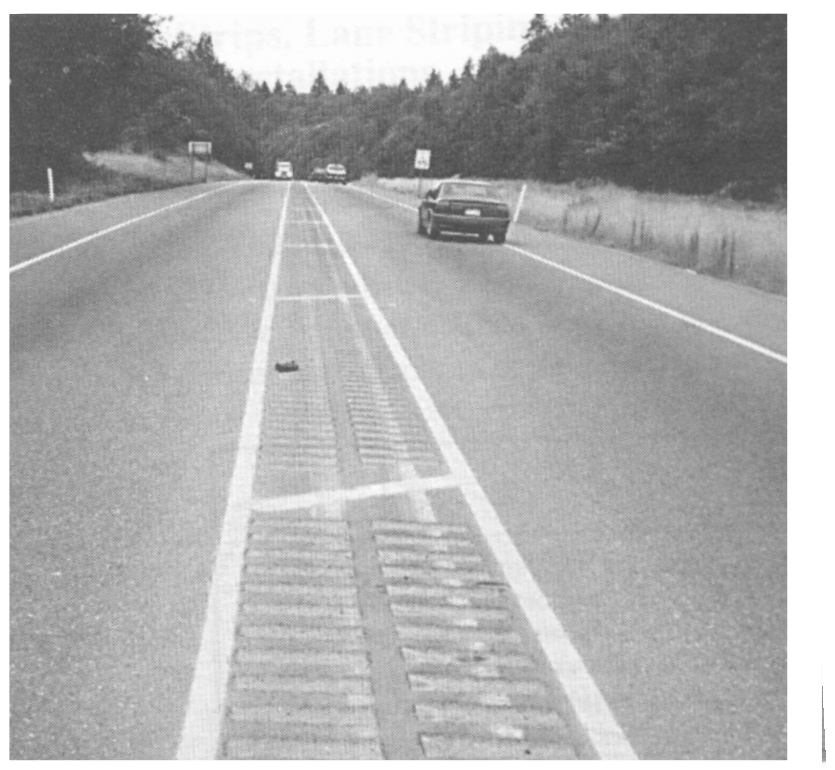

Plate 6-4: Centerline Rumble Strips [Fitzpatrick et al., 2000]

\subsection{SHOULDER FACTORS}

Shoulders are the portion of the roadway immediately adjacent to, and outside of, the travel lanes. Shoulders are typically designed and intended to accommodate occasional use by vehicles but not continual travel. Part or all of the shoulder may be paved. The total roadway width comprises the lane width plus shoulder width (plus median width, if any).

\subsubsection{Shoulder Type and Width}

The shoulder is that part of the roadway that is immediately adjacent to but outside of the carriageway. As mentioned above, shoulders are designed for use by vehicular traffic for temporary stops, or occasional travel. The shoulder width is measured as the distance between the pavement edges to the start of the side-slope. A shoulder may be fully paved, partially paved, or unpaved. Also, a shoulder may be at the same level as the carriageway or may be at a lower elevation than the carriageway (indicting a shoulder “drop-off” defect). Countermeasures for shoulder problems include:

- widening the shoulder,

- paving the shoulder, and 
- raising the shoulder to carriageway level.

Improving the shoulder typically yields a structurally stable shoulder and also that provides adequate surface friction to help control or stop errant vehicles. Also, widening a shoulder provides additional recovery area for vehicles before they leave the roadway and also makes available additional space for vehicles to use if their carriageway is being encroached by an oncoming vehicle. A number of studies have been carried out to express crash experience as a function of shoulder width, shoulder type (paved vs. unpaved), and shoulder drop-off status.

The 1987 FHWA by Zegeer et al. also investigated the effects of shoulder type and width on crash experience at the rural two-lane highways, and also found that accident types related to shoulder type and width included run-off-the-road, head-on, same-direction-sideswipe, and opposite-directionsideswipe. That study also estimated the percent reduction in crash frequency as a result of increasing the widths of paved and unpaved shoulders (Table 6-15). The table shows that for rural two-lane roads, widening a $2 \mathrm{ft}$ gravel shoulder to $8 \mathrm{ft}$, for example, will reduce "related" crashes by $35 \%$, all other factors remaining the same. The research by Zegeer et al. has been deemed the most reliable study that assessed the effect of shoulder width on safety for rural two lane highways with ADTs over 2,000 vpd [Harwood et al., 1998] and was subsequently adopted by the panel of experts that convened in Washington in September 1997 to resolve the issue for the IHSDM. Tables 6-16 and 617 illustrate the recommendations of that panel for the effect of shoulder width and type, respectively, on safety. Fitzpatrick et al [2000] state that interpolation within the values is encouraged, but extrapolation beyond their limits is not.

Table 6-15: Effectiveness of Shoulder Type and Widening with Respect to "Related "Crashes

\begin{tabular}{|c|c|c|}
\hline \multirow{2}{*}{ Amount of Lane Widening (ft) } & \multicolumn{2}{|c|}{$\%$ Reduction in Crashes } \\
\cline { 2 - 3 } & Paved & Unpaved \\
\hline 2 & 16 & 13 \\
\hline 4 & 29 & 35 \\
\hline 6 & 40 & 43 \\
\hline 8 & 49 & 25 \\
\hline
\end{tabular}

Note: These values are only for rural two-lane roads.

Source: [Zegeer et al., 1987 ] 
Table 6-16: Accident Modification Factors for Shoulder Width

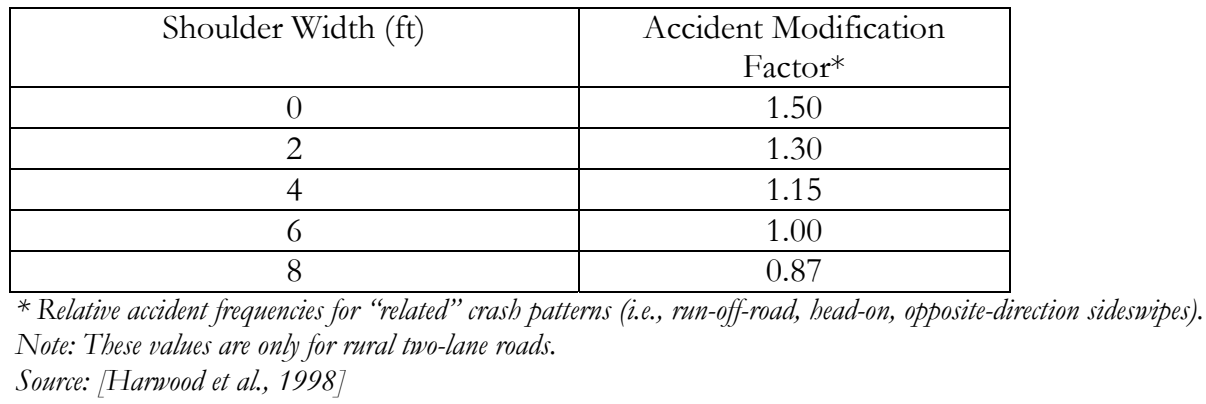

Table 6-17: Accident Modification Factors for Shoulder Types

\begin{tabular}{|c|c|c|c|c|c|c|c|c|}
\hline \multirow{3}{*}{ Shoulder Type } & \multicolumn{8}{|c|}{ Accident Modification Factor* } \\
\hline & \multicolumn{8}{|c|}{ Shoulder Width (ft) } \\
\hline & 0 & 1 & 2 & 3 & 4 & 6 & 8 & 10 \\
\hline Paved & 1.00 & 1.00 & 1.00 & 1.00 & 1.00 & 1.00 & 1.00 & 1.00 \\
\hline Gravel & 1.00 & 1.00 & 1.00 & 1.01 & 1.01 & 1.02 & 1.02 & 1.03 \\
\hline Composite & 1.00 & 1.01 & 1.02 & 1.02 & 1.03 & 1.04 & 1.06 & 1.07 \\
\hline Turf & 1.00 & 1.01 & 1.03 & 1.04 & 1.05 & 1.08 & 1.11 & 1.14 \\
\hline
\end{tabular}

* Relative accident frequencies for "related" crash patterns (i.e., run-off-road, head-on, opposite-direction sideswipes).

Note: These values are only for rural two-lane roads. Source: [Harwood et al., 1998]

Zegeer and Deacon (1987) analyzed data covering a wide range of lane- and shoulder-width and shoulder type combinations, and provided a critique of over 30 years of literature to summarize safety relationships between geometric characteristics and crashes. Among their conclusions was that most studies summarized that lane and shoulder conditions have a greater effect on "run off the road (ROR)" and "other direction (OD)" crashes compared to rear-end and angle crashes. The crashes decrease with increasing shoulder width and lane width. It was observed that lane width has larger impact on crash rates than shoulder-width. Also, Abdel and Radwan (2000) estimated a negative binomial regression model for an arterial in Florida and concluded that improvements in horizontal curvature, shoulder, lane and median widths reduce crashes.

In what was probably the most interesting literature reviewed for shoulder widths, revealing relationships were shown between shoulder improvement and crash rate (Ogden, 1997). Crash data from selected two-lane rural road sections were analyzed and it was found that paving a highway shoulder led to a statistically significant (40\%) reduction of crash rates expressed as number of crashes per VMT. Ogden concluded that safety benefits of paved shoulders arise from increased recovery space and consequently reductions in single-vehicle run-off-the road crashes. Such crashes 
are typically due to loss of control when a vehicle strays onto an unpaved shoulder. There are also benefits of multi-vehicle opposite-direction crashes and increased avoidance space. The study included a cost-effectiveness analysis which determined the benefits (accident reductions) corresponding to each unit of shoulder paving expenditure. The study also determined the breakeven point (vehicle exposure at which it becomes economically worthwhile to pave shoulders).

In an article titled "What's that Extra Two Feet of Lane or Shoulder Width Worth?”, Sunley [1990] cited findings from past studies, stating that the first and second ft. of lane widening correspond to $12 \%$ and $23 \%$ reductions, respectively, in crashes related to these deficiencies. With regard to shoulders, Sunley stated that an additional foot and two feet correspond to $16 \%$ and 13\% reduction, respectively, in crashes. Such findings led to improved policies geared at enhancing safety at local road agencies in Illinois.

Zegeer et al. [1981] investigated the interrelated effects of various combinations of lane and shoulder widths, and found that of the various crash types, only ROR and OD crashes were significantly influenced by lane and shoulder width. They found that rates of these two crash types generally decrease as lane and shoulder widths increase as seen in Figure 6-18. Commenting on the Zegeer et al. study, Hauer [2000 (2)] argued that the effect of shoulder widening on all crashes, rather than only ROR and OD crashes, should be the focus.

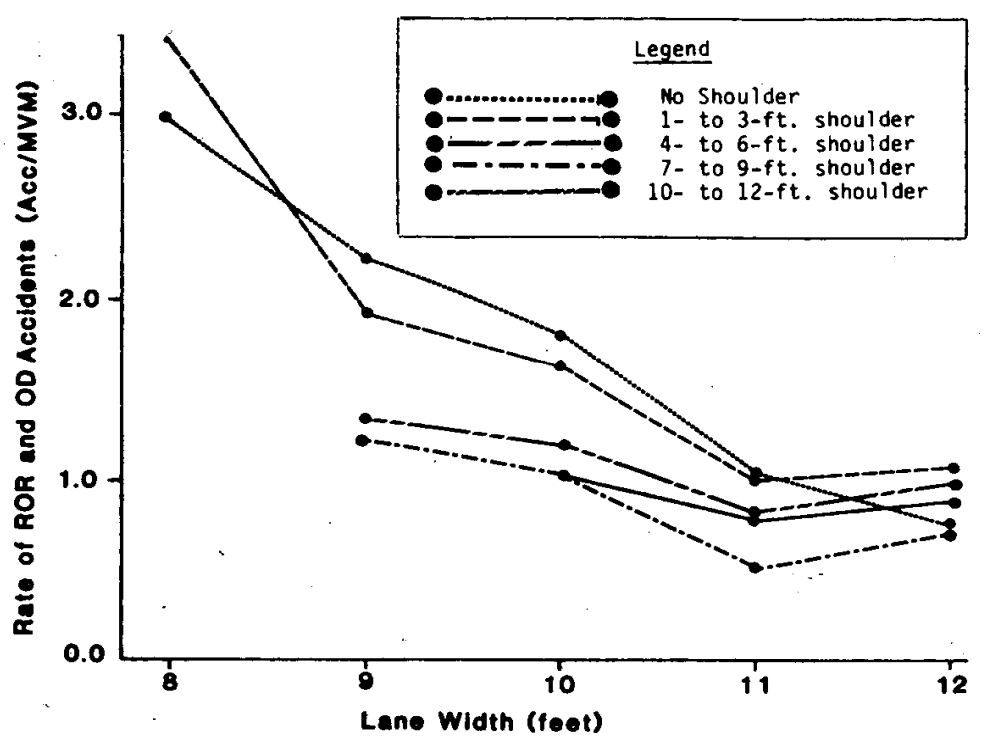

Figure 6-18: Relationship between Lane Width and Crashes [Zegeer et al., 1981]

Using single vehicle crash data, traffic data, and roadway inventory from approximately 600 2-lane rural road sections in Alabama, Michigan, and Washington, Miaou [1998] determined that 
increasing shoulder width by one ft decreases the number of single vehicle run-off-the-road crashes by approximately $9 \%$. In a similar study for rural two-lane roads in North Carolina and Washington, Stewart and Council fitted models that expressed annual crashes per mile as exponential functions of traffic volume. Separate models were built for relatively narrow $(6 \mathrm{ft})$ and relatively wide $(8 \mathrm{ft})$ shoulders. It was shown that increasing the shoulder width by $1 \mathrm{ft}$ can lead to a $5-10 \%$ reduction in crashes at sections where traffic volume exceeds 5,000 per day.

Vogt and Bared used three-year data from Minnesota and Washington to estimate models for rural two-lane roads, giving due cognizance to traffic volume, roadway width, roadside hazard rating, driveway density, grade, and curvature. It was found that a unit increase in shoulder width had significant impacts on crash reduction.

\subsubsection{Distribution of Shoulder Widths at Rural Two-lane Highways in Indiana, by Functional Class and Region}

From the dataset prepared for the present study, the distribution of shoulder widths at Indiana's rural two-lane state highways were investigated. This was done across functional classes (Figure 6-19) and regions (Figure 6-20). For sections having segments with different shoulder widths (SW), the weighted average of the shoulder width for the entire section was calculated and used for the analysis. For purposes of the preliminary descriptive analysis, the following ranges were used categorizing shoulder widths.

$\begin{array}{llr}\text { Very Small } & = & 0<S W \leq 2 \mathrm{ft} \\ \text { Small } & = & 2<S W \leq 4 \mathrm{ft} \\ \text { Medium } & = & 4<S W \leq 8 \mathrm{ft} \\ \text { Large } & = & S W>8 \mathrm{ft}\end{array}$

The preliminary descriptive analysis showed that shoulder width generally ranges from zero to over $10 \mathrm{ft}$. As shown in Figure 6-19, for all three road classes, the most frequent shoulder width range is 2-4 ft. However, a rather large fraction (over 25\%) of rural collectors have shoulder widths of less than $2 \mathrm{ft}$., while only approximately $8 \%$ of rural minor and principal arterial lanes have shoulder widths in that category. This shows that rural minor and principal arterials in Indiana have shoulder geometric standards that are similar to each other but superior to that of rural major collectors.

From the perspective of regional variations in shoulder widths (Figure 6-20), it is seen that shoulder widths generally decrease from north to southern Indiana. Most northern road shoulders have a shoulder width between $4-6 \mathrm{ft}$, while most in the south have 2-4 ft. Also, almost 30\% of sections in the south have shoulder widths less than $2 \mathrm{ft}$, while only approximately $9 \%$ of northern 
sections fall in that category. The case for central pavements lies in between. Such variations may be attributed to the larger number of lower functional class roads in the south.
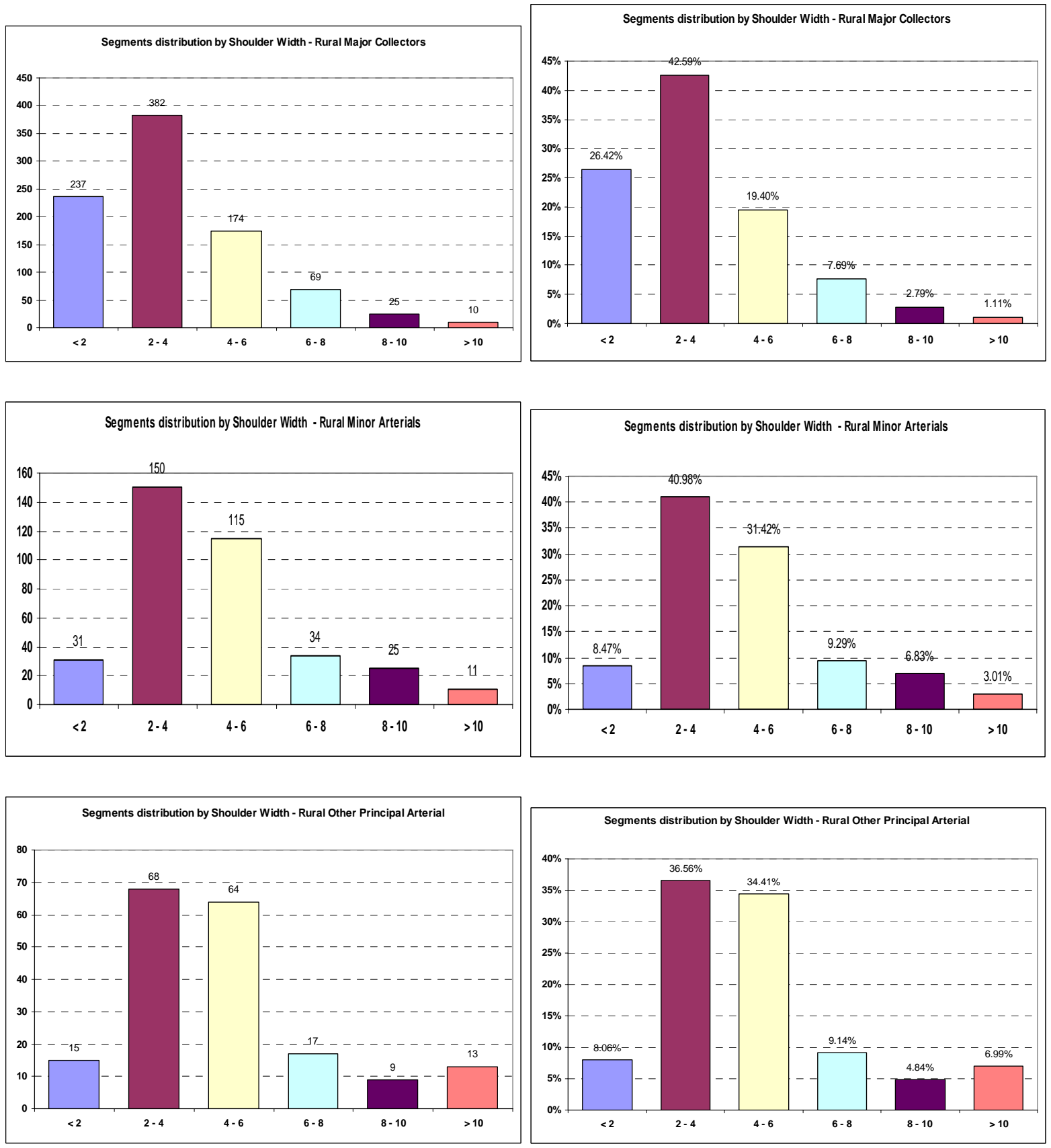

Figure 6-19: Shoulder Width at Distribution at Rural Two-lane State Highways in Indiana, by Functional Class 

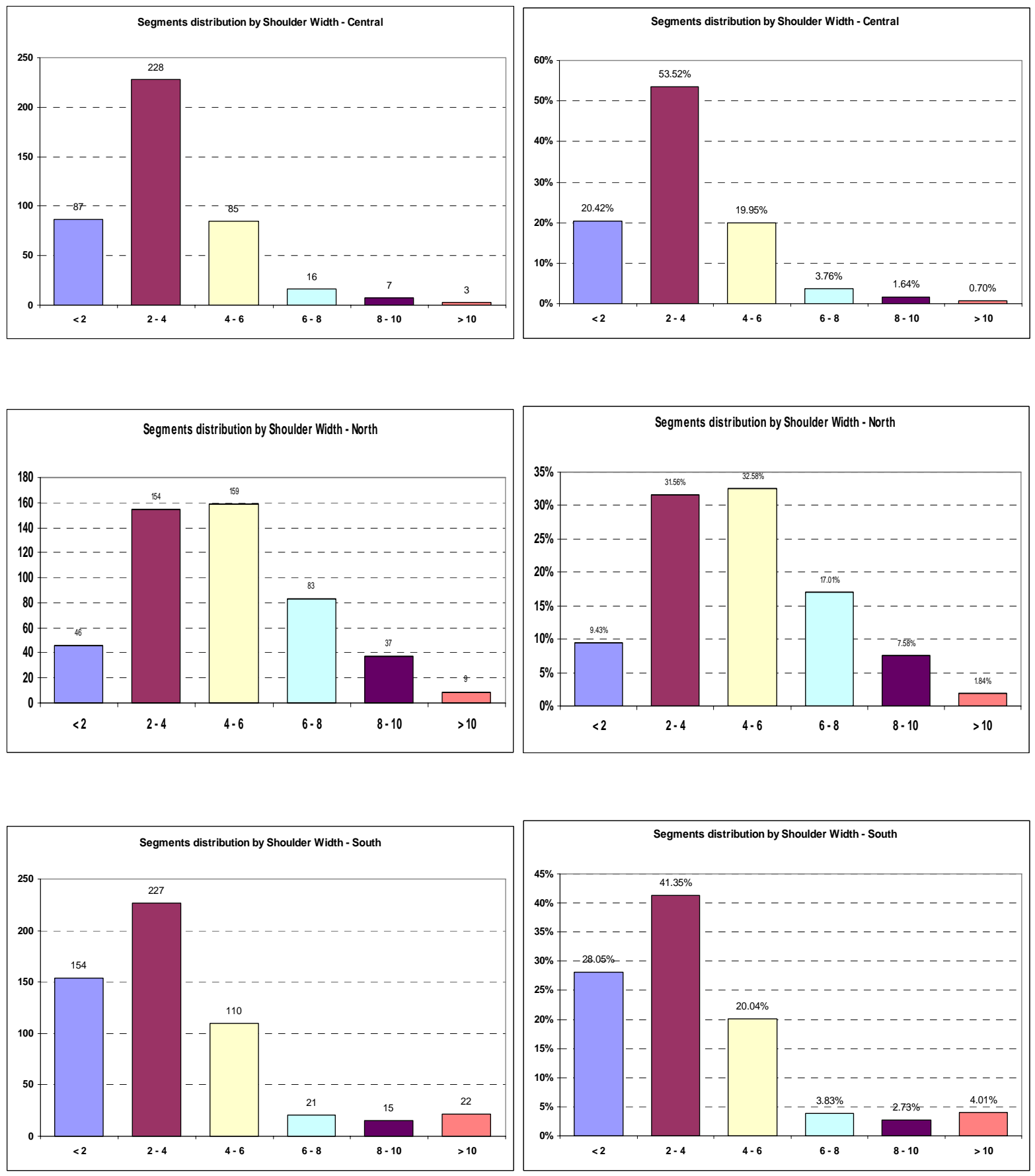

Figure 6-20: Shoulder Width Distribution at Rural Two-lane State Highways in Indiana, by Region 


\subsubsection{Variation of Crash Rates with Shoulder Width by Functional Class}

A preliminary descriptive analysis of the variation of crash rates with shoulder widths was carried out using 1997-2000 data from Indiana. The shoulder widths were grouped in bins of: very small, small, medium, and large. The results (Figure 6-21) clearly showed that increasing shoulder widths generally leads to decreasing crashes. Absence of a shoulder denies any refuge for vehicles facing imminent collision or sideswiping situations with opposing or samedirection vehicles and increases chances of crashes.

This trend was the same for all crashes, casualty crashes (fatal+injury), and property-damage-only crashes. Also, the results were somewhat consistent for all three functional classes of rural two-lane highways (principal, minor arterials, and major collectors), particularly for the rural arterial system.

It is also seen that irrespective of crash severity type, crash rates seem to decrease as the level of functional class "increases" from collectors to principal arterials. For a given shoulder width: from major collectors to minor arterials, there is generally a small decrease in crash rates: and from minor arterials to principal arterials, there is generally a large drop in crash rates.
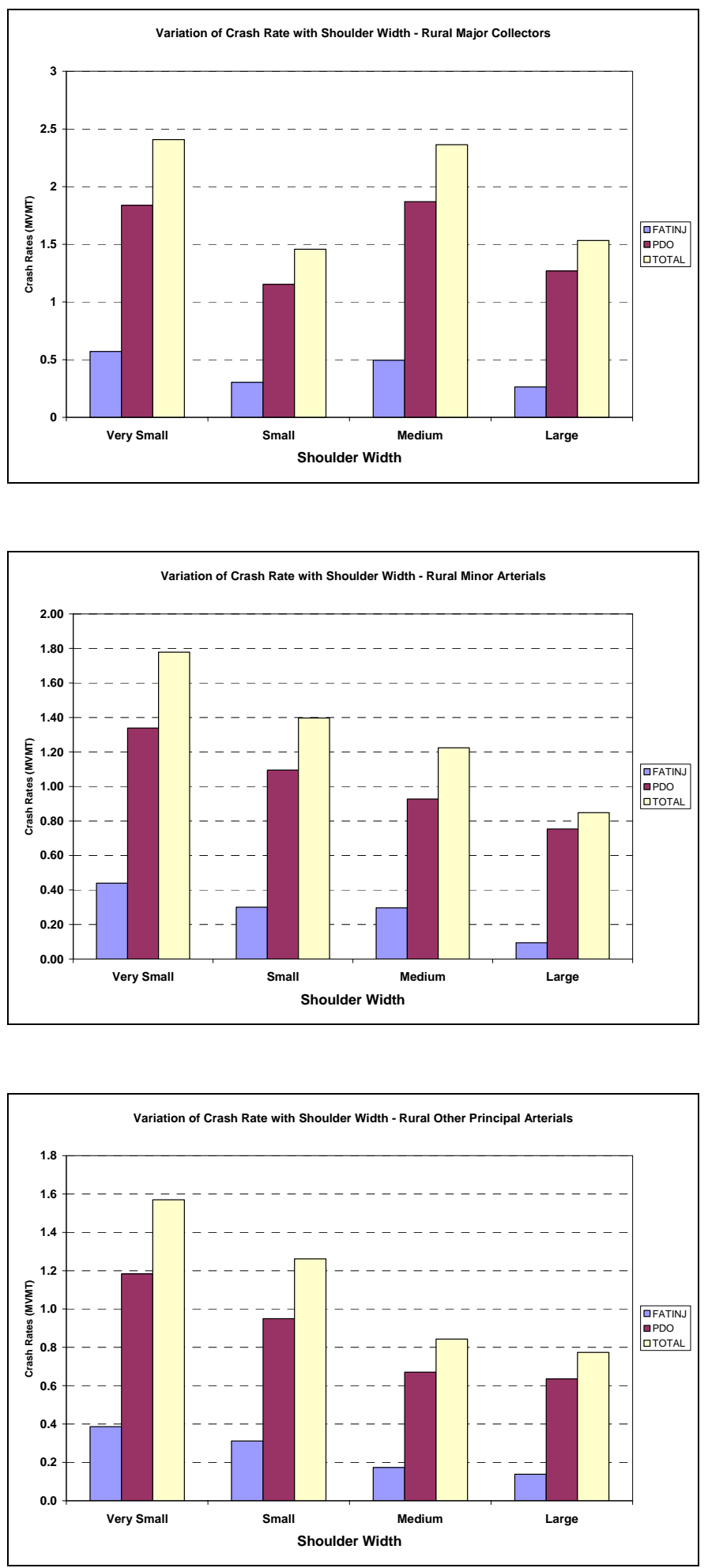

Figure 6-21: Variation of Crash Rates with Shoulder Width by Crash Severity and Functional Class 


\subsubsection{Distribution of Shoulder Type at Rural Two-lane Highways in Indiana, by Functional Class and Region}

Using data collected and collated for the present study, the distribution of shoulder types at Indiana's rural two-lane state highways was investigated, for both functional class (Figure 6-22) and region (Figure 6-23). The type of shoulder for the road section is defined by four dummy variables as follows

$$
\begin{aligned}
& \text { 0-no shoulder, } \\
& \text { - earth, } \\
& \text { 2-stabilized, } \\
& 3 \text { - paved shoulders. }
\end{aligned}
$$

The bar charts are presented in order of increasing levels of "desirability" or quality. For example, it is least desirable to have no shoulder and most desirable to have a paved shoulder. The bar charts showed that the predominant shoulder type for rural two-lane collectors and minor arterials is the Stabilized type, while paved shoulders are most common at rural principal arterials. Also, almost $15 \%$ of sections on rural collectors have either no shoulder or earth shoulder while only 2-3 \% of rural minor and principal arterial sections fall in that category of shoulder type. This shows that from shoulder type considerations, there is a clearly decreasing shoulder quality from rural principal arterials to rural collectors.

From the perspective of regional variations in shoulder type (Figure 6-23), it is seen that northern road sections are slightly of less quality compared to central sections, but are of considerably higher quality than their southern counterparts. 

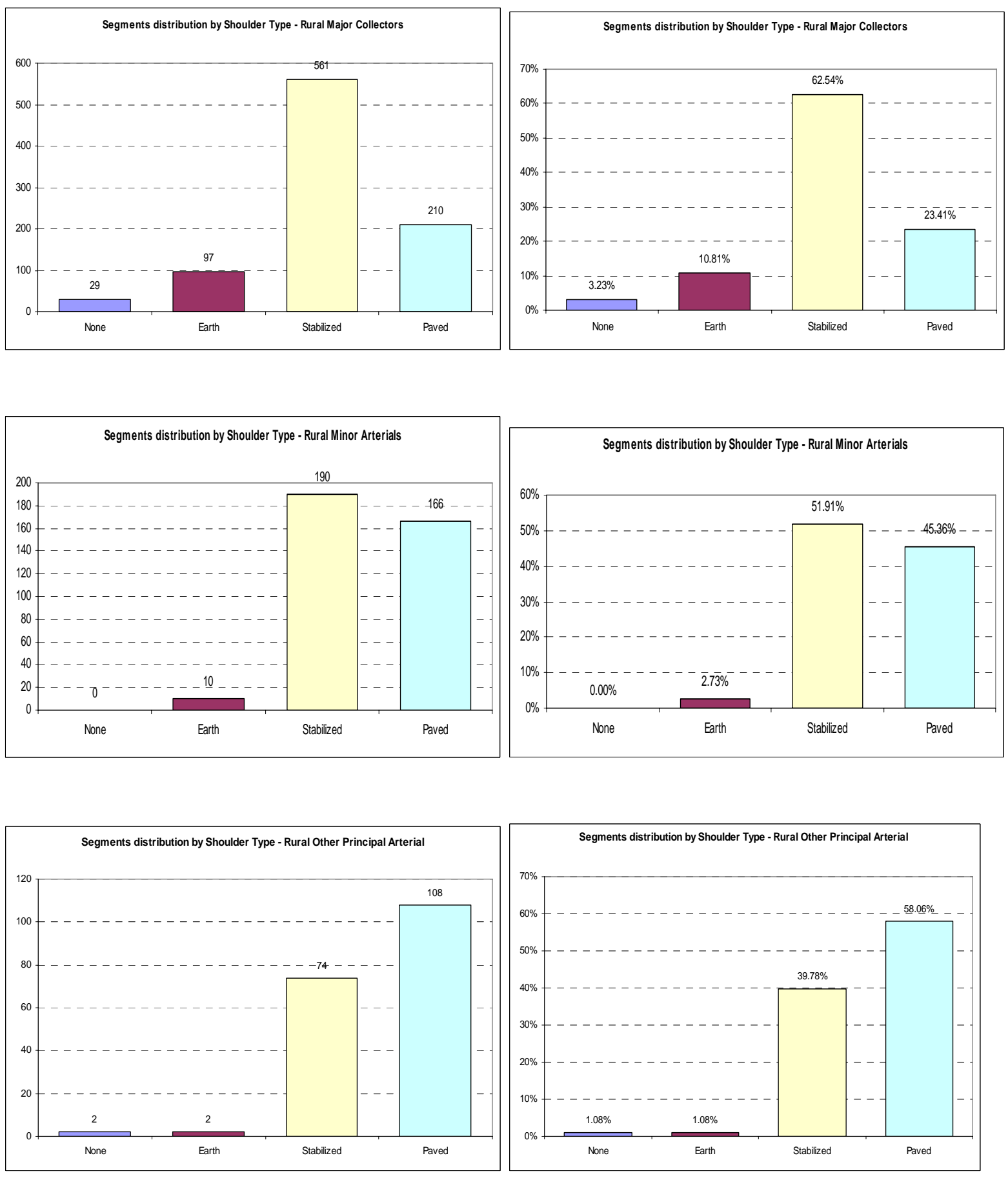

Figure 6-22: Distribution of Shoulder Type on Rural Two-lane Highways in Indiana, by Functional Class 

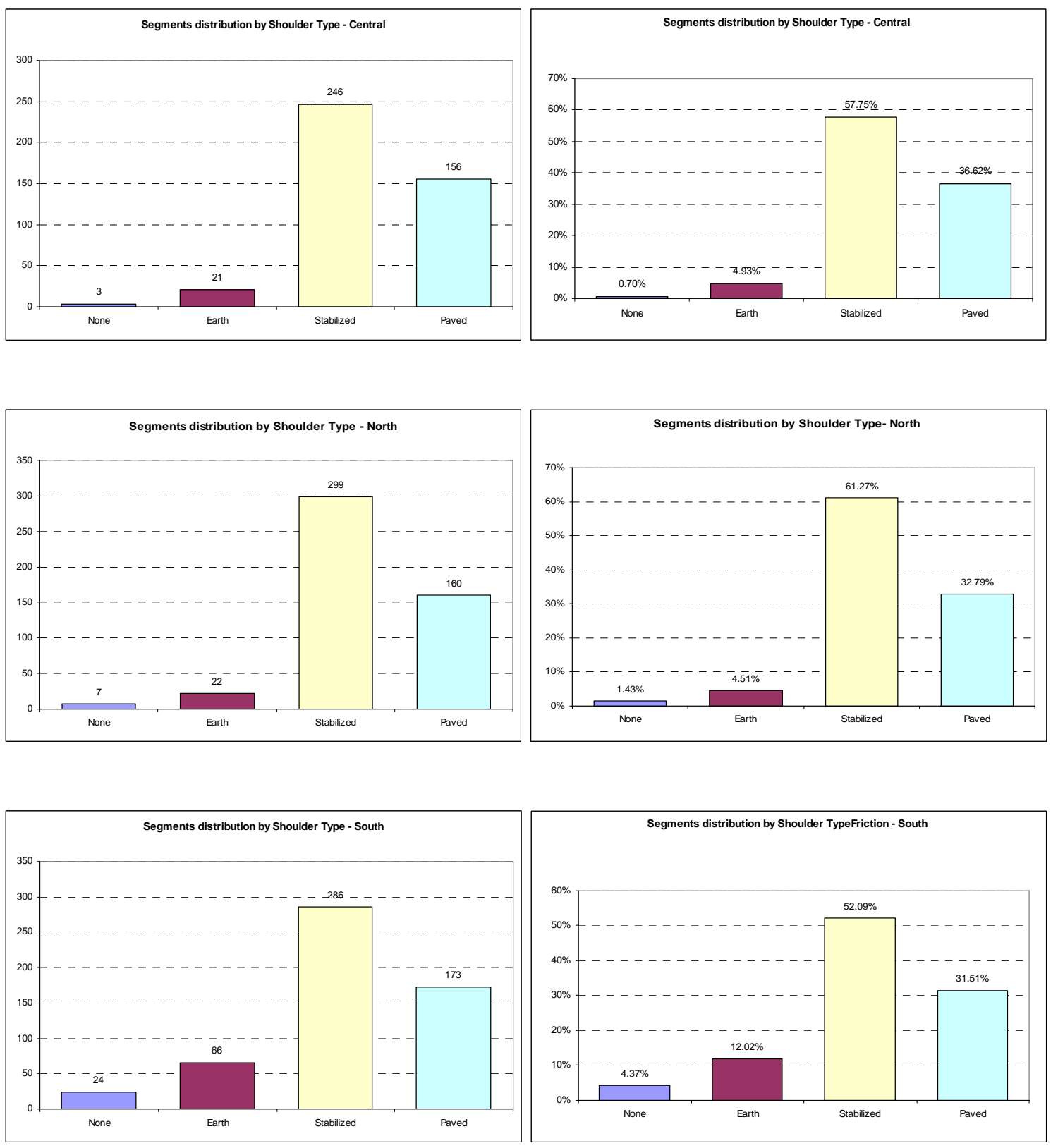

Figure 6-23: Distribution of Shoulder Type on Rural Two-lane Highways in Indiana, by Region 


\subsubsection{Variation of Crash Rates with Shoulder Type by Functional Class}

A preliminary descriptive analysis of the variation of crash rates with shoulder type was carried out. The ordinate axis shows the average annual crash rate per vehicle-milesof-travel (Figure 6-24).

As expected, the data showed that shoulder type is generally negatively correlated with crash rates, i.e., paved shoulders generally tends to be safer than non-paved shoulders, a result that is consistent with past research [Turner et al., 1981; Ogden, 1997].

Also, as one moves from an earth shoulder to a paved shoulder, higher surface friction that made available by the higher pavement surface quality helps bring a vehicle to a stop or from running off the road. As such, a lower crash rate is expected for paved shoulders compared to earth shoulders.
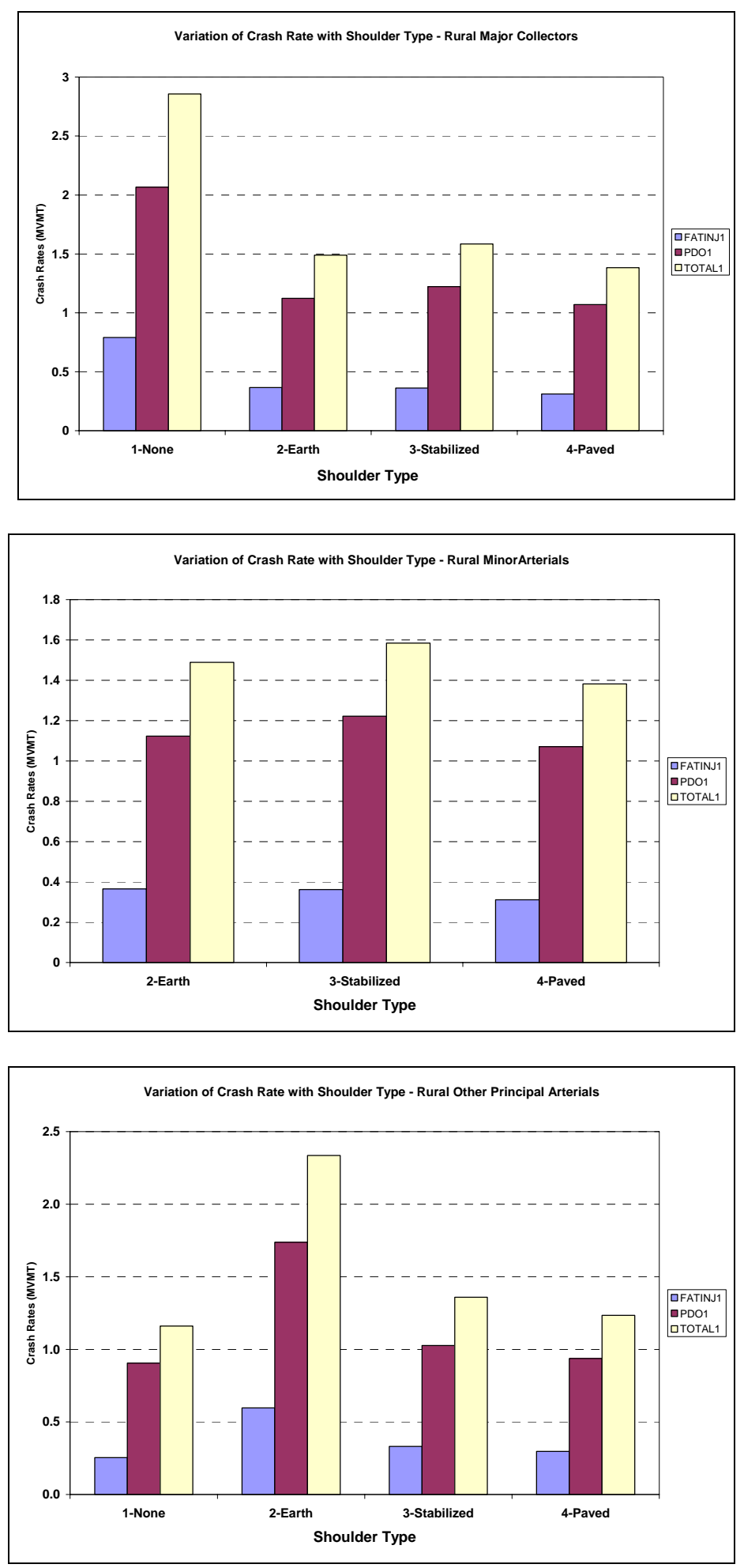

Figure 6-24: Variation of Crash Rates with Shoulder Type by Crash Severity and Functional Class 
From the Indiana data used in the present study, the correlation coefficients between shoulder width and crash rates are presented in Table 6-18 below. This is done for each crash severity category. The coefficients support the findings from the descriptive analysis: greater shoulder widths are associated with reduced crash rates (crashes per VMT).

Table 6-18: Correlation between Shoulder Type (Quality) and Crashes

\begin{tabular}{|c|c|c|c|c|c|}
\hline & $\begin{array}{c}\text { Fatal Crash } \\
\text { Rate }\end{array}$ & $\begin{array}{c}\text { Injury Crash } \\
\text { Rate }\end{array}$ & $\begin{array}{c}\text { PDO } \\
\text { Crash Rate }\end{array}$ & $\begin{array}{c}\text { Fatal or Injury } \\
\text { Crash Rate }\end{array}$ & $\begin{array}{c}\text { Total } \\
\text { Crash Rate }\end{array}$ \\
\hline $\begin{array}{c}\text { Shoulder Types at Rural Two- } \\
\text { Lane State Highways in Indiana }\end{array}$ & -0.0852 & -0.2411 & -0.1912 & -0.2442 & -0.2210 \\
\hline
\end{tabular}

\subsubsection{Shoulder Rumble Strips}

Shoulder rumble strips are raised or grooved patterns impressed on the paved shoulder of a highway, and are typically used to provide drivers with an audible and tactile warning that their vehicle is crossing the shoulder and that they are in danger of running off the road. Shoulder rumble strips may be milled, rolled, formed, or raised. Most states use milled rumble strips as such technique is easy to implement on new or old asphaltic or Portland cement concrete pavement. It has been shown in past research that milled rumble strips pose little or no threat to the structural or functional integrity of the shoulder, and they produce noise much louder than the other types of rumble strips. This section focuses on rumble strip applications on the carriageway, where such facility is provided on the centerline (Plate 6-5). At the present time, there was no information regarding the effectiveness of centerline rumble strips, but is it obvious that such countermeasure has great potential in reducing crashes on rural two lane highways in Indiana and elsewhere.

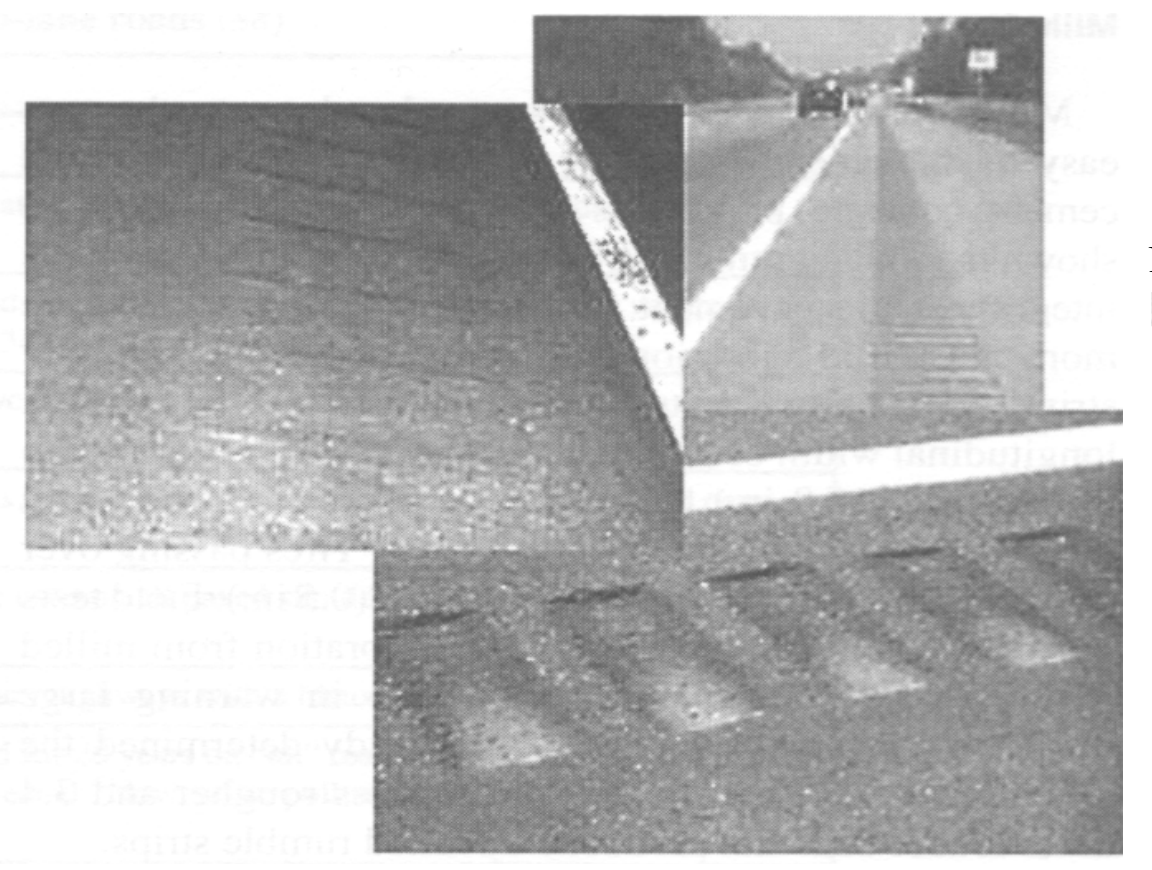

Plate 6-5: Shoulder Rumble Strips [Harwood et al., 2000] 
Several studies in the past have attested to the efficacy of rumble strips in reducing run-offroad crashes. It has been shown that rumble strips reduce the rate of run-off-road crashes by $15-70 \%$. Using data from New Jersey, it was shown in an FHWA study that installation of shoulder rumble strips yielded a 34\% reduction in run-off-road crashes even when overall crash rates increased by over 11\% [FHWA, 1999]. Hickey [1997] also reported that the Pennsylvania turnpike experienced a decrease in run-off-road crashes on their multi-lane facilities and a reduction of approximately 100 crashes per year was attributed to the use of shoulder rumble strips. Chaudoin and Nelson [1985] investigated the impacts of continuous rumble strips at seven rural highways in California, stating that the $49 \%$ reduction in crashes in the year following installation was attributed to the installation of the rumble strips. Also, in a FHWA study, Ligion et al. 1987] carried out a detailed analysis of sites with rumble strips, and found that such facilities led to a $20 \%$ decrease of run-off-road crashes, while the crash rates at comparable control sites increased by $9 \%$. Garder [1995] examined the effect of rumble strips on pedestrian crashes and also carried out a survey of 50 states regarding the use of rumble strips on two-lane roads. The results of the survey (Table 6-19) showed that the use of continuous rumble strips for roads other than limited access highways is fairly limited.

Table: 6-19: Rumble Strips - State of Practice

\begin{tabular}{|c|c|}
\hline STATE & POLICY \\
\hline 35 states & No practice \\
\hline Alabama & $\begin{array}{l}\text { Use continuous rumble strips to separate lanes for car traffic from shoulders designated for } \\
\text { bicyclist and pedestrian use. }\end{array}$ \\
\hline Arizona & $\begin{array}{l}\text { Treat all shoulders of rural divided and undivided roadways on which pavement width, } \\
\text { including shoulders, exceeds } 10.4 \mathrm{~m}(34 \mathrm{ft}) \text {. }\end{array}$ \\
\hline California & $\begin{array}{l}\text { Rumble strips are not used where bicyclists use the shoulder unless there is a } 1.5-\mathrm{m}(5-\mathrm{ft}) \\
\text { clear shoulder left on the outer edge. }\end{array}$ \\
\hline Colorado & $\begin{array}{l}\text { Roll strips into all bituminous overlays, as well as in new construction of all Portland cement } \\
\text { concrete highways. }\end{array}$ \\
\hline Georgia & $\begin{array}{l}\text { Continuous shoulder rumble strips are used on all paved shoulders that are at least } 1.2 \mathrm{~m} \\
(4 \mathrm{ft}) \text { wide. }\end{array}$ \\
\hline Idaho & Rumble strips are considered on primary highways with a history of run-off -road accidents. \\
\hline $\begin{array}{l}\text { Illinois } \\
\text { (Cooks County) }\end{array}$ & $\begin{array}{l}\text { Shoulder rumble strips have been used for } 20 \text { years on "all" resurfacing projects, and more } \\
\text { than a third of the network has been treated. Now, noise pollution and some opposition from } \\
\text { bicyclists have slowed new treatment. }\end{array}$ \\
\hline Kansas & Two-lane rural roads are treated if shoulders are wider than $1.8 \mathrm{~m}(6 \mathrm{ft})$. \\
\hline Kentucky & $\begin{array}{l}\text { Since } 1988 \text { shoulder rumble strips have been added to resurfacing, rehabilitation, and new } \\
\text { construction on all roads with wide, paved shoulders and narrow shoulders if monolithic. }\end{array}$ \\
\hline Missouri & $\begin{array}{l}\text { All roads with Portland cement concrete shoulders or bituminous lift at least } 45 \mathrm{~mm} \text { (1.75 in) } \\
\text { thick and at least } 1.2 \mathrm{~m} \text { ( } 4 \mathrm{ft} \text { ) wide get continuous shoulder rumble strips as long as the } \\
\text { shoulder is not expected to become a travel lane. }\end{array}$ \\
\hline Nevada & $\begin{array}{l}\text { All rehabilitation and overlay projects require rumble strips if the shoulder is } 1.2 \mathrm{~m}(4 \mathrm{ft}) \text { or } \\
\text { wider. }\end{array}$ \\
\hline New Mexico & $\begin{array}{l}\text { All rural highways get rumble strips when they are improved, except for smaller projects, } \\
\text { projects in mountainous terrain with many curves, or if shoulders are less than } 2.4 \mathrm{~m}(8 \mathrm{ft}) \\
\text { wide and used by many bicyclists. }\end{array}$ \\
\hline Pennsylvania & $\begin{array}{l}\text { Shoulders are treated if there are many run-off-road accidents and the shoulder is at least } 2.4 \\
\mathrm{~m}(8 \mathrm{ft}) \text { wide. }\end{array}$ \\
\hline Utah & $\begin{array}{l}\text { All two-lane two-way roads with safety problems or design speed more than } 50 \mathrm{mph} \text { and at } \\
\text { least } 1.2-\mathrm{m} \text { (4-ft) shoulders get rumble strips during reconstruction. }\end{array}$ \\
\hline West Virginia & $\begin{array}{l}\text { All U.S. and state routes with bituminous pavement get rumble strips if shoulders are at least } \\
2.4 \mathrm{~m} \text { ( } 8 \mathrm{ft} \text { ) wide (or adjacent to ramps and climbing lanes that have shoulders at least } 0.9 \mathrm{~m} \\
{[3 \mathrm{ft}] \text { wide). }}\end{array}$ \\
\hline
\end{tabular}




\subsection{Roadside Environment}

It has well been established in past research that the condition of the roadside affects the frequency and severity of crashes, and even influences the pattern of crashes. The roadside particularly affects run-off-road crashes which are known to be predominant on rural two-lane roads. It is expected that providing an environment that is more "tolerant" of driver errors and arrant vehicles will lead to reduction in such crashes. According to Fitzpatrick et al. [2000,] the relative hazard of the roadside may be described in terms of several features, such as:

- roadside recovery distance (or roadside clear zone)

- side-slopes (fore-slopes and back-slopes)

- presence of specific roadside obstacles (trees, culverts, utility poles, guardrails).

The impacts of each feature are discussed below.

\subsubsection{Recovery Distance}

The concept of a forgiving roadside recognizes that drivers may run their vehicles off the roadway and that serious crashes could be avoided or lessened if a traversable recovery area were made available. Such area should ideally be free of any obstacles such as trees, steep slopes, and utility poles. Fitzpatrick et al provide the following order by which treatment of obstructions could be carried out:

- Remove the obstacle or redesign it so that it can be traversed safely

- Relocate the obstacle to a point where it is less likely to be struck

- Reduce the impact severity by using an appropriate breakaway device

- Redirect a vehicle by shielding the obstacle with a longitudinal traffic barrier and/or crash cushion if it cannot be eliminated, relocated, or redesigned

- Delineate the obstacle if the above alternatives are not appropriate.

Conceptually, the roadside recovery distance is a relatively flat, unobstructed area adjacent to the travel lane where there is reasonable chance for an off-road vehicle to safely recover [Zegeer et al., 1987]. As such, it is measured as the distance from the outside edge of the travel lane to the nearest obstacle posed to errant vehicles.

Graham and Harwood [1982] determined the effect of clear zone policy on single-vehicle crash rates (Figure 6-25). As shown in the figure, single vehicle crashes per mile per year are highest for roads with no clear zone policy. The authors of that study duly noted that clear zone policies of the sample sections were not always exactly indicative of actual field conditions. However, the results 
obtained by the study provide revealing and interesting insights into the effect of clear roadside environment zones on safety.

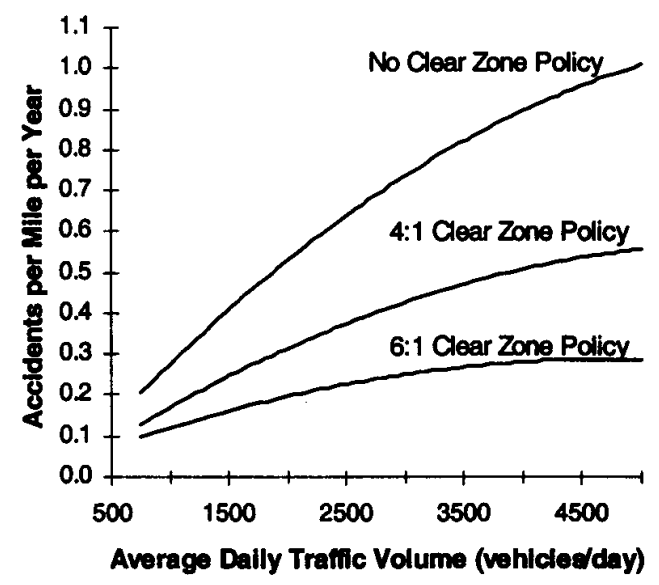

Figure: 6-25: Effect of Clear Zones on Safety [Graham and Harwood, 1982]

At areas where there is lack of a clear recovery zone, appropriate maintenance could be carried out to clear the obstacles and continue to maintain it that way. Fitzpatrick et al provide recommended roadside recovery distances (or clear zones). Examples of roadside improvements that could increase the recovery distance and therefore enhance the safety include cutting the trees next to the roadway, relocating utility poles further from the road, and the use of slide-slopes of 1:4 or flatter slopes. For roadways with limited recovery distances (particularly less than $15 \mathrm{ft}$ ) from the edge-line, the accident reductions corresponding to increases in recovery distance may be found from Table 620 [Zegeer et al., 1987]. For example, increasing the roadside recovery distance by $15 \mathrm{ft}$ is associated with a reduction in "related" crashes by 35\%. "Related" includes run-off-road, head-on, and sideswipe crashes.

Table 6-20: Accident Reduction Factors due to Increasing Roadside Recovery Distance

\begin{tabular}{|c|c|}
\hline $\begin{array}{c}\text { Amount of Increased Roadside } \\
\text { Recovery Distance (ft) }\end{array}$ & $\begin{array}{c}\% \text { Reduction in "Related" } \\
\text { Crash Types }\end{array}$ \\
\hline 5 & 13 \\
\hline 8 & 21 \\
\hline 10 & 25 \\
\hline 12 & 29 \\
\hline 15 & 35 \\
\hline 20 & 44 \\
\hline
\end{tabular}

Note: These values are only for rural two-lane roads, Source: [Zegeer et al., 1987] 


\subsubsection{Side Slopes}

The steepness of the roadside slopes is a cross-sectional feature that influences the ability of an errant vehicle to recover or the likelihood of an off-road vehicle to roll over. According to Fitzpatrick et al. [2000], existing guidelines for acceptable slopes have historically been based on computer simulations and observations of controlled vehicle test runs on various slopes, as well as informed judgments. In their 1997 FHWA study, Zegeer et al. [1997] investigated the relationship between the relative frequency of single vehicle crashes and field-measured side-slopes while duly controlling for other factors (Figure 6-26). It is seen that the relative frequency of single vehicle crashes is highest when the slope is steepest.

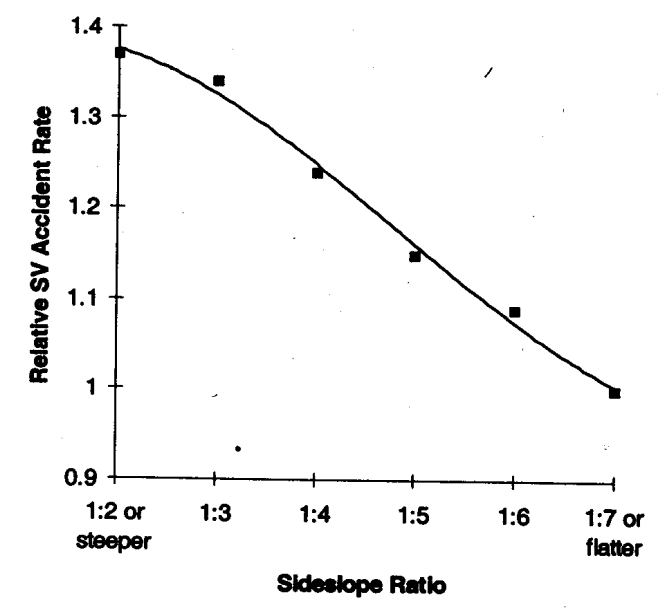

Figure 6-26: Relationship between Side-slopes and Single-vehicle Crash Rate

The needed countermeasure to address a problem of inadequate side slopes depends on the nature of the terrain relative to the roadway, i.e., whether the roadway is a fill section, a cut section or whether it is on level terrain with side ditches.

Fill Sections: At fill sections, side-slopes that are 1:4 or flatter are generally considered desirable, as drivers encroaching on the side-slope are afforded the opportunity to stop their vehicles or reduce speed enough to allow for safe recovery (as long as the recovery zone is free of obstacles). When the side-slopes are slightly steeper (between 1:3 and 1:4), it is possible to traverse such slopes, but most drivers would face much difficulty in stopping or returning the vehicle to the roadway. At such sections, a run-out area (as illustrated in Figure 6-27) may be required at the toe of the non-recoverable slope of the recovery area. Side slopes that are greater than 1:3 are considered to be critical because a vehicle that leaves the roadway is likely to overturn on such slopes [Fitzpatrick et al., 2000]. If a side 
slope steeper than 1:3 begins closer to the traveled way than the suggested clear zone distance, a barrier might be warranted if flattening the slope is not feasible.

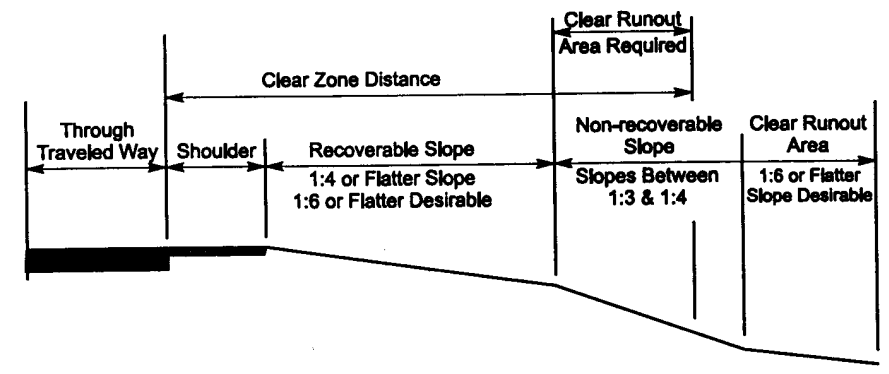

Figure 6-27: Illustration of Side-slope Design at Fill Section

Cut Sections: If the road is at a cut section, the back-slope may be traversable depending on its relative smoothness, slope, and presence of fixed obstacles. On one extreme, a 1:3 or flatter slope that is smooth and free of obstacles is easily to traverse and may serve a good recovery zone. On the other extreme, a steep, rough-surfaced rock cut should begin outside the clear zone or could be shielded from errant vehicles using an appropriate roadside barrier.

Ditch Sections: Ditches often constitute a unique roadside hazard. Ditches are designed primarily to collect and convey storm water runoff, however, for safety purposes, it is imperative that their design gives due consideration to safety contingencies such as helping an errant vehicle to recover with minimal injury to its occupants. AASHTO’s Roadside Design Guide [AASHTO, 1996] provides guidelines of fore-slopes and back-slopes for basic ditch configurations, and provides figures that guide the designer on selecting slopes that may be traversed by errant vehicles. Fitzpatrick et al. [2000] suggested the following improvements to ditches located in vulnerable area and whose cross sections need improvements:

- The ditch could be reshaped to conform to a more forgiving design

- The ditch could be converted to a closed drainage system using culverts and pipes

- The ditch could be shielded from traffic using a traffic barrier.

The relationship shown in Figure 6-26 was used to develop crash reductions matching various side-slope flattening projects. The percent reductions are presented in Table 6-21 for single vehicle and total crashes. For example flattening an existing side-slope to 1:6 should result in a reduction of approximately $21 \%$ and $12 \%$ of single-vehicle and total crashes, respectively [Zegeer et al., 1987]. For such reductions, it is assumed that the roadside slope to be flattened is relatively clear of obstacles. 
Using flatter slopes not only reduces the crash rates, but may also reduce the severity of rollover crashes which are typically associated with fatalities or/and serious injuries. Injury data from three states indicate that 55\% of run-off-road rollover crashes result in occupant injury, and up to 3\% result in death. The Zegeer et al. study showed that side-slopes of 1:5 or flatter are needed to significantly reduce the incidence of rollover crashes.

Table 6-21: Accident Reduction Factors due to Increasing Roadside Recovery Distance

\begin{tabular}{|c|c|c|c|c|c|c|c|c|}
\hline \multirow{2}{*}{$\begin{array}{c}\text { Side-slope before } \\
\text { Flattening }\end{array}$} & \multicolumn{9}{|c|}{ Side-slope after flattening } & \multicolumn{2}{c|}{$1: 6$} & \multicolumn{2}{c|}{$1: 5$} & \multicolumn{2}{c|}{ or Flatter } \\
\cline { 2 - 10 } & $\begin{array}{c}\text { Single } \\
\text { Vehicle }\end{array}$ & Total & $\begin{array}{c}\text { Single } \\
\text { Vehicle }\end{array}$ & Total & $\begin{array}{c}\text { Single } \\
\text { Vehicle }\end{array}$ & Total & $\begin{array}{c}\text { Single } \\
\text { Vehicle }\end{array}$ & \begin{tabular}{c} 
Total \\
\hline $1: 2$
\end{tabular} \\
\cline { 2 - 10 }$y$ & 8 & 5 & 15 & 9 & 21 & 12 & 27 & 15 \\
\hline $1: 3$ & 0 & - & 6 & 3 & 12 & 7 & 19 & 11 \\
\hline $1: 4$ & - & - & 0 & - & 6 & 3 & 14 & 8 \\
\hline $1: 5$ & - & - & - & - & 0 & - & 8 & 5 \\
\hline
\end{tabular}

Note: These values are only for rural two-lane roads.

Source: [Zegeer et al., 1987]

\subsubsection{Obstacles}

Any natural or man-made obstacle located on the roadside has the potential for being hazardous. As such, it is important to remove such obstacles, protect the road user from them, or if they are unremovable, to render such obstacles as "forgiving" as possible in case a vehicle runs off the road. In the seventies, a study was carried out for the FHWA by Perchonok et al. [1978] to analyze the characteristics of single-vehicle crashes, including the severity of crashes related to the type of "obstacle" struck. It was found that for non-rollover fixed-object crashes, the obstacles associated with the highest percentage of injury occurrences are as follows:

- bridge and overpass entrances (75\% injured, and $15.9 \%$ fatality)

- trees (68\% injured, and $7.2 \%$ fatality)

- field approaches, i.e., ditches created by driveways (67\% injured, and $1.3 \%$ fatality)

- culverts (62.3\% injured, and $6.1 \%$ fatality)

- embankments (57.6\% injured, and $4.4 \%$ fatality)

- wooden utility poles (51.2\% injured, and $2.3 \%$ fatality)

Of the obstacle types studied, those with relatively fewest injury and fatality rates were rocks, ditch, fences, and signposts. 
Countermeasures to address the problem of obstacles are categorized as follows: remove the obstacle and construct a barrier to protect the road user from the obstacle. The most common obstacles that are found on Indiana's rural two-lane system are roadside signs, drainage structure, trees, mailbox supports, and utility poles. At county roads, the most common obstacles are fence walls and trees.

\subsubsection{Roadside signs:}

These can be divided into three categories: overhead signs, large roadside signs, and small roadside signs. Overhead signs typically require massive support systems that cannot be made to yield upon being struck by an errant vehicle. As such, they should be installed on nearby overpasses or other structures where possible. If it is not possible to locate the supports of overhead signs outside the recovery zone, Fitzpatrick et al. [2000] suggested that such supports could be shielded with an appropriate barrier. Large roadsigns (typically exceeding $53.6 \mathrm{ft}^{2}$ in area and supported by two or more posts), resist wind and ice loads, but are designed to breakaway upon being struck by the vehicle. Small roadsigns (typically not exceeding $53.6 \mathrm{ft} 2$ in area and supported by one or two posts), are also designed to resists wind and ice loads, but to breakaway upon being struck by the vehicle. AASHTO’s Roadside Design Guide offers guidelines on how to design breakaway supports for roadside signs. As such, any future road safety audit of rural two-lane roads in Indiana could make use of such guidelines to determine whether existing roadsigns on that network are consistent with specification.

Addressing the problem of roadside signs is a two-edged sword: signs should be far enough from the road so that they (or their supports) do not pose an obstacle and safety hazard, but should be close enough to be legible. It is recommended in literature that the sign placement must be within the drivers' cone of vision, and that signs could be designed in such a way as to collapse upon impact by errant vehicles. Table 6-22 [Zegeer et al., 1981] presents the expected reduction in sign related crashes if signs are moved further away from the roadway.

An analysis of the impacts of absence of roadsigns at critical areas could not be carried out due to lack of data on the inventory of such signs at the rural two-lane sections. As such, the effectiveness of this countermeasure could not be investigated with Indiana data in the present study. A central pillar of Indiana's Asset Management System is the collection of data on all the state's assets including road signs. After implementation of such a system, requisite data would be available for impact analysis of roadsigns to be carried out. 


\subsubsection{Drainage Structures:}

Drainage features on the state's rural two-lane system were generally designed and built with considerations of hydraulic efficiency as well as roadside safety. Common drainage structures that may constitute a safety hazard to errant vehicles include culvert headwalls. This problem is typically encountered when the culvert is too narrow and may need widening.

Culvert headwalls can result in serious injury or death when struck at moderate or high speeds on rural roadways. It may be feasible, under certain conditions, to relocate such culverts further from the roadway, but the ideal solution would be to reconstruct the drainage facilities so that they are flush with the roadside terrain so that they pose no obstacle to vehicles. Fitzpatrick et al. [2000] sugggest that such designs would virtually eliminate culvert-related crashes, although some run-off-road vehicles denied such restraint by the culvert headwall could go on either to strike other obstacles (such as trees) that are located beyond the culverts or rollover on a steep side-slope. Table 6-22 [Zegeer et al., 1990] presents the potential reductions in crashes in response to placement of culvert headwalls further away from the roadway. The table shows that extending the culvert headwalls by $5 \mathrm{ft}$ for example, could yield a 23\% reduction in related crashes. AASHTO's Roadway Design Guide offers some interesting safety design features of culvert headwalls.

\subsubsection{Trees:}

Trees can constitute potential obstructions by virtue of the size and location in relation to passing traffic [Fitzpatrick et al., 2000]. A single tree with a trunk diameter exceeding 5.9 inches is considered a "fixed object." Also, when trees or shrubs with multiple trunks or groups of small trees are together, they may be considered as having the effect of a single tree with their combined cross sectional area. Addressing the tree hazard is generally carried out in one of two ways: on-roadway treatments (such as pavement markings, signs, and roadway improvements that are designed to help the driver stay on the roadway), and off-roadway treatments (such as tree removal and shielding that are meant to remove the obstacle or protect the driver from it). It is often recommended to consider tree removal particularly when trees are determined to be obstructions and are also located at location where they constitute a likely hazard.

The expected reduction in crashes due to tree removal can be estimated using the crash reduction percentages shown in Table 6-22 below. The table shows that clearing all trees within an additional $10 \mathrm{ft}$ yields a reduction in tree crashes by $57 \%$. The use of these values is inherent with the assumption that clearing trees back from the roadway makes available increased recovery space for run-off-road vehicles provided the trees were not on a steep side-slope. According to Zegeer et al. 
[1990], trees are the fixed object most often struck on many rural roads, and therefore clearing trees back from the road (particularly on roads with sever alignment) has tremendous potential in reducing crashes that occur at such highways.

Table 6-22: Effectiveness of Treatments for Various Obstacles (Source: Zegeer et al. [1990])

\begin{tabular}{|c|c|c|c|c|}
\hline \multirow[b]{2}{*}{$\begin{array}{c}\text { Increase in Distance between } \\
\text { Obstacle and Roadway (ft) }\end{array}$} & \multicolumn{4}{|c|}{$\%$ Reduction in Crashes } \\
\hline & Trees & $\begin{array}{c}\text { Mailboxes, } \\
\text { Culverts \& Signs }\end{array}$ & Guardrails & Fences/Gates \\
\hline 3 & 22 & 14 & 36 & 20 \\
\hline 5 & 34 & 23 & 53 & 30 \\
\hline 8 & 49 & 34 & 70 & 44 \\
\hline 10 & 57 & 40 & 78 & 52 \\
\hline 13 & 66 & N.F. & N.F. & N.F. \\
\hline 16 & 71 & N.F. & N.F. & N.F. \\
\hline
\end{tabular}

An analysis of the impacts of roadside trees using Indiana data could not be carried out due to lack of data on the inventory of trees at Indiana's rural two-lane sections. As such, the effectiveness of "tree-removal" countermeasures could not be investigated with Indiana data for the present study.

\subsubsection{Mailboxes:}

Mailboxes serve a useful communication purpose for the society but are associated with possible hazard to road users [Ross et al., 1980; Zegeer et al., 1990; Fitzpatrick et al., 2000] and are also a source of increasing tort liability against the state of Indiana due to the damage they suffer during snow removal operations [Giraud et al., 2003]. Ross et al. [1980] investigated the safety hazard posed by mailboxes on rural roads in Texas.

AASHTO's Guide for Erecting Mailboxes on Highways [AASHTO, 1984] provides guidelines on how to construct mailboxes at a safe location from the roadway. Relocating mailboxes further from the edge of the roadway is expected to reduce the frequency of mailbox related crashes, as shown in Table 6-22. However, due to the nature of mail delivery it may not be practical to relocate such mailboxes beyond a certain distance. Zegeer et al [1990] recommend that the severity (but not the frequency) of mailbox related crashes could be reduced by the use of less rigid posts or breakaway designs in place of heavy steel or wooden posts, or multiple posts. The efficacy of such breakaway designs in reducing mailbox related crashes was demonstrated by Ross et al. [1980]. 


\subsubsection{Guardrails:}

The paradoxical situation with guardrails is that they serve to prevent vehicles from running off the road, but could end up posing a serious obstacle to errant vehicles. Nevertheless, it is worth noting that guardrails shield a vehicle from encountering more serious dangers than they themselves pose. They protect errant vehicles from striking more rigid obstacles such as trees or from rolling over steep embankments. Guardrails are typically installed in such a manner to enhance their function while minimizing their potential threat as obstacles. This is done by locating them as far from the roadway as allowed by technical and cost considerations. Thus, relocating guardrails to a safer distance can yield reductions in guardrail related crashes, and the expected reductions can be estimated using the relationships developed in a 1990 study (shown in Table 6-22). Fitzpatrick et al. [2000] contend that in cases of guardrails protecting against rollovers at steep sections, the guardrail should be removed when it is feasible to flatten the slopes to a relatively mild slope (such as 1:5 or flatter), because the guardrail constitutes an obstacle which can be struck by errant vehicles.

In the present study, an analysis of the impacts of guardrails could not be carried out due to lack of data on the inventory of such features at Indiana's rural two-lane sections.

\subsubsection{Fences and Gates:}

Unlike the case for state highways, the width of acquired right-of-way for county roads is relatively small. In this context, many county roads in Indiana have a problem of fences and gates located very close to the roadway, posing a significant threat to passing vehicles that happen to run off the road. Furthermore, damage to such properties by road users could result in increased exposure of the state or local highway agency to tort suits by property owners. Using nationwide data, Zegeer et al [1990] investigated the relationship between crashes and the roadway-to-fence distance, and found that significant savings in crashes could be accrued by relocating the fence further from the roadway (Table 6-22). The table shows that $10 \mathrm{ft}$ of relocation further from the roadway could yield as much as $52 \%$ reduction in fence related crashes. The only drawback to fence relocation lies in the need for INDOT or the county highway agency to acquire the needed right-of-way which is typically expensive.

An analysis of the impacts of roadside fences could not be carried out due to lack of data on the inventory of such features at Indiana's rural two-lane sections. As such, for the present study, the effectiveness of "fence-removal" countermeasures could not be investigated with Indiana data. 


\subsubsection{Utility Poles}

Strictly speaking, utility poles fall under the category of obstacles, but are hereby separated for discussion because of the unique issues associated with such types of obstacles. Utility poles represent a significant hazard on rural two-lane roadways, and it has been estimated that motor vehicle collisions with utility poles result in approximately 10 percent of all fixed-object fatal crashes annually, in the United States. Fitzpatrick et al [2000] identified three factors are influential to the frequency and severity of crashes with utility poles:

- Pole density, defined as the number of utility poles per mile

- Pole offset, defined as the proximity of the poles to the edge of the carriageway

- Pole rigidity, defined as the ability of the pole to breakaway upon impact.

For existing utility poles, Fitzpatrick et al. [2000] present the following possible countermeasures:

- placing utility lines underground

- increasing the lateral pole offset

- increasing pole spacing (decreasing pole density)

- installing breakaway utility poles

- placing barriers around utility poles.

How effective are these countermeasures in reducing crashes? The sections below describe the findings of previous studies that investigated the effectiveness of each of the five treatments.

\subsubsection{Placing Utilities Underground:}

Theoretically, placing utility lines underground is expected to eliminate $100 \%$ of the run-off-road plus fixed object crashes (where errant vehicle runs off the road and strikes the first object it encounters). In cases where there are other obstacles besides the utility poles, relocation of the poles simply transfers the first obstacle "honor" to other objects in the roadway clear zone. In such cases, placing utilities underground may yield little reduction in such types of crashes. A 1984 study carried out for FHWA suggested that burying the utilities underground can result in a 38 percent reduction in fatal crashes [Zegeer et al., 1984].

\subsubsection{Pole Offset:}

Fitzpatrick et al. [2000] state that relocating utility poles further from the roadway will generally reduce the frequency of utility pole crashes. Table 6-23 shows the reduction on utility poles crash frequency as a result of increasing the offset of utility poles from the pavement edge. At the present 
time, there is no indication of any past research or literature that demonstrates that increasing the pole offset is associated with reduced severity of pole crashes.

Table 6-23: Reduction in Utility Pole Crashes due to Pole Relocation

\begin{tabular}{|c|c|c|c|c|c|c|c|c|c|c|c|}
\hline \multirow{2}{*}{$\begin{array}{c}\text { Pole Offset } \\
\text { Refore } \\
\text { Relocation (ft) }\end{array}$} & \multicolumn{8}{|c|}{ Expected \% Reduction in Utility Pole Crashes } \\
\cline { 2 - 14 } & \multicolumn{10}{|c|}{ Pole Offset after Relocation } \\
\cline { 2 - 15 } & 6 & 7 & 8 & 9 & 10 & 11 & 12 & 13 & 14 & 15 & $20-30$ \\
\hline 4 & 30 & 42 & 49 & 55 & 60 & 63 & 69 & 70 & 72 & 73 & 77 \\
\hline 5 & - & 36 & 43 & 50 & 56 & 59 & 65 & 67 & 69 & 70 & 74 \\
\hline 6 & - & - & 27 & 36 & 43 & 48 & 55 & 57 & 60 & 62 & 67 \\
\hline 7 & - & - & - & 22 & 31 & 37 & 46 & 48 & 52 & 54 & 59 \\
\hline 8 & - & - & - & - & 22 & 29 & 39 & 42 & 45 & 48 & 55 \\
\hline 9 & - & - & - & - & - & 18 & 30 & 33 & 37 & 40 & 48 \\
\hline 10 & - & - & - & - & - & - & 22 & 25 & 30 & 33 & 42 \\
\hline 11 & - & - & - & - & - & - & - & 18 & 24 & 27 & 36 \\
\hline 12 & - & - & - & - & - & - & - & - & 11 & 15 & 25 \\
\hline 13 & - & - & - & - & - & - & - & - & - & 11 & 22 \\
\hline 14 & - & - & - & - & - & - & - & - & - & - & 17 \\
\hline
\end{tabular}

Source: [Zegeer et al., 1984]

\subsubsection{Reducing Pole Density:}

In their 1984 FHWA study, Zegeer et al. examined the crash frequencies associated with pole offsets categorized using three levels of pole density: high, moderate, and low (Figure 6-28), and found that lower pole density reduces pole crashes, all else being equal. The chart can be used to estimate the reduction in utility pole crash frequency as a result of reducing the pole density in the right-of-way.

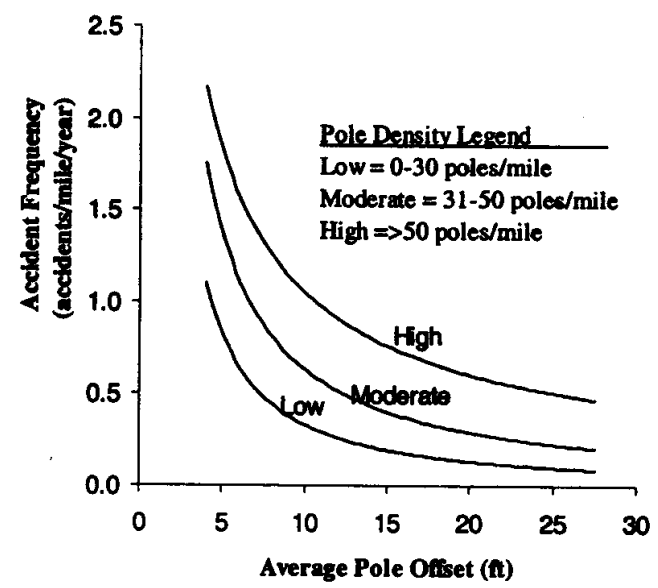

Figure 6-28: Relationship between Frequency of Utility Pole Crashes and Pole Offset/Density 
For simultaneous consideration of both pole offset and pole density, Figure 6-29 presents a nomograph developed to relate the frequency of vehicle crashes with utility pole density, as a function of traffic volume (ADT), average pole offset, and pole density. The nomograph can be used to estimate the expected effectiveness of increasing average pole offset or reducing pole density.

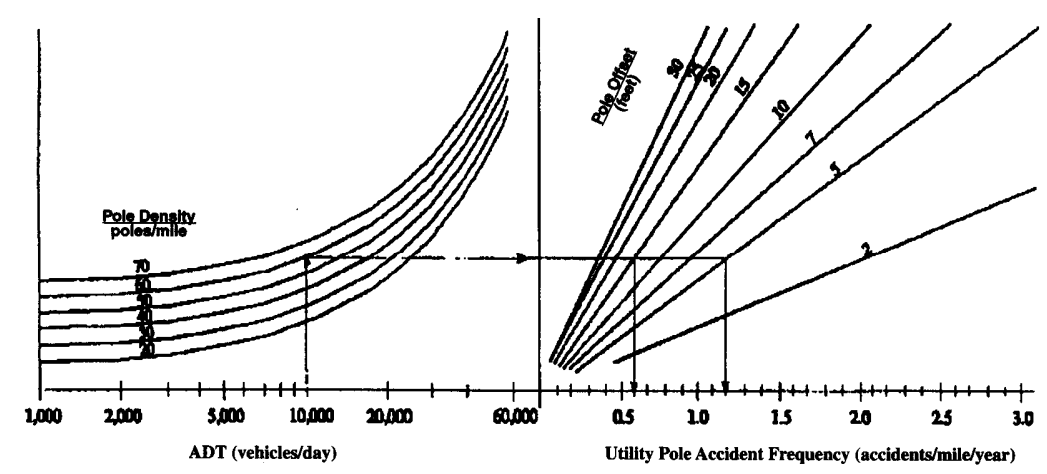

Figure 6-29: Nomograph for Predicting Utility Pole Crash Frequency [Zegeer et al., 1984]

Fitzpatrick et al. [2000] recommended that a site-specific benefit-cost analysis could be performed to decide on which of the five identified corrective measures is most cost-effective at a specific location with high crash frequency due to utility poles. Furthermore, the researchers duly recognized that most utility poles are owned and installed by private entities (even though these are installed on public right-of-way). As such, they are not under the direct control of the public agency, and this situation complicates the implementation of countermeasures geared at reducing the extent of the threat they pose to passing traffic.

In the present study, an analysis of the impacts of roadside utility poles could not be carried out due to lack of data on the inventory of utility pole locations at Indiana's rural two-lane sections.

\subsubsection{Roadside Hazard Ratings}

A collective index that embodies the effects of the factors discussed in the previous sections (side slopes, obstacles, utility poles, etc.) has been established in previous research and used in a number of safety studies. Zegeer et al. [1987] developed a 7-point roadside hazard scale based on a literature review and a survey of roadside safety professionals (Plate 6-6). This is a largely subjective measure of all hazards associated with the roadside environment. The rating values indicate the crash likelihood 
and damage severity expected to be sustained by errant vehicles on a scale of 1 (low likelihood of offroadway collision or overturn) to 7 (high likelihood of a crash resulting in a fatality or sever injury).

An analysis of the impacts of roadside hazard could not be carried out due to lack of data on the inventory of trees at Indiana's rural two-lane sections. There are indications that INDOT's Safety Management Unit is currently collecting data on roadside hazard ratings for the state highway system. After such field data has been collected, it would be possible to investigate the relationships between roadside hazard ratings and crash rates. Also, it would be possible to derive crash reduction factors that quantify the benefits or effectiveness of actions that improve roadside hazard ratings.

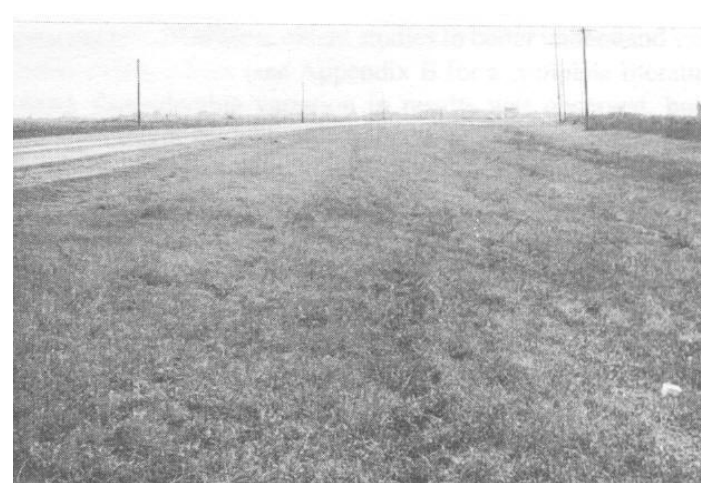

Roadside Hazard Rating of 1

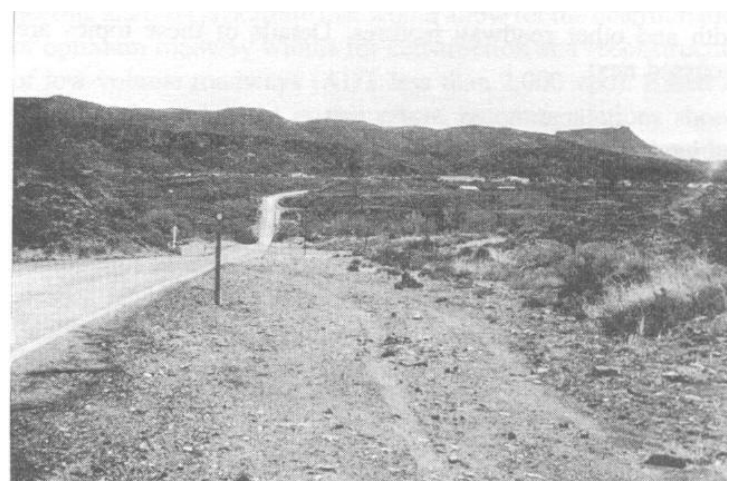

Roadside Hazard Rating of 3

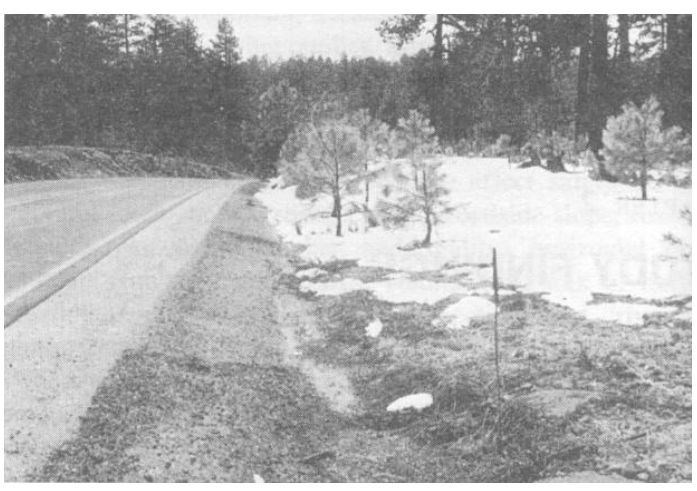

Roadside Hazard Rating of 2

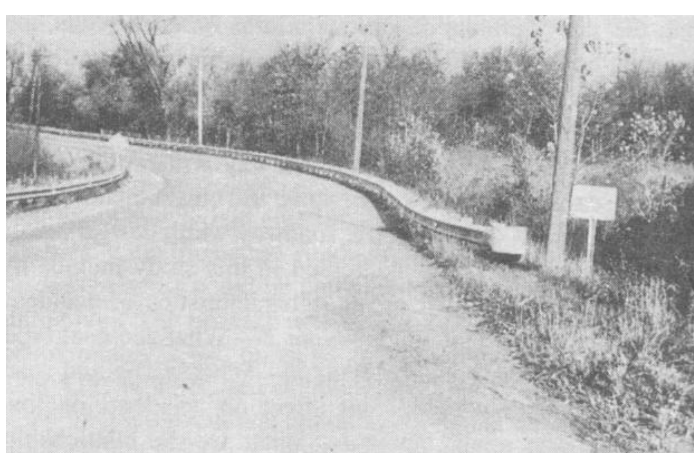

Roadside Hazard Rating of 4

Plate 6-6a: Road Side Hazard Ratings - Conceptual Illustrations 


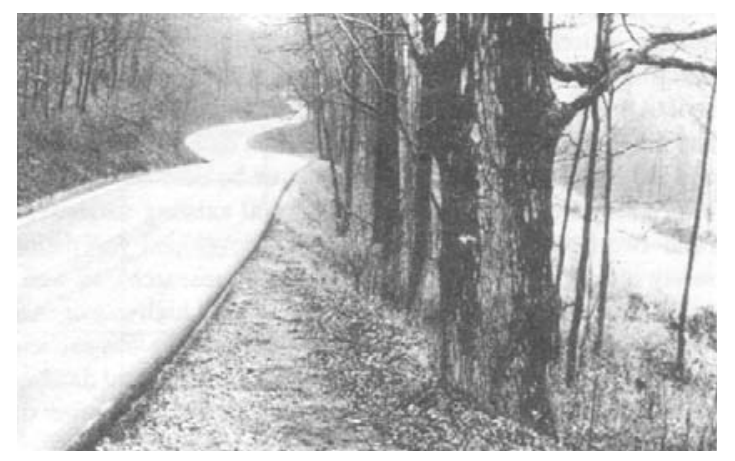

Roadside Hazard Rating of 5

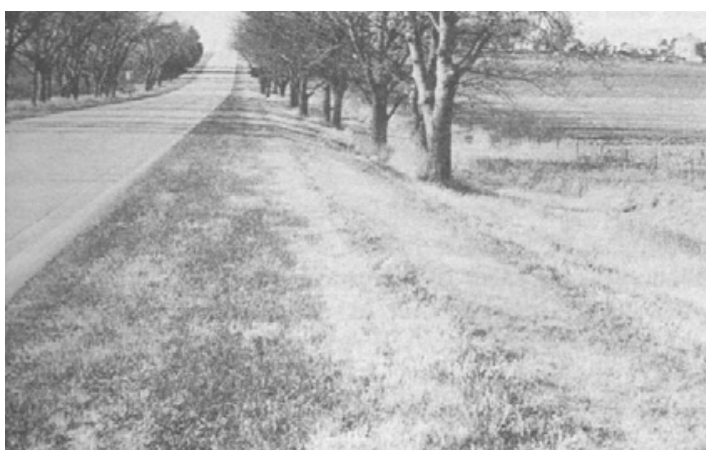

Roadside Hazard Rating of 6

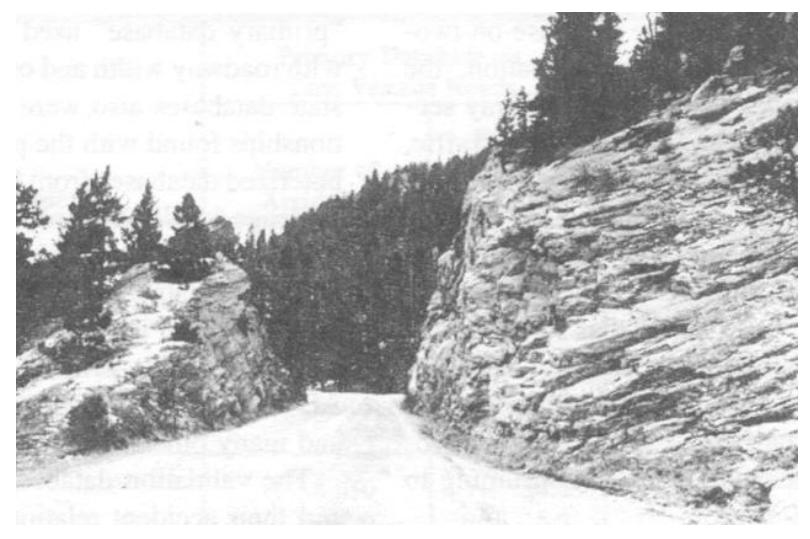

Roadside Hazard Rating of 7

Plate 6-6b: Road Side Hazard Ratings: Conceptual Illustrations

\subsubsection{Access Management}

Highway access management plays an important role in promoting highway safety by providing appropriate access to the highway while minimizing traffic conflicts. On one extreme, principal arterials have limited access and maximum mobility, while on the other extreme, local roads are associated with maximum access and limited mobility. According to Fitzpatrick et al. [2000], access management is typically thought of as an urban issue but can also be applied to rural areas to address problems of performance and safety. Tarko et al. [2000] carried out an access management study for highways in Indiana and identified key concepts for highway access management, such as restriction of turning movements, signalization, and encouragement of shared driveways, etc. Lalani [1998] 
indicated that a well-designed and effectively administered access management plan can reduce crash rates by $40-60 \%$.

Arterials are generally major multi-lane or intercity roads, usually with some controlled access. Collector roads are smaller scale roads that generally connect local distributor roads with arterials generally with little or no access control. Local and county roads have the least level of access control (Plate 6-7).

\subsubsection{Distribution of Access Management Features on Rural Two-lane Highways in Indiana, by Functional Class}

Due to lack of variation in the data relating to access control, the access control factor was not considered in the present study for state highways. However, it was given due consideration in the present study for county roads, where access control (or lack thereof) was measured in terms of driveway density.

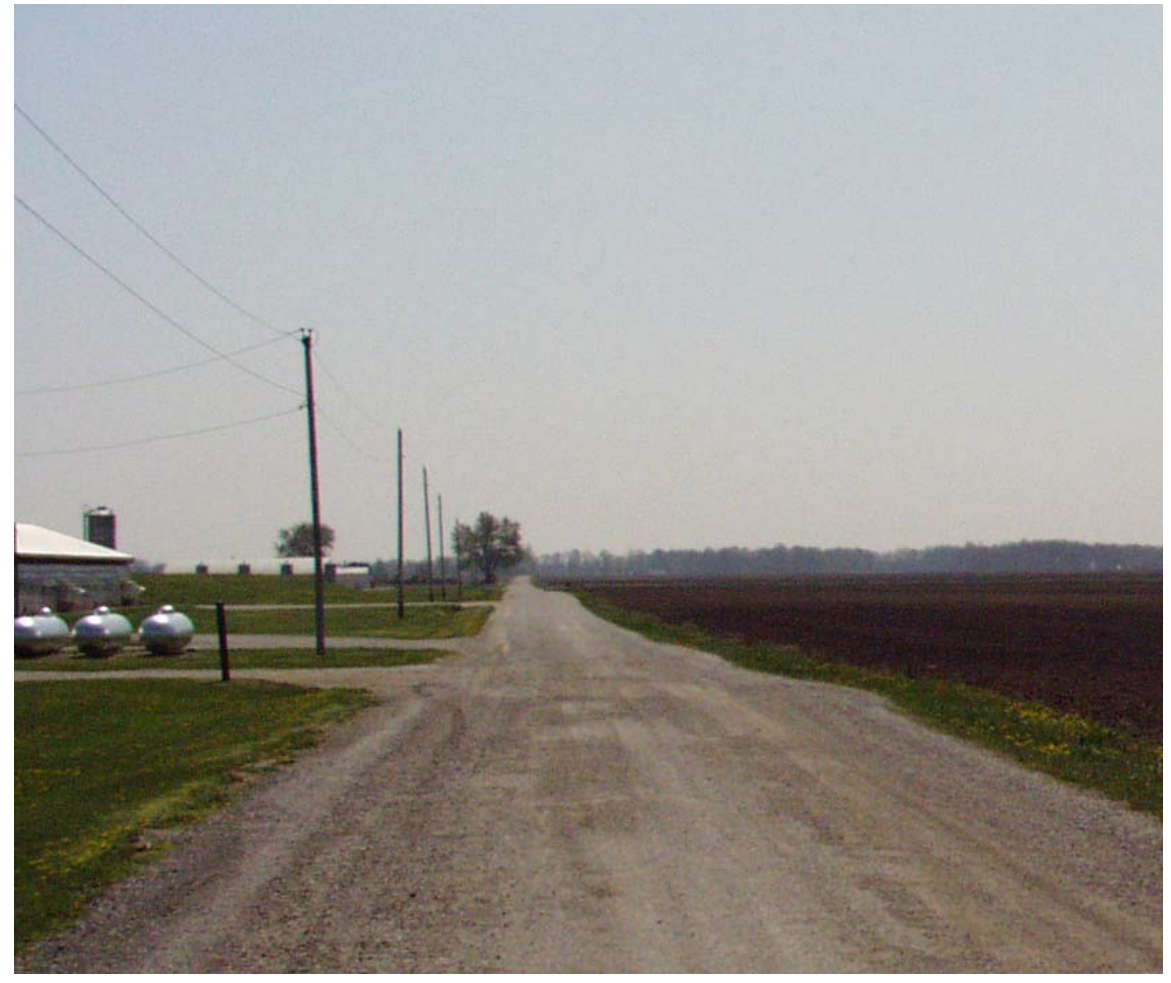

Plate 6-7: Higher Number of Driveways at County Road Sections are Associated with Higher Crashes 


\subsubsection{Crossing Animals}

In Indiana, crash occurrence between passing vehicles and large animals at rural road sections is a significant problem. There are indications that in 1998, INDOT commissioned a study to assess the extent of the problem, and to investigate the possible remedies to reduce the incidence of such crashes. In a nationwide study by Nelson [1994], it was shown that the major "culprit" is the white tailed deer (Plate 6-8, from Fitzpatrick [2000]) whose current fast-growing population of 25 million in the lower 48 states has doubled over the last decade. It has been found that deer are attracted to highways, partly because of salt that has leached into the soil surrounding the highway from the road surface (due to winter-time salting operations), and partly because of foliage planted in the median and along the roadway. Also, deer cross roadways to move from open feeding areas to protected bedding areas in regular cycles, sometimes several times a day [Fitzpatrick, 2000].

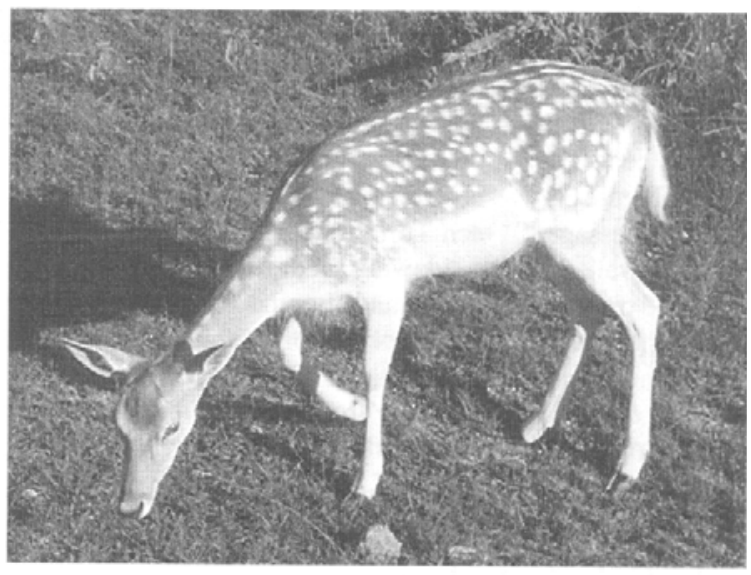

Plate 6-8: The White-tailed Deer is a Common Cause of Crashes at Indiana's Rural Two-Lane Roads

Hughes et al. [1996] reviewed the crash databases of five states and showed that vehicleanimal crashes had increased almost 70\% between 1985 and 1991. It was shown that in one state, such crashes accounted for over 33\% of all reported crashes on rural two-lane roads. That study also found that vehicle-animal crashes are increasing at a rate that exceeds that of other crash causes. Fitzpatrick et al. [2000] explains that such trends could be due to continuing development and changing land use patterns, increases in deer populations, and increases in traffic volume at areas populated by deer. It is expected that the trends will continue to increase, at least in the near future. Hughes et al. [1996] also found that most vehicle-animal crashes occurred in the early hours of the morning (5 - 8AM) and at night (6PM to midnight), and that most occurred in October and November (which is the deer mating season). 
Deer related crashes may be reduced using signing, improvements to roadside vegetation, and reflectors to redirect the light from vehicle headlights into the neighboring terrain. In Indiana, advance deer crossing warning signs can be found at many road sections where significant deer activity is expected. Ward [1982] reported that a high game fence was constructed along an interstate and deer food was placed at specific locations to control the movement of deer. In a similar study carried out in Utah, it was determined that some deer collisions may be avoided by placing food at points away from the highways [IIHS, 1986] as such feeding areas intercepted foraging deer and kept them away from the highways. In some cases, reflective devices have been installed near the roadway to redirect the light from vehicle headlights as red lights to create "optical fences' to keep deer from crossing or entering the roadway. This is based on the premise that white tailed deer are afraid of the illuminated red reflectors and they therefore either stop or flee from highway when the reflectors are illuminated by an approaching vehicle [Zachs, 1986].

The effectiveness of some of these countermeasures have been investigated. The effectiveness of advance warning signs is unknown. Fitzpatrick et al. caution that overusing such signs may result in lack of driver's attention to the message. Ward [1982] and Ludwig and Bremicker [1983] reported that using fencing and underpasses has resulted in fewer deer crossing the roadways and fewer crashes. Also, Ludwig and Bremicker [1983] studied two road segments that received such countermeasures, and found that the reported number of deer hits was reduced by between 60 and 93\%. Schafer et al. [1985] and Zachs [1986] investigated the efficacy of reflective devices in reducing deer crashes and found that in some cases there was significant crash reduction, while in some cases, the impact was minimal as the deer reacted to the red light in ways that were unexpected.

\subsection{TRAFFIC CONTROL DEVICES}

While strictly considered a roadway characteristic, a traffic control device is nevertheless a fixed physical engineering facility that plays an important role in safety enhancement and merit attention in the present study. All else being the same, a well-informed driver or one made aware of imminent danger can respond appropriately to avert a potential crash situation. Traffic control devices provide the opportunity for quick delivery of information to the vehicle operator. The MUTCD provides guidelines on effective us of traffic control devices that can greatly enhance safety. Lack of or inadequacy of traffic control measures has been found to be a cause of road crashes in some cases. The effectiveness of traffic control devices could be evaluated by observing driver behavior with and 
without the installation of such devices. The three main countermeasures to a situation with little or no information delivery are: provision of signs, delineation, and pavement markings. It seems that past research on traffic control devices have focused on their effects on driver behavior than on crashes. 


\section{CHAPTER 7 CRASH PREDICTION MODELS, CRASH REDUCTION FACTORS, AND COST-EFFECTIVENESS RATIOS}

\subsection{CRASH PREDICTION MODELS}

This section presents and discusses the results of the crash prediction modeling for the rural two-lane roads on the Indiana's state highway and county road systems. The engineering factors that affect crash frequency on these roads are identified and the magnitudes of their impacts were estimated.

\subsubsection{Crash Prediction Models for Indiana's Rural Two-lane State Highways}

For the present study, three classes of state highway rural two-lane roads were considered: rural major collectors, rural minor arterials and rural principal arterials. This classification reflects the significant differences in the interactive effects of mobility and accessibility that are not explicitly addressed by quantitative variables. Also, the classification levels serve as qualitative variables that surrogate for certain traffic characteristics and geometric design standards which were not included in the modeling process due to lack of data or other reasons. Such variables include truck percentage, speed limits, speed variance, access control, etc.

For each functional class, four crash severity were modeled -- Injury crashes, PDO crashes, Fatal/Injury crashes and Total crashes. Details of the modeling results are presented in Appendix D. The next paragraphs discuss the general results of all the models, on a variable-by-variable basis.

The Section Length variable, L_LENG (expressed as the natural logarithm of the road section length) was consistently found to be significant at the specified degree of confidence, with positive coefficients in all the crash models. This relationship is rather obvious, for there is no question that longer road sections are expected to have more crashes compared to shorter ones, all other factors being the same. The main issue, rather, is the nature of the relationship between crash frequency and section length - is Section Length linearly related to crash frequency? This was investigated using Wald's test for linear restrictions. This test ascertains whether the index associated with the Section Length variable is significantly different from one. The model results showed that with the exception of the Total and PDO crash models for the rural principal arterials, the index of the Section Length variable was not significantly different from one confirming that the relationship between the number of crashes and section lengths may generally be considered linear. In other words, doubling the section 
length on a road, for instance, may generally be expected to yield a doubled number of crashes - the only exception being PDO crashes at rural principal arterials where less-than-double crashes may be expected.

As expected, the Traffic Volume variable $L_{-} A A D T$, (expressed as the natural logarithm of the traffic volume variable) turned out to be a significant variable in all the crash models for all road classes: increased traffic volume is associated with greater frequency of crashes, which is consistent with expectation at least within the range of traffic volumes typically encountered in rural two-lane roads. The relationship between traffic volume and number of crashes was investigated to determine whether the number of crashes is linearly related to traffic volume using the Wald's test for linear restrictions. The results of the test indicated that the index associated with the Traffic Volume variable was significantly different from unity for all the crash models thus supporting the general premise that the relationship between traffic volume and number of crashes is non linear. In other words, doubling the traffic volume of a rural two-lane road in Indiana for instance, is not expected to yield a doubled number of crashes. This finding seems quite intuitive, because increasing congestion may lead to lower speeds (which could result in reduced crashes) but may also lead to higher speed variance (which could lead to increased crashes), and the net effect could go either way.

The modeling results also showed that the Lane Width variable, $L W$, was found to be a significant factor in all the crash models. The negative coefficient for the lane width in all the models indicates that wider lane widths are associated with lower crashes. This result is consistent with expectation because wider lanes serve as buffer zones offering more opportunity for errant vehicles to recover or for vehicles to seek temporary refuge to avoid an errant oncoming vehicle and therefore reduce the risk of collision.

The Shoulder Width variable was shown to have a significant influence on crashes, a consistent result for all road classes and crash severity types. The negative sign of the coefficient suggests that for rural two-lane roads, increasing shoulder width is associated with decreasing in number of crashes, all other factors remaining the same. This result is expected and can be explained by the fact that for rural two-lane roads, wider shoulders enhance safety by providing additional buffer zone where operators of stray vehicles can regain control, recover from error and resume normal travel. The model results also suggest that the impact of increasing shoulder width is greater for injury crash frequency compared to non-injury crash frequency.

Pavement Friction, FR, was found to be a significant variable for the relatively lower classes of roads -- rural major collectors and minor arterials. The negative sign of the Pavement Friction coefficient indicates that sections with higher pavement friction experience lower rates. This is an 
expected result since higher pavement friction reduces the vehicle stopping distance thus reducing the occurrence especially rear-end crashes. The effect of pavement friction however, was found to be insignificant in the crash models for the highest class of rural two-lane roads (principal arterials) possibly due to the lack of variability of that variable for such class of roads.

The model results also showed that Pavement Condition, expressed as the present serviceability index, $S I$, is significant for rural principal arterials, but not for the two lower road classes. PSI values recorded in the inventory database ranges between 2.5 and 5.0. Higher PSI values indicate better pavement condition. The analysis results indicate that roads with superior pavement conditions seem to experience fewer crashes. This finding seems to be intuitive for many reasons. For example, drivers seeking to avoid a surface defect may stray onto the adjacent lane (into the path of oncoming vehicles) or may stray onto the roadside where they may strike obstacles or roll over a steep embankment.

It was determined that the Average Horizontal Curve Radius $(A R A D)$ was a significant factor in all the crash models. This finding suggests that sections with higher average curve radius experience fewer crashes, which is consistent with expectation. A wider horizontal curve radius provides smoother transition between tangent section and leads to reduction of centrifugal forces on vehicles negotiating the curve, thereby reducing the risk of overturning. The effect of Average Vertical Grade $(A V \mathrm{R} A D)$ was also found to be significant for all crash severity types and all road classes. The positive sign associated with this variable indicates that sections with lower average vertical grades experience fewer crashes. This is an expected result since lower vertical grades improves sight distance and enhances safety.

A summary of the crash prediction equations for the rural two-lane state highways is provided below. $L N(N)$ refers to the natural logarithm of crash frequency.

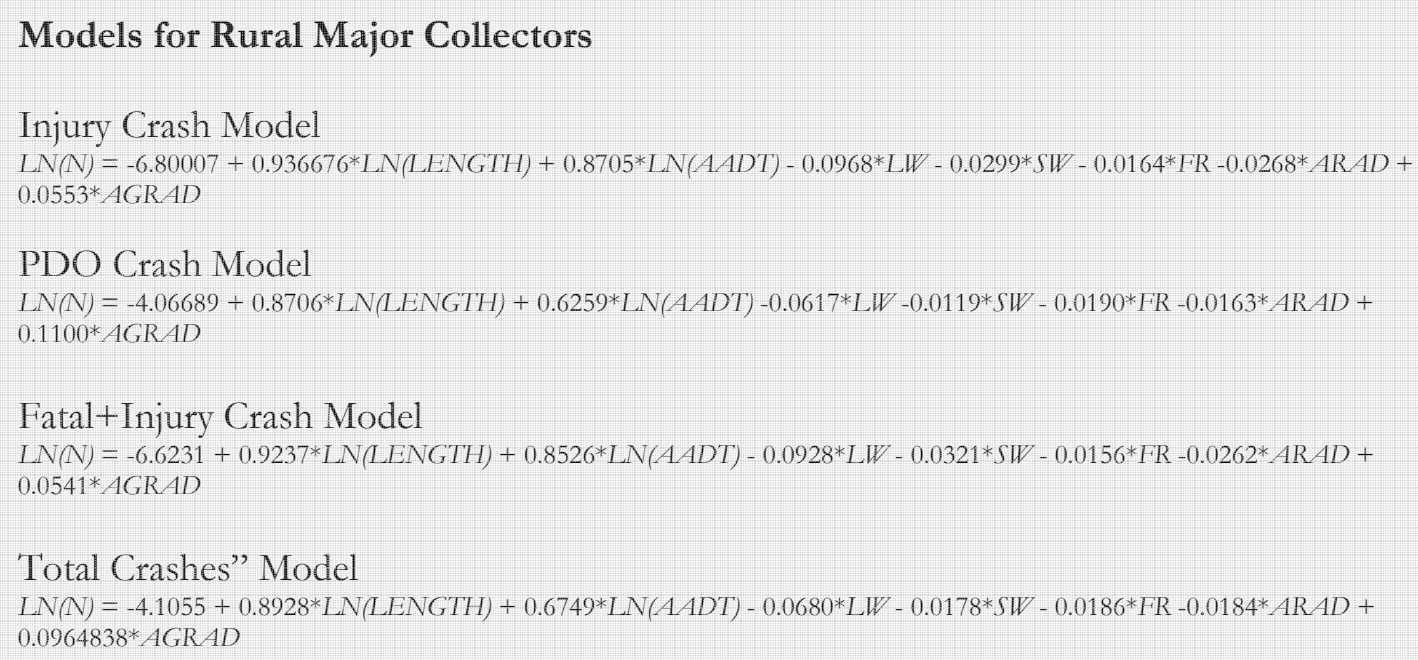


repeated with variables that were found significant. Then a second model was developed using only the observations that had a shoulder width. The next section discusses the general results of all the models, on a variable-by-variable basis.

The Section Length variable, $L \_L E N G$, was consistently found to be significant, with a positive coefficient. This is expected because longer county road sections are expected to have a higher number of more crashes compared to shorter ones, all else being equal. As expected, Traffic Volume (L_AADT) turned out to be a significant variable in all the crash models for all road classes: Higher AADT is associated with higher crash frequency. It was also seen that the Lane Width variable, $L W$, was found to be a significant factor, and has an indirect relationship with crash frequency: wider lane widths are associated with fewer crashes. This is an expected result as wider lanes offer additional buffer zones for vehicle recovery. The Shoulder Type variable, RST, was shown to have a significant influence on crashes at county roads. Increasing quality of shoulder (from no shoulder to gravel/grass to asphalt) is associated with decreasing crash frequency, as reflected in the negative coefficient. This result is expected because of the better grip of vehicle tires at treated shoulder surfaces compared to untreated shoulder surfaces. The model results also showed that Driveway Density, $D D$, is an influential factor affecting crashes at county roads. From the model results, it is seen that a greater number of driveways per mile is associated with higher crash frequency. This is consistent with expectation and past findings. It was determined that the posting of speed limits, SPL, served to reduce the frequency of crashes, as seen from the model results. This seems to be contrary to expectation, as the mere presence of such postings is expected to engender more cautious driving. On the other hand, it may be argued that the speed limit posting at county roads are generally higher than speeds that are consistent with their existing geometric and other safety features, and that drivers on county roads generally tend to be less cautious in the absence of a speed limit and more cautious when a speed limit sign is present. The value of the posted speed was not considered. Horizontal Curve Density, $H C D$, was a significant factor in all the county roads crash model. The model results suggest that sections with a higher number of horizontal curves experience higher crash frequency, all else being equal. This is consistent with expectation.

The model with only 10 observations that included Shoulder Width showed that increasing shoulder width is associated with decreasing crash frequency, all other factors remaining the same.

The crash prediction models for the county roads are presented as follows: 


\section{County Roads}

Model for County Road Crashes Excluding Shoulder Width Variable

$L N(N)=-1.45459-0.336618 * \operatorname{LN}(L E N G T H)+0.825750 * \operatorname{LN}(A A D T)-0.358197 * L W-0.412005 * S T+0.0191902 * D D+0.571718$ $* S P L+0.571079 * H C D-0.448448 *$ RST

Model for County Road Crashes Including Shoulder Width Variable

$L N(N)=-5.00725-0.112947 * \operatorname{LN}(L E N G T H)+0.833491 * \operatorname{LN}(A A D T)-0.181543 * S W$

\subsection{DEVELOPMENT OF CRASH REDUCTION FACTORS (STATISTICAL ESTIMATION OF EFFECTIVENESS OF SAFETY COUNTERMEASURES)}

The cross-sectional approach was used to develop crash prediction models which were in turn used to develop crash reduction factors for each countermeasure, crash severity type, and road class. Specifically, the crash reduction factors were developed for:

Rural Minor Arterials and Rural Major Collectors:

- CRF's for Lane Widening

- CRF's for Shoulder Widening

- CRF's for Friction Number Improving

- CRF's for Horizontal Curve Widening

- CRF's for Vertical Grade Flattening

Rural Principal Arterials

- CRF's for Lane Widening

- CRF's for Shoulder Widening

- CRF's for Pavement Condition Improvement

- CRF's for Horizontal Curve Widening

- CRF's for Vertical Grade Flattening

County Roads

- CRFs for Lane Widening

- CRFs for Shoulder Widening

- CRFs for Shoulder Paving

- CRFs for Driveway Access Restriction,

- CRFs for Horizontal Curve Widening

- CRFs for Road Surface Stabilization (Paving)

The results are presented in the figures and tables shown in the following section. 


\subsubsection{Crash Reduction Factors for Safety Enhancements at Rural Major Collectors}

Using the crash prediction models developed as part of the present study, (Section 7.1), crash reduction factors were then developed for each countermeasure.

\subsubsection{Crash Reduction by Extent of Countermeasure}

As seen from Figure 7-1, increasing lane width and shoulder width of rural major collector roads is associated with decreasing crashes, for all crash severity types. This is intuitive because with increasing lane and shoulder widths, errant vehicles are provided a greater opportunity to recover their driving paths thus reducing the likelihood of crash occurrence. These countermeasures seem to be most effective for fatal and injury crashes, and relatively less effective for PDO crashes. Also, the results suggest that fatal and injury crash reductions seem to be more sensitive to increases in lane width or shoulder width compared to PDO crash reductions. Increasing friction number of rural major collector roads (this is typically achieved through surface treatments such as micro-surfacing) is associated with greater crash reduction, for all crash types. The magnitude of crash reduction and the sensitivities thereof appear to be fairly uniform across crash severity types. This is consistent with expectation because with increased friction, the skid resistance of vehicle tires is enhanced and drivers are more able to slow down or to bring their vehicles to a stop before encountering a possible crash. The effectiveness trends for increasing horizontal curve radius seem to be similar to those for laneand shoulder widening: Increasing radius is associated with increasing crash reduction, and such effectiveness is higher (and also more sensitive) for fatal and injury crashes, and is lower for PDO crashes. For increasing quality of vertical alignment (decreasing vertical grade), a greater crash reduction is observed. This is expected because sight distances are increased through such improvements. However, unlike the cases for other countermeasures, the effectiveness of vertical grade improvements seems to be higher for PDO crashes and lower for fatal and injury crashes. Also, PDO crash reduction appears to be more sensitive to decreases in vertical grade, compared to fatal and injury crashes. The results are consistent with the preliminary descriptive statistics presented in Chapter 6.

\subsubsection{Crash Reduction by Type of Countermeasure}

It may not be very meaningful to compare the effectiveness across different countermeasures except in the following situations: (i) the countermeasures are viable alternative actions to remedy a single safety problem at an existing road, or (ii) an existing road suffers from multiple types of safety 
problems so many different countermeasures may be carried out and fund are available to carry out relatively few of such countermeasures. Figure 7-1 suggests that enhancements in vertical alignment seem to have the highest crash reductions (approximately 80\% for total crashes for a decrease in vertical grade by $6 \%$ ). Increase in friction number by 5 units affords $8-9 \%$ crash reductions, while a 5 -mile increase in horizontal curve radius generally yields a $7-13 \%$ crash reduction depending on crash severity type. Increases in lane-widths seem to be more effective than increases in shoulder width: a $4 \mathrm{ft}$ increase in lane width provides $22-32 \%$ crash reduction, while a similar increase in shoulder width yields a $5-12 \%$ crash reduction.

\subsubsection{Crash Reduction by Crash Severity Type}

The results shown in Figure 7-1 suggest that increases in lane width and shoulder width, and increases in horizontal curve radius yield greater reductions in fatal and injury crashes and relatively smaller reductions in PDO crashes, all other factors being the same. Also, fatal and injury crashes are more sensitive to increases in lane and shoulder width, and horizontal curve radius On the other hand, for improvements in vertical alignment (and, to a smaller extent, enhanced pavement skid resistance), it is

seen that PDO crashes have a greater magnitude of crash reduction and greater sensitivity compared to fatal and injury crashes. The findings also suggest that as the level of countermeasure (extent of the improvement) increases, there is increasing divergence of crash reduction across crash severity types. Such divergence is most perceptible in the case of vertical grade improvements, and least perceptible for increase in friction number. 

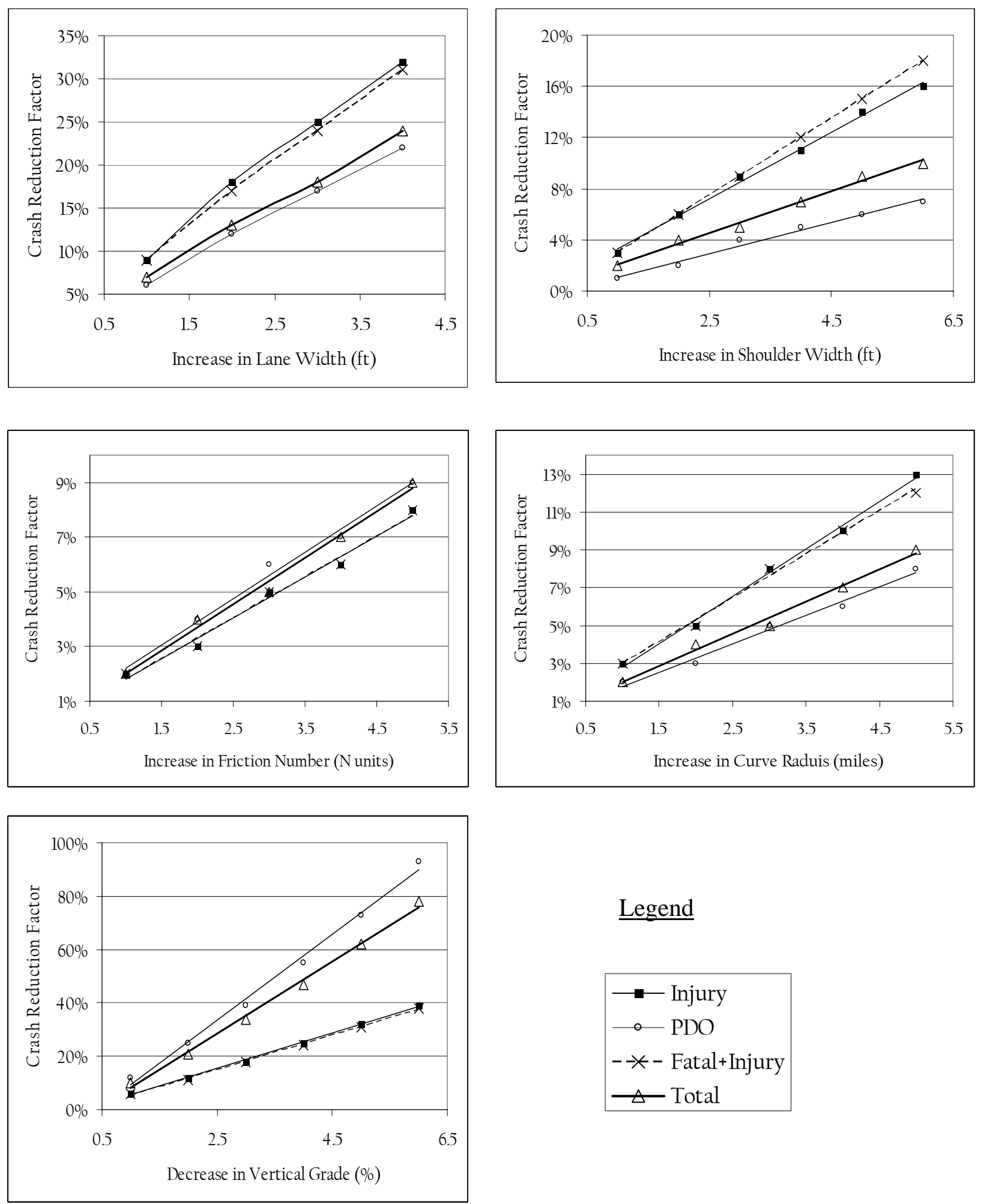

\section{$\underline{\text { Legend }}$}

$\longrightarrow-$ Injury
- PDO
$--x-$ Fatal+Injury
$\triangle-$ Total

Figure 7-1: Crash Reduction Factors by Improvement Type and Crash Type, Rural Major Collectors 
Table 7-1: Crash Reduction Factors for Various Safety Improvements at Rural Major Collectors

(a) Lane and Shoulder Widening

\begin{tabular}{|c|c|c|c|c|}
\hline $\begin{array}{c}\text { Increase in Lane } \\
\text { Width (ft) } \\
1\end{array}$ & Injury & PDO & Fatinj & Total \\
\cline { 2 - 5 } & $9 \%$ & $6 \%$ & $9 \%$ & $7 \%$ \\
\hline 2 & $18 \%$ & $12 \%$ & $17 \%$ & $13 \%$ \\
\hline 3 & $25 \%$ & $17 \%$ & $24 \%$ & $18 \%$ \\
\hline 4 & $32 \%$ & $22 \%$ & $31 \%$ & $24 \%$ \\
\hline
\end{tabular}

\begin{tabular}{|c|c|c|c|c|}
\hline $\begin{array}{c}\text { Increase in Shoulder } \\
\text { Width (ft) }\end{array}$ & Injury & PDO & Fatinj & Total \\
\cline { 2 - 5 } & $3 \%$ & $1 \%$ & $3 \%$ & $2 \%$ \\
\hline 2 & $6 \%$ & $2 \%$ & $6 \%$ & $4 \%$ \\
\hline 3 & $9 \%$ & $4 \%$ & $9 \%$ & $5 \%$ \\
\hline 4 & $11 \%$ & $5 \%$ & $12 \%$ & $7 \%$ \\
\hline 5 & $14 \%$ & $6 \%$ & $15 \%$ & $9 \%$ \\
\hline 6 & $16 \%$ & $7 \%$ & $18 \%$ & $10 \%$ \\
\hline
\end{tabular}

(b) Skid Resistance Improvement and Horizontal Curve Widening

\begin{tabular}{|c|c|c|c|c|}
\hline $\begin{array}{c}\text { Increase in } \\
\text { Friction Number } \\
1\end{array}$ & Injury & PDO & Fatinj & Total \\
\cline { 2 - 5 } & $2 \%$ & $2 \%$ & $2 \%$ & $2 \%$ \\
\hline 2 & $3 \%$ & $4 \%$ & $3 \%$ & $4 \%$ \\
\hline 3 & $5 \%$ & $6 \%$ & $5 \%$ & $5 \%$ \\
\hline 4 & $6 \%$ & $7 \%$ & $6 \%$ & $7 \%$ \\
\hline 5 & $8 \%$ & $9 \%$ & $8 \%$ & $9 \%$ \\
\hline
\end{tabular}

\begin{tabular}{|c|c|c|c|c|}
\hline $\begin{array}{c}\text { Increase in Avg. Horizontal } \\
\text { Curve Radius (mile) }\end{array}$ & Injury & PDO & Fatinj & Total \\
\cline { 2 - 5 } & $3 \%$ & $2 \%$ & $3 \%$ & $2 \%$ \\
\hline 2 & $5 \%$ & $3 \%$ & $5 \%$ & $4 \%$ \\
\hline 3 & $8 \%$ & $5 \%$ & $8 \%$ & $5 \%$ \\
\hline 4 & $10 \%$ & $6 \%$ & $10 \%$ & $7 \%$ \\
\hline 5 & $13 \%$ & $8 \%$ & $12 \%$ & $9 \%$ \\
\hline
\end{tabular}

(c) Vertical Grade Improvement

\begin{tabular}{|c|c|c|c|c|}
\hline $\begin{array}{c}\text { Decrease in Avg. } \\
\text { Vertical Curve Grade } \\
(\mathbf{\%})\end{array}$ & Injury & PDO & Fatinj & Total \\
\cline { 2 - 5 } 1 & $6 \%$ & $12 \%$ & $6 \%$ & $10 \%$ \\
\hline 2 & $12 \%$ & $25 \%$ & $11 \%$ & $21 \%$ \\
\hline 3 & $18 \%$ & $39 \%$ & $18 \%$ & $34 \%$ \\
\hline 4 & $25 \%$ & $55 \%$ & $24 \%$ & $47 \%$ \\
\hline 5 & $32 \%$ & $73 \%$ & $31 \%$ & $62 \%$ \\
\hline 6 & $39 \%$ & $93 \%$ & $38 \%$ & $78 \%$ \\
\hline
\end{tabular}




\subsubsection{Crash Reduction Factors for Safety Enhancements at Rural Minor Arterials}

\subsubsection{Crash Reduction by Extent of Countermeasure}

As seen from Figure 7-2, increasing lane width, shoulder width and horizontal curve radius of rural minor arterials, like their major collector counterparts, are associated with greater crash reduction, for all crash severity types. Also, increases in friction number of rural minor arterials are associated with greater crash reduction, for all crash types. For increasing quality of vertical alignment (decreasing vertical grade), a greater crash reduction is observed. These findings are consistent with the preliminary descriptive statistics presented in Chapter 6.

\subsubsection{Crash Reduction by Type of Countermeasure}

For rural minor arterial road sections, the findings for crash reduction by countermeasure type were generally similar to those for rural major collectors. Figure 7-2 suggests that enhancements in vertical alignment seem to have the highest crash reductions (up to approximately $80 \%$ for total crashes for a decrease in vertical grade by $6 \%$ ). Increase in friction number by 5 units affords $6-15 \%$ crash reductions, while a 5-mile increase in horizontal curve radius generally yields a $25-32 \%$ crash reduction depending on crash severity type. The findings suggest that increases in lane width are more effective than increases in shoulder width: a $4 \mathrm{ft}$ increase in lane width provides $30-32 \%$ crash reduction, while a similar increase in shoulder width yields a $12-14 \%$ crash reduction.

\subsubsection{Crash Reduction by Crash Severity Type}

Figure 7-2 suggests that increases in shoulder width and skid resistance yield greater reductions in fatal and injury crashes and relatively smaller reductions in PDO crashes, all other factors being the same. Also, fatal and injury crashes are more sensitive to increases in shoulder width and skid resistance. On the other hand, for improvements in horizontal curve alignment, it is seen that PDO crashes have a greater magnitude of crash reduction and greater sensitivity compared to fatal and injury crashes. For increases in lane width and decrease in vertical grade, there seemed to be little or no differences in crash reduction across crash severity types. The findings also suggest that as the level of countermeasure (extent of the improvement) increases, there is increasing divergence of effectiveness (crash reduction) across crash severity types. For rural minor arterials, such divergence is most perceptible in the case of increase in skid resistance and least perceptible for increase in lane width. 

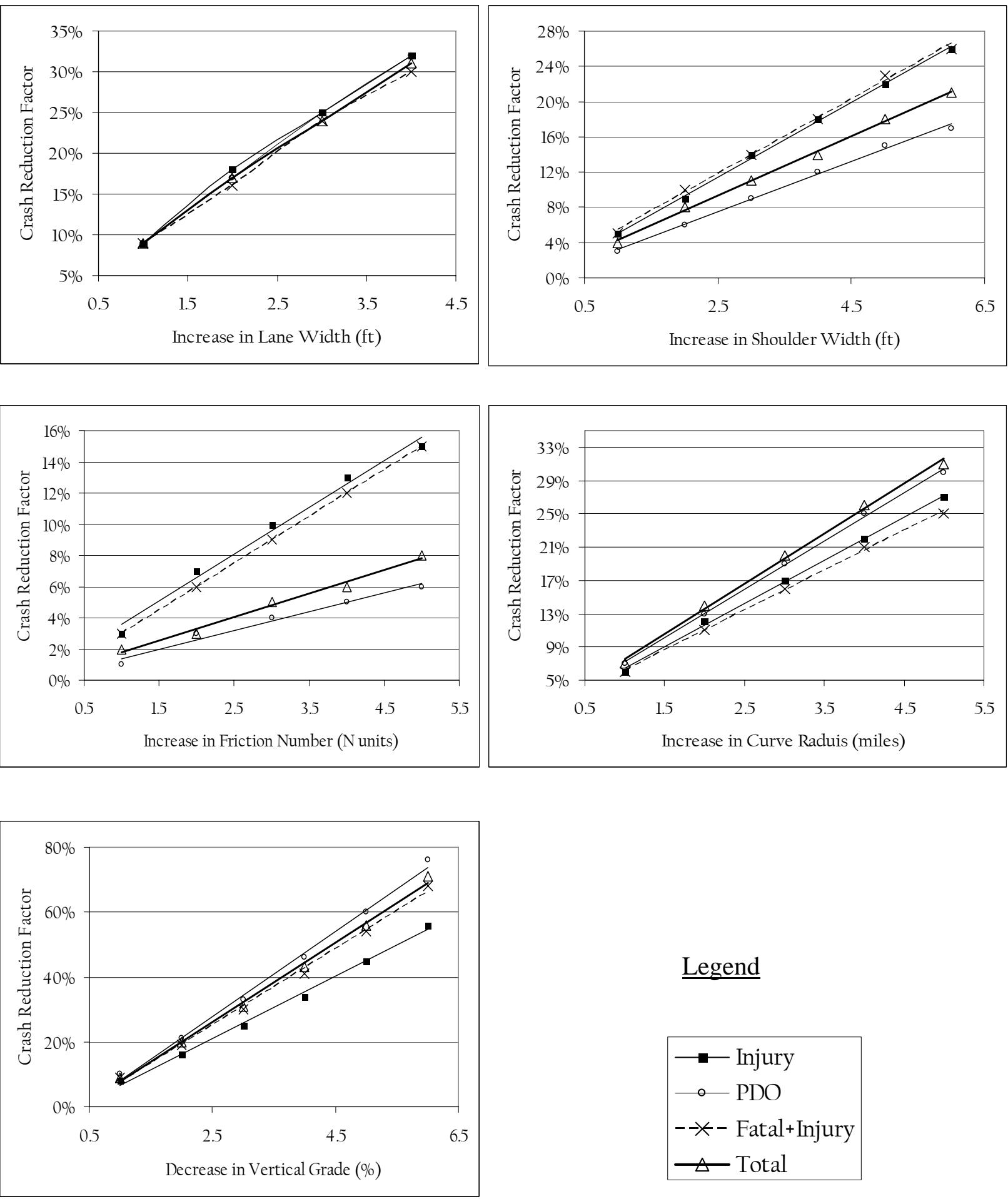

\section{$\underline{\text { Legend }}$}

$\longrightarrow-$ Injury
-- PDO
$--\star-$ Fatal+Injury
$\triangle$ Total

Figure 7-2: Crash Reduction Factors by Improvement Type and Crash Type, Rural Minor Arterials 
Table 7-2: Crash Reduction Factors for Various Safety Improvements at Rural Minor Arterials

(a) Lane Widening and Shoulder Widening

\begin{tabular}{|c|c|c|c|c|}
\hline $\begin{array}{c}\text { Increase in Lane } \\
\text { Width (ft) }\end{array}$ & Injury & PDO & Fatinj & Total \\
\cline { 2 - 5 } & $9 \%$ & $9 \%$ & $9 \%$ & $9 \%$ \\
\hline 2 & $18 \%$ & $17 \%$ & $16 \%$ & $17 \%$ \\
\hline 3 & $25 \%$ & $25 \%$ & $24 \%$ & $24 \%$ \\
\hline 4 & $32 \%$ & $32 \%$ & $30 \%$ & $31 \%$ \\
\hline
\end{tabular}

\begin{tabular}{|c|c|c|c|c|}
\hline $\begin{array}{c}\text { Increase in Shoulder } \\
\text { Width (ft) }\end{array}$ & Injury & PDO & Fatinj & Total \\
\cline { 2 - 5 } & $5 \%$ & $3 \%$ & $5 \%$ & $4 \%$ \\
\hline 2 & $9 \%$ & $6 \%$ & $10 \%$ & $8 \%$ \\
\hline 3 & $14 \%$ & $9 \%$ & $14 \%$ & $11 \%$ \\
\hline 4 & $18 \%$ & $12 \%$ & $18 \%$ & $14 \%$ \\
\hline 5 & $22 \%$ & $15 \%$ & $23 \%$ & $18 \%$ \\
\hline 6 & $26 \%$ & $17 \%$ & $26 \%$ & $21 \%$ \\
\hline
\end{tabular}

(b) Skid Resistance and Horizontal Alignment Improvements

\begin{tabular}{|c|c|c|c|c|}
\hline $\begin{array}{c}\text { Increase in } \\
\text { Friction Number }\end{array}$ & Injury & PDO & Fatinj & Total \\
\cline { 2 - 5 } & $3 \%$ & $1 \%$ & $3 \%$ & $2 \%$ \\
\hline 2 & $7 \%$ & $3 \%$ & $6 \%$ & $3 \%$ \\
\hline 3 & $10 \%$ & $4 \%$ & $9 \%$ & $5 \%$ \\
\hline 4 & $13 \%$ & $5 \%$ & $12 \%$ & $6 \%$ \\
\hline 5 & $15 \%$ & $6 \%$ & $15 \%$ & $8 \%$ \\
\hline
\end{tabular}

\begin{tabular}{|c|c|c|c|c|}
\hline $\begin{array}{c}\text { Increase in Avg. Horizontal } \\
\text { Curve Radius (mile) }\end{array}$ & Injury & PDO & Fatinj & Total \\
\cline { 2 - 5 } & $6 \%$ & $7 \%$ & $6 \%$ & $7 \%$ \\
\hline 2 & $12 \%$ & $13 \%$ & $11 \%$ & $14 \%$ \\
\hline 3 & $17 \%$ & $19 \%$ & $16 \%$ & $20 \%$ \\
\hline 4 & $22 \%$ & $25 \%$ & $21 \%$ & $26 \%$ \\
\hline 5 & $27 \%$ & $30 \%$ & $25 \%$ & $31 \%$ \\
\hline
\end{tabular}

(c) Vertical Grade Improvement

\begin{tabular}{|c|c|c|c|c|}
\hline $\begin{array}{c}\text { Decrease in Avg. Vertical } \\
\text { Curve Grade (\%) }\end{array}$ & Injury & PDO & Fatinj & Total \\
\cline { 2 - 5 } & $8 \%$ & $10 \%$ & $9 \%$ & $9 \%$ \\
\hline 2 & $16 \%$ & $21 \%$ & $19 \%$ & $20 \%$ \\
\hline 3 & $25 \%$ & $33 \%$ & $30 \%$ & $31 \%$ \\
\hline 4 & $34 \%$ & $46 \%$ & $41 \%$ & $43 \%$ \\
\hline 5 & $45 \%$ & $60 \%$ & $54 \%$ & $56 \%$ \\
\hline 6 & $56 \%$ & $76 \%$ & $68 \%$ & $71 \%$ \\
\hline
\end{tabular}




\subsubsection{Crash Reduction Factors for Safety Enhancements at Rural Principal Arterials}

\subsubsection{Crash Reduction by Extent of Countermeasure}

As seen from Figure 7-3, increasing shoulder width, horizontal curve radius, skid resistance, and quality of vertical alignment (decreasing vertical grade) of rural principal arterials, like their major collector and minor arterial counterparts, are associated with greater crash reduction, for all crash severity types. For this class of roads, however, increases in shoulder width lead to increasing crash reduction only up to a point - beyond $5 \mathrm{ft}$ of shoulder widening, the crash reduction factor drops sharply, for all crash severity types.

\subsubsection{Crash Reduction by Type of Countermeasure}

For rural principal arterials, Figure 7-3 suggests that enhancements in vertical alignment seem to have the highest crash reductions (up to approximately $40 \%$ for total crashes for a decrease in vertical grade by $6 \%$ ). Increase in pavement condition by 2.5 PSI units affords $15-40 \%$ crash reductions depending on crash severity type, while a 5 -mile increase in horizontal curve radius generally yields a $16-24 \%$ crash reduction depending on crash severity type. The findings suggest that for PDO crashes, increases in lane width are more effective than increases in shoulder width: a $4 \mathrm{ft}$ increase in lane width provides 35\% PDO crash reduction, while a similar increase in shoulder width yields a $24 \%$ PDO crash reduction. On the other hand, for fatal and injury crashes, increases in lane width are less effective than increases in shoulder width: a $4 \mathrm{ft}$ increase in lane width provides $28 \%$ fatal+injury crash reduction, while a similar increase in shoulder width yields a 31\% crash reduction. However, increases in shoulder width by $6 \mathrm{ft}$ or more is less effective than similar increases in lane width for all crashes.

\subsubsection{Crash Reduction by Crash Severity Type}

Figure 7-3 suggests that increases in shoulder width and pavement condition at rural principal arterials yield greater reductions in fatal and injury crashes and relatively smaller reductions in PDO crashes, all other factors being the same. Also, the figures show that fatal and injury crashes are more sensitive to increases in shoulder width and pavement condition. On the other hand, for increases in lane width and improvements in horizontal and vertical alignments, it is seen that PDO crashes have a greater magnitude of crash reduction and greater sensitivity compared to fatal and injury crashes. It is also seen for all crash severity types that there is increasing divergence of effectiveness (crash reduction) as the level of countermeasure (extent of the improvement) increases. For rural principal arterials, such divergence is most perceptible in the case of increase in pavement condition. 

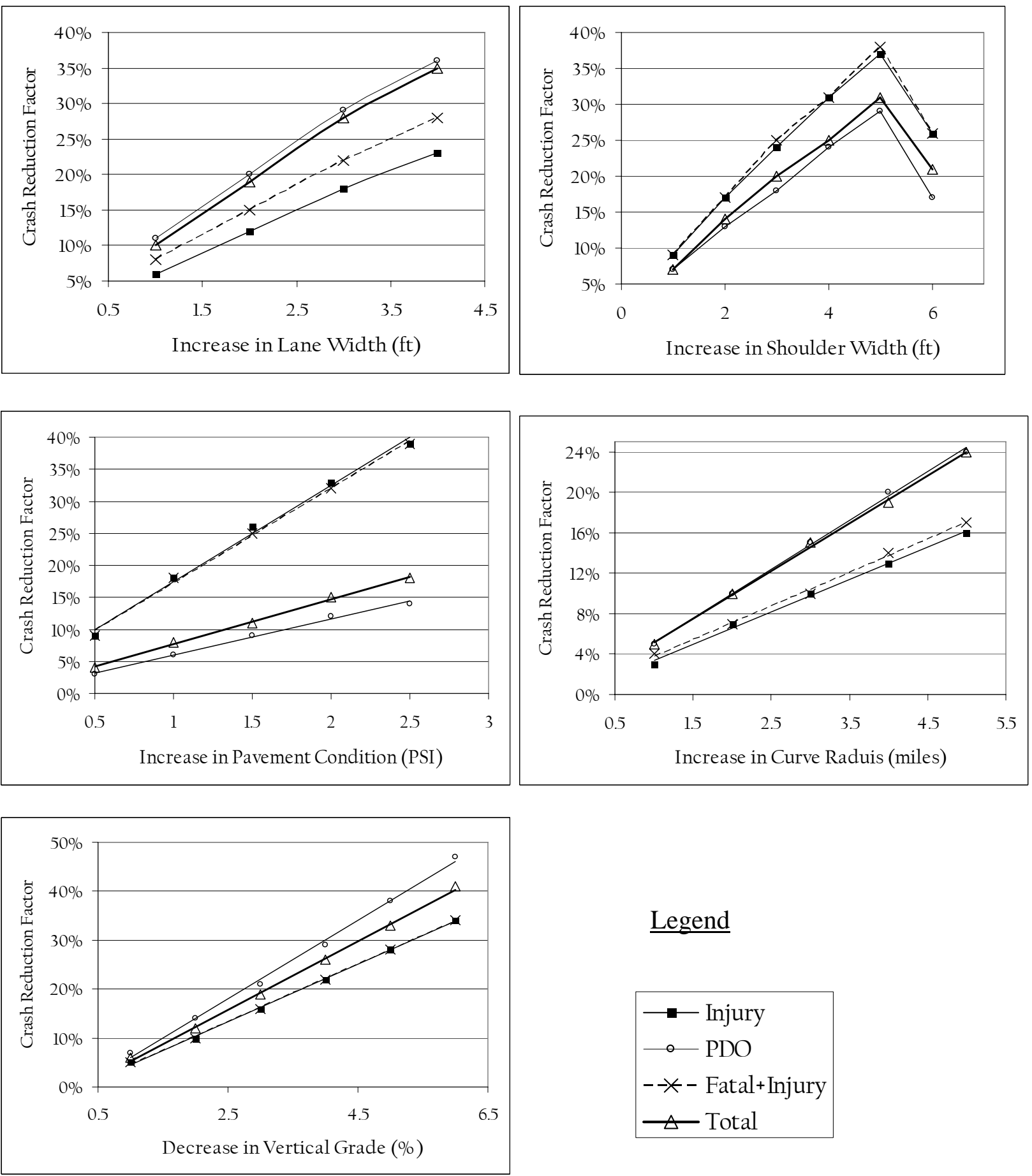

Figure 7-3: Crash Reduction Factors by Improvement Type and Crash Type, Rural Principal Arterials 
Table 7-3: Crash Reduction Factors for Various Safety Improvements at Rural Principal Arterials

(a) Lane Widening and Shoulder Widening

\begin{tabular}{|c|c|c|c|c|}
\hline $\begin{array}{c}\text { Increase in Lane } \\
\text { Width (ft) }\end{array}$ & Injury & PDO & Fatinj & Total \\
\cline { 2 - 5 } & $6 \%$ & $11 \%$ & $8 \%$ & $10 \%$ \\
\hline 2 & $12 \%$ & $20 \%$ & $15 \%$ & $19 \%$ \\
\hline 3 & $18 \%$ & $29 \%$ & $22 \%$ & $28 \%$ \\
\hline 4 & $23 \%$ & $36 \%$ & $28 \%$ & $35 \%$ \\
\hline
\end{tabular}

\begin{tabular}{|c|c|c|c|c|}
\hline $\begin{array}{c}\text { Increase in Shoulder } \\
\text { Width (ft) }\end{array}$ & Injury & PDO & Fatinj & Total \\
\cline { 2 - 5 } & $9 \%$ & $7 \%$ & $9 \%$ & $7 \%$ \\
\hline 2 & $17 \%$ & $13 \%$ & $17 \%$ & $14 \%$ \\
\hline 3 & $24 \%$ & $18 \%$ & $25 \%$ & $20 \%$ \\
\hline 4 & $31 \%$ & $24 \%$ & $31 \%$ & $25 \%$ \\
\hline 5 & $37 \%$ & $29 \%$ & $38 \%$ & $31 \%$ \\
\hline 6 & $26 \%$ & $17 \%$ & $26 \%$ & $21 \%$ \\
\hline
\end{tabular}

(b) Pavement Condition Improvement

\begin{tabular}{|c|c|c|c|c|}
\hline $\begin{array}{c}\text { Increase in Pavement } \\
\text { Serviceability Index } \\
0.5\end{array}$ & Injury & PDO & Fatinj & Total \\
\cline { 2 - 5 } & $9 \%$ & $3 \%$ & $9 \%$ & $4 \%$ \\
\hline 1 & $18 \%$ & $6 \%$ & $18 \%$ & $8 \%$ \\
\hline 1.5 & $26 \%$ & $9 \%$ & $25 \%$ & $11 \%$ \\
\hline 2 & $33 \%$ & $12 \%$ & $32 \%$ & $15 \%$ \\
\hline 2.5 & $39 \%$ & $14 \%$ & $39 \%$ & $18 \%$ \\
\hline
\end{tabular}

(c)Horizontal Curve and Vertical Alignment Improvements

\begin{tabular}{|c|c|c|c|c|}
\hline $\begin{array}{c}\text { Increase in Avg. Horizontal } \\
\text { Curve Radius (mile) }\end{array}$ & Injury & PDO & Fatinj & Total \\
\cline { 2 - 5 } & $3 \%$ & $5 \%$ & $4 \%$ & $5 \%$ \\
\hline 2 & $7 \%$ & $10 \%$ & $7 \%$ & $10 \%$ \\
\hline 3 & $10 \%$ & $15 \%$ & $10 \%$ & $15 \%$ \\
\hline 4 & $13 \%$ & $20 \%$ & $14 \%$ & $19 \%$ \\
\hline 5 & $16 \%$ & $24 \%$ & $17 \%$ & $24 \%$ \\
\hline
\end{tabular}

\begin{tabular}{|c|c|c|c|c|}
\hline $\begin{array}{c}\text { Decrease in Avg. Vertical } \\
\text { Curve Grade (\%) }\end{array}$ & Injury & PDO & Fatinj & Total \\
\cline { 2 - 5 } & $5 \%$ & $7 \%$ & $5 \%$ & $6 \%$ \\
\hline 2 & $10 \%$ & $14 \%$ & $10 \%$ & $12 \%$ \\
\hline 3 & $16 \%$ & $21 \%$ & $16 \%$ & $19 \%$ \\
\hline 4 & $22 \%$ & $29 \%$ & $22 \%$ & $26 \%$ \\
\hline 5 & $28 \%$ & $38 \%$ & $28 \%$ & $33 \%$ \\
\hline 6 & $34 \%$ & $47 \%$ & $34 \%$ & $41 \%$ \\
\hline
\end{tabular}




\subsubsection{Crash Reduction Factors for the Various Countermeasures at County Roads}

\subsubsection{Crash Reduction by Extent of Countermeasure}

Table 7-4 presents the crash reduction factors for county roads that were developed from the crash prediction equations.

Table 7-4: Crash Reduction Factors for County Roads

\begin{tabular}{|l|c|}
\hline Roadway characteristics & CRF \\
\hline Lane Width & $-30 \%$ \\
\hline Shoulder Width & $-16 \%$ \\
\hline Shoulder Type & $-34 \%$ \\
\hline Driveway Density & $2 \%$ \\
\hline H Curve Density & $77 \%$ \\
\hline Surface Type & $-36 \%$ \\
\hline
\end{tabular}

As seen in Table 7-4, a 100\% increase in lane width of county roads is associated with a 30\% reduction in crashes. Also, a 100\% increase in shoulder width is associated with a $16 \%$ reduction in crashes. It is seen that the impact of a unit change in lane width is greater than a unit change in shoulder width, a finding that is consistent with past research. Also, changing the shoulder type from No Shoulder to Grass or Gravel Shoulder leads to a 34\% reduction in crashes, and paving a grass shoulder offers a similar reduction in crashes. Decreasing the density of driveways through access restrictions would lead to a $2 \%$ reduction in crashes per every $100 \%$ decrease in access (expressed as the number of driveways per mile). However, it is doubtful whether such a measure would be practical in reality: dwellers at county roads, mostly farmers are likely to resist such governmental controls on access to their property. Decreasing the density of horizontal curves by straightening existing curves, while expensive, is expected to have far reaching impacts on crash reduction. Every $100 \%$ reduction in the number of curves per mile would yield $77 \%$ reduction in crashes. 


\subsubsection{General Discussion of Results across Road Functional Classes}

The four road functional classes considered in the present study (rural major collectors, rural minor arterials, rural principal arterials, and rural county roads) differ in their physical and operating characteristics. Such differences are evident in their levels of mobility (operating speeds), accessibility (density of intersections), percentage of trucks, levels of congestion, etc. While such individual variables are vital for crash prediction modeling, they were not directly used in the present study, but rather were surrogated by these functional class categories. Each functional class is characterized by a unique combination of these variables. A comparison of the crash reduction factors across the four functional classes revealed interesting results.

Lane Width: For PDO crashes, increase in lane width is most effective at rural principal arterials (e.g., $35 \%$ reduction for $4 \mathrm{ft}$ lane width increase) but least effective in at rural major collectors $(22 \%)$. On the other hand, for fatal and injury crashes, increase in lane width is most effective at rural major collectors (e.g., 31\% reduction in $4 \mathrm{ft}$ lane width increase) but least effective for rural principal arterials $(26 \%)$. It can therefore be seen that as the class of road decreases, relatively higher percentages of severe crashes and relatively lower percentage of non-severe crashes, are saved by increases in lane width. These findings were for the three functional classes on the state highway system. For county roads that lie at the bottom of the road classes, the high value of $30 \%$ crash reduction factor seems consistent with the observation that high crash reductions are expected for low class roads when lanes are widened.

Shoulder Width: For PDO crashes, increase in shoulder width up to $5 \mathrm{ft}$ is most effective at rural principal arterials (e.g., 29\% reduction for $5 \mathrm{ft}$ increase in shoulder width) but least effective at rural major collectors $(7 \%)$. Also, for fatal and injury crashes, increase in shoulder width is most effective at rural principal arterials (e.g., 37\% reduction in $5 \mathrm{ft}$ increase in shoulder width) but least effective for rural principal arterials $(17 \%)$. The result suggests that as the class of road increases, relatively higher percentages of crashes of all severities are saved by increases in shoulder width. This finding applies

for roads on the state highway system. For county roads, increase in shoulder width seems to have the same margin of effect as it does for rural principal arterials. 
Skid Resistance: For PDO crashes, increase in skid resistance width is more effective at rural major collectors (e.g., 9\% reduction for a 5 unit increase in friction number) but less effective at rural minor arterials $(6 \%)$. On the other hand, for fatal and injury crashes, increase in skid resistance is more effective at rural minor arterials (e.g., 15\% for a 5 unit increase in friction number) but least effective for rural major collectors $(8 \%)$. It can therefore be seen that as the class of road increases, relatively higher percentages of severe crashes and relatively lower percentage of non-severe crashes, are saved by increases in skid resistance.

Horizontal Alignment Improvement. For PDO crashes, increase in horizontal curve radius is most effective at rural minor arterials (e.g., 30\% reduction for 5-mile increase in radius) but least effective at rural major collectors (8\%). Also, for fatal and injury crashes, increase in lane width is most effective at rural minor arterials (e.g., $25 \%$ reduction for a 5 -mile increase in radius) but least effective for rural major collectors $(12 \%)$. Obviously, for the two extreme road classes (major collectors and principal arterials), relatively smaller percentages of all crash severity types are saved by improvements in horizontal alignment, compared to rural minor arterials. County roads seem to stand to benefit most from horizontal alignment improvements: a $77 \%$ reduction in crashes is expected for this countermeasure at such roads.

Vertical Alignment. For PDO crashes, increase in the quality of vertical alignment (decrease in grade) is most effective at rural major collectors (e.g., 90\% reduction for 6\% decrease in grade) but least effective in at rural principal arterials (48\%). Also, for fatal and injury crashes, increase in quality of vertical alignment is most effective at rural major collectors (e.g., $40 \%$ reduction for $6 \%$ decrease in grade) but least effective for rural principal arterials (34\%). This finding suggests that as the class of road decreases, relatively higher percentages of all crash severity types are saved by improvements in vertical alignment. 


\subsection{ESTIMATION OF COSTS OF SAFETY COUNTERMEASURES}

Table 7-5 presents the safety project costs. These were derived largely from available literature. Efforts are in progress to acquire data for such costs specific to the state of Indiana. For most of the cost elements shown below, it is not expected that Indiana data will be very different from those below.

Table 7-5: Safety Project Costs

\begin{tabular}{|c|c|c|c|c|}
\hline \multirow[b]{2}{*}{ Safety Improvement Project } & \multirow[b]{2}{*}{ Category } & \multicolumn{2}{|c|}{ Project Cost } & \multirow{2}{*}{$\begin{array}{l}\text { Reference } \\
\text { for } \\
\text { Project } \\
\text { Cost }\end{array}$} \\
\hline & & $\begin{array}{c}\text { Capital } \\
(\$ / \text { mile })\end{array}$ & Life (yr) & \\
\hline Groove roadway pavement & Pavement treatment & $\$ 29,442$ & 10 & (3) \\
\hline Chip Sealing to restore surface friction & Preventive Maintenance & $\$ 8,000$ & 8 & (4) \\
\hline Micro-surfacing to restore surface friction & Preventive Maintenance & $\$ 32,000$ & 8 & (4) \\
\hline Construct rumble strips in pavement & Construction/Reconstruction & $\$ 7,400$ & 7 & (1) \\
\hline Horizontal curve re-alignment (approx. value) & Construction/Reconstruction & $\$ 0.5 \mathrm{M}$ & 20 & (5) \\
\hline Improve pavement markings & Pavement Marking & $\$ 17,835$ & 20 & (3) \\
\hline Improve roadway lighting & Lighting & $\$ 674,578$ & 10 & (3) \\
\hline Install guardrail & Roadside Improvement & $\$ 184,401$ & 20 & (3), (5) \\
\hline Install paved shoulder & Construction/Reconstruction & $\$ 338,976$ & 20 & $(2)$ \\
\hline $\begin{array}{l}\text { Vertical curve realignment } \\
\text { (approx. value) }\end{array}$ & Construction/Reconstruction & $\$ 0.3 \mathrm{M}$ & 20 & (5) \\
\hline Widen Roadway lane by $1 \mathrm{ft}$ in both directions & Construction/Reconstruction & $\$ 52,800$ & 20 & $(2)$ \\
\hline Widen Shoulder by $1 \mathrm{ft}$ & $\begin{array}{l}\text { Construction/Reconstruction } \\
\text { including ROW Acquisition and } \\
\text { Clearing Obstacles in ROW }\end{array}$ & $\$ 61,242$ & 20 & $(2)$ \\
\hline
\end{tabular}

1. Harwood, D. W. [1993]. Use of Rumble Strips to Enhance Safety. NCHRP Synthesis 191, TRB, Washington, D.C.

2. Harwood, D. W., E. R. Kohlman Rabbani, K. R. Richard, H. W. McGee and G. L. Gittings. [2003]. Systemwide Impact of Safety and Traffic Operations Design Decisions of Resurfacing, Restoration, or Rehabilitation Projects. NCHRP Rep. 486, TRB, Washington, D.C.

3. FHWA. [2000]. Highway Economic Requirements System Technical Report, Federal Highway Administration, Washington, D.C.

4. Lamptey et al. [2004]. Life Cycle Cost Analysis for Pavement Design Procedures, Draft Final Report, FHWA/JTRP, Purdue University, W. Lafayette IN. 


\subsection{ESTIMATING THE COST-EFFECTIVENESS OF SAFETY ENHANCEMENTS}

Cost effectiveness may be defined as the ratio of the effectiveness (or benefits) in terms of number of crashes saved, to the cost of a safety countermeasure or set of countermeasures. The effectiveness can be expressed in terms of its crash reduction factor (the potential to reduce crashes from an existing level). As with any engineering system, improvements need to be evaluated also on the basis of both cost and effectiveness. A great amount of highway safety may be "bought" by implementing action, but the cost of that action may be prohibitive. While it is true that human life cannot be valued in monetary terms from an ethical viewpoint, the practical reality is that agencies operate within a constrained budget and cannot afford to implement the most effective countermeasures that are also the most expensive. Agencies are typically interested in deciding at which sections to "invest" a limited budget such the returns (crash reduction) would be maximized. Furthermore, agencies seek to implement temporary palliative measures at deficient sections until such a time when reconstruction or large scale physical improvements can be funded. In such cases, it is useful to determine the cost-effectiveness of safety countermeasures. In the present study, cost effectiveness analysis was carried out for the following categories of roads:

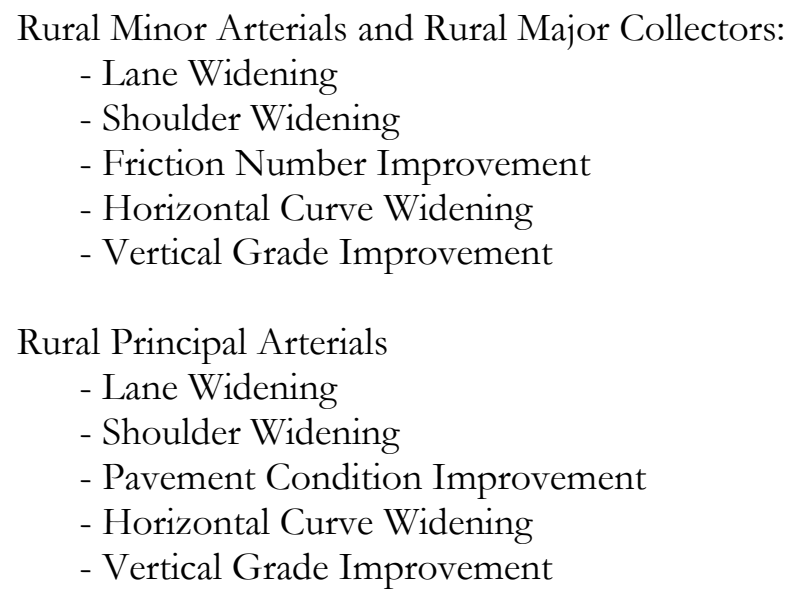

Cost-effectiveness analysis for county roads was not carried out due to lack of cost data at such roads. However, the methodology presented in this chapter offers an opportunity to carry out the analysis for such roads at a future date when cost data become available. 


\subsubsection{Cost-effectiveness of Safety Enhancements at Rural Major Collectors}

\subsubsection{Cost-effectiveness by Extent of Countermeasure}

Figure 7-4 shows that for injury and PDO crashes, increasing the lane width of Indiana's rural major collectors is associated with increasing cost-effectiveness up to a $2 \mathrm{ft}$ increase in lane-width, and a decreasing cost-effectiveness thereafter. For fatal crashes, increases in lane-width are consistently associated with decreased cost-effectiveness. Beyond $3 \mathrm{ft}$ of additional lane-width, the decrease in cost effectiveness for all crash severity types seems to flatten out. The average existing lane width at rural major collectors is approximately $10.5 \mathrm{ft}$. an increase. For shoulder width increases, the results seemed to be equivocal: for PDO crashes, shoulder widening cost-effectiveness seems to increase up to $2 \mathrm{ft}$ of widening, and drops gently thereafter. For the other crash severity types, very gentle or no drops in cost-effectiveness is observed for increasing shoulder width. With regard to pavement surface friction, surface treatments such as chip sealing micro-surfacing are known to offer significant benefits directly in terms of skid resistance. The previous section shows that the indirect benefit in terms of crash reduction is very significant. Figure 7-4 shows that chip sealing is almost twice as costeffective (but less effective) compared to micro-surfacing in addressing friction problems at rural major collectors. The effectiveness trends for increasing horizontal curve radius were mixed, but generally followed a trend of decreasing cost-effectiveness with increasing enhancements to horizontal curve radius.

The generally decreasing cost-effectiveness of the above treatments with increasing effort seems to reflect the fact the increasing efforts leads to disproportionate increases in costs. For example, in order to widen a lane or shoulder, or to reduce horizontal curve radius, a significant amount of money is needed to remove topsoil, fill the existing drain with approved material, fill the relevant portion of low-lying surrounding terrain to finished road level, and laying/compacting of pavement material. If more width is needed, the agency moves from an already acquired right-of-way into abutting land and therefore additional money is needed to acquire additional right-of-way, and to relocate or demolish existing fence wall and other structures on the acquired right-of-way. Such increases in costs as the extent of road or shoulder widening, is obviously enough to offset any economies of scale arising from increased work output, and may lead to decreased cost-effectiveness of such increasing efforts as reflected in Figure 7-4. In the case of friction number enhancement, large increases in friction number does not involve additional purchases of ROW, but involves a surface treatment (micro-surfacing) that is over 4 times as costly as the less expensive option (chip sealing) but is only 2-3 times more effective in improving pavement condition (Labi, 2001). For increasing quality of vertical alignment (decreasing vertical grade), a increasing the effort of 
improvement (through further reductions in vertical grade) is associated with increasing costeffectiveness. like the case for horizontal curve improvements, vertical alignment improvements is achieved through capital intensive work such as excavation and filling. However, unlike the case for horizontal curves, additional right-of-way is typically not required for vertical alignment enhancements, as such increasing enhancement efforts do not translate to significant increases in costs. It is therefore not surprising to see that increasing enhancements in vertical alignment is associated with increasing cost-effectiveness. This relationship is more pronounced for PDO crashes and less pronounced for fatal and injury crashes.

\subsubsection{Cost-effectiveness by Type of Countermeasure}

Just as was stated in the previous section for effectiveness, it may not be very meaningful to compare the cost-effectiveness across different countermeasures except in the following situations: (i) the countermeasures are viable mutually exclusive alternative actions that remedy a given safety problem at an existing road, or (ii) an existing road suffers from multiple types of safety problems thus many different countermeasures may be carried out but funds are available to carry out only relatively few of these improvements.

Figure 7-4 suggests that improvement in surface skid resistance (friction) is most costeffective in terms of the percentage of crashes reduced (2-5\% of existing crashes saved per $\$ 10,000$ expended in that effort). Vertical grade enhancement yield $0.1-0.3 \%$ crashes saved per $\$ 10,000$ expenditure, while horizontal realignment yield only $0.03-0.06 \%$ crashes saved per $\$ 10,000$. As such, for a winding road section suffering from both vertical and horizontal alignment deficiencies, higher returns for the dollar may generally be achieved by investing the available money on the vertical alignment. Another interesting case is that of lane widening and shoulder widening. Faced with a narrow road, agencies are typically faced with a dilemma of whether to increase the lane-width or to increase the shoulder width. High costs are encountered in lane extensions (due to high cost of pavement surfacing material), but the cost data suggests that such costs are outweighed by the extra right-of-way acquisition costs that are often associated with shoulder extensions. Besides, a unit increase in shoulder width, as seen from the previous section of this chapter, has a lower effectiveness (lower crash reduction) than a unit increase in lane width. As such, shoulder extensions are less costeffective compared to lane additions. It is therefore seen from Figure 7-4 that agencies generally stand to reap, on the average, three times as many returns per dollar if they choose to widen an existing lane rather than adding a shoulder. It must be noted that these are average conditions only. For a road 
with lanes that are already very wide and no shoulder, it is generally more worthwhile to provide a shoulder rather than extend the existing lane.

\subsubsection{Cost-effectiveness by Crash Severity Type}

The results shown in Figure 7-4 suggest that for lane width treatments, the levels of cost-effectiveness are relatively higher for fatal and injury crashes compared to PDO crashes. However, the change in cost-effectiveness per unit increase in lane width seems fairly similar across all crash types. A similar observation is made for shoulder widening treatments and horizontal curve enhancements. For friction number improvement, the level and sensitivity of the treatment seemed to be uniform across all crash severity types. For vertical grade improvements however, it is seen that the treatment is most effective for PDO crashes and less cost effective for fatal and injury crashes. Also, the sensitivity of cost-effectiveness for a unit change in vertical enhancement effort, is relatively high for PDO crashes compared to fatal and injury crashes. 

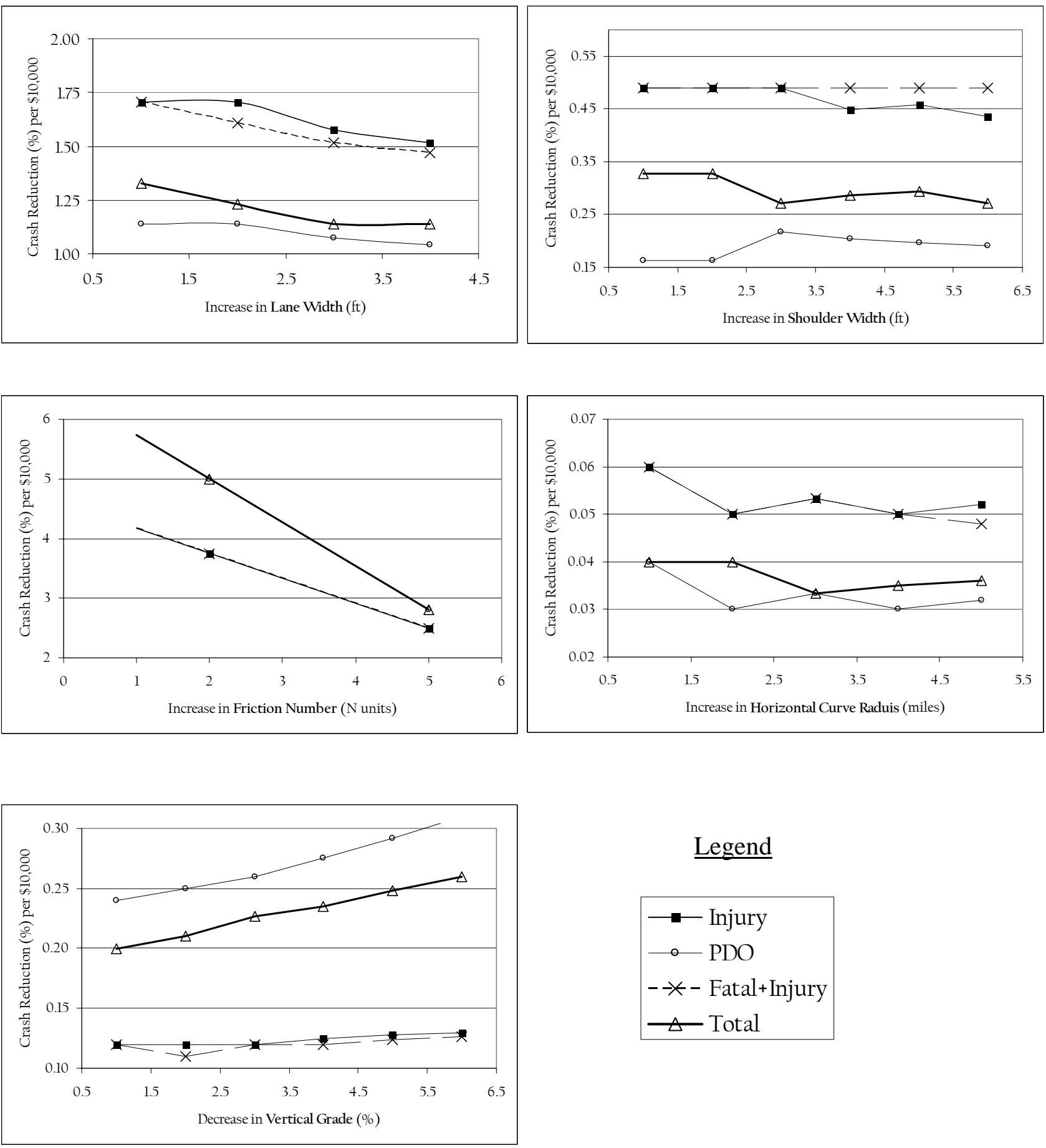

\section{$\underline{\text { Legend }}$}

\begin{tabular}{l}
$\longrightarrow-$ Injury \\
- PDO \\
$--x-$ Fatal+Injury \\
$\longrightarrow$ Total \\
\hline
\end{tabular}

Figure 7-4: Cost-effectiveness by Improvement Type and Crash Type, Rural Major Collectors

\subsubsection{Cost-effectiveness of Safety Enhancements at Rural Minor Arterials}




\subsubsection{Cost-effectiveness by Extent of Countermeasure}

For rural minor arterials, the general cost-effectiveness trends were found to be similar to those of rural major collectors. As seen in Figure 7-5, increasing the lane width of Indiana's rural minor arterials is associated with generally decreasing cost effectiveness up to a $2 \mathrm{ft}$ increase in lane-width, and a rather sharply decreasing cost-effectiveness thereafter, but does not flatten out as is the case for rural major collectors. The average existing lane width at rural major collectors is $11-12 \mathrm{ft}$. It is also seen that cost-effectiveness gently decreases with increasing extension in shoulder width. The costeffectiveness of pavement surface friction improvements decreases with increasing effort - this trend varies by crash severity type but tends to converge with increasing effort. For all crash severity types, increasing horizontal radius is associated with gently decreasing cost effectiveness, while increases in vertical alignment quality is associated with increased cost-effectiveness, for reasons similar to those offered in the case of rural major collectors.

\subsubsection{Cost-effectiveness by Type of Countermeasure}

Similar to the case for rural major collectors, improvement in surface skid resistance (friction) per unit cost of efforts seems to be highest compared to other safety treatments, with $1.5-9 \%$ reduction in crashes per $\$ 10,000$. For vertical grade enhancement, with there is a $0.16-0.26 \%$ reduction in crashes per $\$ 10,000$ expenditure. Horizontal realignment, on the other hand, has only $0.1-0.15 \%$ reduction in crashes per $\$ 10,000$. Also similar to the case for rural major collectors, lane widening at rural minor arterials seem to be more 3-6 times more cost-effective than shoulder widening.

\subsubsection{Cost-effectiveness by Crash Severity Type}

As seen in Figure 7-5, lane widening is associated with relatively little differences in cost-effectiveness across crash severity types. In contrast, the cost-effectiveness levels for the various crash types are rather significantly different across the various crash types for shoulder widening, friction enhancements, and horizontal and vertical alignment treatments. For surface friction improvements, cost-effectiveness levels are relatively higher for fatal and injury crashes compared to PDO crashes. 

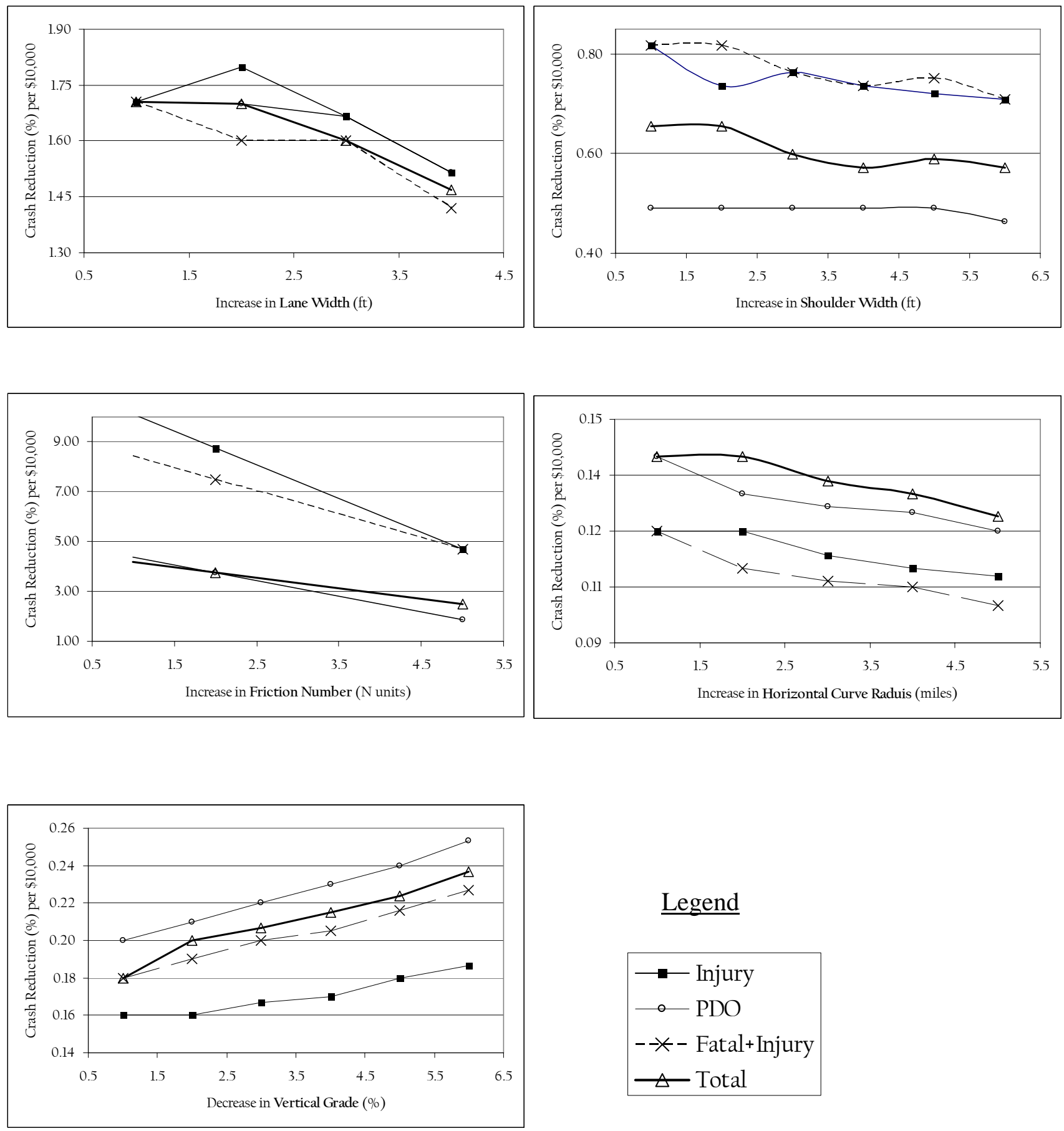

Figure 7-5: Cost-effectiveness by Improvement Type and Crash Type, Rural Minor Arterials 


\subsubsection{Cost-effectiveness of Safety Enhancements at Rural Major Principal Arterials}

\subsubsection{Cost-effectiveness by Extent of Countermeasure}

The patterns of cost-effectiveness were generally similar to those of rural major collectors and minor arterials. As Figure 7-6 shows, increasing the lane width of Indiana's rural minor arterials is associated with gradual decreases in cost-effectiveness. The average existing lane width at rural major collectors exceeds $12 \mathrm{ft}$. It is also seen that cost-effectiveness gently decreases with increasing extension in shoulder width up to $5 \mathrm{ft}$ extension, and decreases sharply thereafter. For pavement surface friction improvements, cost-effectiveness decreases with increasing effort, varying by crash severity type but converging with increasing effort. Increasing horizontal alignment improvement effort as represented by increasing radius is associated with gently decreasing cost effectiveness, for all crash severity types. On the other hand, an increase in vertical alignment quality is associated with increased cost-effectiveness, for reasons similar to those offered in the case of rural major collectors and principal arterials.

\subsubsection{Cost-effectiveness by Type of Countermeasure}

Pavement condition improvement has the highest level of cost-effectiveness in terms of $\%$ crash reduction per dollar. For rural principal arterials with poor pavement surface quality, as much as $22 \%$ reduction in fatal and injury crashes and 8\% reduction in PDO crashes can be earned for every $\$ 10,000$ invested in improvements in pavement condition. For vertical grade enhancement, with there is a $0.1-0.16 \%$ reduction in crashes per $\$ 10,000$ expenditure, depending on crash severity type. Horizontal realignment yields $0.06-0.1 \%$ reduction in crashes per $\$ 10,000$. Also similar to the case for rural major collectors and minor arterials, lane widening at rural principal arterials is significantly more cost-effective than shoulder widening.

\subsubsection{Cost-effectiveness by Crash Severity Type}

For rural principal arterials, lane widening is associated with significant differences in costeffectiveness across crash severity types. Also, the cost-effectiveness levels for the other crash types are rather significantly different for shoulder widening, friction enhancements, and horizontal and vertical alignment treatments. For shoulder widening and pavement condition improvements, costeffectiveness levels are relatively higher for fatal and injury crashes compared to PDO crashes. For horizontal and vertical alignment improvements, cost-effectiveness levels are higher for PDO crashes compared to fatal and injury crashes. 

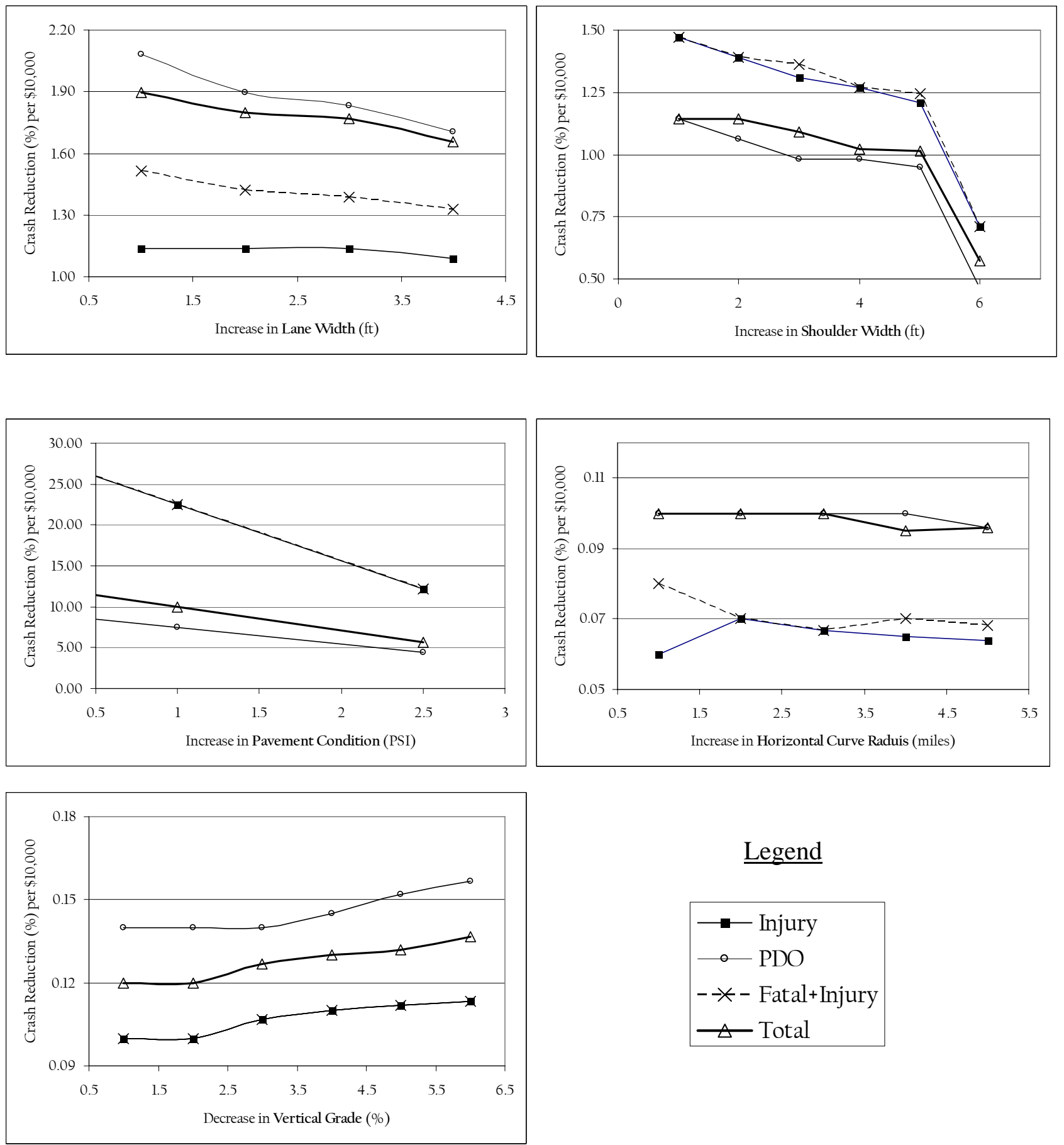

\section{$\underline{\text { Legend }}$}

$\longrightarrow-$ Injury
- PDO
$--x-$ Fatal+Injury
$\triangle-$ Total

Figure 7-6: Cost-effectiveness by Improvement Type and Crash Type, Rural Principal Arterials 


\subsubsection{Cost-effectiveness Findings Across Road Classes}

While the trends in cost-effectiveness relative to treatment effort were generally similar across the three classes of state highways, there were a few differences in cost-effectiveness levels across them. On the basis of PDO crashes, increases in lane width seem to be more cost-effective at rural principal arterials (approximately 1.9\% crash reduction per $\$ 10,000$ ) than the other road classes. On the basis of fatal and injury crashes, there seemed to be generally similar average cost-effectiveness levels across the road classes. Also, increases in shoulder width up to $5 \mathrm{ft}$ are most cost-effective at rural principal arterials and least cost-effective at rural major collectors. It is also seen that on the basis of fatal and injury crash reduction per dollar, friction improvement has a greater cost-effectiveness at rural minor arterials compared to rural major collectors. However, on the basis of PDO crash reduction per dollar, friction improvement has a lower cost-effectiveness at rural minor arterials compared to rural major collectors. Horizontal curve improvements seem to have the highest costeffectiveness at rural minor arterials and the least cost-effectiveness at rural collectors. Also, costeffectiveness of horizontal curve improvements at rural minor arterials appear to be more sensitive to increases in the extent of this treatment compared to rural major collectors or principal arterials. Furthermore, at rural major collectors, horizontal curve improvement has a greater cost-effectiveness in terms of fatal and injury crashes compared to PDO crashes, while the reverse is true for rural minor arterials and principal arterials. Vertical alignment improvement cost-effectiveness levels were similar for rural major collectors and minor arterials and were higher than those for rural principal arterials. It is interesting to note that for rural major collectors and principal arterials, fatal and injury crashes have similar levels of vertical alignment improvement cost effectiveness while for rural minor arterials, this treatment is more cost-effective for fatal crashes than it is for injury crashes.

\subsubsection{Incremental Crash Reductions of Selected Countermeasures for Existing Levels of Selected Geometric Features}

The crash reduction factors developed in this section represent percentage reductions from existing crash levels. For a given crash reduction factor, a higher level of existing crashes would yield a greater number of crashes reduced. The greater the effort made for a given countermeasure, the greater the reduction in crashes. However, as seen in Tables 7-1 to 7-3, the relationship may not be linear - an increase in effort at a lower level of the countermeasure may have a different crash reduction compared to the same increase at a higher level of the countermeasure. For example, a 0 to $4 \mathrm{ft}$. increase in shoulder width may not have the same impact (crash reduction) as a 6 to $10 \mathrm{ft}$. increase. Even when the initial values of the geometric feature are the same for two sections, one may accrue a 
higher crash reduction because it may have a different level of another geometric feature besides that under consideration. For example, in increasing the shoulder width from 2 to $6 \mathrm{ft}$., the section with narrow lanes may see a greater reduction in crashes compared to that with already wide lanes. It is possible for an analyst to develop any of these combinatorial relationships given the crash prediction equations and crash reductions factors made available in the present study. To illustrate how this could be done, two cases are herein discussed:

1. Effect of lane widening at two typical rural two-lane sections - one section has no shoulder and the other has a $6 \mathrm{ft}$. shoulder. Other conditions are as follows: Section Length - 15 miles, Section AADT - 10,000, Friction Number - 2, Avg. Radius of Horizontal curves - $200 \mathrm{ft}$., Avg. Grade of Vertical Curves - 2\%.

The differences in expected crash frequencies corresponding to each case and condition is illustrated as Figure 7-7. It is seen that irrespective of shoulder width, increasing the lane width generally decreases crash frequency. Also, for a given existing lane width, greater extensions in the lane width yields lower crashes (greater reductions in crash frequency). Then, for a given width extension, the narrower lanes seem to yield slightly greater reductions in crash frequency compared to wider lanes. Most importantly, there is a clear difference in crash frequencies (and reductions thereof) for widened lanes without a shoulder and with a wide shoulder.

2. Effect of shoulder widening at two typical rural two-lane sections - one section has a narrow lane $(7 \mathrm{ft}$.$) and the other has a wide lane (10 \mathrm{ft}$.) Other Conditions are as follows: Section Length - 15 miles, Section AADT - 10,000, Friction Number - 2, Avg. Radius of Horizontal curves $-200 \mathrm{ft} .$, Avg. Grade of Vertical Curves - 2\%.

This is illustrated as Figure 7-8. It is seen for both narrow- and wide-lane sections, increasing the shoulder width generally leads to a decrease in crash frequency. Furthermore, for a given existing shoulder width, greater extensions in the shoulder width yields lower crashes (greater reductions in crash frequency). Then, for any given extent of shoulder width extension, the section with no shoulder seem to yield an almost imperceptible but greater reduction in crash frequency compared to the section with a shoulder. Another interesting result is the clear differences in crash frequencies (and reductions thereof) for shoulder widening at narrowlane sections compared to the wide-lane sections. 


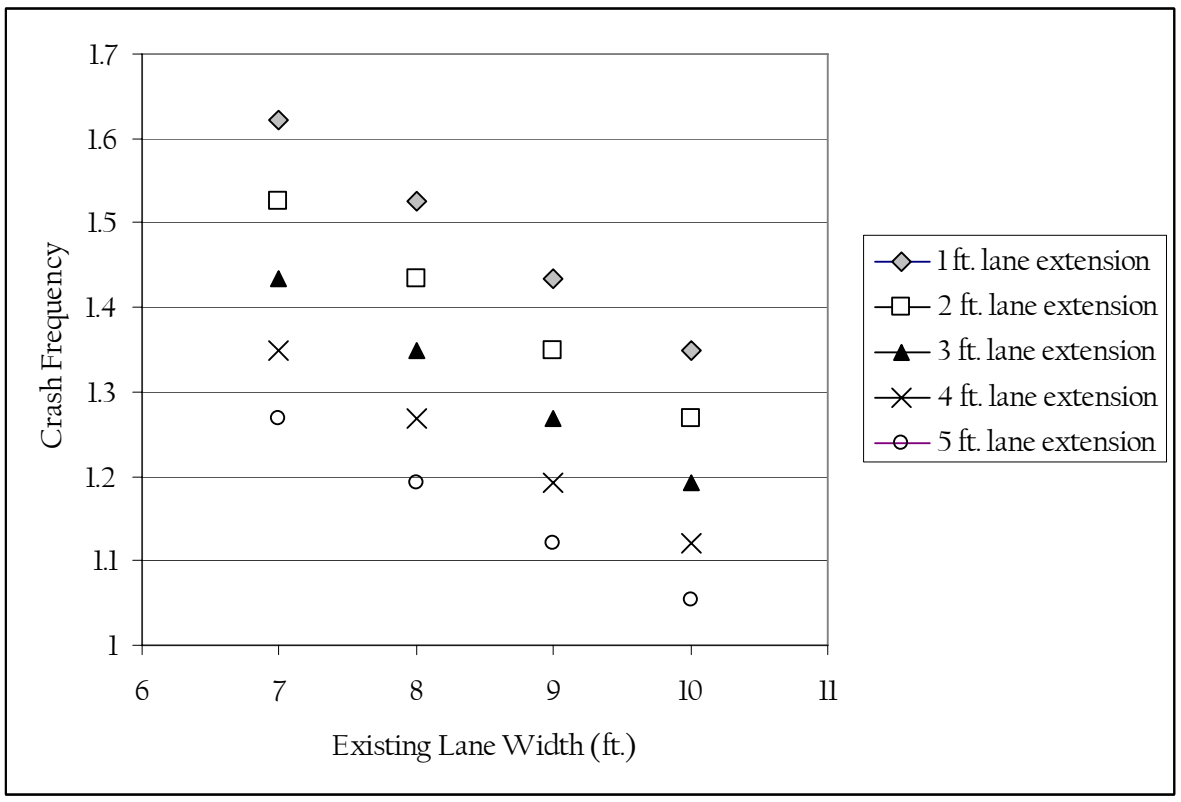

(a) No Shoulders

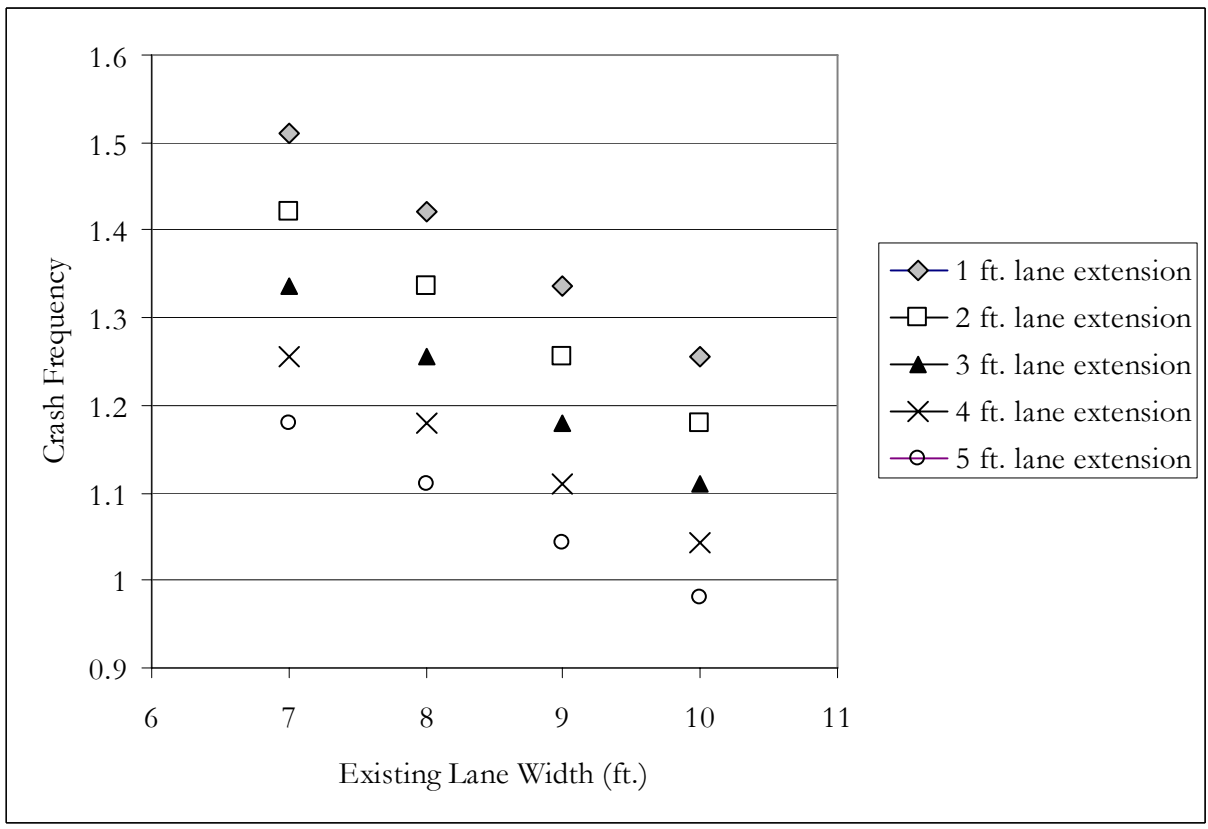

(b) $6 \mathrm{ft}$. Shoulders

Figure 7-7: Effect of Lane Widening at Typical Rural Two-Lane Sections

Other Conditions: Section Length - 15 miles, Section AADT - 10,000, Friction Number - 2, Avg. Radius of Horizontal curves - $200 \mathrm{ft}$., Avg. Grade of Vertical Curves - 2\% 


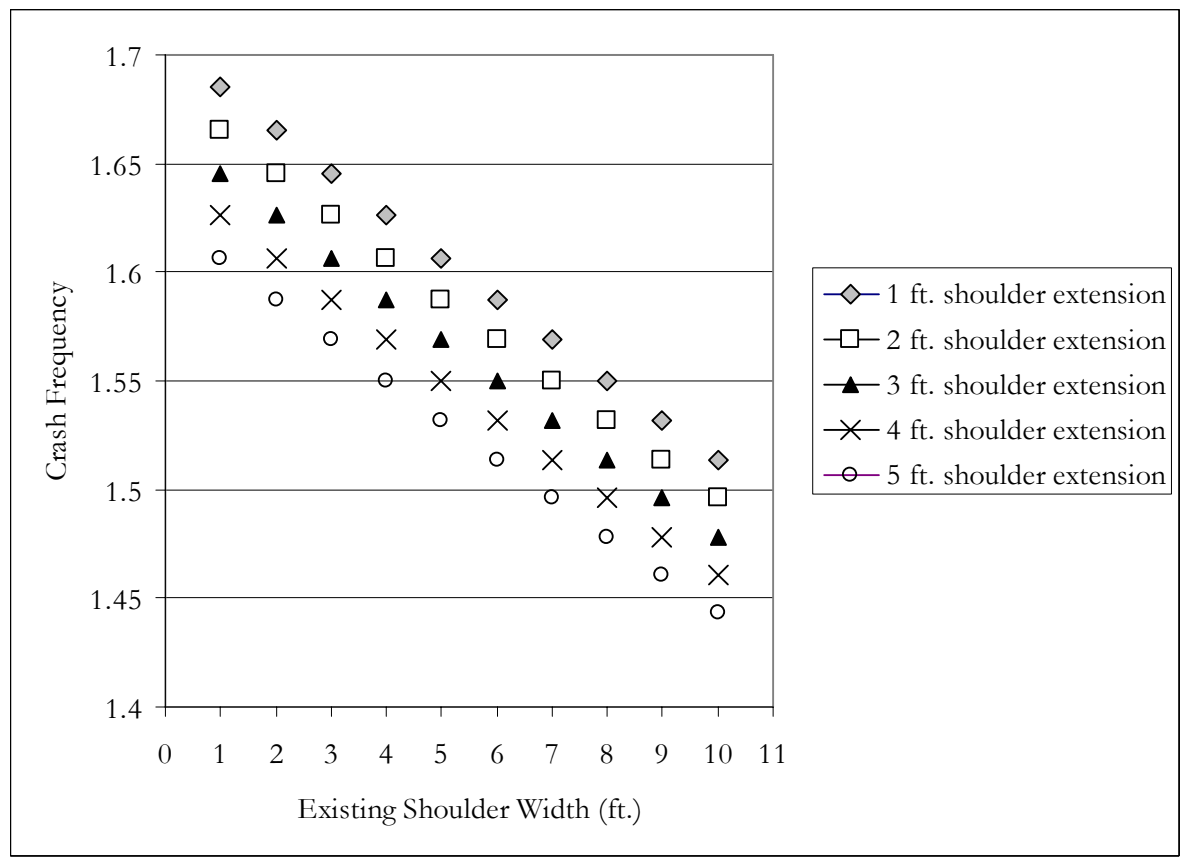

(a) Narrow (7 ft.) Lanes

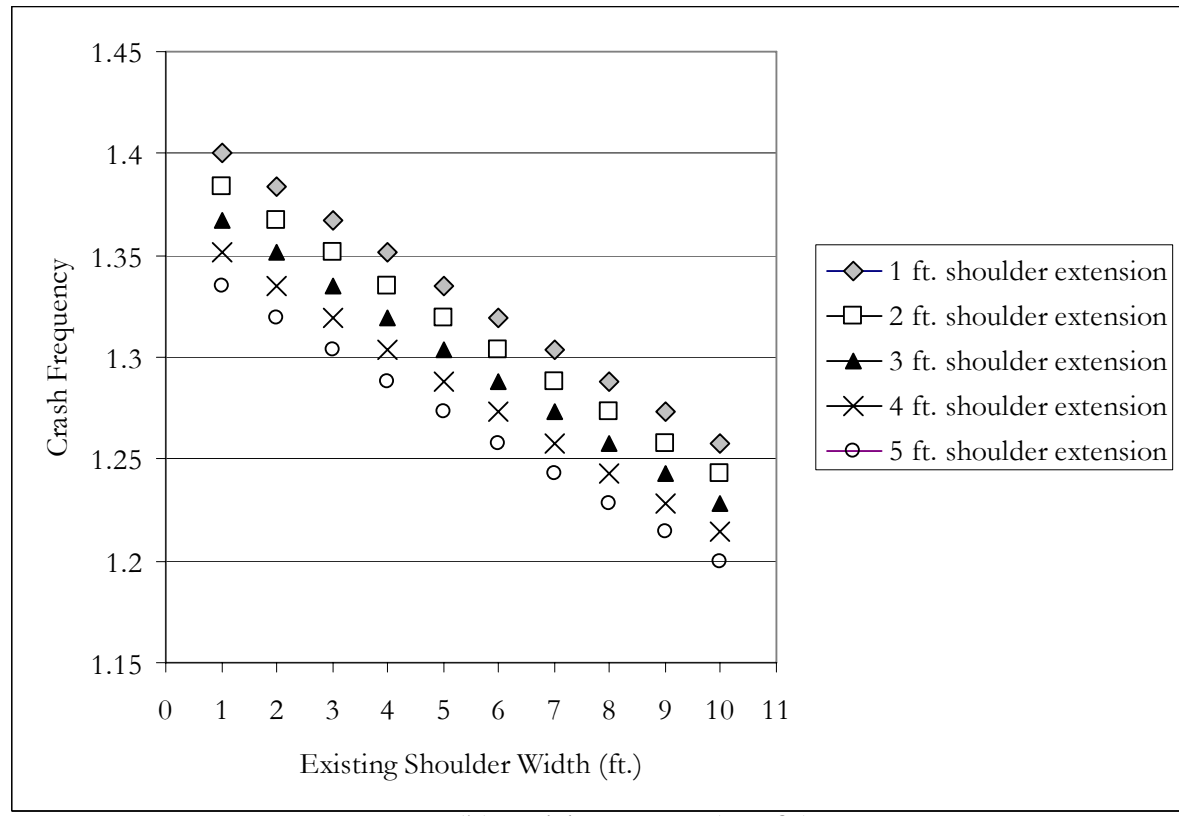

(b) Wide Lanes (10 ft.)

Figure 7-8: Effect of Shoulder Widening at Typical Rural Two-Lane Sections

Other Conditions: Section Length - 15 miles, Section AADT - 10,000, Friction Number - 2, Avg. Radius of Horizontal curves - $200 \mathrm{ft.}$. Avg. Grade of Vertical Curves - 2\% 


\section{CHAPTER 8 NETWORK-LEVEL SAFETY NEEDS ASSESSMENTS}

\subsection{INTRODUCTION}

The previous chapter focuses on how INDOT and LTAP can estimate the effectiveness and costeffectiveness of safety improvements at specific road sections. However, in certain cases, these agencies may be interested in identifying which sections in their network are deficient, determining the most cost-effective countermeasure at each section, and prioritizing all such needed safety improvements for the entire network over an analysis period and within a given budgetary constraint. The present chapter addresses this issue for Indiana's two-lane rural state highway network but ca easily be extended for county roads after requisite data are available for the latter. For each year within the analysis period, the chapter assesses the physical needs (where are improvements needed and what kind?) and monetary needs (how much is needed to address identified physical needs?).

The chapter begins with a list of the various design standards for rural two-lane roads and then identifies all sections on the state's rural two-lane system that have at least one deficiency. Deficiencies for each section on the state's rural two-lane highway system, as well as general summaries of such deficiencies by deficiency type, are presented. The chapter also provides details and a summary of capital requirement for addressing safety at the state's rural two-lane state highways. Finally, using three alternative budgetary ceilings for rural two-lane state highways, the study proceeds to identify where such funds could be best used so that overall cost-effectiveness is maximized.

\subsection{EXISTING INDOT DESIGN STANDARDS FOR RURAL TWO-LANE ROADS}

For each rural two-lane state highway section, the cross-sectional elements were compared with the design standards specified in the Indiana Design Manual. Any section that fell short of the standards was flagged as a deficient section and appropriate improvement projects assigned to it. A summary of the design standards for rural two roads used in the analysis is shown in Table 8-1. The table shows that the design standards for rural principal arterials are same as those for rural minor arterials, and are more stringent than the design standards for rural major collectors. For example, the minimum lane width for a rural two-lane arterial is $12 \mathrm{ft}$, while that for a major collector is $10 \mathrm{ft}$. Also, the 
minimum shoulder width for an arterial is $6 \mathrm{ft}$, while that for a collector is $4 \mathrm{ft}$. Access control standards are same across functional classes. The minimum horizontal curve radius for arterials is 837 $\mathrm{ft}$, while that for collectors is $443 \mathrm{ft}$. In highway geometric design, adequate but not excessive vertical grade is needed to ensure drainage while maintaining safe vehicle operation. For arterials, vertical alignment grade should be $0.5-5 \%$, while that for collectors is $0.5-8 \%$. In other words, sharper horizontal curves and steeper vertical grades seem to be tolerated to a greater extent at rural collectors compared to the other two highway classes. Furthermore, in each functional class, design standards vary by traffic volume. Higher traffic volumes, all else being equal, translate to higher design standards.

Table 8-1 Indiana Design Standards for Rural Two-Lane State Highways

\begin{tabular}{|c|c|cc|}
\hline \multirow{2}{*}{$\begin{array}{c}\text { Cross } \\
\text { Sectional Element }\end{array}$} & \multirow{2}{*}{$\begin{array}{c}\text { FADT } \\
\text { Range }\end{array}$} & Two-Lane Arterials & Major Collectors \\
\cline { 3 - 4 } & $<450$ & 12 & 10 \\
Lane Width (ft) & $450-2000$ & 12 & 11 \\
& $>2000$ & 12 & 12 \\
\hline \hline \multirow{3}{*}{ Right Shoulder Width (ft) } & $<450$ & 6 & 6 \\
& $450-2000$ & 6 & 6 \\
\hline \multirow{3}{*}{ Access Control } & $>2000$ & 6 & 2 \\
& $<450$ & 2 & 2 \\
\hline \multirow{2}{*}{ Horizontal Curve Radius (ft) } & $450-2000$ & 2 & 443 \\
& $>2000$ & 2 & 640 \\
& Low speed & 837 & 837 \\
\hline \multirow{2}{*}{ Vertical Curve Grade (\%) } & High Speed & 1116 & 0.5 \\
& Minimum & 1444 & 7 \\
\hline INDOT Design Manual INDOT, 20007 & Desired & 0.5 & 8 \\
\hline
\end{tabular}

\subsection{NEEDS ASSESSMENT FOR INDIANA'S RURAL TWO-LANE STATE HIGHWAYS}

Using the needs assessment methodology explained in Chapter 3, the present study carried out an assessment of physical and monetary needs associated with geometric deficiencies at the state's rural two-lane highways. Table 8-2 presents a summary of the deficiencies obtained from the analysis, and shows that the most common deficiencies at Indiana's rural two-lane state highway system are inadequate shoulder and lane widths (such deficiencies represent approximately $60 \%$ and 36\%, respectively, of all deficiencies). This could be attributed to the fact that the rural two-lane state highways were originally designed to relatively low design standards due to their relatively low traffic volume compared to other functional classes on the state highway system. The analyses in Chapters 6 
and 7 show that such inferior design standards are associated with (and obviously, largely responsible for) the relatively high crash rates on such roads.

Table 8-2 Summary of Identified Deficiencies on Rural Two Lane Roads

\begin{tabular}{|l|c|c|}
\hline Deficiency & $\begin{array}{c}\text { Number of Deficient } \\
\text { Sections }\end{array}$ & $\begin{array}{c}\text { Length of miles for } \\
\text { Improvements (Miles) }\end{array}$ \\
\hline $\begin{array}{l}\text { Road section has at least one “poor" } \\
\text { horizontal curve (radius less than 1444 ft) }\end{array}$ & 91 & 19.97 \\
\hline $\begin{array}{l}\text { Road section has at least one "very poor" } \\
\text { horizontal curve (radius less than 837 ft) }\end{array}$ & 151 & 33.65 \\
\hline Road section has no shoulder & 421 & 426.14 \\
\hline $\begin{array}{l}\text { Road section has average narrow lanes (width } \\
\text { less than 12 ft) }\end{array}$ & 840 & $3,736.22$ \\
\hline $\begin{array}{l}\text { Road section has average narrow outside } \\
\text { shoulder (width less than 4 ft) }\end{array}$ & 7 & 14.93 \\
\hline $\begin{array}{l}\text { Road section has outside shoulder less than 6 } \\
\mathrm{ft}\end{array}$ & 1,308 & $6,036.24$ \\
\hline Vertical grade greater than 5\% & 22 & 23.61 \\
\hline \multicolumn{2}{|c|}{18} & 27.63 \\
\hline
\end{tabular}

Based on data from Indiana Safety Management System database [Lamptey et al., 2003].

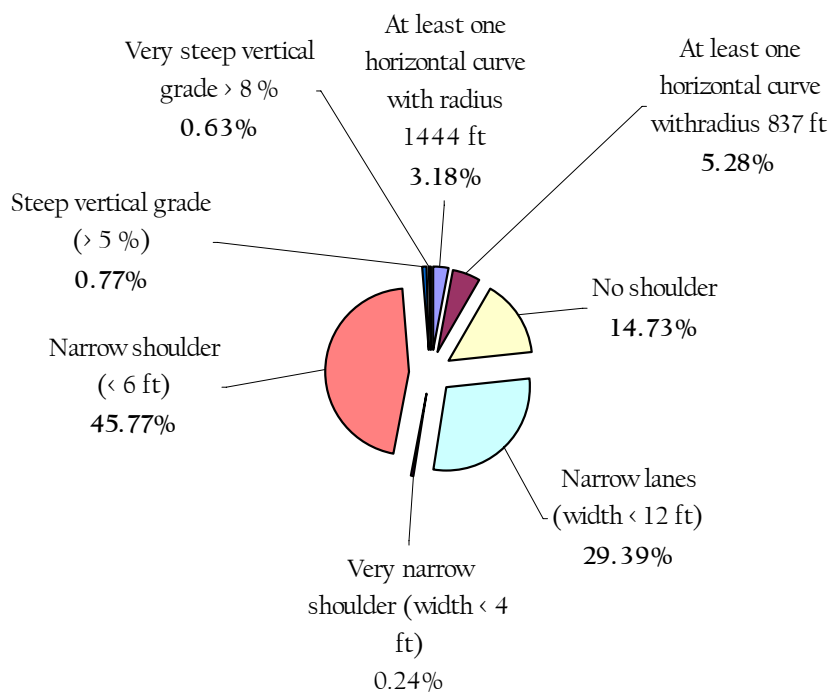

$\%$ System Deficiency by Nr. of Sections

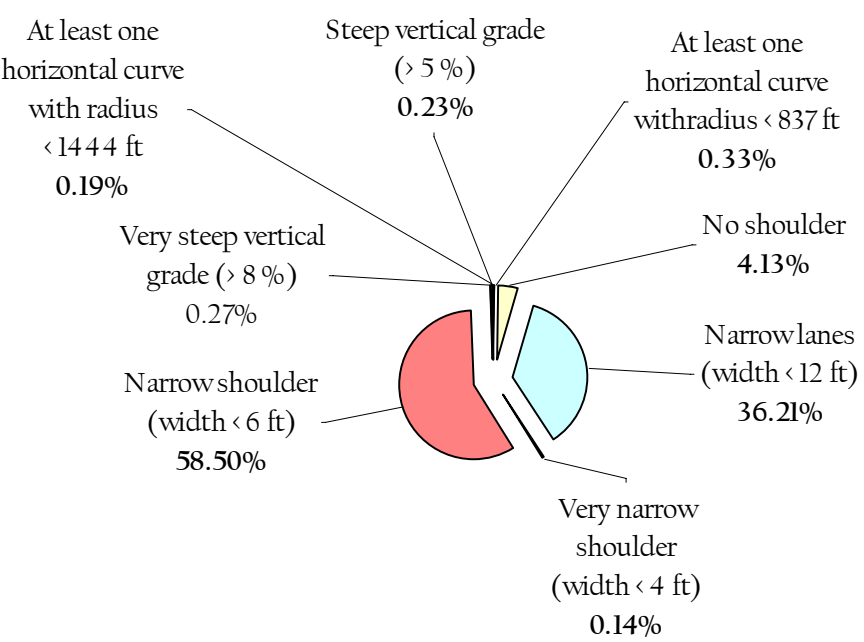

$\%$ System Deficiency by Mileage

Figure 8-1: Summary of Identified Deficiencies on Rural Two Lane Sate Highways in Indiana 
An assessment of the monetary needs to address the physical problems on the state's rural two-lane system can be carried out on the basis of sections that are identified as geometrically deficient, or sections that are both geometrically deficient and hazardous.

Needs assessment can be carried out on the basis of deficient sections or both deficient and hazardous sections. Also, needs assessments can be carried out on the basis of economic costs or comprehensive crash costs. Furthermore, such assessments may be unconstrained (that is, on the assumption of unlimited funding) or may be prioritized due to budgetary constraints. For the entire rural two-lane state highway network in Indiana, safety enhancement needs were assessed in the present study on the basis of urgency criterion (deficient or deficient+hazardous), cost types (economic vs. comprehensive). Also prioritization was carried out for various budgetary ceilings. Specifically, the following scenarios were used to assess the safety needs of the rural two-lane road network as shown in Table 8-3. The results for each scenario are hereby presented and discussed.

Table 8-3: Needs Assessment and Prioritization Scenarios

\begin{tabular}{|l|l|c|c|c|}
\hline \multicolumn{2}{|l|}{ Description of Scenario } & $\begin{array}{l}\text { Budgetary } \\
\text { Limit }\end{array}$ & $\begin{array}{l}\text { Economic } \\
\text { Crash Costs }\end{array}$ & $\begin{array}{l}\text { Comprehensive } \\
\text { Crash Costs }\end{array}$ \\
\hline $\begin{array}{l}\text { Sections that are only } \\
\text { Deficient but not Hazardous }\end{array}$ & Unlimited Budget & - & \multicolumn{2}{|c|}{ Scenario 1 } \\
\hline \multirow{3}{*}{$\begin{array}{l}\text { Sections that are both } \\
\text { Deficient and Hazardous }\end{array}$} & Unlimited Budget & - & \multicolumn{2}{|c|}{ Scenario 2 } \\
\cline { 2 - 6 } & $\begin{array}{l}\text { Limited Budget } \\
\text { (Prioritization) }\end{array}$ & $\$ 1$ million & Scenario 3 & Scenario 4 \\
\cline { 2 - 6 } & $\begin{array}{l}\text { Limited Budget } \\
\text { Prioritization) }\end{array}$ & $\$ 2$ million & Scenario 5 & Scenario 6 \\
\hline
\end{tabular}

\subsubsection{Needs Assessment for Sections that are Deficient but not Hazardous (Scenario 1)}

From the monetary needs assessment carried out using INDOT's Safety Management System Software package, it was found that the cost of improvements required to upgrade the deficient sections to design standards is estimated at $\mathbf{\$ 5 3 0}$ million, in Year 2000 constant dollars. It is possible that by the time of reporting, a few more sections have been upgraded to eliminate such deficiencies. Table 8-4 shows a summary of the total required capital (monetary needed) by project type, the overall estimated monetary benefits, and the expected total reduction in crashes if the identified deficiencies are addressed.

Table 8-4 Summary of Capital Requirement and Benefits by Project Type, Scenario 1 
Needs Assessment (i.e., No Budgetary Limit) Basis of Selection: All Identified Deficient Sections, no Regard to their Hazardousness)

\begin{tabular}{|c|c|c|c|c|c|}
\hline \multirow[b]{2}{*}{ Improvement Project } & \multirow{2}{*}{$\begin{array}{l}\text { Capital } \\
\text { Required }^{2}\end{array}$} & \multirow{2}{*}{$\begin{array}{l}\text { Estimated } \\
\text { Benefits }^{2}\end{array}$} & \multicolumn{3}{|c|}{ Crash Reduction $^{1}$} \\
\hline & & & $\begin{array}{l}\text { Fatal/ } \\
\text { Injury }\end{array}$ & PDO & Total \\
\hline Horizontal Curve Re-alignment Projects & $\$ 2,946,000$ & $\$ 1,815,241$ & 0 & 0 & 0 \\
\hline Shoulder Paving Projects & $\$ 40,500,852$ & $\$ 51,653,960$ & 21 & 96 & 117 \\
\hline Vertical Curve Re-alignment Projects & $\$ 2,469,000$ & $\$ 1,627,280$ & 0 & 4 & 4 \\
\hline $\begin{array}{l}\text { Lane Widening Projects }(1 \mathrm{ft} \text { in each } \\
\text { direction) }\end{array}$ & $\$ 190,186,656$ & $\$ 441,463,256$ & 331 & 621 & 952 \\
\hline $\begin{array}{l}\text { Shoulder Widening Projects }(2 \mathrm{ft} \text { in each } \\
\text { direction) }\end{array}$ & $\$ 182,922,190$ & $\$ 198,312,424$ & 122 & 453 & 575 \\
\hline $\begin{array}{l}\text { Shoulder Widening Projects ( } 4 \mathrm{ft} \text { in each } \\
\text { direction) }\end{array}$ & $\$ 110,689,936$ & $\$ 116,843,654$ & 78 & 275 & 353 \\
\hline TOTAL & $\$ 529,714,635$ & $\$ 811,715,815$ & 552 & 1449 & 2001 \\
\hline
\end{tabular}

An analysis based only on existing geometric deficiencies such as that shown above and in Appendix A-1 is often desirable due to its simplicity. However, (i) a deficient section is not necessarily hazardous, and it may not be worthwhile to carry out safety investments at sections that are deficient but not hazardous, (ii) the high cost of upgrading all deficient sections on Indiana's rural two-lane sections (which is the ideal but impractical solution to all engineering-related safety problems at these roads) is likely to be beyond INDOT's financial capabilities. As such, there is a need to further filter the deficient sections so that those that are not only deficient but are also hazardous (on the basis of their crash histories), can be singled out for safety improvement. The next section discusses how this was done for Indiana's rural two-lane state highway sections.

\subsubsection{Needs Assessment on the Basis of Both Deficiencies and Hazardousness, with Unlimited Budget (Scenario 2)}

From the list of deficient sections obtained in the previous analysis, the hazardous sections were identified using the Rate-frequency Quality Control method described in the NCHRP 440 report. This method defined a "hazardous" location as a section whose crash frequency and crash rate exceed their respective critical values (see Chapter 3 for Study Methodology of this report). Using this definition, the hazardous locations on the state rural two-lane system were identified. These locations were then ranked in order of their hazardousness. Knowing the specific geometric deficiency and 
predominant crash pattern at each hazardous section, a set of alternative safety improvement projects was identified. Then from this set, the most cost-effective alternative was selected for that section (Table 8-5).

Table E-1, in Appendix E, presents a priority listing (ranking) of selected safety improvement project for Scenario 2 (no budgetary limit, and section that are both deficient and hazardous). As seen from the table, a capital of $\$ 55$ million is required to address safety problems at sections that are both deficient and hazardous. This value represents a tenth of the monetary needs on the basis of deficiencies only. Table 8-5 summarizes the results in Table E-1, showing a summary of the capital required by project type, and the estimated numbers of crashes to be saved (as well their monetary equivalents) if these projects were to be implemented.

Table 8-5 Summary of Capital Requirement by Project Type, Scenario 2

Budgetary Limit: No Limit (Unconstrained)

Basis of Selection: Sections that are both Deficient and Hazardous ${ }^{3}$

\begin{tabular}{|c|c|c|c|c|c|c|}
\hline \multirow[b]{2}{*}{ Improvement Project } & \multirow[b]{2}{*}{ Mileage } & \multirow{2}{*}{$\begin{array}{c}\text { Capital } \\
\text { Required }^{2}\end{array}$} & \multirow{2}{*}{$\begin{array}{l}\text { Estimated } \\
\text { Benefits }^{2}\end{array}$} & \multicolumn{3}{|c|}{ Crash Reduction $^{1}$} \\
\hline & & & & $\begin{array}{l}\text { Fatal/ } \\
\text { Injury }\end{array}$ & PDO & Total \\
\hline $\begin{array}{l}\text { Horizontal Curve Re-alignment } \\
\text { Projects }\end{array}$ & 0.10 & $\$ 77,250$ & $\$ 122,504$ & 0 & 0 & 0 \\
\hline Guardrail Installation Projects & 136.88 & $\$ 25,240,809$ & $\$ 148,843,879$ & 58 & 0 & 58 \\
\hline Shoulder Paving Projects & 4.18 & $\$ 1,416,920$ & $\$ 11,936,245$ & 5 & 12 & 17 \\
\hline $\begin{array}{l}\text { Shoulder Paving and Guardrail } \\
\text { Installation Projects }\end{array}$ & 5.76 & $\$ 3,014,652$ & $\$ 10,792,477$ & 4 & 9 & 13 \\
\hline $\begin{array}{l}\text { Projects to Widen Roadway lane by } 1 \\
\text { ft each direction \& Install guardrail }\end{array}$ & 20.64 & $\$ 4,895,829$ & $\$ 17,342,598$ & 7 & 9 & 16 \\
\hline $\begin{array}{l}\text { Projects to Widen Roadway lane by } 1 \\
\mathrm{ft} \& \text { Widen Shoulder by } 2 \mathrm{ft} \text { in } \\
\text { direction }\end{array}$ & 1.76 & $\$ 308,148$ & $\$ 2,262,099$ & 0 & 3 & 3 \\
\hline $\begin{array}{l}\text { Projects to Widen Roadway lane by } 1 \\
\mathrm{ft} \text { in each directions }\end{array}$ & 14.32 & $\$ 756,096$ & $\$ 5,654,504$ & 2 & 8 & 10 \\
\hline $\begin{array}{l}\text { Projects to Widen Shoulder by } 2 \mathrm{ft} \\
\text { (each direction) \& Install guardrail }\end{array}$ & 28.47 & $\$ 8,731,322$ & $\$ 51,446,985$ & 19 & 25 & 44 \\
\hline \multirow[t]{2}{*}{$\begin{array}{l}\text { Projects to Widen Shoulder by } 4 \mathrm{ft} \\
\text { each direction }\end{array}$} & 42.33 & $\$ 10,352,648$ & $\$ 44,146,487$ & 14 & 71 & 85 \\
\hline & 254.44 & $\$ 54,793,673$ & $\$ 292,547,778$ & 109 & 137 & 246 \\
\hline
\end{tabular}

Not all deficient sections are hazardous, but a hazardous section is necessarily deficient. As such, the rest of the text will use the term "hazardous" section or location to represent a section that is both deficient and hazardous. It must be noted that the amounts indicated in Table $8-5$ is the 
capital required to implement the safety improvements identified as the most cost-effective solution at hazardous location.

The analysis in this section presents the funds needed to address safety problems at all hazardous locations at Indiana's rural two-lane highways.

\subsubsection{Needs Assessment on the Basis of Both Deficiencies and Hazardousness, with Budgetary} Limit: \$1,000,000 per year for 5 years, using Economic Crash Costing Method, Scenario 3

Table 8-6 presents a summary of the physical and monetary needs associated with safety improvements at Indiana's rural two-lane state highways under the conditions that the agency seeks to address safety problems at sections that are not only deficient but are also hazardous as well. Also, it is assumed here that the agency has a budget of 1 million to fix such problems at such highways. Furthermore, this scenario involves the use of the economic method of crash costing, which assigns a relatively lower cost to fatal, injury, and PDO crashes compared to the comprehensive crash costing method.

The results suggest that under this scenario, INDOT can save approximately 166 total crashes (74 fatal/injury and 92 PDO crashes). It is also seen that on the basis of the method of crash costing used, the benefits to be accrued under this scenario, in monetary terms, exceeds the agency costs of safety improvements by a factor of 3 .

Table 8-6 Selected Safety Improvement Projects for Deserving Locations (Scenario 3)

Budgetary Limit: $\$ 1,000,000$ per year for 5 years

Basis of Selection: Sections that are both Deficient and Hazardous ${ }^{3}$

Method of Crash Costing: Economic Method

\begin{tabular}{|c|c|c|c|c|c|c|}
\hline \multirow[b]{2}{*}{ Improvement Project } & \multirow[b]{2}{*}{ Mileage } & \multirow{2}{*}{$\begin{array}{l}\text { Capital } \\
\text { Required }^{2}\end{array}$} & \multirow{2}{*}{$\begin{array}{l}\text { Estimated } \\
\text { Benefits }^{2}\end{array}$} & \multicolumn{3}{|c|}{ Crash Reduction $^{1}$} \\
\hline & & & & $\begin{array}{l}\text { Fatal/ } \\
\text { Injury }\end{array}$ & PDO & Total \\
\hline Install guardrail & 5.22 & $\$ 962,573$ & $\$ 4,885,793$ & 33 & 0 & 33 \\
\hline Install paved shoulder & 0.90 & $\$ 305,078$ & $\$ 929,544$ & 4 & 12 & 17 \\
\hline $\begin{array}{l}\text { Widen Roadway lane by } 1 \mathrm{ft} \& \text { Widen } \\
\text { Shoulder by } 2 \mathrm{ft}\end{array}$ & 0.33 & $\$ 57,777$ & $\$ 229,956$ & 1 & 4 & 5 \\
\hline Widen Roadway lane by $1 \mathrm{ft}$ & 56.28 & $\$ 2,971,584$ & $\$ 8,071,815$ & 31 & 62 & 92 \\
\hline \multirow[t]{2}{*}{ Widen Shoulder by $4 \mathrm{ft}$} & 2.83 & $\$ 692,133$ & $\$ 1,626,289$ & 5 & 14 & 19 \\
\hline & 65.56 & $\$ 4,989,146$ & $\$ 15,743,400$ & 74 & 92 & 166 \\
\hline
\end{tabular}

1. Rounded off to nearest whole number

2. Costs and Benefits are in Year 2000 constant dollar

3. Method used for Identifying both Deficient and Hazardous Sections - Integrated Quality Control Method 


\subsubsection{Needs Assessment on the Basis of Both Deficiencies and Hazardousness, with Budgetary Limit: \$1,000,000 per year for 5 years, using Comprehensive Crash Costing Method, Scenario 4}

Table 8-7 summarizes the types of physical safety improvements needed and their associated monetary costs for sections at the state's rural two-lane state highways that are deemed both deficient and hazardous. An annual budgetary constraint of 1 million is assumed, and comprehensive crash costing is used in this scenario. It is seen that over 180 crashes will be saved if this scenario is used ( 74 fatal/injury and 104 PDO crashes). For a million dollar budget and on the basis of sections prioritized by their hazardousness as well as their geometric deficiencies, it is seen that the benefits of safety improvements in terms of crash reduction seem to be higher for all crashes (but same for fatal crashes) when INDOT uses the comprehensive crash costing method compared to the economic crash costing method. On the other hand, in terms of crash reduction per dollar, use of the comprehensive method yields approximately $50 \%$ lower cost-effectiveness compared to the economic method (approximately 1.5 vs. $3)$.

Table 8-7 Summary of Capital Requirement by Project Type (Scenario 4)

Budgetary Limit: $\$ 1,000,000$ per year for 5 years Basis of Selection: Sections that are both Deficient and Hazardous ${ }^{3}$

Method of Crash Costing: Comprehensive Method

\begin{tabular}{|l|c|c|c|c|c|c|}
\hline & \multirow{2}{*}{ Improvement Project } & Mileage & $\begin{array}{l}\text { Capital } \\
\text { Required }\end{array}$ & \multirow{2}{*}{$\begin{array}{l}\text { Estimated } \\
\text { Benefits }^{2}\end{array}$} & \multicolumn{3}{|l|}{$\begin{array}{l}\text { Frash Reduction } \\
\text { Injury }\end{array}$} & PDO & Total \\
\hline Install guardrail & 5.22 & $\$ 962,573$ & $\$ 2,165,619$ & 29 & 0 & 29 \\
\hline Install paved shoulder & 0.71 & $\$ 240,672$ & $\$ 386,560$ & 4 & 12 & 17 \\
\hline $\begin{array}{l}\text { Widen Roadway lane by 1 ft \& Widen } \\
\text { Shoulder by 2 ft }\end{array}$ & 0.33 & $\$ 57,777$ & $\$ 114,488$ & 2 & 4 & 6 \\
\hline Widen Roadway lane by 1 ft & 56.16 & $\$ 2,965,248$ & $\$ 3,868,762$ & 33 & 67 & 101 \\
\hline Widen Shoulder by 2 ft & 0.59 & $\$ 72,147$ & $\$ 71,143$ & 1 & 5 & 6 \\
\hline Widen Shoulder by 4 ft & 2.83 & $\$ 692,133$ & $\$ 818,842$ & 5 & 16 & 21 \\
\hline
\end{tabular}

1. Rounded off to nearest whole number

2. Costs and Benefits are in Year 2000 constant dollar

3. Method used for Identifying both Deficient and Hazardous Sections - Integrated Quality Control Method 


\subsubsection{Needs Assessment on the Basis of Both Deficiencies and Hazardousness, with Budgetary Limit: \$2,000,000 per year for 5 years, using Economic Crash Costing Method, Scenario 5}

A summary of the physical and monetary needs associated with safety improvements at Indiana's rural two-lane state highways is presented as Table 8-8. The assumptions here are that INDOT seeks to address safety problems at sections that are not only deficient but are also hazardous as well; that INDOT has a budget of 2 million to fix such problems at such highways; and that economic method is used for crash costing.

The results suggest that under this scenario, INDOT can save approximately 244 total crashes (126 fatal/injury and 120 PDO crashes). It is also seen that on the basis of the method of crash costing used, the benefits to be accrued under this scenario, in monetary terms, exceeds the agency costs of safety improvements by a factor of 2.7. It is seen that all other conditions remaining the same, doubling the budget significantly increases the benefits in terms of crash reduction and crashes saved, but leads to less-than-double the cost-effectiveness or crashes saved. This suggests that infinitely increasing the budget for physical road improvements is not likely to completely eradicate the crash problem at rural two-lane highways. In other words, there seems to be a ceiling to the effectiveness of engineering safety countermeasures. As such, non-engineering countermeasures such as safety education and enforcement are also vital to reduce the crashes that are associated with problems from drivers, vehicles, and the environment.

Table 8-8 Summary of Capital Requirement by Project Type (Scenario 5)

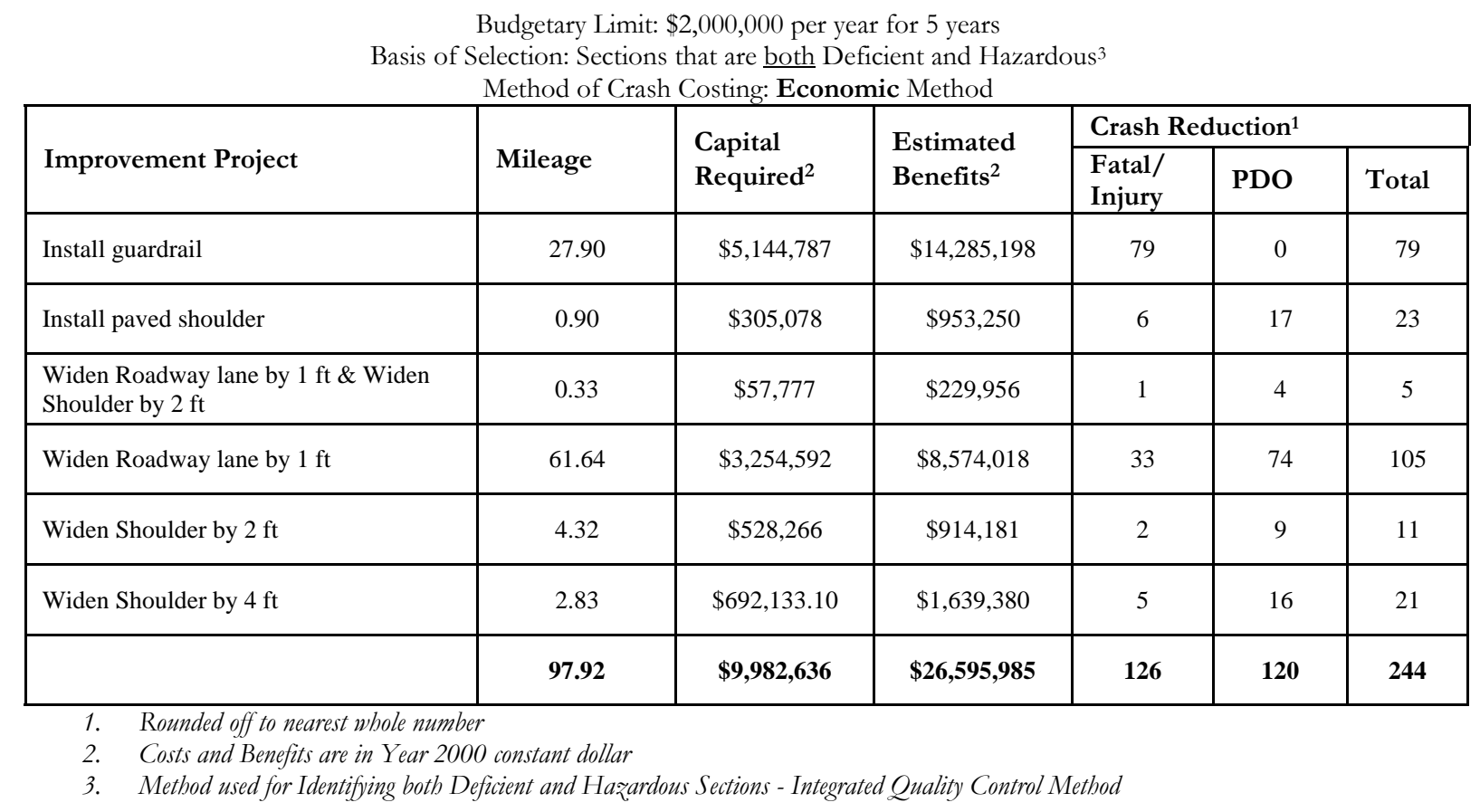




\subsubsection{Needs Assessment on the Basis of Both Deficiencies and Hazardousness, with Budgetary}

\section{Limit: \$2,000,000 per year for 5 years, using Comprehensive Crash Costing Method, Scenario 6}

Table 8-9 presents a summary of the physical and monetary needs associated with safety improvements under the assumptions similar to those of Scenario 6 with the exception that the comprehensive method is used for crash costing. The results suggest that under this scenario, INDOT can save approximately 243 total crashes (127 fatal/injury and 120 PDO crashes). It is also seen that on the basis of the method of crash costing used, the benefits to be accrued under this scenario, in monetary terms, exceeds the agency costs of safety improvements by a factor of 1.2 (less than the 1.5 cost-effectiveness value for the $1 \mathrm{~m}$ budget). Again it is seen that all other conditions remaining the same, doubling the budget significantly increases the benefits in terms of crash reduction and crashes saved, but leads to less-than-double the cost-effectiveness or crashes saved. Again, this suggests that infinitely increasing the budget for physical road improvements is not likely to completely eradicate the crash problem at rural two-lane highways. As such, regardless of crash costing method used, there seems to be a ceiling to the effectiveness of engineering safety countermeasures, and further corroboration is lent to the importance of non-engineering countermeasures if the safety problem at Indiana's rural two-lane highways is to be addressed fully. Another interesting finding is that as the budget increases, the needs assessment results using the two alternative crash costing methods differ in terms of the estimated total monetary benefits but seem to converge in terms of cost-effectiveness and crashes saved.

Table 8-9 Summary of Capital Requirement by Project Type (Scenario 6)

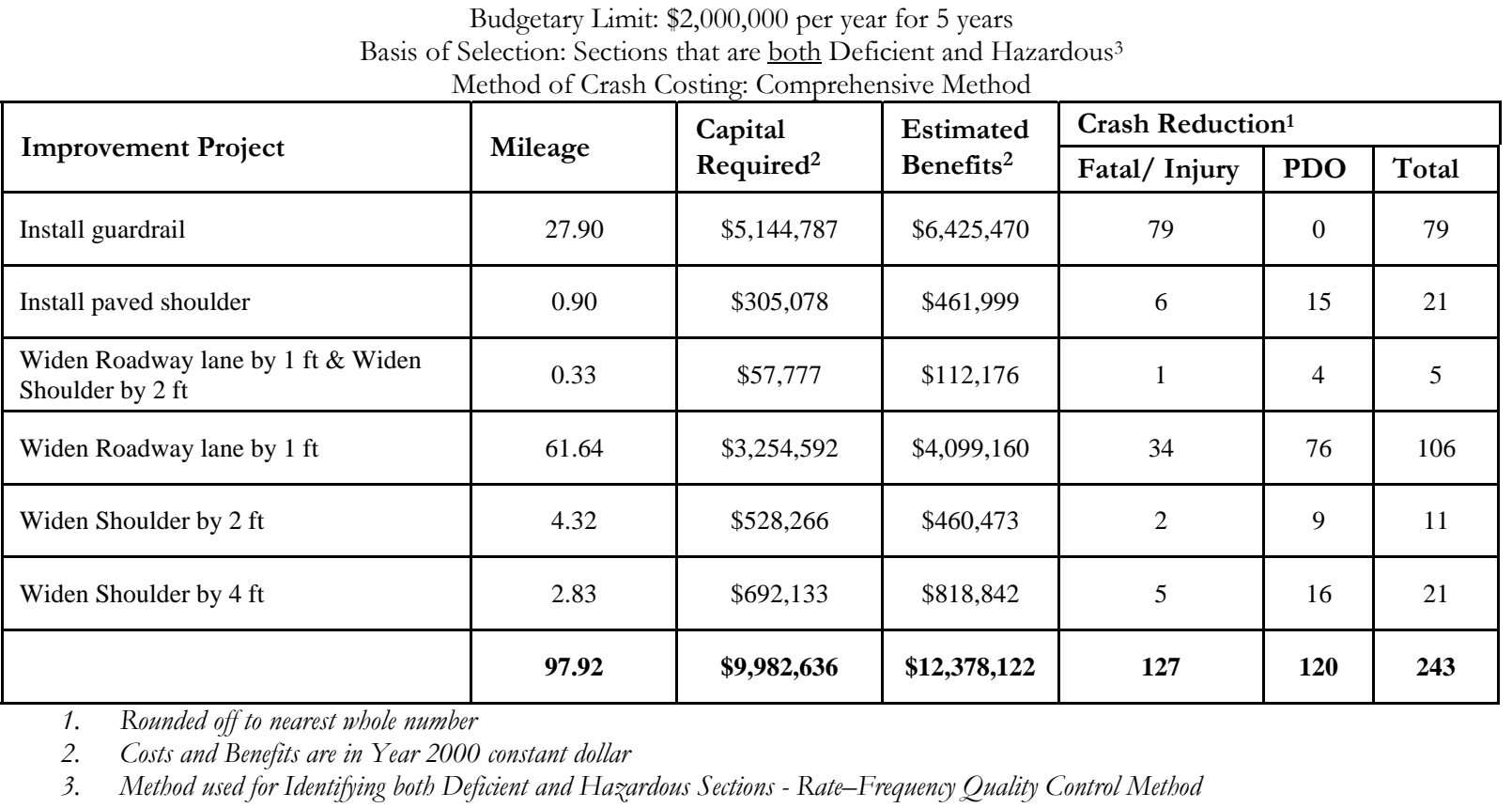




\subsection{CHAPTER SUMMARY}

Unlike previous chapters that focused on the effectiveness and cost-effectiveness of individual treatments, the present chapter addresses a system-wide safety management issue by considering the entire network of Indiana's two-lane rural state highway network, identifying deficient and hazardous sections on the network, determining the most cost-effective countermeasure at each section, and prioritizing all such needed safety improvements for the entire network over an analysis period and within a given budgetary constraint. For each year within the analysis period, the chapter assessed the physical needs (where are improvements needed and what kind?) and monetary needs (how much is needed to address identified physical needs?).

Under several scenarios that differ by alternative budgetary ceilings for rural two-lane state highways and crash costing method, the chapter utilized optimization procedures to identify where budgeted funds could be best used so that overall cost-effectiveness is maximized. For rural two-lane state highway sections that are deficient but not necessarily hazardous, it was found that $\$ 530$ million would be needed to address all engineering safety needs. On the basis of sections that are not only deficient but are also hazardous, it was determined that $\$ 55$ million is required. For each scenario, the chapter provided summaries of capital requirement for addressing safety at the state's rural two-lane state highways and the benefits to be accrued in terms of the number of crashes saved, the total value of crashes saved, and the cost-effectiveness of the investment (ratio of the total value of crashes saved to the total agency cost of the safety improvements).

The results in this chapter also showed that as the budget increases, the needs assessment results using the two alternative crash costing methods differ in terms of the estimated total monetary benefits but seem to converge in terms of cost-effectiveness and crashes saved.

Finally, findings in this chapter seem to suggest that infinitely increasing the budget for physical road improvements is not likely to completely eradicate the crash problem at rural two-lane highways. In other words, there is a ceiling to the effectiveness of engineering safety countermeasures, and therefore non-engineering countermeasures such as safety education and enforcement are also vital to reduce the crashes that are associated with problems from drivers, vehicles, and the environment. 


\section{CHAPTER 9 SUMMARY AND CONCLUSIONS}

This study was carried out in response to a need stated by INDOT and LTAP to update/investigate the relationships between crash rates and various geometric conditions at two-lane rural highways and county roads in the state of Indiana. With such relationships, these agencies intend to carry out more reliable assessments of physical improvements on rural two-lane road safety in terms of crash reduction and cost-effectiveness, and also to prioritize safety investments for various

links on the rural two-lane road networks. This way, the agencies can determine the impacts of various funding levels on changes in fatal, injury and PDO crashes. Such information would assist state and local decision-makers in safety resource allocation, and also to evaluate the safety implications of alternative levels of specific safety investments on such highway segments. The application of the results to practice is expected to lead to more productive use of such highways, better allocation of resources of engineering-related safety investments, and more effective management of crash risks.

The study evaluated the safety effects of geometric and other characteristics at rural two-lane highways and county roads in the state of Indiana. For rural two-lane highways, three classes were considered: rural major collectors, rural minor arterials, and rural principal arterials. This classification reflects the significant differences in the interactive effects of mobility and accessibility that are not explicitly addressed by any available quantitative variables. Also, the classification levels serve as qualitative variables (truck percentage, speed limits, speed variance, access control, etc.) that surrogate for certain traffic characteristics and geometric design standards which were not included in the modeling process due to lack of data or other reasons.

The study begun with a detailed literature survey regarding the crash trends and factors from published information. The next step was to carry out a survey of law enforcement officials to identify any road locations that are prone to crashes. Then data were collected for the sate and local rural two-lane systems. For county roads, crash and volume data were obtained from Tippecanoe County LTAP office, and data on road and shoulder width and surface types, number of curves and driveways, etc. were collected on-site by the Research Team. For state highways, crash, traffic and inventory data were obtained from INDOT and CATS. 
Preliminary descriptive analysis of the data was carried out to identify general trends in the selected variables. Detailed statistical analyses were conducted to develop crash prediction models from which crash reduction factors were developed. Then for the state highway rural two-lane highways, the cost-effectiveness values of various countermeasures were determined as a function of the countermeasure cost and crash reduction. Also, the crash reduction factors and costs of the countermeasures were used, together with the existing inventory of roads and their geometric deficiencies, to carry out financial needs assessments for various budgeting and crash costing scenarios for the rural two-lane state highway system. The study results are summarized in the following sections, and an overall concluding discussion and implementation issues are provided thereafter.

\subsection{FACTORS AFFECTING CRASH OCCURRENCE}

For each state highway functional class, crash prediction models were developed for four crash severity categories-- Injury crashes, PDO crashes, Fatal+Injury crashes, and Total crashes.

The Section Length coefficient showed that longer road sections are expected to have more crashes, but fewer crashes per mile compared to shorter ones, all other factors being the same. However, increasing the length of a section with the intention of lowering crash rates is not a feasible option and was therefore not considered as a countermeasure and thus no crash reduction factor was established for the section length variable.

Similarly, Traffic Volume (AADT) turned out to be a significant variable in all the crash models for all road classes: higher traffic volume generally translates to higher number of crashes. However, higher traffic volume generally leads to lower crashes per AADT. It is interesting to note that beyond a certain point, increasing traffic leads to decreasing crashes and very sharp decreases in crashes per AADT, which is suggestive of congestion effects. Again, raising traffic levels for the purpose of reducing crash rates is not a viable engineering option and therefore crash reduction factors were not developed for this crash factor.

The Lane Width variable was found to be a significant factor in all the crash models, and increasing lane width was generally found to lead to decreased crashes, largely because wider lanes serve as buffer zones that offer greater opportunity for recovery of errant vehicles or for avoiding errant oncoming vehicles.

Increasing Shoulder Width is generally associated with decreasing in number of crashes, all other factors remaining the same, obviously because for rural two-lane roads, wider shoulders enhance safety by providing additional buffer zone where operators of stray vehicles can regain 
control, recover from error and subsequently resume normal travel. The study found that increasing the shoulder width generally leads to greater reduction in fatal and injury crashes compared to noninjury crashes.

Pavement Friction was found to be a significant variable for the relatively lower classes of roads -- rural major collectors and minor arterials. Sections with higher pavement friction were found to experience lower crash rates, obviously because pavement friction helps to reduce the stopping distance required by vehicles and lower the chances for crashes particularly of the rear-end types. The effect of pavement friction was however found to be insignificant in the crash models for the highest class of rural two-lane roads (principal arterials) possibly due to the relatively little variability in friction numbers for that road class.

Pavement Condition is significant for rural principal arterials, but not for the two lower road classes. It was seen that principal arterials with poor pavement condition seem to experience more frequent crashes. Poor surface condition impedes the ability to control vehicles in the safest way possible. Also, in severe cases of surface distress, drivers seeking to avoid a pavement defect may stray onto the adjacent lane (into the path of oncoming vehicles) or may stray onto the roadside where they may encounter a crash situation

The quality of horizontal alignment, represented by the Average Horizontal Curve Radius, was found to be indirectly related to crashes. A higher average curve radius was found to be associated with fewer crashes. An adequate radius provides smoother transition between tangent section and leads to reduction of centrifugal forces on vehicles negotiating the curve, thereby reducing the risk of overturning. Also, higher quality of vertical alignment, represented by a lower Average Vertical Grade, was found to be associated with reduced crashes: lower vertical grades improves sight distance and enhances safety.

A number of factors investigated for rural two-lane county roads were not considered in the analysis for rural two-lane state highways due to lack of such data or lack of variability in the factors. For example, Shoulder Type variable was investigated for county roads, and it was seen that increasing quality of shoulder (from no shoulder to gravel/grass to asphalt) is associated with decreasing crash frequency. This is expected, because improved shoulder surfaces enhance the grip of vehicle tires. Also for county roads, Driveway Density was found to be an influential factor: a greater number of driveways per mile is associated with higher crash frequency. Also, county roads that had any speed limit posting, regardless of level of speed posted, were associated with higher crashes. While this result seems to be contrary to expectation, it may be explained that such postings at county roads 
indicate driving speeds that are generally higher than safe speeds implicitly permitted by existing geometrics at those sections. The value of the posted speed was not considered.

\subsection{TRENDS IN CRASH REDUCTION FACTORS}

Lane width: With respect to PDO crashes, lane width increases was found to be most effective at rural principal arterials and least effective in at rural major collectors. On the other hand, for fatal and injury crashes, increase in lane width is most effective at rural major collectors and least effective for rural principal arterials. It can therefore be seen that as the class of road decreases, relatively higher percentages of severe crashes and relatively lower percentage of non-severe crashes, are saved by increases in lane width.

Shoulder Width: For PDO crashes, increase in shoulder width up to $5 \mathrm{ft}$ is most effective at rural principal arterials but least effective in at rural major collectors. Also, for fatal and injury crashes, increase in shoulder width is most effective at rural principal arterials but least effective for rural principal arterials. The result suggests that as the class of road increases, relatively higher percentages of crashes of all severities are saved by increases in shoulder width. This finding applies for roads on the state highway system. For county roads, increase in shoulder width seems to have the same margin of effect as it does for rural principal arterials.

Skid Resistance: For PDO crashes, increase in skid resistance width is more effective at rural major collectors but less effective at rural minor arterials. On the other hand, for fatal and injury crashes, increase in skid resistance is more effective at rural minor arterials but least effective for rural major collectors. It can therefore be seen that as the class of road increases, relatively higher percentages of severe crashes and relatively lower percentage of non-severe crashes, are saved by increases in skid resistance.

Horizontal Alignment Improvement. For PDO crashes, increase in horizontal curve radius is most effective at rural minor arterials but least effective in at rural major collectors. Also, for fatal and injury crashes, increase in lane width is most effective at rural minor arterials but least effective for rural major collectors. Obviously, for the two extreme road classes (major collectors and principal arterials), relatively smaller percentages of all crash severity types are saved by improvements in horizontal alignment, compared to rural minor arterials. County roads seem to stand to benefit most from horizontal alignment improvements.

Vertical Alignment: For PDO crashes, increase in the quality of vertical alignment (decrease in grade) is most effective at rural major collectors but least effective in at rural principal arterials. Also, fatal and injury crashes, increase in quality of vertical alignment is most effective at rural major collectors but 
least effective for rural principal arterials. This finding suggests that as the class of road decreases, relatively higher percentages of all crash severity types are saved by improvements in vertical alignment.

Other factors: Due to lack of data, there were a number of countermeasures whose crash reduction factors could not be developed in the present study. As shown in the literature review of this report (Chapter 3), nationwide studies have investigated these countermeasures and have developed crash reduction factors which can, in the interim, be used for evaluating safety improvements at INDOT or LTAP. These include providing increased opportunity for passing, increased bridge width, provision of superleveation, provision of shoulder or centerline rumble strips, removal of roadside obstacles, provision of animal crossing facilities, etc.

\subsection{COST-EFFECTIVENESS OF SAFETY ENHANCEMENTS}

\section{Cost-effectiveness by Extent of Countermeasure}

Lane Width: For injury and PDO crashes rural major collectors, increasing the lane width of Indiana's rural major collectors is associated with increasing cost-effectiveness up to a $2 \mathrm{ft}$ increase in lanewidth, and a decreasing cost-effectiveness thereafter. For fatal crashes, increases in lane-width are consistently associated with lowered cost-effectiveness. Beyond $3 \mathrm{ft}$ of additional lane-width, the decrease in cost-effectiveness for all crash severity types seems to flatten out. For rural minor arterials, the general cost-effectiveness trends were found to be similar to those of rural major collectors. Increasing the lane width of Indiana's rural minor arterials is associated with generally decreasing cost effectiveness up to a $2 \mathrm{ft}$ increase in lane-width, and a rather sharply decreasing costeffectiveness thereafter, but does not flatten out as is the case for rural major collectors. The patterns of cost-effectiveness were generally similar to those of rural major collectors and minor arterials. Increasing the lane width of Indiana's rural minor arterials is associated with gradual decreases in cost-effectiveness.

Shoulder Width: For shoulder width increases at rural major collectors, the results seemed to be equivocal: for PDO crashes, shoulder widening cost-effectiveness seems to increase up to $2 \mathrm{ft}$ of widening, and drops gently thereafter. For the other crash severity types, very gentle or no drops in cost-effectiveness is observed for increasing shoulder width. In the case of rural minor arterials, it is also seen that cost-effectiveness gently decreases with increasing extension in shoulder width. For rural principal arterials, it is seen that cost-effectiveness gently decreases with increasing shoulder width up to $5 \mathrm{ft}$ extension, and decreases sharply thereafter. 
Pavement Surface Friction: With regard to pavement surface friction at rural major collectors and minor arterials, surface treatments are known to offer significant benefits directly in terms of skid resistance. The study showed that for all three state highway classes, chip sealing is generally twice as costeffective (but less effective) compared to micro-surfacing in addressing friction problems at rural major collectors.

Pavement Surface Condition: For pavement surface friction improvements at rural principal arterials, cost-effectiveness decreases with increasing effort, varying significantly by crash severity type but converges with increasing effort.

Horizontal Curve Radius: For rural major collectors, the effectiveness trends for increasing horizontal curve radius were mixed, but generally followed a trend of decreasing cost-effectiveness with increasing enhancements to horizontal curve radius. In the case of rural minor arterials, for all crash severity types, increasing horizontal radius is associated with gently decreasing cost effectiveness. Increasing efforts to improve horizontal alignment at rural principal arterials (represented by increasing its radius) is associated with gently decreasing cost effectiveness, for all crash severity types.

Vertical Alignment Quality: Increases in vertical alignment quality were found to be generally associated with increased cost-effectiveness.

\section{Discussion}

The generally decreasing cost-effectiveness of the above treatments with increasing effort seems to reflect the fact the increasing efforts leads to disproportionate increases in costs. For example, in order to widen a lane or shoulder, or to reduce horizontal curve radius, a significant amount of money is needed to remove topsoil, fill the existing drain with approved material, fill the relevant portion of low-lying surrounding terrain to finished road level, and laying/compacting of pavement material. If more width is needed, the agency moves from an already acquired right-of-way into abutting land and thus additional cost is incurred in acquiring additional right-of-way and to relocate or demolish existing fence wall and other structures within the right-of-way. Such increases in costs as the extent of road or shoulder widening increases, is obviously enough to offset any economies of scale arising from increased work output, and leads to decreased cost-effectiveness of such increasing efforts. In the case of friction number enhancement, large increases in friction number does not involve additional purchases of ROW, but involves a surface treatment (micro-surfacing) that is over 
4 times as costly as the less expensive option (chip sealing) but is only 2-3 times more effective in improving pavement condition. For increasing quality of vertical alignment (decreasing vertical grade) an increase in improvement effort (through further reductions in vertical grade) is associated with increasing cost-effectiveness. Similar to the case for horizontal curve improvements, vertical alignment improvements are achieved through capital intensive work such as excavation and filling. However, unlike the case for horizontal curves, additional right-of-way is typically not required for vertical alignment enhancements, as such increasing enhancement efforts do not translate to significant increases in costs. It is therefore not surprising to see that increasing enhancements in vertical alignment is associated with increasing cost-effectiveness. This relationship is more pronounced for PDO crashes and less pronounced for fatal and injury crashes.

\section{Cost-effectiveness by Type of Countermeasure}

As stated elsewhere in this report, it may not be very meaningful to compare the cost-effectiveness across different countermeasures except in the following situations: (i) the countermeasures are viable mutually exclusive alternative actions that remedy a given safety problem at an existing road, or (ii) an existing road suffers from multiple types of safety problems so many different countermeasures may be carried out and funds are available to carry out only few of such countermeasures. Skid resistance (friction) improvement appears to be most cost-effective in terms of the percentage of crashes reduced $(2-5 \%$ of existing crashes saved per $\$ 10,000$ expended in that effort). It was also seen that vertical grade enhancement has higher cost-effectiveness than horizontal alignment improvements. Another interesting case is that of lane widening and shoulder widening. Faced with a narrow road, agencies are typically faced with a dilemma of whether to increase the lane-width or to increase the shoulder width. High costs are encountered in lane extensions (due to high cost of pavement surfacing material), but the cost data suggests that such costs are outweighed by the extra right-of-way acquisition costs that are often associated with shoulder extensions. Besides, a unit increase in shoulder width, as seen from the previous section of this chapter, has a lower effectiveness (lower crash reduction) than a unit increase in lane width. As such, shoulder extensions are less cost-effective compared to lane additions. The analysis suggests that agencies generally stand to reap, on the average, three times as many returns per dollar if they choose to widen an existing lane rather than adding a shoulder. It must be noted that these are average conditions only. Thus for a road with lanes that are already very wide and no shoulder, it is generally more worthwhile to provide a shoulder rather than extend the existing lane. 


\section{Cost-effectiveness by Crash Severity Type}

Lane width extensions at rural major collectors seem to be associated with higher cost-effectiveness for fatal and injury crashes compared to PDO crashes. At rural minor arterials however, costeffectiveness of lane widening had relatively little differences across crash severity types. For rural principal arterials, lane widening is associated with significant differences in cost-effectiveness across crash severity types. For all highway classes, the patterns of changing cost-effectiveness as lane width increases, seems to fairly similar across all crash types. For shoulder-widening treatments and horizontal curve enhancements, change in cost-effectiveness per increasing lane width seems to fairly similar across all crash types at rural major collectors but are markedly different at rural minor arterials. For shoulder widening at rural principal arterials, cost-effectiveness levels are relatively higher for fatal and injury crashes compared to PDO crashes. For surface friction improvement at rural major collectors, the level and sensitivity of the treatment seemed to be uniform across all crash severity types. For surface friction improvements at rural minor arterials, cost-effectiveness levels are relatively higher for fatal and injury crashes compared to PDO crashes. For pavement condition improvements at rural principal arterials, cost-effectiveness levels are relatively higher for fatal and injury crashes compared to PDO crashes. Vertical grade improvements at rural major collector and minor arterials appear to be more cost-effective for PDO crashes and less cost-effective for fatal and injury crashes. For horizontal and vertical alignment improvements at rural principal arterials, costeffectiveness levels are higher for PDO crashes compared to fatal and injury crashes.

\section{Cost-effectiveness Findings across Road Classes}

While the trends in cost-effectiveness relative to treatment effort were generally similar across the three classes of state highways, there were a few differences in cost-effectiveness levels across them. On the basis of PDO crashes, increases in lane width seem to be more cost-effective at rural principal arterials (approximately 1.9\% crash reduction per \$10,000) than the other road classes. On the basis of fatal and injury crashes, there seemed to be generally similar average cost-effectiveness levels across the road classes. Also, increases in shoulder width up to $5 \mathrm{ft}$ are most cost-effective at rural

principal arterials and least cost-effective at rural major collectors. It is also seen that on the basis of fatal and injury crash reduction per dollar, friction improvement has a greater cost-effectiveness at rural minor arterials compared to rural major collectors. However, on the basis of PDO crash reduction per dollar, friction improvement has a lower cost-effectiveness at rural minor arterials compared to rural major collectors. Horizontal curve improvements seem to have the highest costeffectiveness at rural minor arterials and the least cost-effectiveness at rural collectors. Also, cost- 
effectiveness of horizontal curve improvements at rural minor arterials appear to be more sensitive to increases in the extent of this treatment compared to rural major collectors or principal arterials. Furthermore, at rural major collectors horizontal curve improvement has a greater cost-effectiveness in terms of fatal and injury crashes compared to PDO crashes, while the reverse is true for rural minor arterials and principal arterials. Vertical alignment improvement cost-effectiveness levels were similar for rural major collectors and minor arterials and were higher than those for rural principal arterials. It is interesting to note that for rural major collectors and principal arterials, fatal and injury crashes have similar levels of vertical alignment improvement cost effectiveness while for rural minor arterials, this treatment is more cost-effective for fatal crashes than it is for injury crashes.

\subsection{SAFETY NEEDS ASSESSMENTS}

An assessment of the monetary needs to address the physical problems on the state's rural two-lane system was carried out on the basis of sections that are both geometrically deficient and hazardous. For cases where budgetary constraints were considered (thereby making it necessary to incorporate crash costs), needs assessments was carried out separately on the basis of economic costs and comprehensive methods of crash costing. Needs assessments were carried out only for the state highway system and not for the county road system due to lack of costs of safety countermeasure sand crashes at county roads. The needs assessment was carried out using the Indiana Safety Management System software package. The countermeasure effectiveness (crash reduction factors) used in the software were updated with values obtained in the present study.

For sections that are deficient but not hazardous, it was found that $\mathbf{\$ 5 3 0}$ million (in Year 2000 constant dollars) would be needed to address deficiencies to their design standards. This is based on the inventory as of July, 2001. At the time of reporting, the financial needs are expected to be slightly less than that shown as a few more sections may have been upgraded to eliminate such deficiencies between July 2001 and the time of reporting. An analysis based only on existing geometric deficiencies, albeit simple, may not be appropriate because (i) a deficient section is not necessarily hazardous, and (ii) the prohibitive cost of addressing all deficient sections on Indiana's rural two-lane sections renders such solution impractical. As such, the study also investigated sections that are not only deficient but are also hazardous (on the basis of their crash histories) and determined that $\$ 55$ million is required to address safety problems at sections that are deemed both deficient and hazardous. Assuming a limited budget of $\$ 1 \mathrm{M}$ per year over a 5-year period, it was found that 170-180 crashes (translating to $\$ 8-15$ million) can be saved (the indicated range is due to varying values yielded by economic and comprehensive methods of crash costing). If the annual budget is increased to $\$ 2 \mathrm{M}$ per year over a 5 -year period, it was found that 244 crashes (translating to $\$ 12-26$ million) can be saved. 
The study results showed that as the budget increases, the needs assessment results using the two alternative crash costing methods differ in terms of the estimated total monetary benefits but seem to converge in terms of cost-effectiveness and crashes saved.

Finally, this study showed that infinitely increasing the budget for physical road improvements is not likely to completely eradicate the crash problem at rural two-lane highways. In other words, there is a ceiling to the effectiveness of engineering safety countermeasures, and therefore non-engineering countermeasures such as safety education and enforcement are also vital to reduce the crashes that are associated with problems from drivers, vehicles, and the environment.

\subsection{CONCLUDING REMARKS}

Most two-lane rural highways and county roads in Indiana have operational and safety deficiencies arising from inadequate road geometry, driver information deficiencies, lack of passing opportunities, and traffic conflicts due to driveways (Plates 9-1 and 9-2). Most of such roads were designed and built many decades ago using standards that have become outdated vis-à-vis current traffic trends and design policy. As the present study has shown, there is need to replace/rehabilitate pavements, widen and/or pave lanes and shoulders, improve horizontal and vertical alignments, among others. It is therefore not surprising that the safety performance of rural two-lane roads (which account for $70 \%$ of the state highway network), is least compared to all other road classes when considered on the basis of crash rates (crashes per vehicle-miles traveled).

Overall, for most road classes, crash rates for all crash severity types generally declined over the 1997-2000 period obviously due to increased traffic volume, and also possibly because of increased enforcement and education, and engineering investments (implementation of physical safety projects). Nevertheless, current numbers are still at levels that may be deemed unsatisfactory and worthy of concern and intervention. Safety countermeasures are categorized into three groups: engineering (that largely addresses highway geometry deficiencies and to some extent, the environment), enforcement (that address driver and vehicle deficiencies) and education (that indirectly addresses all deficiencies). While the present study provides information to INDOT and LTAP for are useful for engineering decisions, these agencies may very well continue to pursue holistic approaches to the safety problem by also supporting regulations for enhanced vehicle worthiness and safety features, sober and responsible driving, and reasonable speed limit policies.

Information made available by the present study would help INDOT and LATP assess the effectiveness and cost-effectiveness of various safety improvements at their rural two-lane roads, and also to carry out a network level needs assessment and priority listing of safety investments at various sections and at various years. 
The safety problems at rural two-lane highways cannot be ignored. As has been stated in previous research, allowing the roads to operate under their current geometric deficiencies would lead to increased fatalities, injuries and property damage crashes, vehicle operating costs, delays and inconveniences which may translate into driver frustration and lost time and these may in turn exacerbate existing safety problems that already exist due to inadequate geometry. There is also the growing problem of tort liability. Even though design and maintenance decisions based on budgetary or other economic constraints are generally seen as discretionary in nature and consequently are generally immune from tort suits, it has been demonstrated in past cases that a transportation agency can be held liable if it fails to present evidence on planning and ordering of priorities. The provision of evidence on planning and programming of investments is an element of pre-emptive risk management that is implicitly associated with any overall safety management program and would place INDOT and LTAP in a better position to defend themselves against tort claims related to highway geometry or other engineering features. Prioritization of safety investments through planning and programming is necessary because the state cannot afford the $\$ 0.5$ billion (as determined from the present study) that is needed to fix all geometric deficiencies at the rural two-lane highways (and probably a similar amount for the county road network).

Information made available by the present study would help INDOT and LTAP assess the effectiveness and cost-effectiveness of various safety improvements at their rural two-lane roads, and also to carry out a network level needs assessment and priority listing of safety investments at various sections and at various years. The present study provides data on safety countermeasure effectiveness and cost-effectiveness. These data are vital inputs in the development of a safety management program that identifies problems on the Indiana's rural two-lane highway system, develops safety needs assessments and priority schedules for safety investment, and assess the impacts of alternative safety action and funding levels.

Another issue of concern is that of geriatric driving. At the present time, the need to address current safety deficiencies at the state's rural two-lane roads takes on an urgent dimension that ever before. As the baby boom generation reaches retirement age, a large wave of geriatric drivers is expected. Past studies have shown that while the overall traffic fatalities have remained relatively constant over the past 10 years, the number of older drivers killed on the nation's highways has been on the rise [Public Roads, 2004]. As such, it is important to identify roadway improvements that would make driving easier and safer for older drivers, particularly at rural two-lane roads. Such improvements include increased lane and shoulder width, better markings, visible signs, etc. The results of the present study make it possible for INDOT to identify critical areas of improvement needed on the state's rural two-lane system, and the costs and benefits associated with such improvements. 


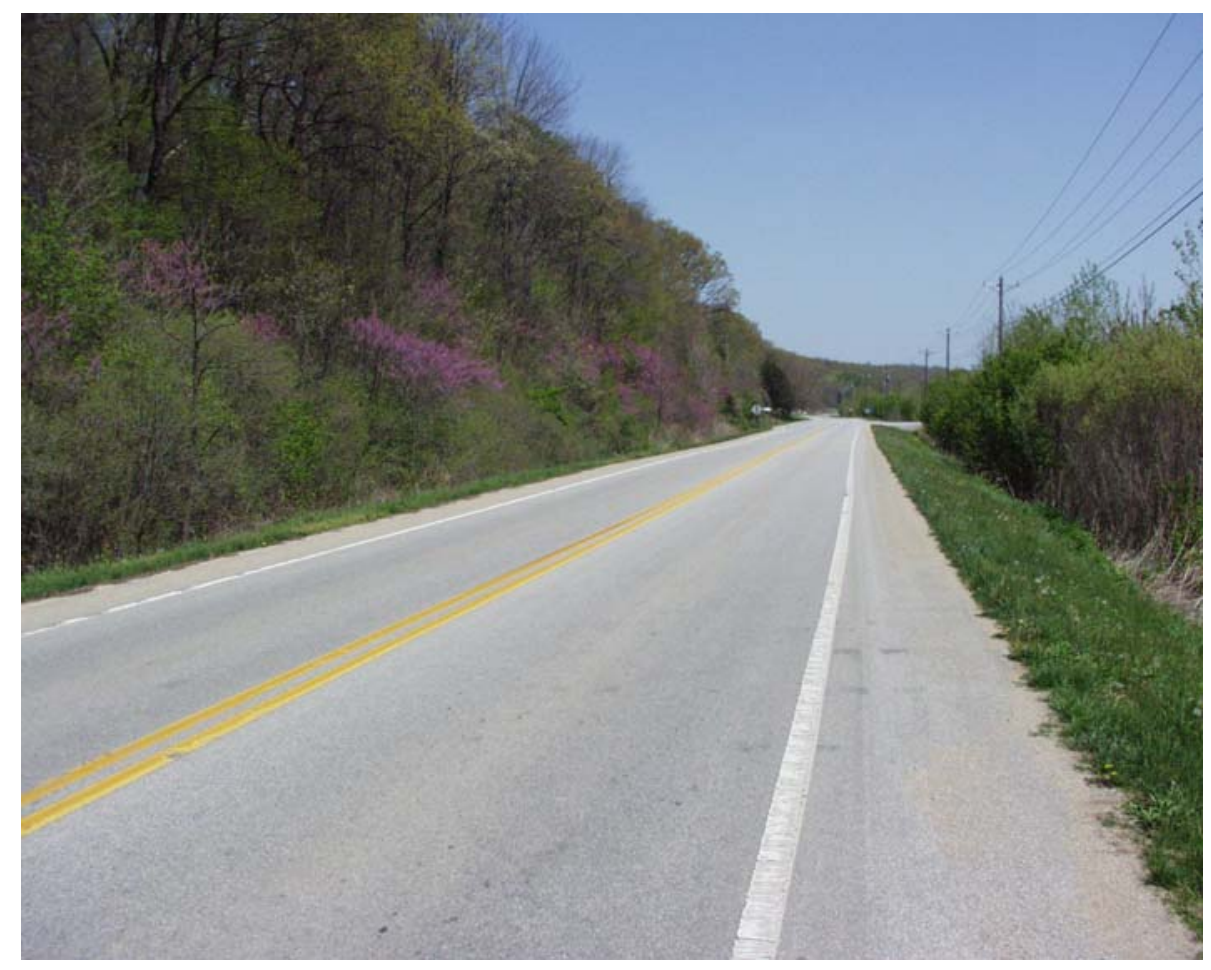

Plate 9-1: Only Few County Roads Have Adequate Geometrics and Pavement Markings

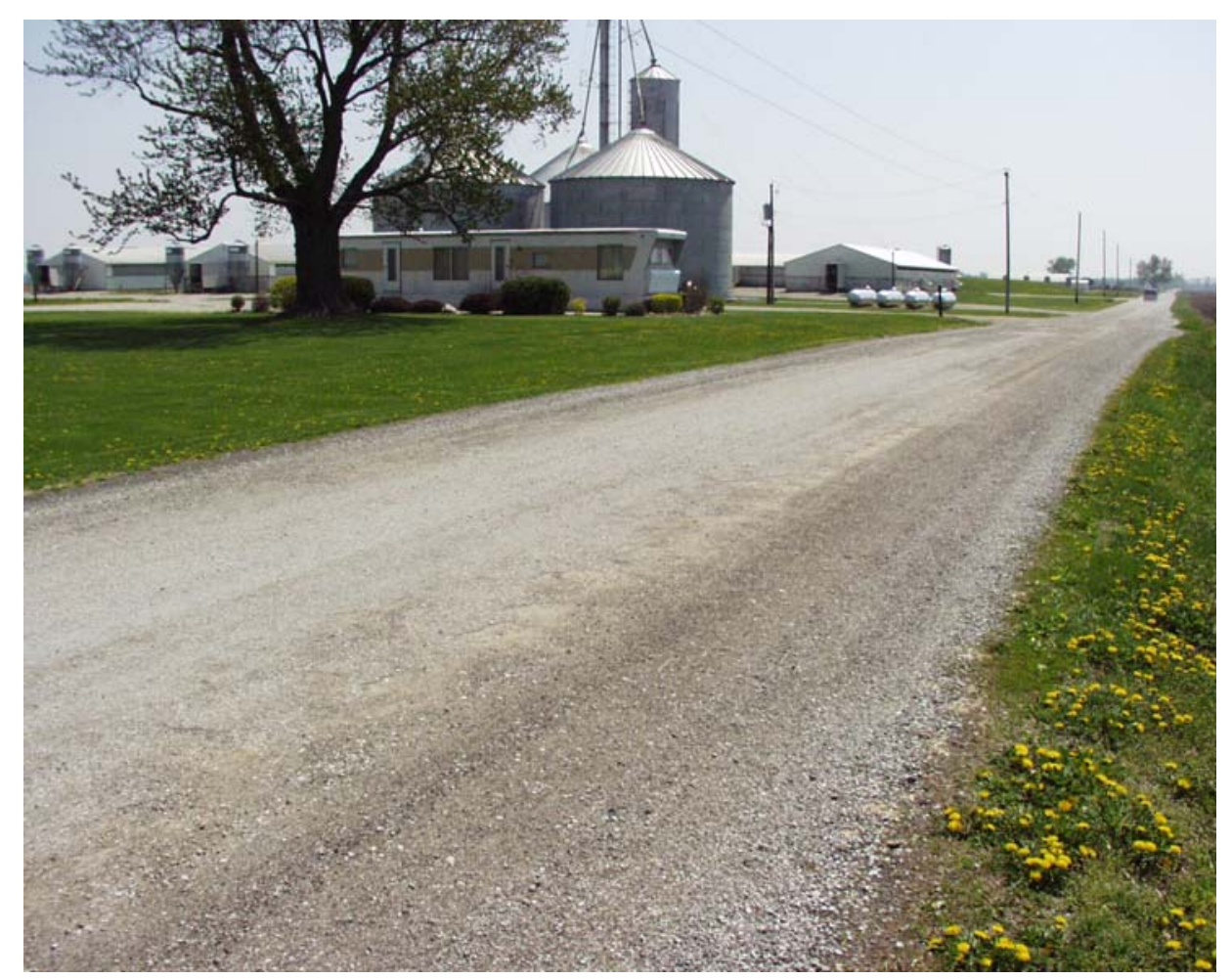

Plate 9-2 Many Paved County Roads Have Received Little or No Preservation Since Construction and are Losing Their Paved Surfaces Through Deterioration 


\subsection{IMPLEMENTATION ISSUES}

Through this study, INDOT, LTAP, and local agencies have been provided the following study products for safety investment decision-support purposes:

- A list of influential factors that affect crash occurrence at rural two-lane roads in Indiana

- Updated crash reduction factors for geometric improvements for rural two-lane state highways and new crash reduction factors for county roads in Indiana

- A systematic and objective methodology for evaluating the network-level impacts of alternative safety funding levels

- Requisite data with which an agency can carry out short-term project-level trade-off analysis between competing investments at a given location. For example, given a certain budget for a geometrically deficient road section, should the agency widen the lane, widen the shoulder, or pave the shoulder?

- Information that will help the agencies to carry out better allocation of safety enhancing resources, and to establish more effective accident risk management procedures

Implementation of the study would involve application of the above products for safety management at both state and local level, to make decisions that will, in a cost-effective manner, reduce highway crashes with secondary benefits of increased mobility, enhanced geriatric driving, reduced tort liability, and enhanced economic productivity. Given the increasing usage of rural highways, even a marginal impact of such benefits can be significant in terms of the number and/or value of fatal, injury and property damage crashes that will saved.

This study focuses on engineering solutions, a critical aspect of implementation is for INDOT and LTAP to continue supporting non-engineering solutions to the two-lane highway safety problem. It is true that engineering solutions help to offset, to some extent, the safety risks associated with the non-engineering factors. However, the need for non-engineering solutions cannot be ignored. Non-engineering implementation are in the form of supporting regulations, enforcement and public education for enhanced vehicle worthiness and safety features, sober and responsible driving, and reasonable speed limit policies, to name a few. By including such non-engineering measures in the study implementation, it is expected the maximum benefit would be realized from implementing the engineering solutions proposed by the present study.

Finally, as INDOT and LTAP set out to implement the study results, the issue of funding must be given due consideration. Given the large amount of funding needed to fix the geometric deficiencies on the state's rural two-lane system vis-à-vis budgetary restrictions currently faced at all 
levels of government, there is an urgent need for INDOT and LTAP to investigate how to (i) maximize the use of funding that is currently available through the use of innovative contracting systems and improved materials, (ii) increase funding levels from traditional sources such as the fuel tax through voter-approved increases in tax rates, and (iii) identify and tap funding sources that are alternatives to fuel tax. Various recent and ongoing research by the JTRP are addressing these issues, and the state and local agencies may be well served if the recommendations from those studies are carefully studied for possible future implementation.

\subsection{FUTURE RESEARCH}

As the state moves towards commissioning of its highway safety management system and as LTAP moves towards development of a county road inventory and safety database, a plethora of data is expected to become available to enable further detailed investigations of crash causes, and costs and effectiveness of safety projects. Future research in this area could examine the homeostasic effect of safety improvements. Also, surveys of regular rural two-lane highway and county road users could be carried out to obtain their perspectives on the safety situation.

Another area that could benefit from future research is the cost of safety improvements. The costs used in the present study came from a variety of sources such as past INDOT sponsored research, national studies and anecdotal sources. Ongoing INDOT research and possible future LTAP research may be expected to yield more reliable unit costs of safety work so that more accurate estimates of cost-effectiveness and needs assessments can be carried out.

In its analytical framework, the current study duly recognizes that a unit change in engineering factors has different effects (in absolute terms) for different existing values of the factor. For example, extending an existing 3-ft shoulder by $1 \mathrm{ft}$ may yield a different number of crash reduction compared to the extension of an existing 1 - $\mathrm{ft}$ shoulder by $1 \mathrm{ft}$, because the crash reduction factor even though it is same for each situation, is a percentage of the existing crash frequency. As such, an existing narrow shoulder which has an already large number of crashes would stand to gain a higher reduction of crashes than an already wide shoulder even though the percentage reductions are the same. In future research, this issue could be further investigated using various mathematical forms of the crash prediction question. The use of variables raised to a power other than 1.00 and interaction terms between certain factors, could be investigated.

Also, future research could involve the collection and analysis of data from Indiana or elsewhere to investigate the effectiveness and cost-effectiveness of various countermeasures such as rumble striping at the centerlines of rural two-lane roads, improvements in roadside hazard ratings in 
general, and clearance of roadside poles fences and other obstructions. Furthermore, when crash data from the entire county road network becomes available in a format that is easily amenable for integration with county road inventory and traffic databases, crash prediction models and crash reduction factors may be developed for the entire county road network or categorized families thereof, instead of the relatively parochial sample for county roads that was used in the present study. 


\section{REFERENCES}

AASHTO. [1984]. A Guide for Erecting Mailboxes on Highways. American Association of State Highway and Transportation Officials, Washington, D.C.

AASHTO. [1986]. Policy of Geometric Design of Highways and Streets. American Association of State Highway and Transportation Officials, Washington, D.C.

AASHTO. [1996]. Road Design Guide. American Association of State Highway and Transportation Officials, Washington, D.C.

Abbess, C., D. Jarret and C. C. Wright 1981]. "Accidents at Blackspots: Estimating the Effectiveness of Remedial Treatment, With Special Reference to the 'Regression-To-The-Mean' Effect". Traffic Engineering and Control, Vol. 22, No. 10, pp. 535-542.

Abdel-Aty, M.A. and Radwan, A.E. [2000] "Modeling Traffic Accident Occurrence and Involvement", Accident Analysis and Prevention, Vol 32, pp: 633-642.

Bowie, N. N., and Walz, M. [1994]. "Data Analysis of Speed Related Crash Issue." Auto and Traffic Safety, Vol. 1, No. 2, Winter, NHTSA, U.S. Department of Transportation, pp. 31-38.

Brinkman, C. and Perchonok, K. [1979]. "Hazardous Effects of Highway Features and Roadside Objects Highlights”. Public Roads. Federal Highway Administration, Washington, D.C.

Brown, D. B., Bulfin, R., and Deason. [1990]. W. Allocating Highway Safety Funds, Transportation Research Record 1270, Transportation Research Board, National Research Council, Washington, D.C.

Brown, H, Labi, S., Tarko, A. and Fricker, J. [2000]. A Tool for Evaluating Access Control on High Speed Urban Arterials, Final Report No. FHWA/IN/JTRP-98-7 Part 1: Research Report, Purdue Univeristy, West Lafayette, IN.

CATS [2003]. Indiana 2000 Crash Facts. Center for the Advancement of Transportation Safety, Purdue University, West Lafayette, IN.

Chaudoin, J.H. and Nelson, G. [1985]. Interstate Routes 15 and 40 Shoulder Rumble Strips, Report No. Caltrans-08-85-1, California DOT, CA.

Council, F. M. and Stewart J.R. [1999]. "Safety Effects of the conversion of Rural Two-Lane to Four-lane Roadways Based on Cross-Sectional Models", Paper presented at the $78^{\text {th }}$ Annual Meeting of the Transportation Research Board, Washington, D.C.

Fambro, D.B. Fitzpatrick, K. and Koppa, R. [1997]. "Determination of Stopping Sight Distances”, NCHRP Report 400. Transportation Research Board, Washington, D.C. 
Fambro, D.B., Urbanik II, T., Hinshaw, W.M., and Hanks, J.W., [1989]. Stopping Sight Distance Considerations at Crest Vertical Curves on Rural Two-lane Highway in Texas. TTI/TxDOT Report 1125-1F, Texas Transportation Institute, College Station, TX.

Farooq, O., James, D., Sinha, K. C., and. Jiang, Y. [1994].The Development of a Highway Safety Management System for Indiana: Phase I, FHWA/IN/JHRP-94/14, Joint Highway Research Project.

FHWA [1081]. Highway Safety Improvement Program (HSIP). Report Number FHWA-TS-81-218, Federal Highway Administration U.S. Department of Transportation, Washington, D.C.

FHWA [1986]. Roadside Improvements for Local Roads and Streets, Federal Highway Administration, U.S. Department of Transportation, Washington, D.C.

Federal Highway Administration (FHWA). [1995], "Travel Speeds Enforcement Efforts, and SpeedRelated Highway Statistics". FHWA-SA-95-051. U.S. Department of Transportation, Washington, D.C.

FHWA. [1997]. Rumble Strips: A Sound Investment, FHWA Publication No. FHWA-SA-017. Federal Highway Administration, Washington, D.C.

FHWA [2000]. Safety. FHWA-SA-00-051. Federal Highway Administration, U.S. Department of Transportation, Washington, D.C.

Fink, K.L. and Krammes, R.A. [1995]. "Tangent Length and Sight Distance Effects on Accident Rates at Horizontal Curves on Rural Two-lane Highways", Transportation Research Record 1500, Transportation Research Board, Washington, D.C.

Fridstorm, L. and Ingebrigsten, S. [1991] "An Aggregate Accident Model Based on Pooled, Regional Time-Series Data", Accident Analysis and Prevention, Vol. 23, pp 363-378.

Garder, P. [1995]. "Rumble Strips or Not Along Wide Shoulders Designated for Bicycle Traffic", Transportation Research Record 1502, Transportation Research Board, Washington, D.C.

Giraud, T, Choivichien, V., Labi, S. and Sinha, K.C. [2003]. Development of a Risk Management System for the Indiana Department of Transportation, Draft Final Report No. FHWA/IN/JTRP2003/20, Purdue University, W. Lafayette IN.

Glennon, J., Newman, T. and Leisch, J. [1985]. Safety and Operational Considerations for Design of Rural Highway Curves, Report No. FHWA/RD-86-035, Federal Highway Administration, Washington, D.C.

Glennon, J. [1985]. "Effect of Alignment on Highway Safety", "Relationship between Safety and Key Highway Features. State of the Art Report 6, Transportation Research Board, Washington, D.C., pp. 48-63.

Glennon, J.C. [1987]. "Effect of Pavement/Shoulder Drop-offs on Highway Safety", in State of the Art Report Number 6: Relationship between Safety and Key Highway Features, pp 1-21. Transportation Research Board, Washington, D.C. 
Graham, J.L. and Harwood, D.W. [1982]. "Effectiveness of Clear Recovery Zones", NCHRP Report 247. Transportation Research Board, Washington, D.C.

Greene, W.H. [1993]. Econometric Analysis (2nd edition), MacMillan Publishing Company, NY.

Greene, W. H. [1995]. LIMDEP Version 7.0 User's Manual, Econometric Software Inc, Bellport, NY.

Griffin, L. I., and Mak, K.K. [1988]. "Benefits to be Achieved from Widening Rural, Two-lane, Farm-to-Market Roads in Texas", Paper Presented at 1988 Annual Transportation Research Board Meeting, Transportation Research Board, Washington, D.C.

Haff, L. [1998]. Local Agency Safety Management System. Washington State Department of Transportation, Tacoma, WA.

Harwood, D.W., Council, F.M., Hauer, E., Hughes, W.E., and Vogt, A. [1998]. Prediction of the Expected Safety Performance of Rural Two-lane Highways. Report No. FHWA-RD-4584-09, Federal Highway Administration, McLean, VA.

Harwood, D.W., and Hoban, C.J. [1987]. Low-Cost Methods for Improving Traffic Operations on TwoLane Roads: Informational Guide. Report No. FHWA-IP-87-2, Federal Highway Administration, Washington, D.C.

Harwood, D.W., and St. John, A.D. [1985]. Passing Lanes and Other Operational Improvements on Twolane Highways. Report No. FHWA-RD-85-028, Federal Highway Administration, Washington, D.C.

Harwood, D. W. [1993]. Use of Rumble Strips to Enhance Safety. NCHRP Report 486, Transportation Research Board, Washington, D.C.

Harwood, D. W., Kohlman Rabbani, E.R., Richard, K.R., McGee, H.W., and Gittings, G.L. [2003]. Systemwide Impact of Safety and Traffic Operations Design Decisions of Resurfacing, Restoration, or Rehabilitation (RRR) Projects. NCHRP Report 486, Transportation Research Board, Washington, D.C.

Hauer, E. [1992]. Empirical Bayes Approach to Estimation of Unsafety: The Multivariate Regression Approach. Accident Analysis and Prevention, Vol. 24, No. 5, pp. 456-478.

Hauer, E. [2000(1)].” Lane Width and Safety". Reflections of Personal Opinions, Unpublished. Source: Authors personal internet website.

Hauer, E. [2000(2)]. "Shoulder Width, Shoulder Paving and Safety". Reflections of Personal Opinions, Unpublished. Source: Authors personal internet website.

Hauer, E., D. W. Harwood and M. S. Griffith. [2002]. "Estimating Safety by the Empirical Bayes Method: A Tutorial", Transportation Research Record No. 1784, National Research Council, Transportation Research Board, Washington, D. C. pp. 126-131. 
Hickey, J.J. [1997]. "Shoulder Rumble Strip Effectiveness", Transportation Research Record 1573, Transportation Research Board, Washington, D.C.

Higle, J. L., and Witkowski, J.M. [1988]. "Bayesian Identification of Hazardous Locations", Transportation Research Record 1185, Transportation Research Board, National Research Council Washington, D.C. pp. 24-36.

Hughes, W.E., Saremi, A.R. and Paniati, J.F. [1996]. "Vehicle-Animal Crashes: An Increasing Safety Problem", ITE Journal August 1996 Edition, ITE, Washington, D.C.

IIHS [1982]. 1985 -1986 Study on Deer Collisions, Insurance Institute for Highway Safety, Arlington, VA.

INDOT [2000]. Design Manual - Part V: Road Design, Volumes I \& II,. Design and Construction Division, Indiana Department of Transportation Indianapolis, IN.

INDOT [2003]. Indiana Safety Management System Database, Safety Management Unit, Program Development Division, Indiana Department of Transportation, Indianapolis, IN.

Jones, B., Janssen, L., and Mannering, F. [1991]. "Analysis of the Frequency and Duration of Frequency Accidents in Seattle", Accident Analysis and Prevention, Vol. 23, No. 4, pp. 239-255.

Jovanis, P.P., and Chang, H. L. [1987]. "Modeling the Relationship of Accidents to Miles Traveled", Transportation Research Record 1068, Transportation Research Board, National Research Council, Washington, DC, pp. 42-51.

Kaji, T. and K., C. Sinha. [1980]. A Cost Effectiveness Approach for the Evaluation of Highway Safety Improvement in the State of Indiana. JHRP-80-11, Joint Transportation Research Program, Purdue University, West Lafayette, IN.

Karlaftis, M.G. and Tarko, A. P. [1998]. "Heterogeneity Considerations in Accident Modeling", Accident Analysis and Prevention, Vol. 30, pp: 425-433.

Labi, S. [2001]. "Impact Evaluation of Highway Pavement Maintenance Activities", Ph.D. Dissertation, Purdue University, W. Lafayette, IN.

Lalani, N. (Chair) [1998]. Access Management - A Key to Safety and Mobility. An ITE Informational Report Brochure, Publication No. IR-095.

Lamptey, G., Labi, S., and Sinha, K.C. [2004]. “A Proposed Framework for Safety Investment Planning for Indiana", scheduled for presentation at 83 $3^{\text {rd }}$ Annual Meeting of the Transportation Research Board, National Research Council, Washington, D.C.

Ligion, C.M., Carter, E.C., Joost D.B., Wolman, W.F. [1985]. Effects of Shoulder Textured Treatments on Safety. Report No. FHWA-RD-85/027, Federal Highway Administration, McLean, VA. 
Ludwig, J., and Brickmeyer T. [1983]. "Evaluation of 2.4m Fences and one-way Gates for Reducing Deer-Vehicle Collisions in Minnesota", Transportation Research Record 913, Transportation Research Board, Washington, D.C.

Mak, K. [1995]. "Methods for Analyzing the Cost-Effectiveness of Roadside Safety Improvements". Transportation Research Circular, Number 435: Roadside Safety Issues. Transportation Research Board, Washington, D.C.

McCree, J. [1988]. "Low-Cost Road Improvements", Illinois Municipal Review, April 1988 Issue, Illinois Municipal League. Springfield, IL, pp. 19-20.

McGuigan, D. R. D. [1981]. "The Use of Relationships between Road Accidents and Traffic Flow in 'Blackspot' Identification”, Traffic Engineering and Control, pp. 448-453

Miaou, S.P. [1998]. "Vertical Grade Analysis Summary", unpublished Technical Memorandum to FHWA, Washington, D.C.

Miaou, S. P., P. S. Hu, T. Wright, S. C. Davis and A. K. Rathi. [1993]. Development of Relationship between Truck Accidents and Geometric Design: Phase I. Report No. FHWA-RD-91-124, Federal Highway Administration, Washington, D.C.

Milton, J. and Mannering, L. [1998]. " The Relationship Among Highway Geometrics, TrafficRelated Elements and Motor Vehicle Accident Frequencies”, Transportation, Vol. 25, pp 395413.

Nelson, P. [1994]. "Deer Watch", National Wildlife, Vol. 32, No. 6. National Wildlife Federation, Washington D.C.

Ogden, K.W. [1997]. "The Effects of Paved Shoulders on Accidents on Rural Highways", Accident Analysis and Prevention 29 (3) pp. 353-362.

Olson, P.L., Cleveland, D.E., Fancher, P.S., Kostiniuk, L.P. and Schneider, L.W. [1884]. "Parameters Affecting Stopping Sight Distance", NCHRP Report 270. Transportation Research Board, Washington, D.C.

Pal, R., and Sinha, K. C. [1998]. Optimization Approach To Highway Safety Improvement Programming, Transportation Research Record 1640, Transportation Research Board, National Research Council, Washington, D.C.

Pasupathy, R.K., Ivan, J.N. [2000]. "Single and Multi-vehicle Crash Prediction Models for TwoLane Roadways", Final Report, New England Universities Transportation Center, Report Number NEUTC UCNR9-8, Cambridge, MA.

Perchonok, K., Ranney, T., Baum, S., Morris, D., and Eppich, K. [1978]. Hazardous Effects of Highway Features and Roadside Objects, Volume I: Literature Review and Methodology, and Volume II: Findings. Report No. FHWA-RD-78/201 and 202, Federal Highway Administration, McLean, VA. 
Persaud, B., C. Lyon, and T. Nguyen. [1999]. Empirical Bayes Procedure for Ranking Sites for Safety Investigation by Potential for Safety Improvement., Transportation Research Record 1665, Transportation Research Board, National Research Council, Washington, D.C., 1999, pp. 7-12

Retting, R. [2003]. "Center Line Rumble Strips Reduce Crash Risk on Rural Two-Lane Roads", The Pothole Gazette Vol. 21 No. 3. Indiana Local Technical Assistance Program (LTAP). West Lafayette, IN.

Rinde, E [1977]. Accident Rates vs. Shoulder Widths. Report No. CA-DOT-RT-3147-1-77-01, Federal Highway Administration, Washington, D.C.

Ross, H.E., Walker, K.C., and Lindsay, W.J. [1980]. The Rural Mailbox-A Little Known Hazard. Texas Transportation Institute, College Station, TX.

Schafer, J.A., Penland, S., and Carr, W.P. [1985]. Effectiveness of Wildlife Warning Reflectors in Reducing Deer-Vehicle Accidents in Washington State", Transportation Research Record 1010, Transportation Research Board, Washington, D.C.

Sinha, K. C., Kaji, T., and Liu, C. [1981]. Optimal Allocation of Funds for Highway Safety Improvement Projects, Transportation Research Record 808, Transportation Research Board, National Research Council, Washington, D.C.

Strathman, J.G., Duecker, K.J. Zhang, J., and Williams, T. [2001]. "Analysis of Design Attributes and Crashes on the Oregon Highway System." Final Report, SPR 321, Center for Urban Studies, Portland State University, Portland, OR.

St. John, A.D., and Kobett, D.R. [1978]. "Grade Effects on Traffic Flow Stability and Capacity", NCHRP Report 185. Transportation Research Board, Washington, D.C.

Stoughton, R. [1996]. An Old-timer Suggests Some Activities for Improving Roadside Safety. Transportation Research Circular, Number 453: Roadside Safety Issues Revisited. Transportation Research Board, Washington D.C.

Tarko, A., Eranky, S., and Sinha, K.C. [1998]. "Methodological Considerations in the Development and Use of Crash Reduction Factors." Paper Presented at the 77th Annual Meeting of the Transportation Research Board, Washington, D.C.

Tarko A. and DeSalle, B. [2002]. "Internet Supported Evaluation of Highway Safety", JTRP Final Report, FHWA/IN/JTRP-2002/9, Purdue University.

Tarko, A., Eranky, S., Sinha, K.C., and Scienteie, R. [1999]. Crash Reduction Factors for Improvement Projects on Road Sections in Indiana. Presented at the 78th Annual Meeting of the Transportation Research Board, Washington, D.C.

Tarko, A.P., and Sinha, K.C. and Farooq, O.A. [1997] "Methodology for Identifying Highway Safety Problem Areas", Presented in the $76^{\text {th }}$ Annual Meeting of the Transportation Research Board Meeting, Washington, D.C. 
Tessner, J. [1996] "Rural and Urban Crashes: A Comparative Analysis", National Highway Traffic Safety Administration.

Thomas, L.W. [2003]. "Tort Liability of Highway Agencies”, Selected Studies in Transportation Law Volume 4, National Cooperative Highway Research Program, Transportation Research Board, Washington, D.C.

Transportation Research Board (TRB) [1987]. Special Report 294 55: Designing Safer Roads: Practices for Resurfacing, Restoration and Rehabilitation. National Research Council, Washington, D.C.

Transportation Research Board (TRB) [1998]. Special Report 254 Managing Speed: Review of Current Practice for Setting and Enforcing Speed Limits. National Research Council, Washington D.C.

Turner, D.S. [1984]. "Prediction of Bridge Accident Rates", Journal of Transportation Engineering, Vol. 110, No. 1, American Society of Civil Engineers, New York, NY.

Turner, D.S., and Fambro, D.B. [1981]. "Effects of Paved Shoulders on Accident Rates for Rural Texas Highways", Transportation Research Record 819, Transportation Research Board, Washington, D.C.

Ullman, G.L. and Pezdolt, V.J. [1997]. Delineation of Bridges and Culverts in Texas. Report No. FHWA/TX-98/1366-1F, Federal Highway Administration, Washington D.C.

Viano, D. C., and Ridella, S. [1996]. "A Case Study of Fatal Accident Circumstances and Configurations." No. 960458. Society of Automotive Engineers, International Congress and Exposition, Detroit, MI, pp. 123-134.

Vogt, A., and J. G. Bared [1998]. Accident Models for Two-Lane Rural Roads: Segments and Intersections. Report No. FHWA-RD-98-133, Federal Highway Administration, Washington, D.C.

Ward, A.L. [1984]. "Mule-deer Behavior in Relation to Fencing and Underpasses on Interstate 80 in Wyoming", Transportation Research Record 859, Transportation Research Board, Washington D.C.

Zacks, J.L. [1986]. "Do White-tailed Deer Avoid Red? An Evaluation of the Premise Underlying the Design of Swareflex Wildlife Reflectors (Discussion and Closure)", Transportation Research Record 859, Transportation Research Board, Washington D.C.

Zador, P, Stein, H., Hall, J. and Wright, P. [1985]. Superelevation and Roadway Geometry: Deficiency at Crash Site and on Grades (Abridgement). Insurance Institute for Highway Safety, Washington, D.C.

Zegeer, C.V. and Parker, M.R. [1984]. Cost-Effectiveness of Countermeasures for Utility Pole Accidents: User Manual. Report No. FHWA-IP-84-13, Federal Highway Administration, Washington, D.C.

Zegeer, C.V. and Deacon, J.A. [1987]. "Effect of lane width, shoulder width and shoulder type on highway safety", In State of the Art Report Number 6: Relationship Between Safety and Key Highway Features, pp 1-21. Transportation Research Board, Washington, D.C. 
Zegeer, C.V., Hummer, J., Reinfurt, D., Herf, L., and Hunter, W. [1987]. Safety Effects of Cross Section Design For Two-Lane Roads- Volumes I and II. Report No. FHWA-RD-87-008, Federal Highway Administration, Washington, D.C.

Zegeer, C.V. and Reinfurt, D.W., Hummer, J., Herf, L., Hunter, W. [1988]. "Effects of Crosssection design for two-lane roads", Transportation Research Record 1195, pp 20-32. Transportation Research Board, Washington, D.C.

Zegeer, C.V., Stewart, R., Reinfurt, D., Council, F.M., Newman, T., Hamilton, E., Miller, T, and Hunter, W. [1990]. Cost-Effective Geometric Improvements for Safety Upgrading of Horizontal Curves. Report No. FHWA-RD-90-021, Federal Highway Administration, Washington D.C.

Zegeer, C.V., Stewart, J., Council, F. and Reinfurt, D. [1991]. Cost-Effective Geometric Improvements for Safety Upgrading of Horizontal Curves. Report No. FHWA-RD-90-021, Federal Highway Administration, Washington, D.C.

Zegeer, C.V., Stewart, R., Council, F. [1994]. "Roadway Widths for Low Traffic-Volume Roads", NCHRP Report 362, Transportation Research Board, Washington, D.C. 
<smiles>C1C2C[As]1C2</smiles>

APPENDICES 


\section{APPENDIX A}

\section{APPENDIC A1 Distribution of Public Road Mileage by Area Class and Jurisdiction}

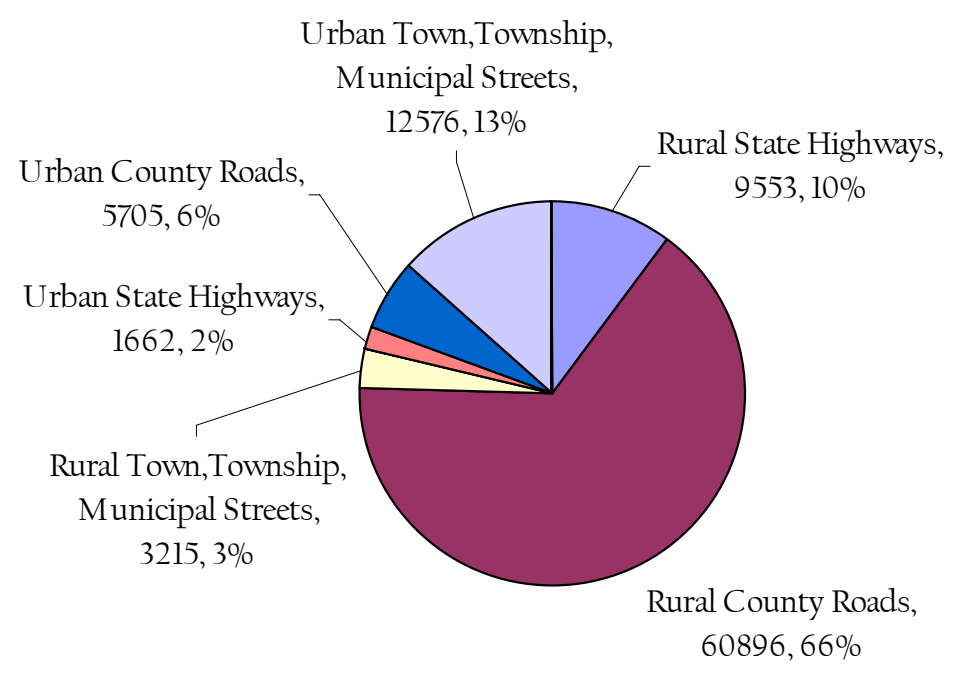

Total Public Road Inventory Size $=93,608$ miles Source: Table HM-10, Highway Statistics, US DOT

\section{APPENDIX A2: Distribution of Public Road Mileage by Functional Class}

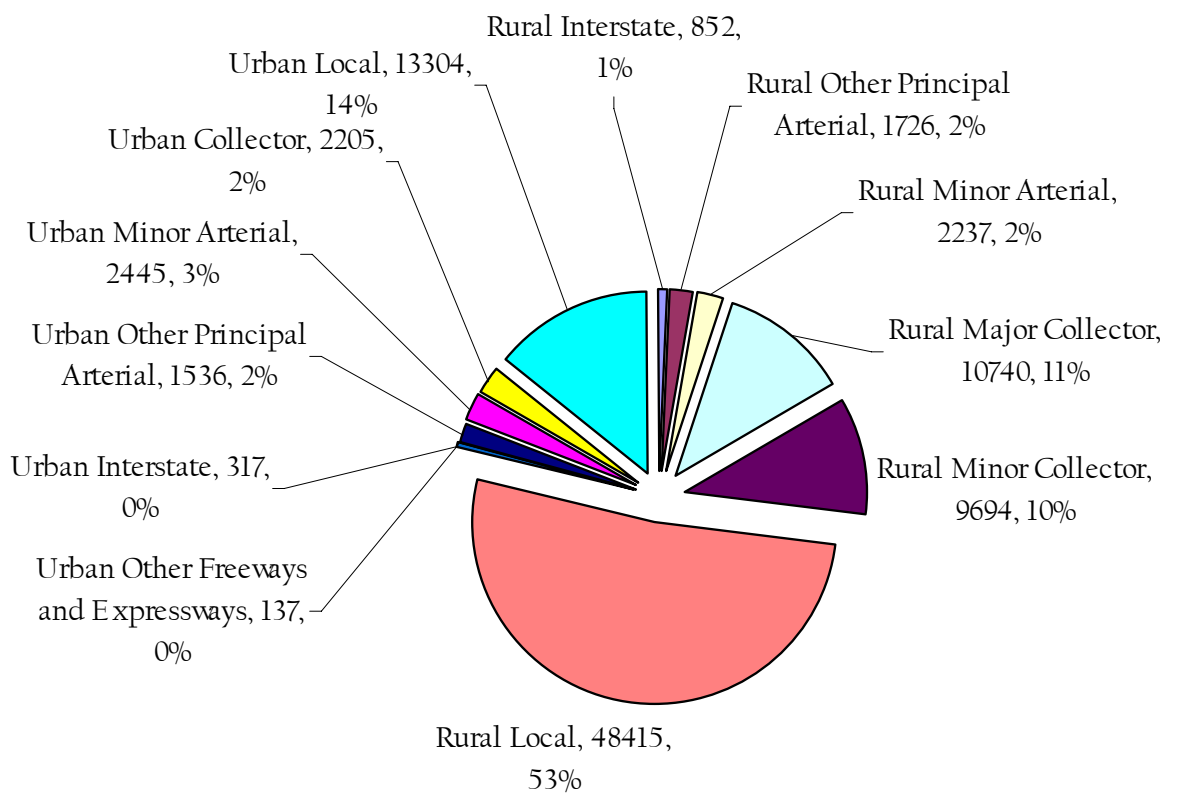

Total Public Road Inventory Size $=93,608$ miles Source: Table HM-20R, Highway Statistics, US DOT 
APPENDIX A3: Distribution of State Highway System Road Mileage by Functional Class

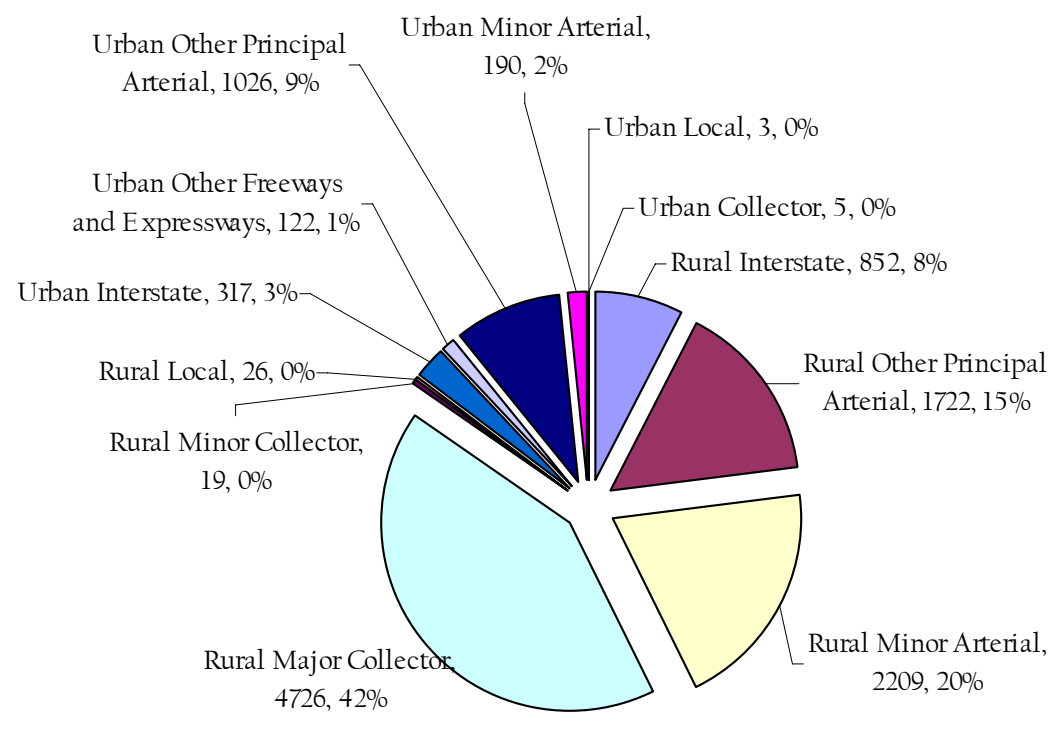

Total State Highway System Inventory Size = 11, 217 miles Source: Table HM-80R, Highway Statistics, US DOT

\section{APPENDIX A4: Distribution of Local Road System Road Mileage by Functional Class}

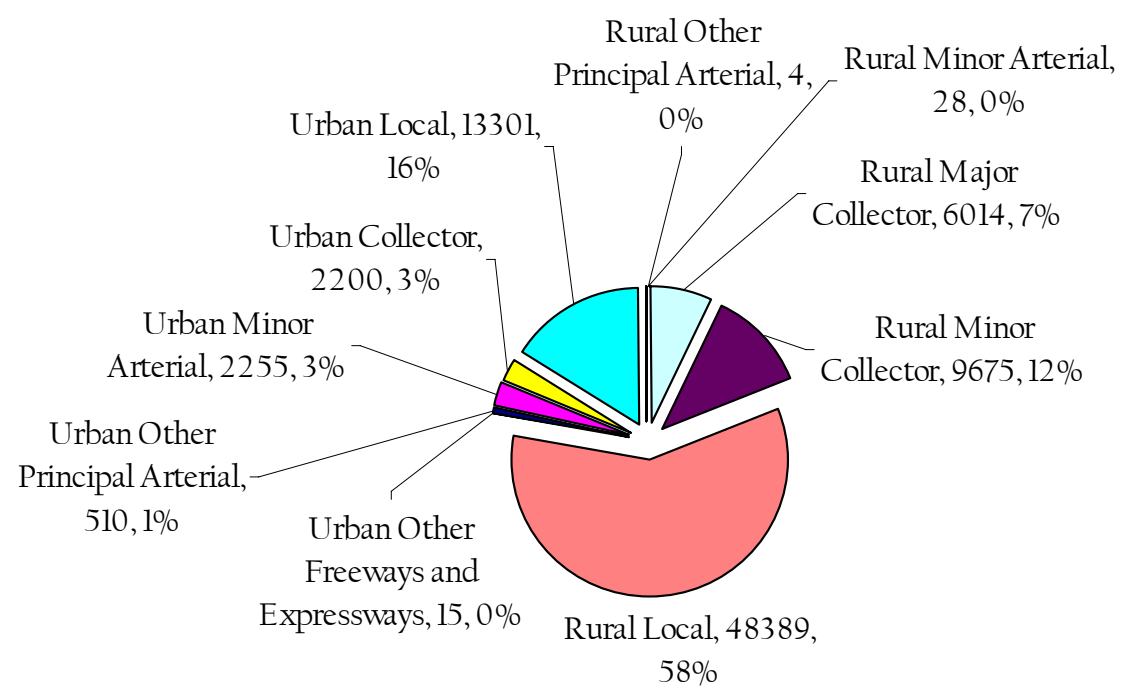

Total State Highway System Inventory Size = 82,391 miles Sources: Tables HM-20R and HM-80R, Highway Statistics, US DOT 


\section{APPENDIX A5: Distribution of Public Road VMT by Functional Class (in millions)}

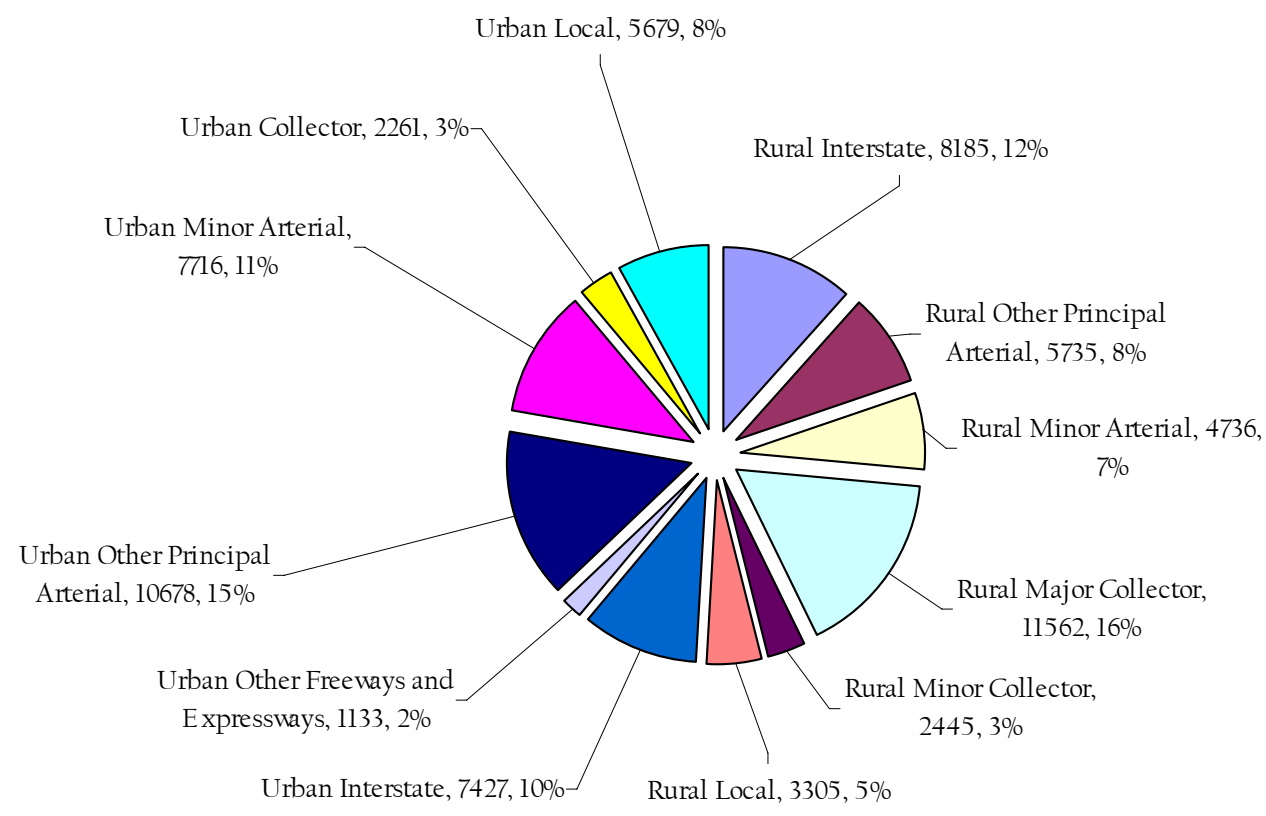

Total Public Road VMT = 71 Billion (approx.)

Source: Table VM-2R, Highway Statistics, US DOT 
APPENDIX B: NEWS CLIPPING ON RURAL ROAD SAFETY
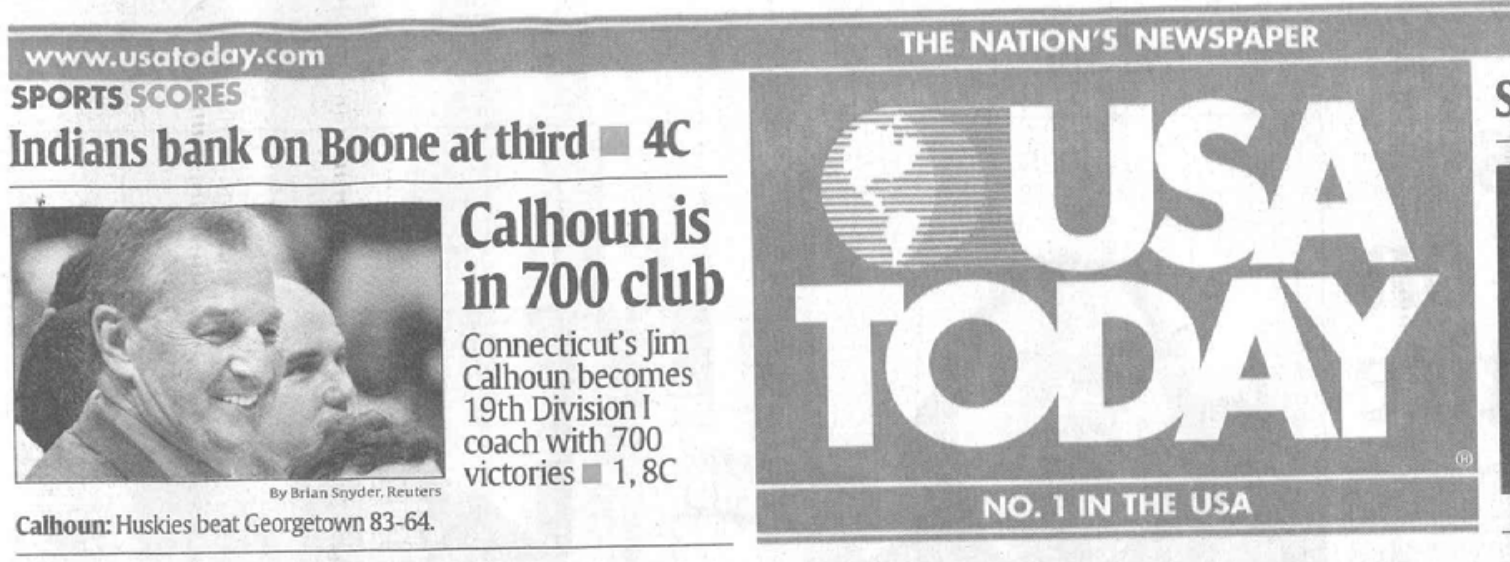

Thursday, March 3, 2005

\section{Study shows rural roads are most deadly}

By Tom Kenworthy USA TODAY

The death rate for motorists on rural roads was more than $21 / 2$ times the rate for driving on all other roads in 2003 , a study to be released today shows.

Safety improvements on rural, non-interstate routes have lagged, although driving on all U.S. roads and highways has become less dangerous since 1990 , according to an analysis of federal highway data by The Road Information Program (TRIP).

"The nation's rural roads ... are exposing rural residents and visitors to an unacceptable level of risk," says William Wil-

kins, executive director of the million miles driven, compared highway information research with 0.99 on all other roads. organization in Washington.

"We know how to make rural roads safer. What is missing is adequate funding for road safe- $32 \%$. The death rate on rural ty projects that will save numerous lives."

Among the study's findings:

- $52 \%$ of the

42,301 average

annual traffic

deaths from 1999

State-by-state statistics

nural-road death rates, $5 \mathrm{~A}$ through 2003 occurred on ru- eas are more likely than urban ral, non-interstate routes, al- roads to have features that though travel on those roads represents $28 \%$ of miles driven. $\checkmark$ The death rate on rural roads in 2003 was 2.72 per 100 same period. $\rightarrow$ Many rural areas, particularly in the West and South, are gaining population, but make driving hazardous. shoulders, sharp curves, steep slopes and pavement drop-offs. is cluding rural roads decreased
Tools to improve safety include rumble strips, better signs, lane markings and lighting, guardrails, and removal of obstacles along roadsides. A transportation spending bill pending in Congress could increase funding for rural road improvements. But substantial roads declined by $21 \%$ during the

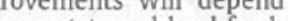
more on state and local funding, which represent about on roads and highways, says Frank Moretti, director of policy and research for TRIP.

TRIP is a non-profit group supported by insurance companies, labor unions and businesses involved in highway construction and engineering. three-quarters of U.S. spending

Dangero

States with rates in 200 million mile:

数 Rural, ne roads iv All other Ariz.

Fla S.C. 0. Mont.

Ky. 


\section{APPENDIX C}

\section{Cl- QUESTIONNAIRE SURVEY SOLICITATION LETTER}

To

Dear Sir,

We are writing to request your help in obtaining important information about your experiences with respect to two-lane rural highway crash locations in your county. The purpose of the attached questionnaire is to gather data and statistics that the Center for Advancement of Transportation Safety (CATS), Purdue University, will use to identify high-risk areas with respect to crashes and to analyze the impact in your county.

This brief questionnaire requires approximately 5 minutes completing it. Please send the questionnaire back in the enclosed envelope. Your participation will assist us in developing policies and programs to increase safety on Rural Two-Lane Roads in Indiana.

The success of CATS effort to create and advocate policies that promote safety depends largely on the quality of the information we receive about your experiences. Your participation in this effort will ensure that CATS obtains sufficient information to develop various policy recommendations and programs.

If you have any questions and comments, please contact:

Jose E. Thomaz

CATS, Potter Engineering Building,

Purdue University

West Lafayette IN, 47907

Phone - 765

weekdays between 8:30 a.m. and 5:00 p.m. Central standard time. You may call or email us at jthomaz@ecn.purdue.edu

Thank you in advance for your assistance with this valuable undertaking. It will be nice if you could respond at the earliest.

Sincerely,

Jose E. Thomaz

Principal Investigator

And

Sravanthi Konduri

Research Assistant 


\section{C2- COPY OF QUESTIONNAIRE SURVEY FORM}

\section{QUESTIONNAIRE}

Roads of interest: Only Two-lane, Rural roads, which are not intersections.

In the following table please indicate the road segments that you consider as dangerous locations with respect to crashes in your county. Please note that Crash locations within 200 feet distance of intersections are not included.

Also please indicate any local names (other than the designated names) of any of these roads.

\begin{tabular}{|l|l|l|}
\hline NAME OF THE ROAD & OTHER NAMES & $\begin{array}{c}\text { Please indicate directions to help us locate } \\
\text { (crash related sites) }\end{array}$ \\
\hline & 1 & \\
& 2 & \\
& 3 & \\
& 4 & \\
\hline & 1 & \\
& 2 & \\
& 3 & \\
& 4 & \\
\hline & 1 & \\
& 2 & \\
& 3 & \\
& 4 & \\
& 1 & \\
& 2 & \\
& 3 & \\
& 4 & \\
\hline & 1 & \\
& 2 & \\
& 3 & \\
\hline
\end{tabular}

A county map is also attached if you would like to indicate the dangerous locations on the map, in place of, or in addition to the above table. Please mark the routes for the county roads if needed.

Thank you very much for your valuable time and cooperation. 


\section{APPENDIX D CRASH PREDICTION MODELING RESULTS}

Functional forms for the models are shown in Equations 4-1 (Chapter 4)

Table D-1: Injury Crash Model for Rural Major Collectors

\begin{tabular}{|c|c|c|c|c|}
\hline & Coefficient & Std.Error. & t-ratio & P-value \\
\hline Constant Term (ONE) & -6.80007 & 0.379207 & -17.9323 & 6.44E-14 \\
\hline Section Length (L_LENG) & 0.936676 & 0.0733411 & 12.7715 & 6.44E-14 \\
\hline Section Traffic Volume (L_AADT) & 0.8705 & 0.0481 & 18.0932 & 0.0000 \\
\hline Lane Width (LW) & -0.0968 & 0.0292 & -3.3145 & 0.0009 \\
\hline Shoulder Width (SW) & -0.0299 & 0.0143 & -2.0901 & 0.0366 \\
\hline Pavement Surface Friction (FR) & -0.0164 & 0.0091 & -1.8029 & 0.0714 \\
\hline Horizontal Curvature (ARAD) & -0.0268 & 0.0135 & -1.9912 & 0.0465 \\
\hline Vertical Grade (AGRAD) & 0.0553 & 0.0205 & 2.7029 & 0.0069 \\
\hline Alpha & 0.2414 & 0.0347 & 6.9634 & 0.0000 \\
\hline $\begin{array}{lc}\text { Number of observations } & = \\
\text { Log likelihood function } & = \\
\text { Restricted log likelihood } & = \\
\text { Chi-squared } & = \\
\text { Response variable is the Natural Log o }\end{array}$ & $\begin{array}{l}1332 \\
-2064.036 \\
-2114.520 \\
100.967[.0000] \\
\text { Jumber of Crashes }\end{array}$ & & & \\
\hline
\end{tabular}

Table D-2: PDO Crash Model for Rural Major Collectors

\begin{tabular}{|c|c|c|c|c|}
\hline & Coefficient & Std.Error. & t-ratio & P-value \\
\hline ONE Constant Term (ONE) & -4.06689 & 0.261934 & -15.5264 & 6.44E-14 \\
\hline Section Length (L_LENG) & 0.8706 & 0.0454 & 19.1745 & 0.0000 \\
\hline Section Traffic Volume (L_AADT) & 0.6259 & 0.0358 & 17.5072 & 0.0000 \\
\hline Lane Width (LW) & -0.0617 & 0.0200 & -3.0825 & 0.0021 \\
\hline Shoulder Width (SW) & -0.0119 & 0.0106 & -1.1242 & 0.2609 \\
\hline Pavement Surface Friction (FR) & -0.0190 & 0.0071 & -2.6765 & 0.0074 \\
\hline Horizontal Curvature (ARAD) & -0.0163 & 0.0102 & -1.6003 & 0.1095 \\
\hline Vertical Grade (AGRAD) & 0.1100 & 0.0153 & 7.1801 & 0.0000 \\
\hline Alpha & 0.2991 & 0.0207 & 14.4834 & 0.0000 \\
\hline $\begin{array}{lr}\text { Number of observations } & = \\
\text { Log likelihood function } & = \\
\text { Restricted log likelihood } & = \\
\text { Chi-squared } & = \\
\text { Response variable is the Natural Log o }\end{array}$ & $\begin{array}{l}1332 \\
-3957.622 \\
-5243.300 \\
2571.356[.0 \\
\text { Number of } \mathrm{Cr}_{1}\end{array}$ & & & \\
\hline
\end{tabular}


Table D-3: Fatal+Injury Crash Model for Rural Major Collectors

\begin{tabular}{|c|c|c|c|c|}
\hline & Coefficient & Std.Error. & t-ratio & P-value \\
\hline Constant Term (ONE) & -6.6231 & 0.3657 & -18.1090 & 0.0000 \\
\hline Section Length (L_LENG) & 0.9237 & 0.0701 & 13.1796 & 0.0000 \\
\hline Section Traffic Volume (L_AADT) & 0.8526 & 0.0471 & 18.1155 & 0.0000 \\
\hline Lane Width (LW) & -0.0928 & 0.0286 & -3.2473 & 0.0012 \\
\hline Shoulder Width (SW) & -0.0321 & 0.0141 & -2.2752 & 0.0229 \\
\hline Pavement Surface Friction (FR) & -0.0156 & 0.0089 & -1.7547 & 0.0793 \\
\hline Horizontal Curvature (ARAD) & -0.0262 & 0.0132 & -1.9820 & 0.0475 \\
\hline Vertical Grade (AGRAD) & 0.0541 & 0.0200 & 2.7098 & 0.0067 \\
\hline Alpha & 0.239972 & 0.0335338 & 7.15611 & $8.36 \mathrm{E}-13$ \\
\hline $\begin{array}{ll}\text { Number of observations } & = \\
\text { Log likelihood function } & = \\
\text { Restricted log likelihood } & = \\
\text { Chi-squared } & = \\
\text { Response variable is the Number of C }\end{array}$ & $\begin{array}{l}1332 \\
-2111.145 \\
-2164.666 \\
107.043[.0000]\end{array}$ & & & \\
\hline
\end{tabular}

Table D-4: "Total Crashes" Model for Rural Major Collectors

\begin{tabular}{|c|c|c|c|c|}
\hline & Coefficient & Std.Error. & t-ratio & P-value \\
\hline Constant Term (ONE) & -4.1055 & 0.2392 & -17.1646 & 0.0000 \\
\hline Section Length (L_LENG) & 0.8928 & 0.0419 & 21.3234 & 0.0000 \\
\hline Section Traffic Volume (L_AADT) & 0.6749 & 0.0319 & 21.1653 & 0.0000 \\
\hline Lane Width (LW) & -0.0680 & 0.0184 & -3.6983 & 0.0002 \\
\hline Shoulder Width (SW) & -0.0178 & 0.0096 & -1.8648 & 0.0622 \\
\hline Pavement Surface Friction (FR) & -0.0186 & 0.0065 & -2.8871 & 0.0039 \\
\hline Horizontal Curvature (ARAD) & -0.0184 & 0.0093 & -1.9904 & 0.0465 \\
\hline Vertical Grade (AGRAD) & 0.0964838 & 0.0138938 & 6.94438 & $3.83 \mathrm{E}-12$ \\
\hline Alpha & 0.257655 & 0.0165636 & 15.5555 & $6.44 \mathrm{E}-14$ \\
\hline $\begin{array}{ll}\text { Number of observations } & = \\
\text { Log likelihood function } & = \\
\text { Restricted log likelihood } & = \\
\text { Chi-squared } & = \\
\text { Response variable is the Natural Log o }\end{array}$ & $\begin{array}{r}1332 \\
-4267.163 \\
-6046.508 \\
3558.689 \\
\text { Number of }\end{array}$ & & & \\
\hline
\end{tabular}


Table D-5: Injury Crash Model for Rural Minor Arterials

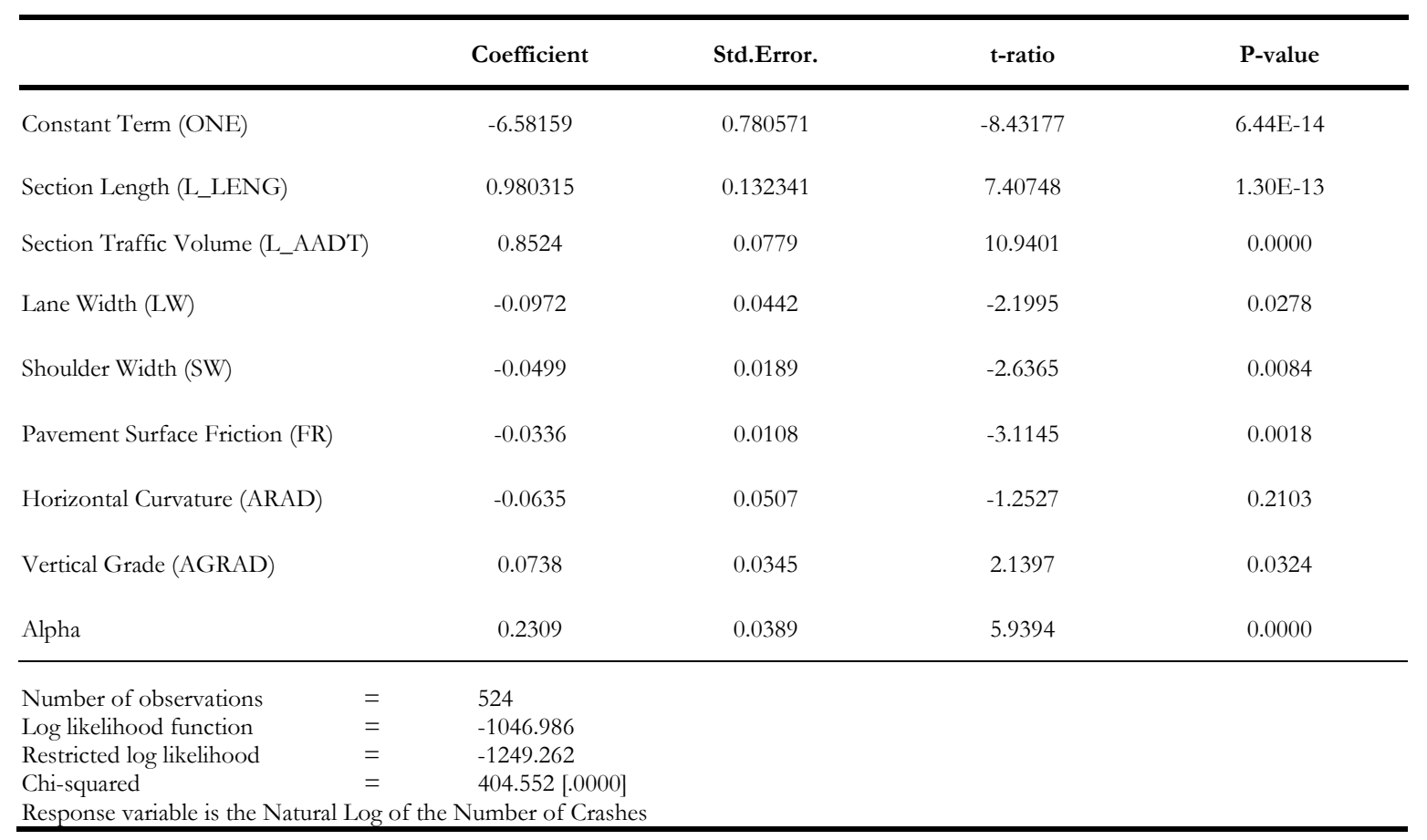

Table D-6: PDO Crash Model for Rural Minor Arterials

\begin{tabular}{|c|c|c|c|c|}
\hline & Coefficient & Std.Error. & t-ratio & P-value \\
\hline Constant Term (ONE) & -3.81056 & 0.582918 & -6.53704 & $6.31 \mathrm{E}-11$ \\
\hline Section Length (L_LENG) & 0.9981 & 0.0831 & 12.0165 & 0.0000 \\
\hline Section Traffic Volume (L_AADT) & 0.6363 & 0.0609 & 10.4564 & 0.0000 \\
\hline Lane Width (LW) & -0.0960 & 0.0346 & -2.7781 & 0.0055 \\
\hline Shoulder Width (SW) & -0.0317 & 0.0146 & -2.1756 & 0.0296 \\
\hline Pavement Surface Friction (FR) & -0.0127 & 0.0086 & -1.4877 & 0.1368 \\
\hline Horizontal Curvature (ARAD) & -0.0722 & 0.0354 & -2.0391 & 0.0414 \\
\hline Vertical Grade (AGRAD) & 0.0944 & 0.0255 & 3.7029 & 0.0002 \\
\hline Alpha & 0.2289 & 0.0228 & 10.0254 & 0.0000 \\
\hline $\begin{array}{lr}\text { Number of observations } & = \\
\text { Log likelihood function } & = \\
\text { Restricted log likelihood } & = \\
\text { Chi-squared } & = \\
\text { Response variable is the Natural Log }\end{array}$ & $\begin{array}{l}524 \\
-1522.784 \\
-11756.594 \\
467.621[.0 \\
\text { e Number of } \mathrm{C}\end{array}$ & & & \\
\hline
\end{tabular}


Table D-7: Fatal+Injury Crash Model for Rural Minor Arterials

\begin{tabular}{|c|c|c|c|c|}
\hline & Coefficient & Std.Error. & t-ratio & $\mathbf{P}$-value \\
\hline Constant Term (ONE) & -6.4612 & 0.7645 & -8.4517 & 0.0000 \\
\hline Section Length (L_LENG) & 0.9964 & 0.1288 & 7.7362 & 0.0000 \\
\hline Section Traffic Volume (L_AADT) & 0.8255 & 0.0769 & 10.7402 & 0.0000 \\
\hline Lane Width (LW) & -0.0896 & 0.0431 & -2.0813 & 0.0374 \\
\hline Shoulder Width (SW) & -0.0511 & 0.0188 & -2.7149 & 0.0066 \\
\hline Pavement Surface Friction (FR) & -0.0330 & 0.0108 & -3.0580 & 0.0022 \\
\hline Horizontal Curvature (ARAD) & -0.0580 & 0.0467 & -1.2413 & 0.2145 \\
\hline Vertical Grade (AGRAD) & 0.0866 & 0.0336 & 2.5802 & 0.0099 \\
\hline Alpha & 0.232416 & 0.0383815 & 6.05541 & $1.41 \mathrm{E}-09$ \\
\hline $\begin{array}{lr}\text { Number of observations } & = \\
\text { Log likelihood function } & = \\
\text { Restricted log likelihood } & = \\
\text { Chi-squared } & = \\
\text { Response variable is the Natural Log }\end{array}$ & $\begin{aligned} & 524 \\
&-1028.653 \\
&-1071.173 \\
& 85.040[.0 \\
& \text { e Number of }\end{aligned}$ & & & \\
\hline
\end{tabular}

Table D-8: “Total Crashes” Model for Rural Minor Arterials

\begin{tabular}{|c|c|c|c|c|}
\hline & Coefficient & Std.Error. & t-ratio & P-value \\
\hline Constant Term (ONE) & -3.9100 & 0.5574 & -7.0143 & 0.0000 \\
\hline Section Length (L_LENG) & 0.9995 & 0.0811 & 12.3222 & 0.0000 \\
\hline Section Traffic Volume (L_AADT) & 0.6834 & 0.0562 & 12.1497 & 0.0000 \\
\hline Lane Width (LW) & -0.0930 & 0.0317 & -2.9342 & 0.0033 \\
\hline Shoulder Width (SW) & -0.0391 & 0.0135 & -2.8908 & 0.0038 \\
\hline Pavement Surface Friction (FR) & -0.0161 & 0.0080 & -2.0064 & 0.0448 \\
\hline Horizontal Curvature (ARAD) & -0.0742 & 0.0314 & -2.3614 & 0.0182 \\
\hline Vertical Grade (AGRAD) & 0.0891991 & 0.0241885 & 3.68767 & $2.26 \mathrm{E}-04$ \\
\hline Alpha & 0.217963 & 0.0195015 & 11.1767 & $6.44 \mathrm{E}-14$ \\
\hline $\begin{array}{l}\text { Number of observations } \\
\text { Log likelihood function } \\
\text { Restricted log likelihood = } \\
\text { Chi-squared = } \\
\text { Response variable is the Natural Log }\end{array}$ & $\begin{array}{l}524 \\
-1961.263 \\
-2633.962 \\
654.824[.0 \\
\text { Number of }\end{array}$ & & & \\
\hline
\end{tabular}


Table D-9: Injury Crash Model for Rural Principal Arterials

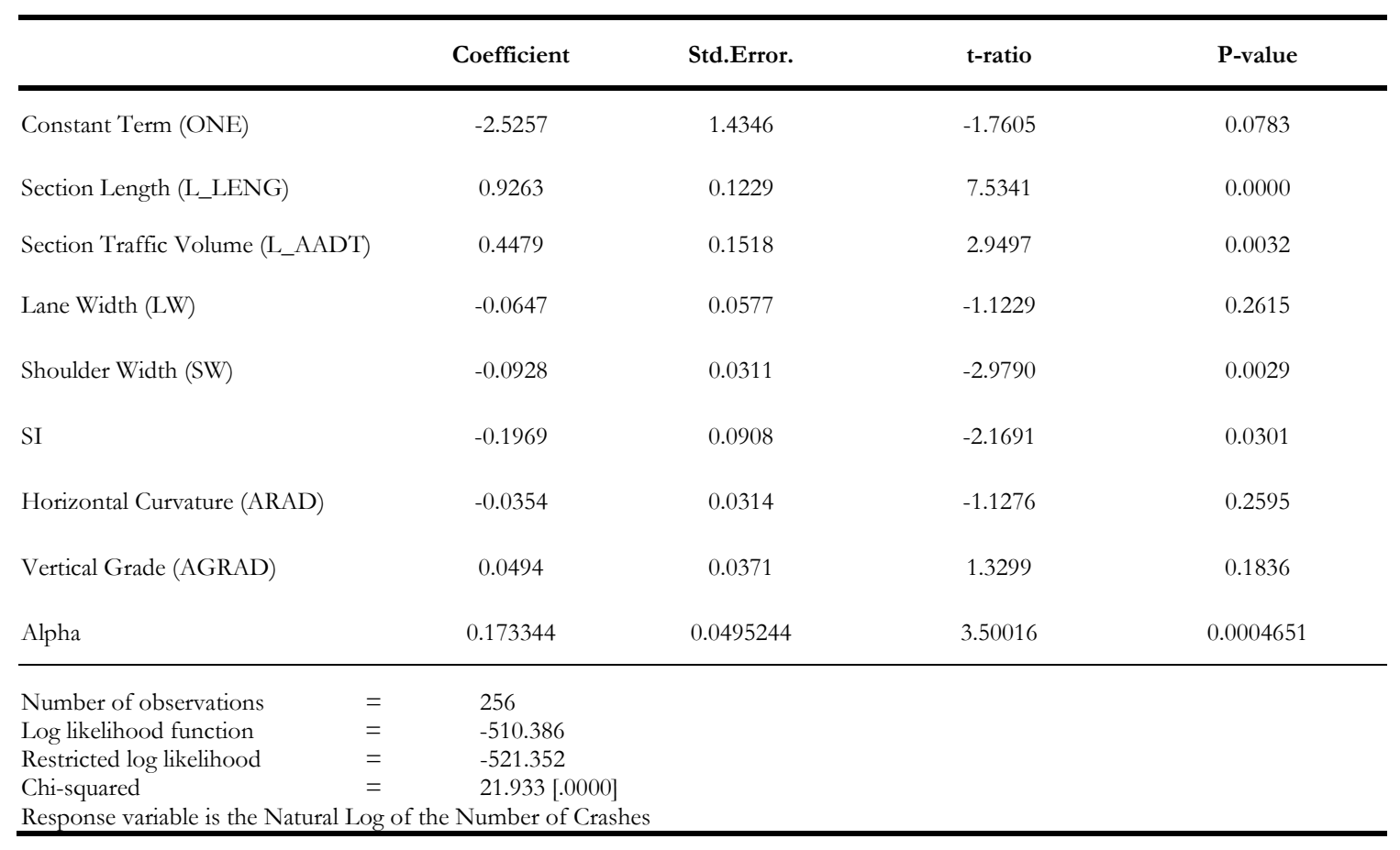

Table D-10: PDO Crash Model for Rural Principal Arterials

\begin{tabular}{|c|c|c|c|c|}
\hline & Coefficient & Std.Error. & t-ratio & P-value \\
\hline Constant Term (ONE) & 0.8921 & 1.2246 & 0.7285 & 0.4663 \\
\hline Section Length (L_LENG) & 0.7097 & 0.0681 & 10.4180 & 0.0000 \\
\hline Section Traffic Volume (L_AADT) & 0.2409 & 0.1204 & 2.0013 & 0.0454 \\
\hline Lane Width (LW) & -0.1128 & 0.0596 & -1.8914 & 0.0586 \\
\hline Shoulder Width (SW) & -0.0676 & 0.0183 & -3.6990 & 0.0002 \\
\hline SI & -0.0624 & 0.0612 & -1.0194 & 0.3080 \\
\hline Horizontal Curvature (ARAD) & -0.0553 & 0.0272 & -2.0316 & 0.0422 \\
\hline Vertical Grade (AGRAD) & 0.0646 & 0.0280 & 2.3110 & 0.0208 \\
\hline Alpha & 0.199856 & 0.0300089 & 6.65988 & $2.76 \mathrm{E}-11$ \\
\hline $\begin{array}{l}\text { Number of observations } \\
\text { Log likelihood function } \\
\text { Restricted log likelihood } \\
\text { Chi-squared } \\
\text { Response variable is the Natural Log }\end{array}$ & $\begin{array}{l}256 \\
-771.641 \\
-864.080 \\
184.879 \\
\text { Number of } \\
\end{array}$ & & & \\
\hline
\end{tabular}


Table D-11: Fatal+Injury Crash Model for Rural Principal Arterials

\begin{tabular}{|c|c|c|c|c|}
\hline & Coefficient & Std.Error. & t-ratio & P-value \\
\hline Constant Term (ONE) & -2.2394 & 1.4733 & -1.5200 & 0.1285 \\
\hline Section Length (L_LENG) & 0.9231 & 0.1251 & 7.3783 & 0.0000 \\
\hline Section Traffic Volume (L_AADT) & 0.4499 & 0.1529 & 2.9422 & 0.0033 \\
\hline Lane Width (LW) & -0.0837 & 0.0596 & -1.4037 & 0.1604 \\
\hline Shoulder Width (SW) & -0.0943 & 0.0304 & -3.0987 & 0.0019 \\
\hline SI & -0.1962 & 0.0936 & -2.0955 & 0.0361 \\
\hline Horizontal Curvature (ARAD) & -0.0364 & 0.0307 & -1.1865 & 0.2354 \\
\hline Vertical Grade (AGRAD) & 0.0489 & 0.0369 & 1.3236 & 0.1856 \\
\hline Alpha & 0.193443 & 0.0490488 & 3.94389 & $8.02 \mathrm{E}-05$ \\
\hline $\begin{array}{lr}\text { Number of observations } & = \\
\text { Log likelihood function } & = \\
\text { Restricted log likelihood } & = \\
\text { Chi-squared } & = \\
\text { Response variable is the Natural Log }\end{array}$ & $\begin{array}{l}256 \\
-523.039 \\
-537.623 \\
29.168[.0000] \\
\text { Number of Cras }\end{array}$ & & & \\
\hline
\end{tabular}

Table D-12: “Total Crashes” Model for Rural Principal Arterials

\begin{tabular}{|c|c|c|c|c|}
\hline & Coefficient & Std.Error. & t-ratio & P-value \\
\hline Constant Term (ONE) & 0.6071 & 1.2046 & 0.5040 & 0.6143 \\
\hline Section Length (L_LENG) & 0.7537 & 0.0660 & 11.4189 & 0.0000 \\
\hline Section Traffic Volume (L_AADT) & 0.3014 & 0.1184 & 2.5457 & 0.0109 \\
\hline Lane Width (LW) & -0.1079 & 0.0559 & -1.9316 & 0.0534 \\
\hline Shoulder Width (SW) & -0.0734 & 0.0188 & -3.9120 & 0.0001 \\
\hline SI & -0.0814 & 0.0595 & -1.3681 & 0.1713 \\
\hline Horizontal Curvature (ARAD) & -0.0540 & 0.0259 & -2.0814 & 0.0374 \\
\hline Vertical Grade (AGRAD) & 0.0574 & 0.0283 & 2.0277 & 0.0426 \\
\hline Alpha & 0.201991 & 0.0266358 & 7.58345 & $6.44 \mathrm{E}-14$ \\
\hline $\begin{array}{l}\text { Number of observations } \\
\text { Log likelihood function = } \\
\text { Restricted log likelihood = } \\
\text { Chi-squared = } \\
\text { Response variable is the Natural Log }\end{array}$ & $\begin{array}{l}256 \\
-829.307 \\
-963.728 \\
268.842[.00 \\
\text { umber of } \mathrm{Cr}\end{array}$ & & & \\
\hline
\end{tabular}


Table D-13: Model for County Road Crashes Excluding Shoulder Width Variable

\begin{tabular}{|c|c|c|c|c|}
\hline Variable & Coefficient & Std.Error. & t-ratio & P-value \\
\hline Constant Term (ONE) & -1.45459 & 0.9519 & -1.53 & 0.130 \\
\hline Log Section Length (LOG_L_LENG) & -0.336618 & 0.2413 & -1.39 & 0.167 \\
\hline Log Section Traffic Volume (log AADT) & 0.825750 & 0.1733 & 4.76 & 0.000 \\
\hline Lane Width (LW) & -0.358197 & 0.1301 & -2.75 & 0.007 \\
\hline 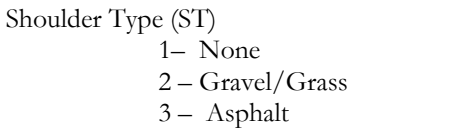 & -0.412005 & 0.4081 & -1.01 & 0.315 \\
\hline Driveway Density (DD) & 0.0191902 & 0.01803 & 1.06 & 0.290 \\
\hline $\begin{array}{l}\text { Speed Limit Posted (SPL) } \\
\qquad \begin{array}{l}1-\text { None Posted } \\
2-\text { Speed Limit Posted }\end{array}\end{array}$ & 0.571718 & 0.3602 & 1.59 & 0.116 \\
\hline $\begin{array}{l}\text { Horizontal Curve Density (HCD) } \\
\text { Road Surface Type (RST) } \\
\qquad \begin{array}{c}1-\text { Gravel } \\
2-\text { ChipSeal/SandSeal } \\
3 \text { - Asphalt }\end{array}\end{array}$ & -0.448448 & 0.1346 & -1.47 & 0.145 \\
\hline Alpha & -0.929138 & 0.4195 & -2.21 & 0.029 \\
\hline $\begin{array}{lc}\text { Number of observations } & = \\
\text { Log likelihood function } & = \\
\text { baseline log-likelihood } & = \\
\text { Chi-squared } & = \\
\text { Response variable is the Natural Log of th }\end{array}$ & $\begin{array}{l}98 \\
-163.317 \\
-207.7305 \\
88.827[0.0000] \\
\text { Jumber of Crash }\end{array}$ & & & \\
\hline
\end{tabular}

Table D-14: Model for County Road Crashes Including Shoulder Width Variable

\begin{tabular}{|c|c|c|c|c|}
\hline Variable & Coefficient & Std.Error. & t-ratio & P-value \\
\hline Constant Term (ONE) & -5.00725 & 2.388 & -2.10 & 0.081 \\
\hline Log Section Length (LOG_L_LENG) & -0.112947 & 0.3161 & -0.357 & 0.733 \\
\hline Log Section Traffic Volume (log AADT) & 0.833491 & 0.2657 & 3.14 & 0.020 \\
\hline Shoulder Width (SW) & -0.181543 & 0.08198 & -2.21 & 0.069 \\
\hline $\begin{array}{lc}\text { Number of observations } & = \\
\text { Log likelihood function } & = \\
\text { baseline log-likelihood } & = \\
\text { Chi-squared } & = \\
\text { Response variable is the Natural Log of th }\end{array}$ & $\begin{array}{l}10 \\
-16.3989444 \\
-28.88138 \\
24.965[0.0000] \\
\text { Uumber of Crashe }\end{array}$ & & & \\
\hline
\end{tabular}




\section{APPENDIX E PRIORITY LISTING OF SAFETY PROJECTS}

Table E-1 Priority Ranking of Selected Safety Improvement Project, Scenario 2

Budgetary Limit: None

Basis of Selection: Sections that are both Deficient and Hazardous

\begin{tabular}{|c|c|c|c|c|c|c|c|}
\hline Rank & Section ID & FC & County & Location & Safety Project & Capital & Length (mi) \\
\hline 1 & $41-S-144-0-01$ & RMC & Johnson & $0.00 \mathrm{mi}-0.20 \mathrm{mi}$ & Install guardrail & $\$ 36,880$ & 0.20 \\
\hline 2 & 87-S-261-0-01 & RMC & Warrick & $0.00 \mathrm{mi}-3.76 \mathrm{mi}$ & Install guardrail & $\$ 693,348$ & 3.76 \\
\hline 3 & 45-U-231-0-01 & $\mathrm{RMC}$ & Lake & $0.00 \mathrm{mi}-2.12 \mathrm{mi}$ & Widen Shoulder by $4 \mathrm{ft}$ & $\$ 518,488$ & 2.12 \\
\hline 4 & 87-S-066-0-01 & RPA & Warrick & $0.00 \mathrm{mi}-6.85 \mathrm{mi}$ & Install guardrail & $\$ 1,263,147$ & 6.85 \\
\hline 5 & 57-U-033-0-01 & RPA & Noble & $16.72 \mathrm{mi}-17.31 \mathrm{mi}$ & Widen Shoulder by $4 \mathrm{ft}$ & $\$ 144,296$ & 0.59 \\
\hline 6 & 29-U-421-0-01 & RMA & Hamilton & $0.00 \mathrm{mi}-1.77 \mathrm{mi}$ & $\begin{array}{l}\text { Widen Roadway lane by } 1 \\
\mathrm{ft} \& \text { Widen Shoulder by } 2 \\
\mathrm{ft}\end{array}$ & $\$ 57,778$ & 0.33 \\
\hline 7 & 86-U-041-0-01 & RMA & Warren & $0.00 \mathrm{mi}-0.62 \mathrm{mi}$ & Install paved shoulder & $\$ 98,303$ & 0.29 \\
\hline 8 & 44-S-009-0-01 & RMA & Lagrange & $13.57 \mathrm{mi}-17.18 \mathrm{mi}$ & Install paved shoulder & $\$ 142,370$ & 0.42 \\
\hline 9 & 64-U-006-0-01 & RMA & Porter & $5.22 \mathrm{mi}-10.24 \mathrm{mi}$ & Install guardrail & $\$ 925,693$ & 5.02 \\
\hline 10 & 03-S-007-0-01 & RPA & Bartholomew & $4.38 \mathrm{mi}-5.14 \mathrm{mi}$ & Widen Shoulder by $4 \mathrm{ft}$ & $\$ 173,645$ & 0.71 \\
\hline 11 & $36-U-050-0-01$ & RPA & Jackson & $8.54 \mathrm{mi}-12.27 \mathrm{mi}$ & Widen Shoulder by $4 \mathrm{ft}$ & $\$ 912,246$ & 3.73 \\
\hline 12 & 79-S-043-0-01 & RPA & Tippecanoe & $3.30 \mathrm{mi}-10.08 \mathrm{mi}$ & Install guardrail & $\$ 1,250,239$ & 6.78 \\
\hline 13 & $35-U-224-0-01$ & RMA & Huntington & $7.34 \mathrm{mi}-8.69 \mathrm{mi}$ & Widen Shoulder by $4 \mathrm{ft}$ & $\$ 330,170$ & 1.35 \\
\hline 14 & 64-S-002-0-01 & RMA & Porter & $13.76 \mathrm{mi}-18.99 \mathrm{mi}$ & Install guardrail & $\$ 964,417$ & 5.23 \\
\hline 15 & 91-U-024-0-01 & RPA & White & $16.29 \mathrm{mi}-22.76 \mathrm{mi}$ & $\begin{array}{l}\text { Widen Shoulder by } 2 \mathrm{ft} \& \\
\text { Install guardrail }\end{array}$ & $\$ 1,984,252$ & 6.47 \\
\hline 16 & $10-S-062-0-01$ & RMA & Clark & $7.45 \mathrm{mi}-16.12 \mathrm{mi}$ & $\begin{array}{l}\text { Widen Shoulder by } 2 \mathrm{ft} \& \\
\text { Install guardrail }\end{array}$ & $\$ 2,658,959$ & 8.67 \\
\hline 17 & 66-U-035-0-01 & RMA & Pulaski & $13.47 \mathrm{mi}-19.20 \mathrm{mi}$ & $\begin{array}{l}\text { Widen Roadway lane by } 1 \\
\mathrm{ft} \text { in both directions }\end{array}$ & $\$ 302,544$ & 5.73 \\
\hline 18 & 79-S-025-0-01 & RPA & Tippecanoe & $27.55 \mathrm{mi}-36.80 \mathrm{mi}$ & $\begin{array}{l}\text { Widen Shoulder by } 2 \mathrm{ft} \& \\
\text { Install guardrail }\end{array}$ & $\$ 2,836,836$ & 9.25 \\
\hline 19 & 17-S-427-0-01 & $\mathrm{RMC}$ & Dekalb & $0.00 \mathrm{mi}-0.62 \mathrm{mi}$ & Install paved shoulder & $\$ 132,201$ & 0.39 \\
\hline 20 & 92-U-033-0-01 & RPA & Whitley & $0.00 \mathrm{mi}-3.20 \mathrm{mi}$ & Install paved shoulder & $\$ 322,027$ & 0.95 \\
\hline 21 & 02-S-037-0-01 & RMC & Allen & $12.84 \mathrm{mi}-14.27 \mathrm{mi}$ & $\begin{array}{l}\text { Widen Roadway lane by } 1 \\
\mathrm{ft} \& \text { Widen Shoulder by } 2 \\
\mathrm{ft}\end{array}$ & $\$ 250,370$ & 1.43 \\
\hline 22 & 32-S-267-0-01 & RMA & Hendricks & $8.68 \mathrm{mi}-14.29 \mathrm{mi}$ & Widen Shoulder by $4 \mathrm{ft}$ & $\$ 1,372,038$ & 5.61 \\
\hline 23 & 79-U-231-0-01 & RPA & Tippecanoe & $5.96 \mathrm{mi}-12.54 \mathrm{mi}$ & Install guardrail & $\$ 1,213,359$ & 6.58 \\
\hline 24 & 78-S-129-0-01 & $\mathrm{RMC}$ & Switzerland & $0.00 \mathrm{mi}-0.94 \mathrm{mi}$ & Do Nothing & $\$ 0$ & 0.00 \\
\hline 25 & $64-S-002-0-01$ & $\mathrm{RMC}$ & Porter & $18.99 \mathrm{mi}-25.56 \mathrm{mi}$ & Install guardrail & $\$ 1,211,515$ & 6.57 \\
\hline 26 & $55-S-044-0-01$ & $\mathrm{RMC}$ & Morgan & $0.00 \mathrm{mi}-2.08 \mathrm{mi}$ & Install guardrail & $\$ 383,554$ & 2.08 \\
\hline 27 & 42-S-067-0-01 & RMA & Knox & $0.00 \mathrm{mi}-1.63 \mathrm{mi}$ & Widen Shoulder by $4 \mathrm{ft}$ & $\$ 398,649$ & 1.63 \\
\hline 28 & 73-U-052-0-01 & RMA & Shelby & $0.00 \mathrm{mi}-1.30 \mathrm{mi}$ & Widen Shoulder by $4 \mathrm{ft}$ & $\$ 317,941$ & 1.30 \\
\hline
\end{tabular}


Table E-1 Priority Ranking of Selected Safety Improvement Project, Scenario 2 (continued)

\begin{tabular}{|c|c|c|c|c|c|c|c|}
\hline Rank & Section ID & FC & County & Location & Safety Project & Capital & Length (mi) \\
\hline 29 & 13-S-066-0-01 & $\mathrm{RMC}$ & Crawford & $16.00 \mathrm{mi}-18.13 \mathrm{mi}$ & Install paved shoulder & $\$ 722,019$ & 2.13 \\
\hline 30 & 31-S-011-0-01 & RMC & Harrison & $17.73 \mathrm{mi}-23.96 \mathrm{mi}$ & $\begin{array}{l}\text { Widen Roadway lane by } 1 \\
\text { ft \& Install guardrail }\end{array}$ & $\$ 1,477,762$ & 6.23 \\
\hline 31 & 19-S-056-0-01 & RMA & Dubois & $22.85 \mathrm{mi}-29.06 \mathrm{mi}$ & Install guardrail & $\$ 1,145,130$ & 6.21 \\
\hline 32 & 09-U-024-0-01 & RPA & Cass & $6.05 \mathrm{mi}-8.50 \mathrm{mi}$ & Widen Shoulder by $4 \mathrm{ft}$ & $\$ 599,197$ & 2.45 \\
\hline 33 & 12-S-038-0-01 & RMC & Clinton & $0.00 \mathrm{mi}-2.23 \mathrm{mi}$ & Widen Shoulder by $4 \mathrm{ft}$ & $\$ 545,391$ & 2.23 \\
\hline 34 & 29-S-238-0-01 & $\mathrm{RMC}$ & Hamilton & $3.56 \mathrm{mi}-9.60 \mathrm{mi}$ & Install guardrail & $\$ 1,113,782$ & 6.04 \\
\hline 35 & $10-S-160-0-01$ & $\mathrm{RMC}$ & Clark & $4.97 \mathrm{mi}-13.06 \mathrm{mi}$ & Install guardrail & $\$ 1,491,804$ & 8.09 \\
\hline 36 & 78-S-056-0-01 & RMC & Switzerland & $6.31 \mathrm{mi}-17.49 \mathrm{mi}$ & Install guardrail & $\$ 2,061,603$ & 11.18 \\
\hline 37 & 58-S-056-0-01 & RMA & Ohio & $2.25 \mathrm{mi}-15.61 \mathrm{mi}$ & Install guardrail & $\$ 2,463,597$ & 13.36 \\
\hline 38 & 92-S-009-0-01 & RMA & Whitley & $9.96 \mathrm{mi}-12.94 \mathrm{mi}$ & $\begin{array}{l}\text { Horizontal curve re- } \\
\text { alignment }\end{array}$ & $\$ 77,250$ & 0.10 \\
\hline 39 & 61-U-036-0-01 & RMA & Parke & $0.00 \mathrm{mi}-1.53 \mathrm{mi}$ & Widen Shoulder by $4 \mathrm{ft}$ & $\$ 374,192$ & 1.53 \\
\hline 40 & 43-S-015-0-01 & RMA & Kosciusko & $0.00 \mathrm{mi}-4.08 \mathrm{mi}$ & $\begin{array}{l}\text { Widen Shoulder by } 2 \mathrm{ft} \& \\
\text { Install guardrail }\end{array}$ & $\$ 1,251,275$ & 4.08 \\
\hline 41 & 55-S-039-0-01 & RMC & Morgan & $7.04 \mathrm{mi}-11.07 \mathrm{mi}$ & $\begin{array}{l}\text { Widen Roadway lane by } 1 \\
\mathrm{ft} \& \text { Install guardrail }\end{array}$ & $\$ 955,920$ & 4.03 \\
\hline 42 & 88-S-135-0-01 & RMA & Washington & $0.00 \mathrm{mi}-4.16 \mathrm{mi}$ & Install guardrail & $\$ 767,108$ & 4.16 \\
\hline 43 & 40-U-050-0-01 & RPA & Jennings & $0.00 \mathrm{mi}-6.87 \mathrm{mi}$ & Install guardrail & $\$ 1,266,835$ & 6.87 \\
\hline 44 & 09-U-024-0-01 & RPA & Cass & $0.00 \mathrm{mi}-6.05 \mathrm{mi}$ & Install guardrail & $\$ 1,115,626$ & 6.05 \\
\hline 45 & 07-S-046-0-01 & RPA & Brown & $0.00 \mathrm{mi}-18.35 \mathrm{mi}$ & Install guardrail & $\$ 3,383,758$ & 18.35 \\
\hline 46 & 75-S-008-0-01 & RMC & Starke & $10.75 \mathrm{mi}-16.51 \mathrm{mi}$ & $\begin{array}{l}\text { Install paved shoulder \& } \\
\text { Install guardrail }\end{array}$ & $\$ 3,014,652$ & 5.76 \\
\hline 47 & 61-U-041-0-01 & RMA & Parke & $7.35 \mathrm{mi}-10.82 \mathrm{mi}$ & Install guardrail & $\$ 639,871$ & 3.47 \\
\hline 48 & 24-U-052-0-01 & RPA & Franklin & $25.69 \mathrm{mi}-30.00 \mathrm{mi}$ & Install guardrail & $\$ 794,768$ & 4.31 \\
\hline 49 & 15-S-048-0-01 & RMC & Dearborn & $0.00 \mathrm{mi}-10.38 \mathrm{mi}$ & $\begin{array}{l}\text { Widen Roadway lane by } 1 \\
\mathrm{ft} \& \text { Install guardrail }\end{array}$ & $\$ 2,462,146$ & 10.38 \\
\hline 50 & 63-S-064-0-01 & RMA & Pike & $0.00 \mathrm{mi}-4.21 \mathrm{mi}$ & Widen Shoulder by $4 \mathrm{ft}$ & $\$ 1,029,640$ & 4.21 \\
\hline 51 & 15-U-052-0-01 & RPA & Dearborn & $0.00 \mathrm{mi}-3.04 \mathrm{mi}$ & Widen Shoulder by $4 \mathrm{ft}$ & $\$ 743,493$ & 3.04 \\
\hline 52 & 09-S-025-0-01 & RPA & Cass & $0.00 \mathrm{mi}-5.72 \mathrm{mi}$ & Install guardrail & $\$ 1,054,774$ & 5.72 \\
\hline 53 & 81-S-044-0-01 & RMA & Union & $0.00 \mathrm{mi}-5.17 \mathrm{mi}$ & Widen Shoulder by $4 \mathrm{ft}$ & $\$ 1,100,565$ & 4.50 \\
\hline 54 & 89-U-027-0-01 & RPA & Wayne & $4.61 \mathrm{mi}-13.14 \mathrm{mi}$ & Do Nothing & $\$ 0$ & 0.00 \\
\hline 55 & 09-U-024-0-01 & RPA & Cass & $8.50 \mathrm{mi}-11.34 \mathrm{mi}$ & Widen Shoulder by $4 \mathrm{ft}$ & $\$ 484,249$ & 1.98 \\
\hline 56 & 44-U-020-0-01 & RMA & Lagrange & $0.00 \mathrm{mi}-6.00 \mathrm{mi}$ & $\begin{array}{l}\text { Widen Roadway lane by } 1 \\
\mathrm{ft} \text { in both directions }\end{array}$ & $\$ 212,784$ & 4.03 \\
\hline 57 & 57-U-033-0-01 & RPA & Noble & $11.37 \mathrm{mi}-16.72 \mathrm{mi}$ & Widen Shoulder by $4 \mathrm{ft}$ & $\$ 1,308,450$ & 5.35 \\
\hline 58 & 35-U-024-0-01 & RPA & Huntington & $0.00 \mathrm{mi}-4.56 \mathrm{mi}$ & $\begin{array}{l}\text { Widen Roadway lane by } 1 \\
\mathrm{ft} \text { in both directions }\end{array}$ & $\$ 240,768$ & 4.56 \\
\hline \multicolumn{6}{|c|}{ Total } & $\$ 54,793,673$ & 254.44 \\
\hline
\end{tabular}


APPENDIX F: RELATIONSHIP BETWEEN CRASH FREQUENCY AND TRAFFIC VOLUME

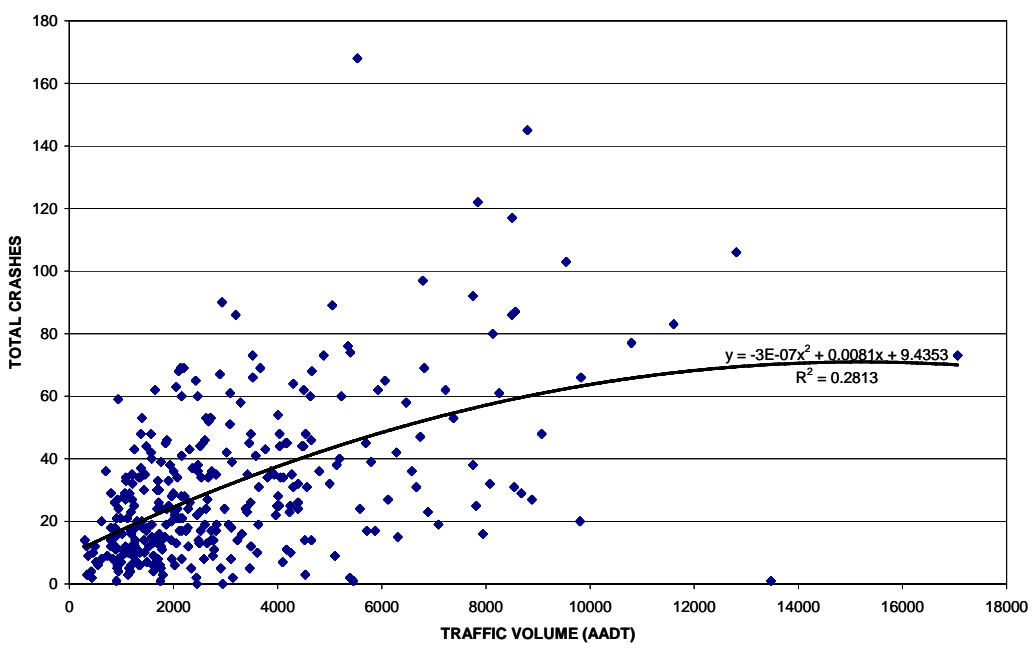

(a) Rural

Major Collectors

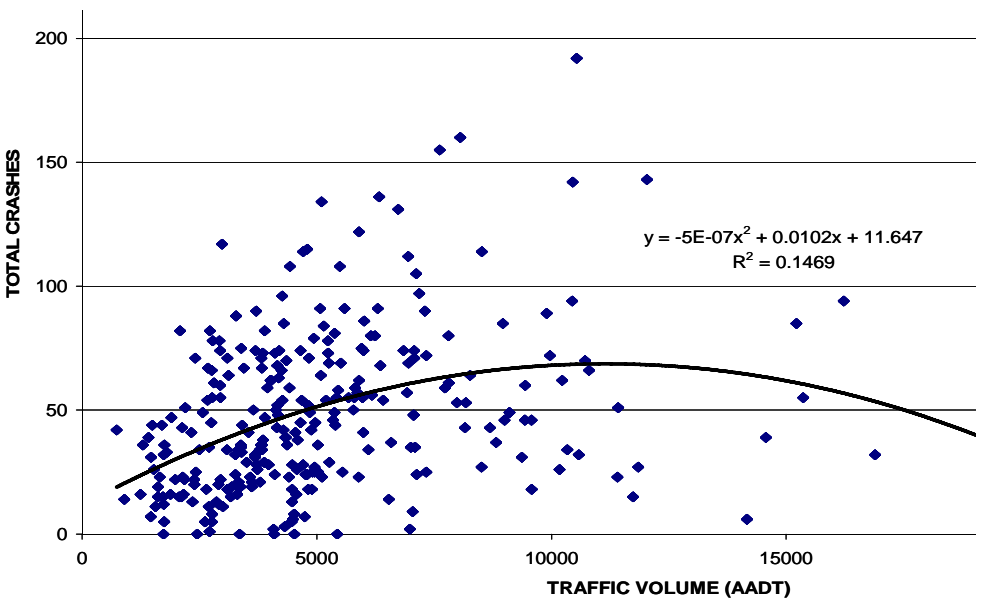

(b) Rural

Minor Arterials

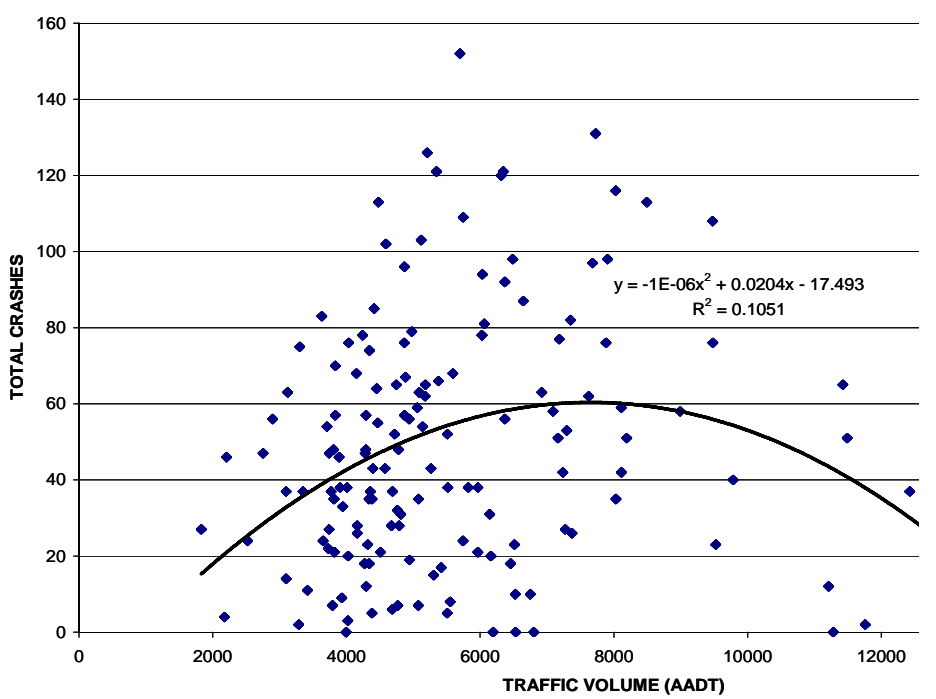

(c) Rural

Principal Arterials 